\title{
GLOBAL STRONG SOLUTIONS OF THE VLASOV-POISSON-BOLTZMANN SYSTEM IN BOUNDED DOMAINS
}

\author{
YUNBAI CAO, CHANWOO KIM, AND DONGHYUN LEE
}

\begin{abstract}
When dilute charged particles are confined in a bounded domain, boundary effects are crucial in the global dynamics. We construct a unique global-in-time solution to the Vlasov-Poisson-Boltzmann system in convex domains with the diffuse boundary condition. The construction is based on an $L^{2}-L^{\infty}$ framework with a novel nonlinear-normed energy estimate of a distribution function in weighted $W^{1, p}$-spaces and a $C^{2, \delta}$-estimate of the self-consistent electric potential. Moreover we prove an exponential convergence of the distribution function toward the global Maxwellian.
\end{abstract}

\section{CONTEnts}

1. Introduction

1.1. A New Kinetic Weight

1.2. Main Results

1.3. Nonlinear-Normed Energy Estimates and an Interpolation

1.4. Desingularization via Mixing in Velocity

1.5. $L^{\infty}$-Estimate

1.6. A Priori $L_{x}^{3} L_{v}^{1+}$-Estimate of $\nabla_{v} f$ and $L^{1+}$-Stability

2. Preliminary and In-Flow Problems

3. Desingularization via Mixing in the Velocity space

4. Nonlinear-Normed Energy Estimates in Weighted $W^{1, p}$

5. $\quad L_{x}^{3} L_{v}^{1+}$-Estimate of $\nabla_{v} f$ and $L^{1+}$-Stability

6. Local Existence

7. $L^{2}$ coercivity

8. Global Existence and Exponential decay

Appendix A. Auxiliary Results and Proofs

References

\section{INTRODUCTION}

The object of kinetic theory is the modeling of many particles whose behavior is encoded in a distribution function on the phase space, which is denoted by $F(t, x, v)$ for $(t, x, v) \in[0, \infty) \times \Omega \times \mathbb{R}^{3}$. Here $\Omega$ denotes an open subset of $\mathbb{R}^{3}$. Dynamics and collision processes of dilute charged particles subjected to an field $E$ can be modeled by the Vlasov-Boltzmann equation:

$$
\partial_{t} F+v \cdot \nabla_{x} F+E \cdot \nabla_{v} F=Q(F, F) .
$$

The collision operator measures the change rate in binary hard sphere collisions and takes the form of

$$
\begin{aligned}
Q\left(F_{1}, F_{2}\right)(v) & :=Q_{\text {gain }}\left(F_{1}, F_{2}\right)-Q_{\text {loss }}\left(F_{1}, F_{2}\right) \\
& :=\int_{\mathbb{R}^{3}} \int_{\mathbb{S}^{2}}|(v-u) \cdot \omega|\left[F_{1}\left(u^{\prime}\right) F_{2}\left(v^{\prime}\right)-F_{1}(u) F_{2}(v)\right] \mathrm{d} \omega \mathrm{d} u,
\end{aligned}
$$

where $u^{\prime}=u-[(u-v) \cdot \omega] \omega$ and $v^{\prime}=v+[(u-v) \cdot \omega] \omega$. The collision operator enjoys a collision invariance: for any measurable $G$,

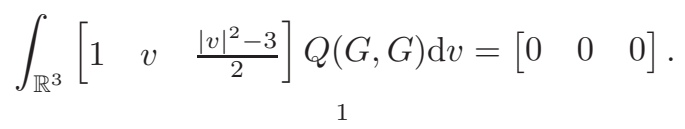


It is well-known that a global Maxwellian $\mu$ satisfies $Q(\mu, \mu)=0$, where

$$
\mu(v):=\frac{1}{(2 \pi)^{3 / 2}} \exp \left(-\frac{|v|^{2}}{2}\right) .
$$

Due to its importance of the Boltzmann equation in theories and their applications, there have been explosive research results in analytic study of the equation. The nonlinear energy method has led to solutions of many open problems $(13,14)$ including global classical solutions and their asymptotic stability of Boltzmann equation coupled with either the Poisson equation or the Maxwell system for electromagnetism when the initial data are close to the Maxwellian $\mu$. For large-amplitude solutions, renormalized solutions have been studied extensively from the end of 80's (see [7, 34] and references therein). An asymptotic stability of some large-amplitude solutions is established in [10, 12, provided certain a priori strong Sobolev estimates can be verified. Such high regularity insures an $L^{\infty}$-control of solutions which is crucial to handle the quadratic nonlinearity. It should be noted that all of these results deal with idealized periodic domains or whole space, in which the solutions can remain bounded in high Sobolev spaces.

In many important physical applications, e.g. semiconductor and tokamak, the charged dilute gas is confined within a container, and its interaction with the boundary often plays a crucial role in global dynamics. The interaction of the gas with a boundary is described by a suitable boundary conditions [32]. In this paper we consider one of the physical conditions, a so-called diffuse boundary condition:

$$
F(t, x, v)=c_{\mu} \mu(v) \int_{n(x) \cdot u>0} F(t, x, u)\{n(x) \cdot u\} \mathrm{d} u \quad \text { for }(x, v) \in \gamma_{-} .
$$

Here, an outgoing set is defined as

$$
\gamma_{-}:=\left\{(x, v) \in \partial \Omega \times \mathbb{R}^{3}: n(x) \cdot v<0\right\}
$$

and $n(x)$ is the outward unit normal at a boundary point $x$. A number $c_{\mu}$ is chosen to be $\sqrt{2 \pi}$ so that $c_{\mu} \int_{n(x) \cdot u>0} \mu(u)\{n(x) \cdot u\} \mathrm{d} u=1$ with $\mu$ in (1.4). Due to this normalization, the distribution of (1.5) enjoys a null flux condition at the boundary:

$$
\int_{\mathbb{R}^{3}} F(t, x, v)\{n(x) \cdot v\} \mathrm{d} v=0 \quad \text { for } x \in \partial \Omega .
$$

In general, high regularity may not be expected for solutions of the Boltzmann equation in physical bounded domains. Such a drastic boundary effect has been demonstrated recently by the second author and his collaborators as the formation and propagation of discontinuity in non-convex domains in [25, 9, and a non-existence of some second order derivative at the boundary even in convex domains [15]. Evidently these results show that smooth solutions are generally unavailable to the boundary problems (cf. [10]).

In order to overcome such critical difficulty, an $L^{2}-L^{\infty}$ framework has been developed in [17] to study the Boltzmann equation with various boundary conditions. The core of the method lays in a direct approach (without taking derivatives) to achieve a pointwise bound using trajectory of the transport operator, which leads substantial development in various directions ([8, 9, 15, 16, 27, 28, 29, 26, 35, 18, 30, 29, 5]). Among others we briefly point out some relevant works to the Vlasov-Boltzmann equation (1.1) in bounded domains. In [27, the second and the third authors established the global well-posedness and asymptotic stability of the Boltzmann equation near the global Maxwellian for the specular reflection boundary condition with or without given small external fields. In [8], the second author and other collaborators studied diffusive hydrodynamic limit of Boltzmann to Navier-Stokes-Fourier system in both steady and unsteady cases in the presence of given small external fields.

However, in the study of charged particles generating self-consistent fields, many fundamental boundary problems of the kinetic models are still widely open. This category of problems is important in the application to plasma or galaxy. One of the major difficulties is that trajectories are curved and behave in a very complicated way when they hit the boundary.

The field $E$, that we are interested in, is associated with an electrostatic potential $\phi$ as

$$
E(t, x):=-\nabla \phi(t, x)
$$

where the potential is determined by the Poisson equation:

$$
-\Delta \phi(t, x)=\int_{\mathbb{R}^{3}} F(t, x, v) \mathrm{d} v-\rho_{0} \text { in } \Omega,
$$


with the zero Neumann boundary condition

$$
\frac{\partial \phi}{\partial n}=0 \text { on } \partial \Omega
$$

Here a background density $\rho_{0}$ in (1.9) is a constant number.

The coupled system of (1.1) with (1.8) and (1.9) is a so-called Vlasov-Poisson-Boltzmann system (VPB), which describes the dynamics of electrons in the absence of a magnetic field. This model has been considered as a fundamental collisional plasma model and has attracted much attention (see [6, 13, 18, 22, 33, and the references therein).

From (1.3) and (1.7), a strong solution of VPB with the diffuse BC (1.5) preserves total mass:

$$
\iint_{\Omega \times \mathbb{R}^{3}} F(t, x, v) \mathrm{d} v \mathrm{~d} x \equiv \iint_{\Omega \times \mathbb{R}^{3}} F(0, x, v) \mathrm{d} v \mathrm{~d} x \text { for all } t \geq 0 .
$$

We set, without loss of generality,

$$
\rho_{0}=\frac{1}{|\Omega|} \iint_{\Omega \times \mathbb{R}^{3}} F(0, x, v) \mathrm{d} v \mathrm{~d} x . \quad \text { (a neutral condition) }
$$

Then $\int_{\Omega}\left\{\int_{\mathbb{R}^{3}} F(t, x, v) \mathrm{d} v-\rho_{0}\right\} \mathrm{d} x=0$ for all $t>0$ from (1.11). This zero-mean condition guarantees a solvability of the Poisson equation (1.9) with the Neumann boundary condition (1.10).

Without loss of generality, by some rescaling, we assume that

$$
\iint_{\Omega \times \mathbb{R}^{3}} \mu \mathrm{d} v \mathrm{~d} x=\iint_{\Omega \times \mathbb{R}^{3}} F(0, x, v) \mathrm{d} v \mathrm{~d} x .
$$

We consider a perturbation around $\mu$ :

$$
F(t, x, v)=\mu+\sqrt{\mu} f(t, x, v) .
$$

The corresponding problem of (1.14) is given by

$$
\partial_{t} f+v \cdot \nabla_{x} f-\nabla_{x} \phi_{f} \cdot \nabla_{v} f+\frac{v}{2} \cdot \nabla_{x} \phi_{f} f+L f=\Gamma(f, f)-v \cdot \nabla_{x} \phi_{f} \sqrt{\mu},
$$

with $f(0, x, v)=f_{0}(x, v)$ and

$$
-\Delta \phi_{f}(t, x)=\int_{\mathbb{R}^{3}} f(t, x, v) \sqrt{\mu(v)} \mathrm{d} v \text { in } \Omega, \quad \frac{\partial \phi_{f}}{\partial n}=0 \quad \text { on } \partial \Omega,
$$

and

$$
f(t, x, v)=c_{\mu} \sqrt{\mu} \int_{n(x) \cdot u>0} f(t, x, u) \sqrt{\mu(u)}\{n(x) \cdot u\} \mathrm{d} u \quad \text { for }(x, v) \in \gamma_{-} .
$$

We note that from (1.11) and (1.13)

$$
\int_{\Omega} \int_{\mathbb{R}^{3}} f(t, x, v) \sqrt{\mu(v)} \mathrm{d} v \mathrm{~d} x=0 \text { for all } t \geq 0 .
$$

The standard notions of $L, \nu, K$, and $\Gamma$ are defined in (2.1)-(2.5).

1.1. A New Kinetic Weight. An intrinsic feature of the transport equation in bounded domains is the singular behavior of its derivatives. Let us consider a solution of the Vlasov-Poisson equation

$$
\partial_{t} f+v \cdot \nabla_{x} f-\nabla_{x} \phi_{f} \cdot \nabla_{v} f=0
$$

where $\phi_{f}(t, x)$ satisfies (1.16). Assume that the boundary condition is determined by some given function $g$ as

$$
f(t, x, v)=g(t, x, v) \quad \text { for }(x, v) \in \gamma_{-} .
$$

Throughout this paper we extend $\phi_{f}$ for a negative time as

$$
\phi_{f}(t, x):=e^{-|t|} \phi_{f_{0}}(x) \text { in (1.16) for }-\infty<t<0 .
$$

Note that, for all $t \in \mathbb{R}, \phi_{f}(t, x)$ only depends on $f(t, x, v)$ for the non-negative time $t \geq 0$.

The characteristics (trajectories) are determined by the Hamilton ODEs

$$
\frac{d}{d s}\left[\begin{array}{l}
X^{f}(s ; t, x, v) \\
V^{f}(s ; t, x, v)
\end{array}\right]=\left[\begin{array}{c}
V^{f}(s ; t, x, v) \\
-\nabla_{x} \phi_{f}\left(s, X^{f}(s ; t, x, v)\right)
\end{array}\right],
$$

for $-\infty<s, t<\infty$ with $\left(X^{f}(t ; t, x, v), V^{f}(t ; t, x, v)\right)=(x, v)$. 
For $(t, x, v) \in \mathbb{R} \times \Omega \times \mathbb{R}^{3}$, we define the backward exit time $t_{\mathbf{b}}^{f}(t, x, v)$ as

$$
t_{\mathbf{b}}^{f}(t, x, v):=\sup \left\{s \geq 0: X^{f}(\tau ; t, x, v) \in \Omega \text { for all } \tau \in(t-s, t)\right\} .
$$

Furthermore, we define $x_{\mathbf{b}}^{f}(t, x, v):=X^{f}\left(t-t_{\mathbf{b}}^{f}(t, x, v) ; t, x, v\right)$ and $v_{\mathbf{b}}^{f}(t, x, v):=V^{f}\left(t-t_{\mathbf{b}}^{f}(t, x, v) ; t, x, v\right)$. We note that all the definitions of $X^{f}(s ; t, x, v)$ and $V^{f}(s ; t, x, v)$ only depend on $f(t, x, v)$ for a non-negative time and our extension (1.21). Hence our definition $t_{\mathbf{b}}^{f}(t, x, v)$ does not depend on $f(t, x, v)$ for $t<0$.

If we compel $f$ to solve (1.19) and (1.20) even for a negative time with $\phi_{f}$ defined for all $t \in \mathbb{R}$ as (1.21) then

$$
f(t, x, v)=g\left(t-t_{\mathbf{b}}^{f}(t, x, v), x_{\mathbf{b}}^{f}(t, x, v), v_{\mathbf{b}}^{f}(t, x, v)\right) \quad \text { for } t \geq t_{\mathbf{b}}^{f}(t, x, v) .
$$

From direct computations (see (2.24) and (2.25), derivatives have a singularity in general as

$$
\nabla_{x} f(t, x, v) \sim \nabla_{x} x_{\mathbf{b}}^{f}(t, x, v) \sim \frac{1}{n\left(x_{\mathbf{b}}^{f}(t, x, v)\right) \cdot v_{\mathbf{b}}^{f}(t, x, v)} .
$$

Inspired by this observation, we introduce the following notion.

Definition 1 (Kinetic Weight). Suppose $f(t, x, v)$ is given for $t \geq 0,(x, v) \in \bar{\Omega} \times \mathbb{R}^{3}$ and satisfies (1.18). We define $\phi_{f}(t, x)$ as a unique solution of (1.16) for $t \geq 0$ and extend $\phi_{f}(t, x)$ for a negative time as well by (1.21). Suppose $\left(X^{f}(s ; t, x, v), V^{f}(s ; t, x, v)\right)$ solves (1.22) and $t_{\mathbf{b}}^{f}$ is defined as (1.23). For $\varepsilon>0$, we define a kinetic weight as

$$
\begin{aligned}
\alpha_{f, \varepsilon}(t, x, v):= & \chi\left(\frac{t-t_{\mathbf{b}}^{f}(t, x, v)+\varepsilon}{\varepsilon}\right)\left|n\left(x_{\mathbf{b}}^{f}(t, x, v)\right) \cdot v_{\mathbf{b}}^{f}(t, x, v)\right| \\
& +\left[1-\chi\left(\frac{t-t_{\mathbf{b}}^{f}(t, x, v)+\varepsilon}{\varepsilon}\right)\right] .
\end{aligned}
$$

Here we use a smooth function $\chi: \mathbb{R} \rightarrow[0,1]$ satisfying

$$
\begin{array}{r}
\chi(\tau)=0, \tau \leq 0, \text { and } \chi(\tau)=1, \tau \geq 1, \\
\frac{d}{d \tau} \chi(\tau) \in[0,4] \text { for all } \tau \in \mathbb{R} .
\end{array}
$$

Note that $\alpha_{f, \varepsilon}(0, x, v)$ is solely determined by $\phi_{f}(t, x)=e^{-|t|} \phi_{f_{0}}(x)$ for $t \leq 0$, which only depends on $f_{0}$. Moreover, we have

$$
\alpha_{f, \varepsilon}(t, x, v)=|n(x) \cdot v| \text { on } \gamma_{-} .
$$

For the sake of simplicity, we could drop the superscription $f$ in $X^{f}, V^{f}, t_{\mathbf{b}}^{f}, x_{\mathbf{b}}^{f}, v_{\mathbf{b}}^{f}$ unless this could cause any confusion.

One of the crucial properties of the new kinetic weight in (1.25) is an invariance under the action of the Vlasov operator:

$$
\left[\partial_{t}+v \cdot \nabla_{x}-\nabla_{x} \phi_{f} \cdot \nabla_{v}\right] \alpha_{f, \varepsilon}(t, x, v)=0 .
$$

This is due to the fact that the characteristics solve a deterministic system (1.22) (See the proof in the appendix). This crucial invariant property under the Vlasov operator is one of the key points in our approach.

Importantly we note that several different versions of kinetic weight have been used, for convex domains, in [15] for $E \equiv 0$, and in [19, 23, 2] for $E \neq 0$ but when an extra favorable sign condition

$$
E \cdot n>0 \text { on } \partial \Omega
$$

is imposed (recall that $n$ is the outward normal at the boundary). When $E \equiv 0$, this weight takes a form of $\tilde{\alpha}$ in (3.32) which is basically equivalent to $\left\{\operatorname{dist}(x, \partial \Omega)^{2}+|n(x) \cdot v|^{2}+|v|^{2} \operatorname{dist}(x, \partial \Omega)\right\}^{1 / 2}$ (also see [15]). For $E \neq 0$, when the Vlasov operator hits $(\tilde{\alpha})^{2}$ the outcome contains $|v| \times \operatorname{dist}(x, \partial \Omega)$. This term can be bounded as

$$
\frac{1}{|v|} \times(\tilde{\alpha})^{2} \text { with a harmful factor } \frac{1}{|v|} \text {. }
$$

Clearly we lose control for small velocity. Such loss of control for small velocity is indeed a generic difficulty in the study of characteristics with external fields $E$ : as the velocity gets smaller, the curvature effect $|v|^{2}$ dist $(x, \partial \Omega)$ vanishes quadratically and therefore even small field can change the characteristics drastically when the velocity is small. While, the favorable sign condition prevents a possible bad behavior of trajectories of small velocity (an interior point can reach the boundary tangentially via characteristics). In fact, in this case, an extra term $(E \cdot n) \times \operatorname{dist}(x, \partial \Omega)$ can be added to $(\tilde{\alpha})^{2}$ (for example see the definition above Lemma 7 in $[2]$ ), and it leads a control of $|v| \times \operatorname{dist}(x, \partial \Omega)$ by $\frac{1}{E \cdot n} \times|v|(\tilde{\alpha})^{2}$. Unfortunately such approach fails in our case $\left.E \cdot n\right|_{\partial \Omega}=0$ as 
(1.10). Another problem of this weight is that, $\tilde{\alpha}$ is not exactly invariant under the transport operator (or Vlasov operator) but an extra $\langle v\rangle$-multiplier appears as

$$
\left|\left[\partial_{t}+v \cdot \nabla_{x}-\nabla_{x} \phi_{f} \cdot \nabla_{v}\right] \tilde{\alpha}\right| \lesssim\langle v\rangle \tilde{\alpha}
$$

Conceivably this causes a super-exponential growth in $\tilde{\alpha}$-weighted $W^{1, p}$ estimate, which seems quite hard to lead an asymptotical stability of $\mu$.

In this paper we establish an exponential asymptotical stability of $\mu$ when the potential satisfies zero Neumann boundary condition (1.10) (therefore no favorable sign condition of $E \cdot n$ at the boundary). For two species problem such a sign condition would not be helpful anymore since a favorable sign of electrons would be bad sign for ions (and vise versa). The new method of this paper, which does not require the favorable sign condition, would serve an effective machinery of two species problems in global well-posedness ([3]) and the hydrodynamic limit of various two species Vlasov-Boltzmann equation $([24,1])$.

1.2. Main Results. Construction of a unique global solution and proving its asymptotic stability of VPB in general domains has been a challenging open problem for any boundary condition. The main goal of this paper is to provide the first construction of a unique global strong solution of VPB system with the diffuse boundary condition when the domain is $C^{3}$ and convex. Moreover an asymptotic stability of the global Maxwellian $\mu$ is studied.

Here a $C^{3}$ domain means that for any $p \in \partial \Omega$, there exist sufficiently small $\delta_{1}>0, \delta_{2}>0$, and an one-to-one and onto $C^{3}$-map

$$
\begin{aligned}
\eta_{p}:\left\{x_{\|} \in \mathbb{R}^{2}:\left|x_{\|}\right|<\delta_{1}\right\} & \rightarrow \partial \Omega \cap B\left(p, \delta_{2}\right), \\
x_{\|}=\left(x_{\|, 1}, x_{\|, 2}\right) & \mapsto \eta_{p}\left(x_{\|, 1}, x_{\|, 2}\right) .
\end{aligned}
$$

A convex domain means that there exists $C_{\Omega}>0$ such that for all $p \in \partial \Omega$ and $\eta_{p}$ and for all $x_{\|}$in (1.29)

$$
\sum_{i, j=1}^{2} \zeta_{i} \zeta_{j} \partial_{i} \partial_{j} \eta_{p}\left(x_{\|}\right) \cdot n\left(x_{\|}\right) \leq-C_{\Omega}|\zeta|^{2} \text { for all } \zeta \in \mathbb{R}^{2} .
$$

We denote

$$
w_{\vartheta}(v)=e^{\vartheta|v|^{2}} \text {. }
$$

Theorem 1. Assume a bounded open $C^{3}$ domain $\Omega \subset \mathbb{R}^{3}$ is convex as (1.30). Let $0<\tilde{\vartheta}<\vartheta \ll 1$. Assume a compatibility condition

$$
f_{0}(x, v)=c_{\mu} \sqrt{\mu(v)} \int_{n(x) \cdot u>0} f_{0}(x, u) \sqrt{\mu(u)}\{n(x) \cdot u\} \mathrm{d} u \quad \text { on } \gamma_{-} .
$$

There exists a small constant $0<\varepsilon_{0} \ll 1$ such that for all $0<\varepsilon \leq \varepsilon_{0}$ if an initial datum $F_{0}=\mu+\sqrt{\mu} f_{0} \geq 0$ satisfies (1.13) and

and

$$
\left\|w_{\vartheta} f_{0}\right\|_{L^{\infty}\left(\bar{\Omega} \times \mathbb{R}^{3}\right)}<\varepsilon,
$$

$$
\left\|w_{\tilde{\vartheta}} \alpha_{f_{0}, \varepsilon}^{\beta} \nabla_{x, v} f_{0}\right\|_{L^{p}\left(\Omega \times \mathbb{R}^{3}\right)}<\varepsilon \quad \text { for } 3<p<6, \quad 1-\frac{2}{p}<\beta<\frac{2}{3},
$$

and

$$
\left\|w_{\tilde{\vartheta}} \nabla_{v} f_{0}\right\|_{L^{3}\left(\Omega \times \mathbb{R}^{3}\right)}<\infty,
$$

then there exists a unique global-in-time solution $\left(f, \phi_{f}\right)$ to (1.15), (1.16), 1.17) such that $F(t)=\mu+\sqrt{\mu} f(t) \geq$ 0. Moreover there exists $\lambda_{\infty}>0$ such that

$$
\sup _{t \geq 0} e^{\lambda_{\infty} t}\left\|w_{\vartheta} f(t)\right\|_{L^{\infty}\left(\bar{\Omega} \times \mathbb{R}^{3}\right)}+\sup _{t \geq 0} e^{\lambda_{\infty} t}\left\|\phi_{f}(t)\right\|_{C^{2}(\Omega)} \lesssim 1
$$

and, for some $C>0$,

and, for $0<\delta=\delta(p, \beta)$

$$
\left\|w_{\tilde{\vartheta}} \alpha_{f, \varepsilon}^{\beta} \nabla_{x, v} f(t)\right\|_{L^{p}\left(\Omega \times \mathbb{R}^{3}\right)} \lesssim e^{C t} \quad \text { for all } t \geq 0
$$

$$
\left\|\nabla_{v} f(t)\right\|_{L_{x}^{3}(\Omega) L_{v}^{1+\delta}\left(\mathbb{R}^{3}\right)} \lesssim_{t} 1 \text { for all } t \geq 0 .
$$

Furthermore, if $\left(f, \phi_{f}\right)$ and $\left(g, \phi_{g}\right)$ are both solutions to (1.15), 1.16), 1.17) then

$$
\|f(t)-g(t)\|_{L^{1+\delta}\left(\Omega \times \mathbb{R}^{3}\right)} \lesssim_{t}\|f(0)-g(0)\|_{L^{1+\delta}\left(\Omega \times \mathbb{R}^{3}\right)} \text { for all } t \geq 0 .
$$


Remark 1. A large class of functional spaces satisfy the condition (1.34). For example, any initial datum $f_{0}$, whose weak derivatives $\nabla_{x, v} f_{0}$ are small in $L^{p}\left(\Omega \times \mathbb{R}^{3}\right)$ for some $p>3$, and $\left|\nabla_{x, v} f_{0}\right|$ decays fast enough as $|v| \rightarrow \infty$, satisfies 1.34).

Remark 2. We note that an exponential growth bound of (1.37) is a stronger result than the result of [15]. In [15, the upper bound has a super-exponential growth $e^{C t^{2}}$ even in the absence of an external potential. The exponential growth of (1.37) is crucially used (in an interpolation with an exponential decay of $\phi_{f}(t)$ in $C^{1,1-\varepsilon}$ for $0<\varepsilon \ll 1$, Lemma 1) to obtain a decay of $\phi_{f}(t)$ in $C^{2}$. And this decay-in-time of $\phi_{f}$ in $C^{2}$ is one of the crucial ingredient to construct a global-in-time solution.

Remark 3. As far as the authors know, Theorem 1 provides the first unique global-in-time solution to the Vlasov-Poisson-Boltzmann system in bounded domains with physical boundary condition. Moreover, the result of (1.36) is the first proof of asymptotic stability toward the global Maxwellian for Vlasov-Poisson-Boltzmann system with physical boundary. Also the result of (1.37) and (1.38) are the first regularity results of VlasovPoisson-Boltzmann system in bounded domains with a physical boundary.

Remark 4. We note that the norm in (1.37) is nonlinear in $f$. In the construction of solutions via a sequence argument this nonlinearity causes subtle issue on the convergence (see the proof of Theorem 7 )

1.3. Nonlinear-Normed Energy Estimates and an Interpolation. In the energy-type estimate of $\nabla_{x, v} f$ in $\alpha_{f, \varepsilon}^{\beta}$-weighted $L^{p}$-norm, the operator $v \cdot \nabla_{x}$ causes a boundary term to be controlled:

$$
\int_{0}^{t} \int_{\gamma_{-}}\left|\alpha_{f, \varepsilon}^{\beta} \nabla_{x, v} f\right|^{p} \mathrm{~d} s .
$$

Considering the singularity of (1.24) and (1.27), this integrand is integrable if

$$
\beta>\frac{p-2}{p} \text { so that }|n \cdot v|^{p \beta-p+1} \in L_{l o c}^{1}\left(\mathbb{R}^{3}\right) .
$$

On the other hand, in the bulk, we have two terms to be controlled:

$$
\int_{0}^{t} \iint_{\Omega \times \mathbb{R}^{3}} \nabla_{x}^{2} \phi_{f} \nabla_{v} f \alpha_{f, \varepsilon}^{p \beta}\left|\nabla_{x, v} f\right|^{p-1}
$$

and

$$
\int_{0}^{t} \iint_{\Omega \times \mathbb{R}^{3}} K \nabla_{x, v} f \alpha_{f, \varepsilon}^{p \beta}\left|\nabla_{x, v} f\right|^{p-1}
$$

To handle (1.41) we need a bound of $\phi_{f}(t)$ in $C_{x}^{2}$. Unfortunately such estimate is a boarder line case of the well-known Schauder elliptic regularity theory in (1.16) when $\int_{\mathbb{R}^{3}} f \sqrt{\mu} \mathrm{d} v$ is merely continuous or bounded. A key observation is that, for $\frac{1}{p}+\frac{1}{p^{*}}=1$, we have

$$
\left\|\int_{\mathbb{R}^{3}} \nabla_{x} f \sqrt{\mu} \mathrm{d} v\right\|_{L_{x}^{p}(\Omega)} \lesssim \sup _{x}\left\|\frac{\sqrt{\mu}}{\alpha_{f, \varepsilon}^{\beta}}\right\|_{L^{p^{*}}\left(\mathbb{R}^{3}\right)}\left\|\alpha_{f, \varepsilon}^{\beta} \nabla_{x} f\right\|_{L^{p}\left(\Omega \times \mathbb{R}^{3}\right)},
$$

which leads $C^{2,0+}$-bound of $\phi_{f}$ by the Morrey inequality for $p>3$ as long as

$$
\alpha_{f, \varepsilon}^{-\beta p^{*}} \in L_{l o c}^{1}\left(\left\{v \in \mathbb{R}^{3}\right\}\right) \text { for some } \beta p^{*}>\frac{p-2}{p-1} .
$$

We note that $\phi_{f}(t)$ has an exponential decay in weaker Hölder spaces $C^{1,1-}$ if $f$ decays exponentially in $L^{\infty}$. As long as the bound (1.43) grows at most exponentially in time,

$$
\text { an exponential decay of } \phi_{f}(t) \text { in } C_{x}^{2}
$$

can be verified through the following interpolation in Hölder spaces.

Lemma 1. Assume $\Omega \subset \mathbb{R}^{3}$ with a smooth boundary $\partial \Omega$. For $0<D_{1}<1,0<D_{2}<1$, and $\Lambda_{0}>0$,

$$
\begin{aligned}
\left\|\nabla_{x}^{2} \phi(t)\right\|_{L^{\infty}(\Omega)} \lesssim \Omega_{\Omega, D_{1}, D_{2}} & e^{D_{1} \Lambda_{0} t}\|\phi(t)\|_{C^{1,1-D_{1}}(\Omega)} \\
& +e^{-D_{2} \Lambda_{0} t}\|\phi(t)\|_{C^{2, D_{2}}(\Omega)} \quad \text { for all } t \geq 0 .
\end{aligned}
$$

The proof of this lemma is given at Section 8 
1.4. Desingularization via Mixing in Velocity. To prove (1.44), a major difficulty arises from the non-local feature of $\alpha_{f, \varepsilon}$, which is determined on the characteristics at $t-t_{\mathbf{b}}^{f}(t, x, v)$. We exam the integrability of $\alpha_{f, \varepsilon}^{-\beta p^{*}}$ by employing a change of variables

$$
v \mapsto\left(x_{\mathbf{b}}^{f}(t, x, v), t_{\mathbf{b}}^{f}(t, x, v)\right) .
$$

By the direct computation, the Jacobian is equivalent to (see (3.100)

$$
\begin{aligned}
& \frac{\left|t_{\mathbf{b}}^{f}\right|^{3}}{\alpha_{f, \varepsilon}(t, x, v)} \\
& \times \operatorname{det}\left[\operatorname{Id}_{3 \times 3}+\frac{1}{t_{\mathbf{b}}^{f}} \int_{t}^{t-t_{\mathbf{b}}^{f}} \int_{t}^{s} \nabla_{v} X^{f}(\tau) \nabla_{x}^{2} \phi_{f}\left(\tau ; X^{f}(\tau)\right) \mathrm{d} \tau \mathrm{d} s\right],
\end{aligned}
$$

where $X^{f}(\tau)=X^{f}(\tau ; t, x, v)$.

Importantly we note that, for having a uniform-in-time positive lower bound of (1.48), it is necessary to have

$$
\left|\nabla_{v} X^{f}(\tau ; t, x, v)\right| \lesssim|t-\tau| \text { for all } \tau \leq t .
$$

In the presence of a time-dependent potential we have a non-autonomous system from (1.22):

$$
\frac{d}{d s}\left[\begin{array}{c}
\nabla_{v} X^{f}(s ; t, x, v) \\
\nabla_{v} V^{f}(s ; t, x, v)
\end{array}\right]=\left[\begin{array}{cc}
0_{3 \times 3} & \operatorname{Id}_{3 \times 3} \\
-\nabla_{x}^{2} \phi_{f}\left(s, X^{f}(s)\right) & 0_{3 \times 3}
\end{array}\right]\left[\begin{array}{l}
\nabla_{v} X^{f}(s ; t, x, v) \\
\nabla_{v} V^{f}(s ; t, x, v)
\end{array}\right] .
$$

Using an exponential decay from (1.45), we prove (1.49) in Lemma 10 and therefore conclude (1.47) as long as (1.45) can be verified.

Applying the change of variables (1.47), $\alpha_{f, \varepsilon}$-factor in the Jacobian (1.48) cancels out the singularity in (1.44) and leads $\alpha_{f, \varepsilon}^{1-\beta p^{*}} /\left(t_{\mathbf{b}}^{f}\right)^{3}$ instead. Then we carefully use a lower bound of $t_{\mathbf{b}}^{f} \gtrsim \frac{\left|x_{\mathbf{b}}^{f}-x\right|}{\max \left|V^{f}\right|}$ and a bound $\alpha_{f, \varepsilon} \lesssim \frac{\left|\left(x-x_{\mathbf{b}}^{f}\right) \cdot n\left(x_{\mathbf{b}}^{f}\right)\right|}{t_{\mathbf{b}}^{f}}($ see $(\underline{3.18}))$ to have

$$
\begin{aligned}
& \int_{|v| \lesssim 1} \alpha_{f, \varepsilon}^{-\beta p^{*}} \mathrm{~d} v \\
\lesssim & \int_{\text {boundary }} \frac{\left|\left(x-x_{\mathbf{b}}^{f}\right) \cdot n\left(x_{\mathbf{b}}^{f}\right)\right|^{1-\beta p^{*}}}{\left|x-x_{\mathbf{b}}^{f}\right|^{3-\beta p^{*}}} \mathrm{~d} x_{\mathbf{b}}^{f}+\operatorname{good} \text { terms }<\infty,
\end{aligned}
$$

which turns to be bounded as long as $\beta p^{*}<1$.

This estimate is good to control (1.41) but we have to restrict a range of $p$ due to $\frac{1}{|v-u|}$-singularity of $K$ in (1.42) (see (2.4)). We bound $K\left(\frac{1}{\alpha_{f, \varepsilon}^{\beta}} \alpha_{f, \varepsilon}^{\beta} \nabla_{x, v} f\right)$ by $\left\|\frac{1}{|v-u|} \frac{1}{\alpha_{f, \varepsilon}^{\beta}(u)}\right\|_{L_{u}^{p^{*}}} \times\left\|\alpha_{f, \varepsilon}^{\beta} \nabla_{x, v} f\right\|_{L_{u}^{p}}$. Viewing $\left\|\frac{1}{|v-u|} \frac{1}{\alpha_{f, \varepsilon}^{\beta}(u)}\right\|_{L_{u}^{p^{*}}}$ as $\left|\frac{1}{|v-\cdot|^{p^{*}}} * \frac{1}{\alpha_{f, \varepsilon}(\cdot)^{\beta p^{*}}}\right|^{1 / p^{*}}$ we apply the Hardy-Littlewood-Sobolev inequality to have

$$
\left\|\frac{1}{|v-\cdot|^{p^{*}}} * \frac{1}{\alpha_{f, \varepsilon}(\cdot)^{\beta p^{*}}}\right\|_{L^{p / p^{*}}} \lesssim\left\|\frac{1}{\alpha_{f, \varepsilon}^{\beta}}\right\|_{L_{l o c}^{3 / 2}}+\operatorname{good} \text { terms. }
$$

This causes another restriction $\beta<\frac{2}{3}$ from (1.51) and then $p<6$ from (1.40).

1.5. $L^{\infty}$-Estimate. Finally we use an $L^{2}-L^{\infty}$ bootstrap argument to derive an exponential decay of $f$ in $L^{\infty}$. The key of this process is to derive a positive lower bound of

$$
\operatorname{det}\left(-(t-s) \operatorname{Id}_{3 \times 3}-\int_{t}^{s} \int_{t}^{\tau} \frac{\partial X^{f}\left(\tau^{\prime}\right)}{\partial v} \nabla_{x}^{2} \phi\left(\tau^{\prime}, X^{f}\left(\tau^{\prime}\right)\right) \mathrm{d} \tau^{\prime} \mathrm{d} \tau\right),
$$

except for a small set of $s$. Here $X^{f}\left(\tau^{\prime}\right)=X^{f}\left(\tau^{\prime} ; t, x, v\right)$. Again as (1.48) it is crucial to verify (1.45) and (1.49) for obtaining a uniform-in-time positive lower bound of (1.53). Finally we can close the estimates by proving an exponential growth bound of $\left\|\alpha_{f, \varepsilon}^{\beta} \nabla_{x, v} f\right\|_{L^{p}\left(\Omega \times \mathbb{R}^{3}\right)}$ from the Gronwall inequality and an exponential decay of $f$ in $L^{\infty}$ and therefore achieve (1.45) by Lemma 1 
1.6. A Priori $L_{x}^{3} L_{v}^{1+}$-Estimate of $\nabla_{v} f$ and $L^{1+}$-Stability. For constructing a solution and proving its uniqueness, we need some stability estimate of the difference of the solutions $f-g$. The difficulty comes from the term of $\nabla_{x} \phi_{f} \cdot \nabla_{v} f$. To prove $L^{q}$-stability for $q=1+$ we have, by Sobolev embedding $\nabla_{x} \phi_{f-g} \in W^{1, q}(\Omega) \subset$ $L(\Omega)^{\frac{3 q}{3-q}}$,

$$
\begin{aligned}
& \iint\left|\nabla_{x} \phi_{f-g} \cdot \nabla_{v} f\right||f-g|^{q-1} \\
\lesssim & \left\|\nabla_{x} \phi_{f-g}\right\|_{L_{x}^{3-q}}\|\| \nabla_{v} f\left\|_{L_{v}^{q}}\right\|_{L_{x}^{3}}\left\||f-g|^{q-1}\right\|_{L_{x, v}^{\frac{q}{q-1}}} .
\end{aligned}
$$

We note that $\nabla_{v} f$ is bounded at $\gamma_{-}$, from the boundary condition (1.17). However the equation of $\nabla_{v} f$ has $\nabla_{x} f$ as a forcing term. Therefore the key term to bound \|\|$\nabla_{v} f\left\|_{L_{v}^{q}}\right\|_{L_{x}^{3}}$ for $q=1+$ is

$$
\begin{aligned}
& \|\| \int_{0}^{t} \nabla_{x} f(s, X(s ; t, x, v), V(s ; t, x, v)) \mathrm{d} s\left\|_{L_{v}^{1+}}\right\|_{L_{x}^{3}} \\
\lesssim & \int_{0}^{t}\left\|\alpha_{f, \varepsilon}^{-\beta}\right\|_{L_{x}^{\infty} L_{v}^{\frac{p}{p-1}}+}\left\|\alpha_{f, \varepsilon}^{\beta} \nabla_{x} f\right\|_{L_{x, v}^{p}} \text { for } p>3 .
\end{aligned}
$$

From (1.40), $\beta\left(\frac{p}{p-1}+\right)<1$ and therefore $\left\|\alpha_{f, \varepsilon}^{-\beta}\right\|_{L_{x}^{\infty} L_{v}^{\frac{p}{p-1}}+}$ is bounded from (1.44).

\section{Preliminary and In-Flow Problems}

We define standard notions in (1.15). For the hard sphere cross section (1.2) and the global Maxwellian (1.4),

$$
L f:=\frac{1}{\sqrt{\mu}}[Q(\mu, \sqrt{\mu} f)+Q(\sqrt{\mu} f, \mu)]:=\nu(v) f-K f .
$$

Here the collision frequency is defined as

$$
\nu(v):=\int_{\mathbb{S}^{2}} \int_{\mathbb{R}^{3}}|(v-u) \cdot \omega| \mu(u) \mathrm{d} u \mathrm{~d} \omega \sim\langle v\rangle:=\sqrt{1+|v|^{2}},
$$

and

$$
\begin{aligned}
K f & :=K_{2} f-K_{1} f \\
& :=\frac{1}{\sqrt{\mu}}\left[Q_{\text {gain }}(\mu, \sqrt{\mu} f)+Q_{\text {gain }}(\sqrt{\mu} f, \mu)\right]-\frac{1}{\sqrt{\mu}} Q_{\text {loss }}(\sqrt{\mu} f, \mu) \\
& :=\int_{\mathbb{R}^{3}} \mathbf{k}_{2}(v, u) f(u) \mathrm{d} u-\int_{\mathbb{R}^{3}} \mathbf{k}_{1}(v, u) f(u) \mathrm{d} u .
\end{aligned}
$$

It is well-known (See [20]) that, for some constants $C_{\mathbf{k}_{1}}>0$ and $C_{\mathbf{k}_{2}}>0$,

$$
\begin{aligned}
& \mathbf{k}_{1}(v, u)=C_{\mathbf{k}_{1}}|v-u| e^{-\frac{|v|^{2}+|u|^{2}}{4}}, \\
& \mathbf{k}_{2}(v, u)=C_{\mathbf{k}_{2}}|v-u|^{-1} e^{-\frac{|v-u|^{2}}{8}} e^{-\frac{|v|^{2}-\left.|u|^{2}\right|^{2}}{8|v-u|^{2}}} .
\end{aligned}
$$

The nonlinear operator is defined as

$$
\begin{aligned}
\Gamma\left(f_{1}, f_{2}\right) & :=\Gamma_{\text {gain }}\left(f_{1}, f_{2}\right)-\Gamma_{\text {loss }}\left(f_{1}, f_{2}\right) \\
& :=\frac{1}{\sqrt{\mu}} Q_{\text {gain }}\left(\sqrt{\mu} f_{1}, \sqrt{\mu} f_{2}\right)-\frac{1}{\sqrt{\mu}} Q_{\text {loss }}\left(\sqrt{\mu} f_{1}, \sqrt{\mu} f_{2}\right) .
\end{aligned}
$$

From now on, in this section, we prove basic estimates of initial-boundary problems of the transport equation in the presence of a time-dependent field $E(t, x)$

$$
\partial_{t} f+v \cdot \nabla_{x} f+E \cdot \nabla_{v} f+\psi f=H
$$

where $H=H(t, x, v)$ and $\psi=\psi(t, x, v) \geq 0$. We assume that $E$ is defined for all $t \in \mathbb{R}$. Throughout this section $(X(s ; t, x, v), V(s ; t, x, v))$ denotes the characteristic which is determined by (1.22) with replacing $-\nabla_{x} \phi_{f}$ by $E$.

Lemma 2. Assume that $\Omega$ is convex (1.30). Suppose that $\sup _{t}\|E(t)\|_{C_{x}^{1}}<\infty$ and

$$
n(x) \cdot E(t, x)=0 \text { for } x \in \partial \Omega \text { and for all } t \text {. }
$$


Assume $(t, x, v) \in \mathbb{R}_{+} \times \bar{\Omega} \times \mathbb{R}^{3}$ and $t+1 \geq t_{\mathbf{b}}(t, x, v)$. If $x \in \partial \Omega$ then we further assume that $n(x) \cdot v>0$. Then we have

$$
n\left(x_{\mathbf{b}}(t, x, v)\right) \cdot v_{\mathbf{b}}(t, x, v)<0 .
$$

Proof. Step 1. Note that we can locally parametrize the trajectory (see Lemma 15 in [15] or 28] for details). We consider local parametrization (1.29). We drop the subscript $p$ for the sake of simplicity. If $X(s ; t, x, v)$ is near the boundary then we can define $\left(X_{n}, X_{\|}\right)$to satisfy

$$
X(s ; t, x, v)=\eta\left(X_{\|}(s ; t, x, v)\right)+X_{n}(s ; t, x, v)\left[-n\left(X_{\|}(s ; t, x, v)\right)\right] .
$$

For the normal velocity we define

$$
V_{n}(s ; t, x, v):=V(s ; t, x, v) \cdot\left[-n\left(X_{\|}(s ; t, x, v)\right)\right] .
$$

We define $V_{\|}$tangential to the level set $\left(\eta\left(X_{\|}\right)+X_{n}\left(-n\left(X_{\|}\right)\right)\right)$for fixed $X_{n}$. Note that

$$
\frac{\partial\left(\eta\left(x_{\|}\right)+x_{n}\left(-n\left(x_{\|}\right)\right)\right)}{\partial x_{\|, i}} \perp n\left(x_{\|}\right) \text {for } i=1,2 .
$$

We define $\left(V_{\|, 1}, V_{\|, 2}\right)$ as

$$
V_{\|, i}:=\left(V-V_{n}\left[-n\left(X_{\|}\right)\right]\right) \cdot\left(\frac{\partial \eta\left(X_{\|}\right)}{\partial x_{\|, i}}+X_{n}\left[-\frac{\partial n\left(X_{\|}\right)}{\partial x_{\|, i}}\right]\right) .
$$

Therefore we obtain

$$
V(s ; t, x, u)=V_{n}\left[-n\left(X_{\|}\right)\right]+V_{\|} \cdot \nabla_{x_{\|}} \eta\left(X_{\|}\right)-X_{n} V_{\|} \cdot \nabla_{x_{\|}} n\left(X_{\|}\right) .
$$

Directly we have

$$
\dot{X}(s ; t, x, u)=\dot{X}_{\|} \cdot \nabla_{x_{\|}} \eta\left(X_{\|}\right)+\dot{X}_{n}\left[-n\left(X_{\|}\right)\right]-X_{n} \dot{X}_{\|} \cdot \nabla_{x_{\|}} n\left(X_{\|}\right) .
$$

Comparing coefficients of normal and tangential components, we obtain that

$$
\dot{X}_{n}(s ; t, x, v)=V_{n}(s ; t, x, v), \quad \dot{X}_{\|}(s ; t, x, v)=V_{\|}(s ; t, x, v) .
$$

On the other hand, from (2.12),

$$
\begin{aligned}
\dot{V}(s)= & \dot{V}_{n}\left[-n\left(X_{\|}\right)\right]-V_{n} \nabla_{x_{\|}} n\left(X_{\|}\right) \dot{X}_{\|}+V_{\|} \cdot \nabla_{x_{\|}}^{2} \eta\left(X_{\|}\right) \dot{X}_{\|} \\
& +\dot{V}_{\|} \cdot \nabla_{x_{\|}} \eta\left(X_{\|}\right)-\dot{X}_{n} \nabla_{x_{\|}} n\left(X_{\|}\right) V_{\|}-X_{n} \nabla_{x_{\|}} n\left(X_{\|}\right) \dot{V}_{\|} \\
& -X_{n} V_{\|} \cdot \nabla_{x_{\|}}^{2} n\left(X_{\|}\right) \dot{X}_{\|} .
\end{aligned}
$$

From (2.14) $\cdot\left[-n\left(X_{\|}\right)\right],(2.13)$, and $\dot{V}=E$, we obtain that

$$
\begin{aligned}
\dot{V}_{n}(s)= & {\left[V_{\|}(s) \cdot \nabla^{2} \eta\left(X_{\|}(s)\right) \cdot V_{\|}(s)\right] \cdot n\left(X_{\|}(s)\right) } \\
& +E(s, X(s)) \cdot\left[-n\left(X_{\|}(s)\right)\right] \\
& -X_{n}(s)\left[V_{\|}(s) \cdot \nabla^{2} n\left(X_{\|}(s)\right) \cdot V_{\|}(s)\right] \cdot n\left(X_{\|}(s)\right) .
\end{aligned}
$$

Step 2. We prove (2.8) by the contradiction argument. Assume we choose $(t, x, v)$ satisfying the assumptions of Lemma 2, Let us assume

$$
X_{n}\left(t-t_{\mathbf{b}} ; t, x, v\right)+V_{n}\left(t-t_{\mathbf{b}} ; t, x, v\right)=0 .
$$

First we choose $0<\varepsilon \ll 1$ such that $X_{n}(s ; t, x, v) \ll 1$ and

$$
V_{n}(s ; t, x, v) \geq 0 \text { for } t-t_{\mathbf{b}}(t, x, v)<s<t-t_{\mathbf{b}}(t, x, v)+\varepsilon .
$$

The sole case that we cannot choose such $\varepsilon>0$ is when there exists $0<\delta \ll 1$ such that $V_{n}(s ; t, x, v)<0$ for all $s \in\left(t-t_{\mathbf{b}}(t, x, v), t-t_{\mathbf{b}}(t, x, v)+\delta\right)$. But from (2.13) for $s \in\left(t-t_{\mathbf{b}}(t, x, v), t-t_{\mathbf{b}}(t, x, v)+\delta\right)$

$$
\begin{aligned}
0 & \leq X_{n}(s ; t, x, v) \\
& =X_{n}\left(t-t_{\mathbf{b}}(t, x, v) ; t, x, v\right)+\int_{t-t_{\mathbf{b}}(t, x, v)}^{s} V_{n}(\tau ; t, x, v) \mathrm{d} \tau<0 .
\end{aligned}
$$

Now with $\varepsilon>0$ in (2.17), temporarily we define that $t_{*}:=t-t_{\mathbf{b}}(t, x, v)+\varepsilon, x_{*}=X\left(t-t_{\mathbf{b}}(t, x, v)+\varepsilon ; t, x, v\right)$, and $v_{*}=V\left(t-t_{\mathbf{b}}(t, x, v)+\varepsilon ; t, x, v\right)$. Then $\left(X_{n}(s ; t, x, v), X_{\|}(s ; t, x, v)\right)=\left(X_{n}\left(s ; t_{*}, x_{*}, v_{*}\right), X_{\|}\left(s ; t_{*}, x_{*}, v_{*}\right)\right)$ and $\left(V_{n}(s ; t, x, v), V_{\|}(s ; t, x, v)\right)=\left(V_{n}\left(s ; t_{*}, x_{*}, v_{*}\right), V_{\|}\left(s ; t_{*}, x_{*}, v_{*}\right)\right)$. 
Now we consider the RHS of (2.15). From (1.30), the first term $\left[V_{\|}(s) \cdot \nabla^{2} \eta\left(X_{\|}(s)\right) \cdot V_{\|}(s)\right] \cdot n\left(X_{\|}(s)\right) \leq 0$. By an expansion and (2.7) we can bound the second term

$$
\begin{aligned}
& E(s, X(s)) \cdot n\left(X_{\|}(s)\right) \\
= & E\left(s, X_{n}(s), X_{\|}(s)\right) \cdot n\left(X_{\|}(s)\right) \\
= & E\left(s, 0, X_{\|}(s)\right) \cdot n\left(X_{\|}(s)\right)+\|E(s)\|_{C_{x}^{1}} O\left(\left|X_{n}(s)\right|\right) \\
= & \|E(s)\|_{C_{x}^{1}} O\left(\left|X_{n}(s)\right|\right) .
\end{aligned}
$$

From (1.22) and assumptions of Lemma 2

$$
\left|V_{\|}(s ; t, x, v)\right| \leq|v|+t_{\mathbf{b}}(t, x, v)\|E\|_{\infty} \leq|v|+(1+t)\|E\|_{\infty} .
$$

Combining the above results with (2.15), we conclude that

$$
\dot{V}_{n}\left(s ; t_{*}, x_{*}, v_{*}\right) \lesssim\left(|v|+(1+t)\|E\|_{\infty}\right)^{2} X_{n}\left(s ; t_{*}, x_{*}, v_{*}\right),
$$

and hence from (2.13) for $t-t_{\mathbf{b}}(t, x, v) \leq s \leq t_{*}$

$$
\begin{aligned}
& \frac{d}{d s}\left[X_{n}\left(s ; t_{*}, x_{*}, v_{*}\right)+V_{n}\left(s ; t_{*}, x_{*}, v_{*}\right)\right] \\
\lesssim & \left(|v|+(1+t)\|E\|_{\infty}\right)^{2}\left[X_{n}\left(s ; t_{*}, x_{*}, v_{*}\right)+V_{n}\left(s ; t_{*}, x_{*}, v_{*}\right)\right] .
\end{aligned}
$$

By the Gronwall inequality and (2.16), for $t-t_{\mathbf{b}}(t, x, v) \leq s \leq t_{*}$

$$
\begin{aligned}
& {\left[X_{n}\left(s ; t_{*}, x_{*}, v_{*}\right)+V_{n}\left(s ; t_{*}, x_{*}, v_{*}\right)\right] } \\
\lesssim & {\left[X_{n}\left(t-t_{\mathbf{b}}(t, x, u)\right)+V_{n}\left(t-t_{\mathbf{b}}(t, x, u)\right)\right] e^{\left.C \varepsilon\left(|v|+(1+t)\|E\|_{\infty}\right)^{2}\right)} } \\
= & 0 .
\end{aligned}
$$

From (2.17) we conclude that $X_{n}(s ; t, x, v) \equiv 0$ and $V_{n}(s ; t, x, v) \equiv 0$ for all $s \in\left[t-t_{\mathbf{b}}(t, x, u), t-t_{\mathbf{b}}(t, x, u)+\varepsilon\right]$. We can continue this argument successively to deduce that $X_{n}(s ; t, x, v) \equiv 0$ and $V_{n}(s ; t, x, v) \equiv 0$ for all $s \in\left[t-t_{\mathbf{b}}(t, x, v), t\right]$. Therefore $x_{n}=0=v_{n}$ which implies $x \in \partial \Omega$ and $n(x) \cdot v=0$. This is a contradiction since we chose $n(x) \cdot v>0$ if $x \in \partial \Omega$.

Lemma 3. For fixed $t$, a map

$$
(x, v) \in \Omega \times \mathbb{R}^{3} \mapsto\left(t-t_{\mathbf{b}}(t, x, v), x_{\mathbf{b}}(t, x, v), v_{\mathbf{b}}(t, x, v)\right) \in \mathbb{R} \times \gamma_{-}
$$

is one-to-one and

$$
\begin{aligned}
& \left|\operatorname{det}\left(\frac{\partial\left(t-t_{\mathbf{b}}(t, x, v), x_{\mathbf{b}}(t, x, v), v_{\mathbf{b}}(t, x, v)\right)}{\partial(x, v)}\right)\right| \\
& =\frac{1}{\left|v_{\mathbf{b}}(t, x, v) \cdot n\left(x_{\mathbf{b}}(t, x, v)\right)\right|} \text {. }
\end{aligned}
$$

Also, a map

$$
(t, x, v) \in \mathbb{R} \times \gamma_{+} \mapsto\left(t-t_{\mathbf{b}}(t, x, v), x_{\mathbf{b}}(t, x, v), v_{\mathbf{b}}(t, x, v)\right) \in \mathbb{R} \times \gamma_{-}
$$

is one-to-one and

$$
\begin{aligned}
= & \left|\operatorname{det}\left(\frac{\partial\left(t-t_{\mathbf{b}}(t, x, v), x_{\mathbf{b}}(t, x, v), v_{\mathbf{b}}(t, x, v)\right)}{\partial(t, x, v)}\right)\right| \\
\left|n\left(x_{\mathbf{b}}(t, x, v)\right) \cdot v_{\mathbf{b}}(t, x, v)\right| &
\end{aligned}
$$

Proof. Both maps (2.20) and (2.22) are clearly one-to-one since the characteristics solve (1.22), and hence are deterministic.

From (1.29) we denote $\eta\left(x_{\mathbf{b}, \|}(t, x, v)\right)=\eta\left(x_{\mathbf{b}, 1}(t, x, v), x_{\mathbf{b}, 2}(t, x, v)\right)=x_{\mathbf{b}}(t, x, v)$. We use the notations $\partial_{s} X=\frac{\partial X(s ; t, x, v)}{\partial s}, \partial_{t} X=\frac{\partial X(s ; t, x, v)}{\partial t}, \partial_{x_{i}} X=\frac{\partial X(s ; t, x, v)}{\partial x_{i}}$, and $\partial_{v_{i}} X=\frac{\partial X(s ; t, x, v)}{\partial v_{i}}$. Then

$$
\begin{aligned}
& \partial_{x_{i}} t_{\mathbf{b}}(t, x, v)=\frac{\partial_{x_{i}} X\left(t-t_{\mathbf{b}}(t, x, v) ; t, x, v\right) \cdot n\left(x_{\mathbf{b}}(t, x, v)\right)}{v_{\mathbf{b}}(t, x, v) \cdot n\left(x_{\mathbf{b}}(t, x, v)\right)}, \\
& \partial_{v_{i}} t_{\mathbf{b}}(t, x, v)=\frac{\partial_{v_{i}} X\left(t-t_{\mathbf{b}}(t, x, v) ; t, x, v\right) \cdot n\left(x_{\mathbf{b}}(t, x, v)\right)}{v_{\mathbf{b}}(t, x, v) \cdot n\left(x_{\mathbf{b}}(t, x, v)\right)} .
\end{aligned}
$$


From (1.22)

$$
\begin{aligned}
\nabla_{x, v} x_{\mathbf{b}}(t, x, v) & =\nabla_{x, v}\left[X\left(t-t_{\mathbf{b}} ; t, x, v\right)\right] \\
& =\nabla_{x, v} t_{\mathbf{b}} v_{\mathbf{b}}+\nabla_{x, v} X\left(t-t_{\mathbf{b}} ; t, x, v\right) \\
\nabla_{x, v} v_{\mathbf{b}}(t, x, v) & =\nabla_{x, v}\left[V\left(t-t_{\mathbf{b}} ; t, x, v\right)\right] \\
& =\nabla_{x, v} t_{\mathbf{b}} E\left(t-t_{\mathbf{b}}, x_{\mathbf{b}}\right)+\nabla_{x, v} V\left(t-t_{\mathbf{b}} ; t, x, v\right)
\end{aligned}
$$

From (2.25)

$$
\begin{aligned}
& \frac{\partial\left(t-t_{\mathbf{b}}(t, x, v), x_{\mathbf{b}, \|}(t, x, v), v_{\mathbf{b}}(t, x, v)\right)}{\partial(x, v)} \\
= & {\left[\begin{array}{cc}
-\nabla_{x} t_{\mathbf{b}} & -\nabla_{v} t_{\mathbf{b}} \\
\left(-\nabla_{x} t_{\mathbf{b}} v_{\mathbf{b}}-\nabla_{x} X\right) \cdot \nabla\left(\eta^{-1}\right)_{1} & \left(-\nabla_{v} t_{\mathbf{b}} v_{\mathbf{b}}-\nabla_{v} X\right) \cdot \nabla\left(\eta^{-1}\right)_{1} \\
\left(-\nabla_{x} t_{\mathbf{b}} v_{\mathbf{b}}-\nabla_{x} X\right) \cdot \nabla\left(\eta^{-1}\right)_{2} & \left(-\nabla_{v} t_{\mathbf{b}} v_{\mathbf{b}}-\nabla_{v} X\right) \cdot \nabla\left(\eta^{-1}\right)_{2} \\
-\nabla_{x} t_{\mathbf{b}} E-\nabla_{x} V & -\nabla_{v} t_{\mathbf{b}} E-\nabla_{v} V
\end{array}\right], }
\end{aligned}
$$

where $\left(t_{\mathbf{b}}, X, V\right)=\left(t_{\mathbf{b}}(t, x, v), X\left(t-t_{\mathbf{b}}(t, x, v) ; t, x, v\right), V\left(t-t_{\mathbf{b}}(t, x, v) ; t, x, v\right)\right)$ and $E=E\left(t-t_{\mathbf{b}}, x_{\mathbf{b}}\right)$.

We multiply $-v_{\mathbf{b}} \cdot \nabla\left(\eta^{-1}\right)_{1}$ to the first row and add it to the second row. Then we multiply $-v_{\mathbf{b}} \cdot \nabla\left(\eta^{-1}\right)_{2}$ to the first row and add it to third second row. Next we multiply $E_{i}$ to the first row and add it to the $(i+3)$-row (elementary row operations). Then we obtain a matrix, with the same determinant as (2.26),

$$
\left[\begin{array}{cc}
-\nabla_{x} t_{\mathbf{b}} & -\nabla_{v} t_{\mathbf{b}} \\
-\nabla_{x} X \cdot \nabla\left(\eta^{-1}\right)_{1} & -\nabla_{v} X \cdot \nabla\left(\eta^{-1}\right)_{1} \\
-\nabla_{x} X \cdot \nabla\left(\eta^{-1}\right)_{2} & -\nabla_{v} X \cdot \nabla\left(\eta^{-1}\right)_{2} \\
-\nabla_{x} V & -\nabla_{v} V
\end{array}\right]
$$

Then using (2.24) we get another matrix with the same determinant

$$
\begin{aligned}
\operatorname{det}\left[\begin{array}{cc}
-\frac{n\left(x_{\mathbf{b}}\right)}{v_{\mathbf{b}} \cdot n\left(x_{\mathbf{b}}\right)} \cdot \nabla_{x} X & -\frac{n\left(x_{\mathbf{b}}\right)}{v_{\mathbf{b}} \cdot n\left(x_{\mathbf{b}}\right)} \cdot \nabla_{v} X \\
-\nabla_{x} X \cdot \nabla\left(\eta^{-1}\right)_{1} & -\nabla_{v} X \cdot \nabla\left(\eta^{-1}\right)_{1} \\
-\nabla_{x} X \cdot \nabla\left(\eta^{-1}\right)_{2} & -\nabla_{v} X \cdot \nabla\left(\eta^{-1}\right)_{2} \\
-\nabla_{x} V & -\nabla_{v} V
\end{array}\right] \\
=-\operatorname{det}\left[\begin{array}{cc}
\nabla_{x} X & \nabla_{x} V \\
\nabla_{v} X & \nabla_{v} V
\end{array}\right] \times \operatorname{det}\left[\begin{array}{ccc}
\frac{n\left(x_{\mathbf{b}}\right)}{v_{\mathbf{b}} \cdot n\left(x_{\mathbf{b}}\right)} & \nabla \eta^{-1} & 0 \\
0 & 0 & \mathrm{Id}_{3 \times 3}
\end{array}\right] .
\end{aligned}
$$

By the Liouville theorem det $\left[\begin{array}{cc}\nabla_{x} X & \nabla_{x} V \\ \nabla_{v} X & \nabla_{v} V\end{array}\right]=1$. Note that we can always assume $\partial_{x_{\|}, 1} \eta \cdot \partial_{x_{\|}, 2} \eta=0$ by reparametrization in (1.29). Then

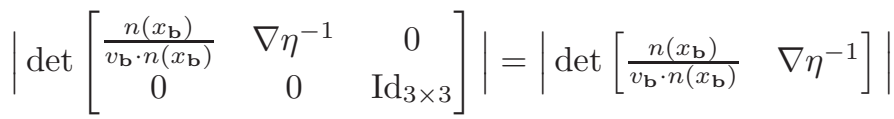

$$
\begin{aligned}
& =\frac{1}{\left|v_{\mathbf{b}} \cdot n\left(x_{\mathbf{b}}\right)\right|\left|\partial_{1} \eta\left(x_{\mathbf{b}}\right) \times \partial_{2} \eta\left(x_{\mathbf{b}}\right)\right|} .
\end{aligned}
$$

Therefore we conclude (2.21).

Next we consider (2.23). By direct computations and (2.25), (2.29)

$$
\begin{aligned}
& \frac{\partial\left(t-t_{\mathbf{b}}\left(t, \eta\left(x_{\|}\right), v\right), x_{\mathbf{b}, \|}\left(t, \eta\left(x_{\|}\right), v\right), v_{\mathbf{b}}\left(t, \eta\left(x_{\|}\right), v\right)\right)}{\partial\left(t, x_{\|}, v\right)} \\
& =\left[\begin{array}{cc}
1-\frac{\partial t_{\mathbf{b}}}{\partial t} & -\nabla_{x_{\|}} \eta\left(x_{\|}\right) \cdot \nabla_{x} t_{\mathbf{b}} \\
\left(\left(1-\frac{\partial t_{\mathbf{b}}}{\partial t}\right) v_{\mathbf{b}}+\partial_{t} X\right) \cdot \nabla\left(\eta^{-1}\right)_{1} & \left(\nabla_{x_{\|}} \eta \cdot\left(\nabla_{x_{\|}} t_{\mathbf{b}} v_{\mathbf{b}}+\nabla_{x_{\|}} X\right)\right) \cdot \nabla\left(\eta^{-1}\right)_{1} \\
\left(\left(1-\frac{\partial t_{\mathbf{b}}}{\partial t}\right) v_{\mathbf{b}}+\partial_{t} X\right) \cdot \nabla\left(\eta^{-1}\right)_{2} & \left(\nabla_{x_{\|}} \eta \cdot\left(\nabla_{x_{\|}} t_{\mathbf{b}} v_{\mathbf{b}}+\nabla_{x_{\|}} X\right)\right) \cdot \nabla\left(\eta^{-1}\right)_{2} \\
\left(1-\frac{\partial t_{\mathbf{b}}}{\partial t}\right) E\left(t-t_{\mathbf{b}}, x_{\mathbf{b}}\right)+\partial_{t} V & \nabla_{x_{\|}} \eta\left(x_{\|}\right) \cdot\left(\nabla_{x} t_{\mathbf{b}} E+\nabla_{x} V\right)
\end{array}\right. \\
& \left.\begin{array}{c}
-\nabla_{v} t_{\mathbf{b}} \\
\left(\nabla_{v} t_{\mathbf{b}} v_{\mathbf{b}}+\nabla_{v} X\right) \cdot \nabla\left(\eta^{-1}\right)_{1}
\end{array}\right] \\
& \left(\nabla_{v} t_{\mathbf{b}} v_{\mathbf{b}}+\nabla_{v} X\right) \cdot \nabla\left(\eta^{-1}\right)_{2} \\
& \nabla_{v} t_{\mathbf{b}} E+\nabla_{v} V
\end{aligned}
$$


By elementary row operations, we obtain

$$
\left[\begin{array}{ccc}
1-\frac{\partial t_{\mathbf{b}}}{\partial t} & -\nabla_{x_{\|}} \eta\left(x_{\|}\right) \cdot \nabla_{x} t_{\mathbf{b}} & -\nabla_{v} t_{\mathbf{b}} \\
\partial_{t} X \cdot \nabla\left(\eta^{-1}\right)_{1} & \left(\nabla_{x_{\|}} \eta \cdot \nabla_{x_{\|}} X\right) \cdot \nabla\left(\eta^{-1}\right)_{1} & \nabla_{v} X \cdot \nabla\left(\eta^{-1}\right)_{1} \\
\partial_{t} X \cdot \nabla\left(\eta^{-1}\right)_{2} & \left(\nabla_{x_{\|}} \eta \cdot \nabla_{x_{\|}} X\right) \cdot \nabla\left(\eta^{-1}\right)_{2} & \nabla_{v} X \cdot \nabla\left(\eta^{-1}\right)_{2} \\
\partial_{t} V & \nabla_{x_{\|}} \eta\left(x_{\|}\right) \cdot \nabla_{x} V & \nabla_{v} V
\end{array}\right] .
$$

Since the characteristics is deterministic, for either $0<\varepsilon \ll 1$ or $0<-\varepsilon \ll 1$ with $X\left(t+\varepsilon ; t, \eta\left(x_{\|}\right), v\right) \in \bar{\Omega}$,

$$
t_{\mathbf{b}}\left(t+\varepsilon, X\left(t+\varepsilon ; t, \eta\left(x_{\|}\right), v\right), V\left(t+\varepsilon ; t, \eta\left(x_{\|}\right), v\right)\right)=t_{\mathbf{b}}\left(t, \eta\left(x_{\|}\right), v\right)+\varepsilon .
$$

Differentiate (2.30) with respect to $\varepsilon$ and use (1.22), and then set $\varepsilon=0$ to have

$$
\left[\partial_{t}+v \cdot \nabla_{x}+E\left(t, \eta\left(x_{\|}\right)\right) \cdot \nabla_{v}\right] t_{\mathbf{b}}\left(t, \eta\left(x_{\|}\right), v\right)=1 .
$$

Also we have

$$
\begin{aligned}
& X\left(s ; t+\varepsilon, X\left(t+\varepsilon ; t, \eta\left(x_{\|}\right), v\right), V\left(t+\varepsilon ; t, \eta\left(x_{\|}\right), v\right)\right)=X\left(s ; t, \eta\left(x_{\|}\right), v\right), \\
& V\left(s ; t+\varepsilon, X\left(t+\varepsilon ; t, \eta\left(x_{\|}\right), v\right), V\left(t+\varepsilon ; t, \eta\left(x_{\|}\right), v\right)\right)=V\left(s ; t, \eta\left(x_{\|}\right), v\right) .
\end{aligned}
$$

We differentiate the above identity with respect to $\varepsilon$ and use (1.22), and then set $\varepsilon=0$ to have

$$
\begin{aligned}
& {\left[\partial_{t}+v \cdot \nabla_{x}+E\left(s, X\left(s ; t, \eta\left(x_{\|}\right), v\right)\right) \cdot \nabla_{v}\right] X\left(s ; t, \eta\left(x_{\|}\right), v\right)=0,} \\
& {\left[\partial_{t}+v \cdot \nabla_{x}+E\left(s, X\left(s ; t, \eta\left(x_{\|}\right), v\right)\right) \cdot \nabla_{v}\right] V\left(s ; t, \eta\left(x_{\|}\right), v\right)=0 .}
\end{aligned}
$$

From (2.29) and (2.31)

$$
\begin{aligned}
& \left(\underline{2.27)}=\left[\begin{array}{c}
v \cdot \nabla_{x} t_{\mathbf{b}}+E \cdot \nabla_{v} t_{\mathbf{b}} \\
\left(-v \cdot \nabla_{x} X-E \cdot \nabla_{v} X\right) \cdot \nabla\left(\eta^{-1}\right)_{1} \\
\left(-v \cdot \nabla_{x} X-E \cdot \nabla_{v} X\right) \cdot \nabla\left(\eta^{-1}\right)_{2} \\
-v \cdot \nabla_{x} V-E \cdot \nabla_{v} V
\end{array}\right.\right. \\
& \left.\begin{array}{cc}
-\nabla_{x_{\|}} \eta \cdot \nabla_{x} t_{\mathbf{b}} & -\nabla_{v} t_{\mathbf{b}} \\
\left(\nabla_{x_{\|}} \eta \cdot \nabla_{x_{\|}} X\right) \cdot \nabla\left(\eta^{-1}\right)_{1} & \nabla_{v} X \cdot \nabla\left(\eta^{-1}\right)_{1} \\
\left(\nabla_{x_{\|}} \eta \cdot \nabla_{x_{\|}} X\right) \cdot \nabla\left(\eta^{-1}\right)_{2} & \nabla_{v} X \cdot \nabla\left(\eta^{-1}\right)_{2} \\
\nabla_{x_{\|}} \eta\left(x_{\|}\right) \cdot \nabla_{x} V & \nabla_{v} V
\end{array}\right] .
\end{aligned}
$$

Now we multiply $v \cdot \partial_{x_{\|, i}} \eta$ to the $(i+1)$-column for $i=1,2$ and add these to the first column. Then we multiply $E_{i}$ to the $(i+3)$-column for $i=1,2,3$ and add these to the first column. And then we use (2.24) to get

$$
\left[\begin{array}{ccc}
\left(n\left(x_{\|}\right) \cdot v\right) \frac{n\left(x_{\|}\right) \cdot \nabla X\left(t-t_{\mathbf{b}}\right) \cdot n\left(x_{\mathbf{b}}\right)}{n\left(x_{\mathbf{b}}\right) \cdot v_{\mathbf{b}}} & -\nabla_{x_{\|}} \eta \cdot \frac{\nabla_{x} X \cdot n\left(x_{\mathbf{b}}\right)}{n\left(x_{\mathbf{b}}\right) \cdot v_{\mathbf{b}}} & -\frac{\nabla_{v} X \cdot n\left(x_{\mathbf{b}}\right)}{n\left(x_{\mathbf{b}}\right) \cdot v_{\mathbf{b}}} \\
-v \cdot n \partial_{n} X \cdot \nabla\left(\eta^{-1}\right)_{1} & \left(\nabla_{x_{\|}} \eta \cdot \nabla_{x_{\|}} X\right) \cdot \nabla\left(\eta^{-1}\right)_{1} & \nabla_{v} X \cdot \nabla\left(\eta^{-1}\right)_{1} \\
-v \cdot n \partial_{n} X \cdot \nabla\left(\eta^{-1}\right)_{2} & \left(\nabla_{x_{\|}} \eta \cdot \nabla_{x_{\|}} X\right) \cdot \nabla\left(\eta^{-1}\right)_{2} & \nabla_{v} X \cdot \nabla\left(\eta^{-1}\right)_{2} \\
-v \cdot n \partial_{n} V & \nabla_{x_{\|}} \eta\left(x_{\|}\right) \cdot \nabla_{x} V & \nabla_{v} V
\end{array}\right] .
$$

Therefore the determinant of (2.27) equals

$$
\begin{aligned}
\frac{n\left(x_{\|}\right) \cdot v}{n\left(x_{\mathbf{b}}\right) \cdot v_{\mathbf{b}}} & \times \operatorname{det}\left[\begin{array}{ccc}
n\left(x_{\|}\right) & \nabla_{x_{\|}} \eta & 0 \\
0 & 0 & \mathrm{Id}_{3 \times 3}
\end{array}\right] \operatorname{det}\left[\begin{array}{cc}
\nabla_{x} X & \nabla_{x} V \\
\nabla_{v} X & \nabla_{v} V
\end{array}\right] \\
& \times \operatorname{det}\left[\begin{array}{ccc}
n\left(x_{\mathbf{b}}\right) & \nabla\left(\eta^{-1}\right) & 0 \\
0 & 0 & \operatorname{Id}_{3 \times 3}
\end{array}\right]
\end{aligned}
$$

which implies (2.23).

Lemma 4. For fixed $s>0$ so that $t-t_{\mathbf{b}}(t, x, v)<s<t$,

$$
(t, x, v) \in[0, T] \times \gamma_{+} \mapsto(X(s ; t, x, v), V(s ; t, x, v)) \in \Omega \times \mathbb{R}^{3}
$$

is injective. For fixed $s>0$ so that $t<s<t+t_{\mathbf{f}}(t, x, v)$,

$$
(t, x, v) \in[0, T] \times \gamma_{-} \mapsto(X(s ; t, x, v), V(s ; t, x, v)) \in \Omega \times \mathbb{R}^{3}
$$

is also injective. For both maps (2.32) and (2.33),

$$
\left|\operatorname{det}\left(\frac{\partial(X(s ; t, x, v), V(s ; t, x, v))}{\partial(t, x, v)}\right)\right|=|n(x) \cdot v| .
$$


Moreover

$$
\begin{gathered}
(t, s, x, v) \in[0, T] \times\left\{-\min \left\{t, t_{\mathbf{b}}(t, x, v)\right\}<s<0\right\} \times \gamma_{+} \\
\mapsto(t+s, X(t+s ; t, x, v), V(t+s ; t, x, v)) \in[0, T] \times \Omega \times \mathbb{R}^{3}
\end{gathered}
$$

and

$$
\begin{aligned}
& (t, s, x, v) \in[0, T] \times\left\{0<s<\min \left\{t_{\mathbf{f}}(t, x, v), T-t\right\}\right\} \times \gamma_{-} \\
\mapsto & (t+s, X(t+s ; t, x, v), V(t+s ; t, x, v)) \in[0, T] \times \Omega \times \mathbb{R}^{3}
\end{aligned}
$$

are both injective, and for both maps (2.35) and 2.36)

$$
\left|\operatorname{det}\left(\frac{\partial(t+s, X(t+s ; t, x, v), V(t+s ; t, x, v))}{\partial(t, s, x, v)}\right)\right|=|n(x) \cdot v| .
$$

Proof. The maps are injective since the characteristics are a solution of (1.22).

For $x \in \partial \Omega$ we have $x=\eta\left(x_{\|}\right)$locally from (1.29). Then we compute (2.37),

$$
\begin{aligned}
& \frac{\partial\left(X\left(s ; t, \eta\left(x_{\|}\right), v\right), V\left(s ; t, \eta\left(x_{\|}\right), v\right)\right)}{\partial\left(t, x_{\|}, v\right)} \\
= & {\left[\begin{array}{lll}
\partial_{t} X\left(s ; t, \eta\left(x_{\|}\right), v\right) & \nabla_{x_{\|}} X\left(s ; t, \eta\left(x_{\|}\right), v\right) & \nabla_{v} X\left(s ; t, \eta\left(x_{\|}\right), v\right) \\
\partial_{t} V\left(s ; t, \eta\left(x_{\|}\right), v\right) & \nabla_{x_{\|}} V\left(s ; t, \eta\left(x_{\|}\right), v\right) & \nabla_{v} V\left(s ; t, \eta\left(x_{\|}\right), v\right)
\end{array}\right] } \\
= & {\left[\begin{array}{ll}
\partial_{t} X\left(s ; t, \eta\left(x_{\|}\right), v\right) & \nabla_{x_{\|}} \eta\left(x_{\|}\right) \cdot \nabla_{x} X\left(s ; t, \eta\left(x_{\|}\right), v\right) \\
\partial_{t} V\left(s ; t, \eta\left(x_{\|}\right), v\right) & \nabla_{x_{\|}} \eta\left(x_{\|}\right) \cdot \nabla_{x} V\left(s ; t, \eta\left(x_{\|}\right), v\right) \\
& \nabla_{v} X\left(s ; t, \eta\left(x_{\|}\right), v\right) \\
& \nabla_{v} V\left(s ; t, \eta\left(x_{\|}\right), v\right)
\end{array}\right] . }
\end{aligned}
$$

From (2.38) and (2.31),

$$
\begin{aligned}
(2.38)= & {\left[\begin{array}{cc}
\nabla_{x} X\left(s ; t, \eta\left(x_{\|}\right), v\right) & \nabla_{v} X\left(s ; t, \eta\left(x_{\|}\right), v\right) \\
\nabla_{x} V\left(s ; t, \eta\left(x_{\|}\right), v\right) & \nabla_{v} V\left(s ; t, \eta\left(x_{\|}\right), v\right)
\end{array}\right] } \\
& \times\left[\begin{array}{ccc}
-v & \partial_{x_{\|}} \eta\left(x_{\|}\right) & 0_{3 \times 3} \\
-E\left(s, X\left(s ; t, \eta\left(x_{\|}\right), v\right)\right) & 0_{3 \times 2} & \mathrm{Id}_{3 \times 3}
\end{array}\right] .
\end{aligned}
$$

From Liouville theorem, we conclude that

$$
\begin{aligned}
\text { (2.38) } & =\operatorname{det}\left[\begin{array}{ccc}
-v & \partial_{x_{\|}} \eta\left(x_{\|}\right) & 0_{3 \times 3} \\
-E\left(s, X\left(s ; t, \eta\left(x_{\|}\right), v\right)\right) & 0_{3 \times 2} & \operatorname{Id}_{3 \times 3}
\end{array}\right] \\
& =-v \cdot\left(\partial_{1} \eta\left(x_{\|}\right) \times \partial_{2} \eta\left(x_{\|}\right)\right) .
\end{aligned}
$$

Since the surface measure equals $\mathrm{d} S_{x}=\left|\partial_{1} \eta\left(x_{\|}\right) \times \partial_{2} \eta\left(x_{\|}\right)\right| \mathrm{d} x_{\|}$we conclude (2.34).

For (2.37) we compute

$$
\begin{aligned}
& \operatorname{det}\left(\frac{\partial\left(t+s, X\left(t+s ; t, \eta\left(x_{\|}\right), v\right), V\left(t+s ; t, \eta\left(x_{\|}\right), v\right)\right)}{\partial\left(t, s, x_{\|}, v\right)}\right) \\
&= \operatorname{det}\left[\begin{array}{cccc}
1 & 1 & 0 & 0 \\
\partial_{s} X(t+s)+\partial_{t} X(t+s) & \partial_{s} X(t+s) & \nabla_{x_{\|}} X(t+s) & \nabla_{v} X(t+s) \\
\partial_{s} V(t+s)+\partial_{t} V(t+s) & \partial_{s} V(t+s) & \nabla_{x_{\|}} V(t+s) & \nabla_{v} V(t+s)
\end{array}\right] \\
&=\operatorname{det}\left[\begin{array}{cccc}
0 & 1 & 0 & 0 \\
\partial_{t} X(t+s) & \partial_{s} X(t+s) & \nabla_{x_{\|}} X(t+s) & \nabla_{v} X(t+s) \\
\partial_{t} V(t+s) & \partial_{s} V(t+s) & \nabla_{x_{\|}} V(t+s) & \nabla_{v} V(t+s)
\end{array}\right] \\
&=\operatorname{det}\left[\begin{array}{llll}
\partial_{t} X(t+s) & \nabla_{x_{\|}} X(t+s) & \nabla_{v} X(t+s) \\
\partial_{t} V(t+s) & \nabla_{x_{\|}} V(t+s) & \nabla_{v} V(t+s)
\end{array}\right],
\end{aligned}
$$

which equals (2.38).

We define the forward exit time

$$
t_{\mathbf{f}}(t, x, v):=\sup \{s \geq 0: X(\tau ; t, x, v) \in \Omega \text { for all } \tau \in(t, t+s)\} .
$$


Lemma 5. Suppose $h(t, x, v) \in L^{1}\left([0, T] \times \Omega \times \mathbb{R}^{3}\right)$. Then

$$
\begin{aligned}
& \int_{0}^{T} \iint_{\Omega \times \mathbb{R}^{3}} h(t, x, v) \mathrm{d} v \mathrm{~d} x \mathrm{~d} t \\
& =\iint_{\Omega \times \mathbb{R}^{3}} \int_{-\min \left\{T, t_{\mathbf{b}}(T, x, v)\right\}}^{0} \times h(T+s, X(T+s ; T, x, v), V(T+s ; T, x, v)) \mathrm{d} s \mathrm{~d} v \mathrm{~d} x \\
& +\int_{0}^{T} \int_{\gamma_{+}} \int_{-\min \left\{t, t_{\mathbf{b}}(t, x, v)\right\}}^{0} h(t+s, X(t+s ; t, x, v), V(t+s ; t, x, v)) \mathrm{d} s \mathrm{~d} \gamma \mathrm{d} t .
\end{aligned}
$$

Proof. The region $\left\{(t, x, v) \in[0, T] \times \Omega \times \mathbb{R}^{3}\right\}$ is a disjoint union of

$$
\begin{aligned}
& A:=\left\{(t, x, v) \in[0, T] \times \Omega \times \mathbb{R}^{3}: t_{\mathbf{f}}(t, x, v)+t \leq T\right\}, \\
& B:=\left\{(t, x, v) \in[0, T] \times \Omega \times \mathbb{R}^{3}: t_{\mathbf{f}}(t, x, v)+t>T\right\} .
\end{aligned}
$$

We also define

$$
\begin{aligned}
& A^{\prime}:=\left\{(t, s, x, v) \in[0, T] \times \mathbb{R} \times \gamma_{+}:-\min \left\{t_{\mathbf{b}}(t, x, v), t\right\} \leq s \leq 0\right\}, \\
& B^{\prime}:=\left\{(s, x, v) \in[0, T] \times \Omega \times \mathbb{R}^{3}: s \leq t_{\mathbf{b}}(T, x, v)\right\} .
\end{aligned}
$$

Let us denote (2.35) by $\mathcal{A}_{+}: A^{\prime} \rightarrow A$. Since $t_{\mathbf{f}}(t+s, X(t+s ; t, x, v), V(t+s ; t, x, v))+(t+s)=-s+(t+s)=$ $t \leq T$ if $(x, v) \in \gamma_{+}$and $-\min \left\{t_{\mathbf{b}}(t, x, v), t\right\}<s<0$, this map is well-defined. For any $(t, x, v) \in A$, we have

$$
\left(t+t_{\mathbf{f}}(t, x, v),-t_{\mathbf{f}}(t, x, v), X\left(t+t_{\mathbf{f}}(t, x, v) ; t, x, v\right), V\left(t+t_{\mathbf{f}}(t, x, v) ; t, x, v\right)\right) \in A^{\prime},
$$

since $t+t_{\mathbf{f}} \leq T$ and $t_{\mathbf{b}}\left(t+t_{\mathbf{f}}, X\left(t+t_{\mathbf{f}} ; t, x, v\right), V\left(t+t_{\mathbf{f}} ; t, x, v\right)\right)>t_{\mathbf{f}}$. Moreover

$$
\mathcal{A}_{+}\left(t+t_{\mathbf{f}},-t_{\mathbf{f}}, X\left(t+t_{\mathbf{f}} ; t, x, v\right), V\left(t+t_{\mathbf{f}} ; t, x, v\right)\right)=(t, x, v)
$$

implies that $\mathcal{A}_{+}$is surjective. From Lemma $3, \mathcal{A}_{+}$is bijective. Applying the change of variable $\mathcal{A}_{+}$with the Jacobian (2.37),

$$
\begin{aligned}
& \iiint_{A} h(t, x, v) \mathrm{d} t \mathrm{~d} x \mathrm{~d} v \\
= & \int_{0}^{T} \int_{\gamma_{+}} \int_{-\min \left\{t_{\mathbf{b}}(t, x, v), t\right\}}^{0} h(t+s, X(t+s ; t, x, v), V(t+s ; t, x, v) \mathrm{d} s \mathrm{~d} \gamma \mathrm{d} t .
\end{aligned}
$$

Next, we consider a map

$$
(s, x, v) \in B^{\prime} \mapsto(T-s, X(T-s ; T, x, v), V(T-s ; T, x, v)) \in B .
$$

From $t_{\mathbf{f}}(T-s, X(T-s ; T, x, v), V(T-s ; T, x, v))+(T-s)>s+(T-s)=T$, the map is well-defined. Since the characteristic is deterministic, the map is injective. Moreover it is also surjective since for $(s, x, v) \in B$ we have $(T-s, X(T ; s, x, v), V(T ; s, x, v)) \in B^{\prime}$ and $(T-s, X(T ; s, x, v), V(T ; s, x, v)) \mapsto(s, x, v)$ by this map (2.42). It is well-known that this map has a unit Jacobian (Liouville theorem). By the change of variable of (2.42) and $s \mapsto-s$

$$
\begin{aligned}
& \iiint_{B} h(t, x, v) \mathrm{d} t \mathrm{~d} x \mathrm{~d} v \\
= & \iint_{\Omega \times \mathbb{R}^{3}} \int_{-\min \left(T, t_{\mathbf{b}}(T, x, v)\right)}^{0} h(T+s, X(T+s ; T, x, v), V(T+s ; T, x, v) \mathrm{d} s \mathrm{~d} x \mathrm{~d} v .
\end{aligned}
$$

The first result is an "energy estimate" to the transport operator.

Lemma 6 (Green's identity). For $p \in[1, \infty)$, we assume $f \in L_{l o c}^{p}\left(\mathbb{R}_{+} \times \Omega \times \mathbb{R}^{3}\right)$ satisfies

$$
\partial_{t} f+v \cdot \nabla_{x} f+E \cdot \nabla_{v} f \in L_{l o c}^{p}\left(\mathbb{R}_{+} ; L^{p}\left(\Omega \times \mathbb{R}^{3}\right)\right), \quad f \in L_{l o c}^{p}\left(\mathbb{R}_{+} ; L^{p}\left(\gamma_{+}\right)\right) .
$$

Then $f \in C_{\text {loc }}^{0}\left(\mathbb{R}_{+} ; L^{p}\left(\Omega \times \mathbb{R}^{3}\right)\right)$ and $f \in L_{\text {loc }}^{p}\left(\mathbb{R}_{+} ; L^{p}\left(\gamma_{-}\right)\right)$. 
Moreover

$$
\begin{aligned}
\|f(T)\|_{p}^{p}+\int_{0}^{T}|f|_{p,+}^{p} & =\|f(0)\|_{p}^{p}+\int_{0}^{T}|f|_{p,-}^{p} \\
& +p \int_{0}^{T} \iint_{\Omega \times \mathbb{R}^{3}}\left\{\partial_{t}+v \cdot \nabla_{x} f+E \cdot \nabla_{v} f\right\}|f|^{p-2} f .
\end{aligned}
$$

Proof. By Lemma 5 ,

$$
\begin{aligned}
& \quad p \int_{0}^{T} \iint_{\Omega \times \mathbb{R}^{3}}\left\{\partial_{t} f+v \cdot \nabla_{x} f+E \cdot \nabla_{v} f\right\}|f|^{p-2} f \mathrm{~d} x \mathrm{~d} v \mathrm{~d} t \\
& =p \iint_{\Omega \times \mathbb{R}^{3}} \int_{-\min \left\{T, t_{\mathbf{b}}(T, x, v)\right\}}\left\{\partial_{t} f+v \cdot \nabla_{x} f+E \cdot \nabla_{v} f\right\} \\
& \quad \times|f|^{p-2} f(T+s, X(T+s ; T, x, v), V(T+s ; T, x, v)) \mathrm{d} s \mathrm{~d} v \mathrm{~d} x \\
& +p \int_{0}^{T} \int_{\gamma_{+}} \int_{-\min \left\{t, t_{\mathbf{b}}(t, x, v)\right\}}^{0}\left\{\partial_{t} f+v \cdot \nabla_{x} f+E \cdot \nabla_{v} f\right\} \\
& \quad \times|f|^{p-2} f(t+s, X(t+s ; t, x, v), V(t+s ; t, x, v)) \mathrm{d} s \mathrm{~d} \gamma \mathrm{d} t .
\end{aligned}
$$

Note that

$$
\begin{aligned}
& \frac{d}{d s}|f(t+s, X(t+s ; t, x, v), V(t+s ; t, x, v))|^{p} \\
= & \left\{\partial_{t} f+v \cdot \nabla_{x} f+E \cdot \nabla_{v} f\right\} \\
& \times|f|^{p-2} f(t+s, X(t+s ; t, x, v), V(t+s ; t, x, v)) .
\end{aligned}
$$

We plug this into (2.44) and apply the integration by parts in $s$ to obtain

$$
\begin{aligned}
& \frac{1}{p} \times(2.44)=\iint_{\Omega \times \mathbb{R}^{3}}|f(T, x, v)|^{p} \mathrm{~d} x \mathrm{~d} v+\int_{0}^{T} \int_{\gamma^{+}}|f(t, x, v)|^{p} \mathrm{~d} \gamma \mathrm{d} t \\
& -\underbrace{\iint_{\Omega \times \mathbb{R}^{3}} \mathbf{1}_{T \geq t_{\mathbf{b}}(T, x, v)}\left|f\left(T-t_{\mathbf{b}}, x_{\mathbf{b}}, v_{\mathbf{b}}\right)\right|^{p} \mathrm{~d} x \mathrm{~d} v}_{2.45} \\
& -\underbrace{\iint_{\Omega \times \mathbb{R}^{3}} \mathbf{1}_{T<t_{\mathbf{b}}(T, x, v)}|f(0, X(0 ; T, x, v), V(0 ; T, x, v))|^{p} \mathrm{~d} x \mathrm{~d} v}_{\text {2.45 }_{2}} \\
& -\underbrace{\int_{0}^{T} \int_{\gamma_{+}} \mathbf{1}_{t \geq t_{\mathbf{b}}(t, x, v)}\left|f\left(t-t_{\mathbf{b}}, x_{\mathbf{b}}, v_{\mathbf{b}}\right)\right|^{p} \mathrm{~d} \gamma \mathrm{d} t} \\
& 2_{2.45}{ }_{3} \\
& -\underbrace{\int_{0}^{T} \int_{\gamma_{+}} \mathbf{1}_{t<t_{\mathbf{b}}(t, x, v)}|f(0, X(0 ; t, x, v), V(0 ; t, x, v))|^{p} \mathrm{~d} \gamma \mathrm{d} t} . \\
& 2^{2.45} 4
\end{aligned}
$$

We claim that

$$
\left.(2.45)_{2}+2.45\right)_{4}=\iint_{\Omega \times \mathbb{R}^{3}}\left|f_{0}\right|^{p} \mathrm{~d} x \mathrm{~d} v .
$$

If $T<t_{\mathbf{b}}(T, x, v)$ then $t_{\mathbf{f}}(0, X(0 ; T, x, v), V(0 ; T, x, v))>T$. On the other hand, if $t<t_{\mathbf{b}}(t, x, v)$ then $t_{\mathbf{f}}(0, X(0 ; t, x, v), V(0 ; t, x, v))<T$. Now we apply the change of variables $(x, v) \mapsto(X(0 ; t, x, v), V(0 ; t, x, v))$ to $(2.45)_{2}$ and to $(2.45)_{4}$ to conclude (2.46).

Next we claim that

$$
(2.45)_{1}+(\underline{2.45})_{3}=\int_{0}^{T}|f|_{p,-}^{p} .
$$

We split

$$
[0, T] \times \gamma_{-}=\left\{t_{\mathbf{f}}(s, x, v)+s>T\right\} \cup\left\{t_{\mathbf{f}}(s, x, v)+s \leq T\right\} .
$$


We consider the map (2.20) for fixed $T>0$ from $\Omega \times \mathbb{R}^{3}$ to $\left\{(s, x, v) \in[0, T] \times \gamma_{-}: t_{\mathbf{f}}(s, x, v)+s>T\right\}$. This map is onto since $t_{\mathbf{b}}(T, X(T ; s, x, v), V(T ; s, x, v))=T-s$ and therefore $(X(T ; s, x, v), V(T ; s, x, v)) \mapsto(s, x, v)$. By the change of variables with (2.21)

$$
\text { (2.45) } 1=\int_{0}^{T} \iint_{\gamma_{-}} \mathbf{1}_{t_{\mathbf{f}}(s, x, v)+s>T}|f(s, x, v)|^{p} \mathrm{~d} \gamma \mathrm{d} s .
$$

For (2.45) 3 we consider (2.22) from $[0, T] \times \gamma_{+}$to $\left\{(s, x, v) \in[0, T] \times \gamma_{-}: t_{\mathbf{f}}(s, x, v)+s \leq T\right\}$. This map is onto since $t_{\mathbf{b}}\left(s+t_{\mathbf{f}}(s, x, v), X\left(s+t_{\mathbf{f}} ; s, x, v\right), V\left(s+t_{\mathbf{f}} ; s, x, v\right)\right)=t_{\mathbf{f}}(s, x, v)$ and therefore $\left(s+t_{\mathbf{f}}(s, x, v), X(s+\right.$ $\left.\left.t_{\mathbf{f}} ; s, x, v\right), V\left(s+t_{\mathbf{f}} ; s, x, v\right)\right) \mapsto(s, x, v)$. Applying the change of variables of (2.23), we obtain that

$$
(2.45)_{2}=\int_{0}^{T} \iint_{\gamma_{-}} \mathbf{1}_{t_{\mathbf{f}}(s, x, v)+s \leq T}|f(s, x, v)|^{p} \mathrm{~d} \gamma \mathrm{d} s .
$$

We define

$$
\gamma_{+}^{\varepsilon}:=\left\{(x, v) \in \gamma_{+}:|n(x) \cdot v| \leq \varepsilon \text { or }|v| \geq 1 / \varepsilon\right\} .
$$

Lemma 7. Assume that, for $\Lambda_{1}>0, \delta_{1}>0$,

$$
\sup _{t \geq 0} e^{\Lambda_{1} t}\|E(t)\|_{\infty} \leq \delta_{1} \ll 1 .
$$

We also assume $\frac{1}{C}\langle v\rangle \leq \psi(t, x, v) \leq C\langle v\rangle$ for some $C>0$. For $\varepsilon$ satisfying

$$
\varepsilon>\frac{2 \delta_{1}}{\Lambda_{1}}>0
$$

there exists a constant $C_{\delta_{1}, \Lambda_{1}, \Omega}>0$ for all $t \geq 0$, such that

$$
\begin{aligned}
& \int_{0}^{t} \int_{\gamma_{+} \backslash \gamma_{+}^{\varepsilon}}|h| \mathrm{d} \gamma \mathrm{d} s \\
& \leq C_{\delta_{1}, \Lambda_{1}, \Omega}\left\{\left\|h_{0}\right\|_{1}+\int_{0}^{t}\|h(s)\|_{1} \mathrm{~d} s\right. \\
& \left.\quad+\int_{0}^{t}\left\|\left[\partial_{t}+v \cdot \nabla_{x}+E \cdot \nabla_{v}+\psi\right] h(s)\right\|_{1} \mathrm{~d} s\right\} .
\end{aligned}
$$

If $E \in L^{\infty}$ does not decay but

$$
\|E(t)\|_{\infty} \leq \delta
$$

then for $\varepsilon>0$,

$$
\begin{aligned}
& \int_{0}^{t} \int_{\gamma_{+} \backslash \gamma_{+}^{\varepsilon}}|h| \mathrm{d} \gamma \mathrm{d} s \\
& \leq C_{\delta, t, \varepsilon, \Omega}\left\{\left\|h_{0}\right\|_{1}+\int_{0}^{t}\|h(s)\|_{1} \mathrm{~d} s\right. \\
& \left.\quad+\int_{0}^{t}\left\|\left[\partial_{t}+v \cdot \nabla_{x}+E \cdot \nabla_{v}+\psi\right] h(s)\right\|_{1} \mathrm{~d} s\right\},
\end{aligned}
$$

where we have time-dependent constant $C_{\delta, t, \varepsilon, \Omega}>0$.

Proof. For $t-t_{\mathbf{b}}(t, x, v) \leq s \leq t$, from (2.50),

$$
|V(s ; t, x, v)-v| \leq \int_{s}^{t}|E(\tau, X(\tau ; t, x, v))| \mathrm{d} \tau \leq \frac{\delta_{1}}{\Lambda_{1}} .
$$

Therefore, for $|v| \geq \varepsilon$ with the condition (2.51),

$$
\begin{aligned}
\left|X\left(t-t_{\mathbf{b}} ; t, x, v\right)-x\right| & \geq\left|\int_{t-t_{\mathbf{b}}}^{t} v\right|-\int_{t-t_{\mathbf{b}}}^{t}|V(\tau ; t, x, v)-v| \mathrm{d} \tau \\
& \geq|v| t_{\mathbf{b}}(t, x, v)-\frac{\delta_{1}}{\Lambda_{1}} t_{\mathbf{b}}(t, x, v) \geq \frac{\delta_{1}}{\Lambda_{1}} t_{\mathbf{b}}(t, x, v) .
\end{aligned}
$$


This gives an upper bound as

$$
t_{\mathbf{b}}(t, x, v) \leq \frac{\Lambda_{1}}{\delta_{1}} \times \operatorname{diam}(\Omega) \text { for }|v| \geq \varepsilon .
$$

Now we consider a lower bound of $t_{\mathbf{b}}(t, x, v)$ for $(t, x, v) \in[0, \infty) \times \gamma_{+} \backslash \gamma_{+}^{\varepsilon}$. Since

$$
\lim _{\substack{y \rightarrow x_{1} \\ y \in \partial \Omega}} \frac{\left|\left(x_{1}-y\right) \cdot n\left(x_{1}\right)\right|}{\left|x_{1}-y\right|}=0, \quad \text { for } \quad x_{1} \in \partial \Omega,
$$

we have $\left|\left(x_{1}-y\right) \cdot n\left(x_{1}\right)\right| \leq C_{\Omega}\left|x_{1}-y\right|^{2}$ for all $x_{1}, y \in \partial \Omega$. Thus from (2.55),

$$
\begin{aligned}
C_{\Omega}\left|x-x_{\mathbf{b}}\right|^{2} & \geq\left|\left(x_{\mathbf{b}}-x\right) \cdot n(x)\right|=\left|\int_{t-t_{\mathbf{b}}}^{t} V(\tau ; t, x, v) \mathrm{d} \tau \cdot n(x)\right| \\
& \geq\left|\int_{t-t_{\mathbf{b}}}^{t} v \cdot n(x) \mathrm{d} \tau\right|-\int_{t-t_{\mathbf{b}}}^{t}|v-V(\tau ; t, x, v)| \mathrm{d} \tau \\
& \geq\left\{|v \cdot n(x)|-\frac{\delta_{1}}{\Lambda_{1}}\right\} t_{\mathbf{b}}>\frac{\varepsilon}{2} t_{\mathbf{b}} .
\end{aligned}
$$

Also we have

$$
\begin{aligned}
\left|x-x_{\mathbf{b}}\right|^{2} & =\left|t_{\mathbf{b}} v-\int_{t-t_{\mathbf{b}}}^{t}(v-V(s)) \mathrm{d} s\right|^{2} \\
& \leq 2 t_{\mathbf{b}}^{2}|v|^{2}+2\left(\int_{t-t_{\mathbf{b}}}^{t}|v-V(s)| \mathrm{d} s\right)^{2} \\
& \leq 2 t_{\mathbf{b}}^{2}|v|^{2}+2\left(t_{\mathbf{b}} \frac{\delta_{1}}{\Lambda_{1}}\right)^{2}<2 t_{\mathbf{b}}^{2}|v|^{2}+\frac{1}{2} t_{\mathbf{b}}^{2} \varepsilon^{2} .
\end{aligned}
$$

Combining above two estimates, we get $C_{\Omega}\left(2 t_{\mathbf{b}}^{2}|v|^{2}+\frac{1}{2} t_{\mathbf{b}}^{2} \varepsilon^{2}\right)>\frac{\varepsilon}{2} t_{\mathbf{b}}$, and dividing $|v|^{2} t_{\mathbf{b}}$ on both sides we get

$$
t_{\mathbf{b}}(t, x, v)>\frac{1}{5 C_{\Omega}} \varepsilon^{3} .
$$

If $h$ solves (2.6), then for $(t, x, v) \in[0, T] \times \gamma_{+}$and $-\min \left\{t, t_{\mathbf{b}}(t, x, v)\right\} \leq s \leq 0$,

$$
\begin{aligned}
h(t, x, v) & =h(t+s, X(t+s), V(t+s)) e^{-\int_{t-s}^{t} \psi\left(\tau^{\prime}, X\left(\tau^{\prime}\right), V\left(\tau^{\prime}\right)\right) \mathrm{d} \tau^{\prime}} \\
& +\int_{t+s}^{t} e^{-\int_{\tau}^{t} \psi\left(\tau^{\prime}, X\left(\tau^{\prime}\right), V\left(\tau^{\prime}\right)\right) \mathrm{d} \tau^{\prime}} H(\tau, X(\tau ; t, x, v), V(\tau ; t, x, v)) \mathrm{d} \tau,
\end{aligned}
$$

where $X(s)=X(s ; t, x, v), V(s)=V(s ; t, x, v)$.

Then

$$
\begin{aligned}
& \min \left\{t, t_{\mathbf{b}}(t, x, v)\right\} \times|h(t, x, v)| \\
= & \int_{-\min \left\{t, t_{\mathbf{b}}(t, x, v)\right\}}^{0}|h(t, x, v)| \mathrm{d} s \\
\leq & \int_{-\min \left\{t, t_{\mathbf{b}}(t, x, v)\right\}}^{0}|h(t+s, X(t+s), V(t+s))| \mathrm{d} s \\
& +\int_{-\min \left\{t, t_{\mathbf{b}}(t, x, v)\right\}}^{0} \int_{t+s}^{t}|H(\tau, X(\tau ; t, x, v), V(\tau ; t, x, v))| \mathrm{d} \tau \mathrm{d} s \\
\leq & \int_{-\min \left\{t, t_{\mathbf{b}}(t, x, v)\right\}}^{0}|h(t+s, X(t+s), V(t+s))| \mathrm{d} s \\
& +t_{\mathbf{b}}(t, x, v) \int_{t-\min \left\{t, t_{\mathbf{b}}(t, x, v)\right\}}^{t}|H(\tau, X(\tau ; t, x, v), V(\tau ; t, x, v))| \mathrm{d} \tau .
\end{aligned}
$$


Let $\epsilon_{1}:=\frac{1}{5 C_{\Omega}} \varepsilon^{3}$. Then from (2.59), for $(t, x, v) \in\left[\epsilon_{1}, T\right] \times \gamma_{+} \backslash \gamma_{+}^{\varepsilon}$, we integrate over $\int_{\epsilon_{1}}^{T} \int_{\gamma_{+} \backslash \gamma_{+}^{\varepsilon}}$ to get

$$
\begin{aligned}
& \epsilon_{1} \int_{\epsilon_{1}}^{T} \int_{\gamma_{+} \backslash \gamma_{+}^{\varepsilon}}|h(t, x, v)| \mathrm{d} \gamma \mathrm{d} t \\
\leq & \int_{\epsilon_{1}}^{T} \int_{\gamma_{+} \backslash \gamma_{+}^{\varepsilon}} \min _{\left[\epsilon_{1}, T\right] \times\left[\gamma_{+} \backslash \gamma_{+}^{\varepsilon}\right]}\left\{t, t_{\mathbf{b}}(t, x, v)\right\} \times|h(t, x, v)| \mathrm{d} \gamma \mathrm{d} t \\
\leq & \int_{0}^{T} \int_{\gamma_{+} \backslash \gamma_{+}^{\varepsilon}} \int_{-\min \left\{t, t_{\mathbf{b}}(t, x, v)\right\}}^{0}|h(t+s, X(t+s), V(t+s))| \mathrm{d} s \mathrm{~d} \gamma \mathrm{d} t \\
& +\sup _{\gamma+\backslash \gamma_{+}^{\varepsilon}} t_{\mathbf{b}}(t, x, v) \int_{0}^{T} \int_{\gamma_{+} \backslash \gamma_{+}^{\varepsilon}} \int_{t-\min \left\{t, t_{\mathbf{b}}(t, x, v)\right\}}^{t}|H(\tau, X(\tau), V(\tau))| \\
\lesssim & \int_{0}^{T}\|h\|_{1}+\int_{0}^{T}\left\|\left[\partial_{t}+v \cdot \nabla_{x}-\nabla \phi \cdot \nabla_{v}+\psi\right] h\right\|_{1},
\end{aligned}
$$

where we used Lemma 5 and (2.56) in the last inequality.

On the other hand, from our choice $\varepsilon$ and $\epsilon_{1}$,

$$
t_{\mathbf{b}}(t, x, v)>t \text { for all }(t, x, v) \in\left[0, \epsilon_{1}\right] \times \gamma_{+} \backslash \gamma_{+}^{\varepsilon} .
$$

Integrating

$$
|h(t, x, v)| \leq\left|h_{0}(X(0), V(0))\right|+\int_{-t}^{0}|H(t+\tau, X(t+\tau), V(t+\tau))| \mathrm{d} \tau
$$

over $\int_{0}^{\epsilon_{1}} \int_{\gamma_{+} \backslash \gamma_{+}^{\varepsilon}}$, we get

$$
\begin{aligned}
& \int_{0}^{\epsilon_{1}} \int_{\gamma_{+} \backslash \gamma_{+}^{\varepsilon}}|h(t, x, v)| \\
= & \int_{0}^{\epsilon_{1}} \int_{\gamma_{+} \backslash \gamma_{+}^{\varepsilon}}\left|h_{0}(X(0), V(0))\right| \mathrm{d} \gamma \mathrm{d} t \\
& +\int_{0}^{\epsilon_{1}} \int_{\gamma_{+} \backslash \gamma_{+}^{\varepsilon}} \int_{-t}^{0}|H(t+\tau, X(t+\tau), V(t+\tau))| \mathrm{d} \tau \mathrm{d} \gamma \mathrm{d} t .
\end{aligned}
$$

Applying the change of variables of (2.35), the first term in RHS of (2.64) is bounded by $\left\|h_{0}\right\|_{1}$. From (2.63) and (2.35), the second term in RHS is bounded by

$$
\int_{0}^{\epsilon_{1}}\left\|\left(\partial_{t}+v \cdot \nabla_{x}-\nabla \phi \cdot \nabla_{v}+\psi\right) h\right\|_{1} \mathrm{~d} t .
$$

Finally we combine (2.64) and (2.62) to obtain (2.52).

The proof of (2.54) is similar. In the last line of (2.61), we have $t$ multiplier instead of $t_{\mathbf{b}}(t, x, v)$. The rest of proof is same.

Lemma 8. If $n\left(x_{\mathbf{b}}(t, x, v)\right) \cdot v_{\mathbf{b}}(t, x, v) \neq 0$ then $\left(t_{\mathbf{b}}, x_{\mathbf{b}}, v_{\mathbf{b}}\right)$ is differentiable and

$$
\begin{aligned}
\frac{\partial t_{\mathbf{b}}}{\partial x_{i}} & =\frac{1}{n\left(x_{\mathbf{b}}\right) \cdot v_{\mathbf{b}}} n\left(x_{\mathbf{b}}\right) \cdot\left[e_{i}+\int_{t}^{t-t_{\mathbf{b}}} \int_{t}^{s}\left(\frac{\partial X(\tau)}{\partial x_{i}} \cdot \nabla\right) E(\tau, X(\tau)) \mathrm{d} \tau \mathrm{d} s\right], \\
\frac{\partial x_{\mathbf{b}}}{\partial x_{i}} & =e_{i}-\frac{\partial t_{\mathbf{b}}}{\partial x_{i}} v_{\mathbf{b}}+\int_{t}^{t-t_{\mathbf{b}}} \int_{t}^{s}\left(\frac{\partial X(\tau)}{\partial x_{i}} \cdot \nabla\right) E(\tau, X(\tau)) \mathrm{d} \tau \mathrm{d} s, \\
\frac{\partial v_{\mathbf{b}}}{\partial x_{i}} & =-\frac{\partial t_{\mathbf{b}}}{\partial x_{i}} E\left(t-t_{\mathbf{b}}, x_{\mathbf{b}}\right)+\int_{t}^{t-t_{\mathbf{b}}}\left(\frac{\partial X(\tau)}{\partial x_{i}} \cdot \nabla\right) E(\tau, X(\tau)) \mathrm{d} \tau, \\
\frac{\partial t_{\mathbf{b}}}{\partial v_{i}} & =\frac{1}{n\left(x_{\mathbf{b}}\right) \cdot v_{\mathbf{b}}} n\left(x_{\mathbf{b}}\right) \cdot\left[e_{i}+\int_{t}^{t-t_{\mathbf{b}}} \int_{t}^{s}\left(\frac{\partial X(\tau)}{\partial v_{i}} \cdot \nabla\right) E(\tau, X(\tau)) \mathrm{d} \tau \mathrm{d} s\right], \\
\frac{\partial x_{\mathbf{b}}}{\partial v_{i}} & =-t_{\mathbf{b}} e_{i}-\frac{\partial t_{\mathbf{b}}}{\partial v_{i}} v_{\mathbf{b}}+\int_{t}^{t-t_{\mathbf{b}}} \int_{t}^{s}\left(\frac{\partial X(\tau)}{\partial v_{i}} \cdot \nabla\right) E(\tau, X(\tau)) \mathrm{d} \tau \mathrm{d} s, \\
\frac{\partial v_{\mathbf{b}}}{\partial v_{i}} & =e_{i}-\frac{\partial t_{\mathbf{b}}}{\partial v_{i}} E\left(t-t_{\mathbf{b}}, x_{\mathbf{b}}\right)+\int_{t}^{t-t_{\mathbf{b}}}\left(\frac{\partial X(\tau)}{\partial v_{i}} \cdot \nabla\right) E(\tau, X(\tau)) \mathrm{d} \tau .
\end{aligned}
$$


Proof. The equalities are derived from direct computations and an implicit function theorem. For details see [27.

Proposition 2. Assume the compatibility condition

$$
f_{0}(x, v)=g(0, x, v) \quad \text { for } \quad(x, v) \in \gamma_{-} .
$$

Let $p \in[1, \infty)$ and $0<\vartheta<1 / 4$. Assume

$$
\begin{array}{r}
\nabla_{x} f_{0}, \nabla_{v} f_{0} \in L^{p}\left(\Omega \times \mathbb{R}^{3}\right), \\
\nabla_{x, v} t_{\mathbf{b}} \partial_{t} g, \nabla_{x, v} v_{\mathbf{b}} \nabla_{v} g, \nabla_{x, v} x_{\mathbf{b}} \partial_{x_{\mathbf{b}}} g, \nabla_{x, v} t_{\mathbf{b}} \psi g \in L^{p}\left([0, T] \times \gamma_{-}\right), \\
\nabla_{x} H, \nabla_{v} H \in L^{p}\left([0, T] \times \Omega \times \mathbb{R}^{3}\right), \\
e^{-\vartheta|v|^{2}} \nabla_{x} \psi, e^{-\vartheta|v|^{2}} \nabla_{v} \psi \in L^{p}\left([0, T] \times \Omega \times \mathbb{R}^{3}\right), \\
e^{\vartheta|v|^{2}} f_{0} \in L^{\infty}\left(\Omega \times \mathbb{R}^{3}\right), e^{\vartheta|v|^{2}} g \in L^{\infty}\left([0, T] \times \gamma_{-}\right), \\
e^{\vartheta|v|^{2}} H \in L^{\infty}\left([0, T] \times \Omega \times \mathbb{R}^{3}\right) .
\end{array}
$$

Then for any $T>0$, there exists a unique solution $f$ to (2.6) such that $\nabla_{x, v} f \in C^{0}\left([0, T] ; L^{p}\left(\Omega \times \mathbb{R}^{3}\right)\right) \cap$ $L^{1}\left((0, T) ; L^{p}(\gamma)\right)$.

Proof. Along the characteristics

$$
\begin{aligned}
f(t, x, v) & =\mathbf{1}_{t<t_{\mathbf{b}}} e^{-\int_{0}^{t} \psi(\tau, X(\tau), V(\tau)) \mathrm{d} \tau} f_{0}(X(0), V(0)) \\
& +\mathbf{1}_{t<t_{\mathbf{b}}} \int_{0}^{t} e^{-\int_{s}^{t} \psi(\tau, X(\tau), V(\tau)) \mathrm{d} \tau} H(s, X(s), V(s)) \mathrm{d} s \\
& +\mathbf{1}_{t>t_{\mathbf{b}}} e^{-\int_{t-t_{\mathbf{b}}}^{t} \psi(\tau, X(\tau), V(\tau)) \mathrm{d} \tau} g\left(t-t_{\mathbf{b}}, X\left(t-t_{\mathbf{b}}\right), V\left(t-t_{\mathbf{b}}\right)\right) \\
& +\mathbf{1}_{t>t_{\mathbf{b}}} \int_{t-t_{\mathbf{b}}}^{t} e^{-\int_{s}^{t} \psi(\tau, X(\tau), V(\tau)) \mathrm{d} \tau} H(s, X(s), V(s)) \mathrm{d} s,
\end{aligned}
$$

where $(X(s), V(s))=(X(s ; t, x, v), V(s ; t, x, v))$.

By direct computations, we have

$$
\begin{gathered}
\nabla_{x, v} f(t, x, v) \mathbf{1}_{\left\{t \neq t_{\mathbf{b}}\right\}} \\
=\mathbf{1}_{\left\{t<t_{\mathbf{b}}\right\}} e^{-\int_{0}^{t} \psi}\left\{\nabla_{x, v} X(0) \cdot \nabla_{x} f_{0}+\nabla_{x, v} V(0) \cdot \nabla_{v} f_{0}\right. \\
\left.\quad-f_{0} \int_{0}^{t}\left(\nabla_{x, v} X(0) \cdot \nabla_{x} \psi+\nabla_{x, v} V(0) \cdot \nabla_{v} \psi\right)\right\} \\
+\mathbf{1}_{\left\{t>t_{\mathbf{b}}\right\}} e^{-\int_{t-t_{\mathbf{b}}}^{t} \psi}\left\{-\nabla_{x, v} t_{\mathbf{b}} \partial_{t} g\left(t-t_{\mathbf{b}}\right)+\nabla_{x, v} x_{\mathbf{b}} \cdot \nabla_{x} g\left(t-t_{\mathbf{b}}\right)\right. \\
+\nabla_{x, v} v_{\mathbf{b}} \cdot \nabla_{v} g\left(t-t_{\mathbf{b}}\right)-\nabla_{x, v} t_{\mathbf{b}} \psi\left(t-t_{\mathbf{b}}\right) g\left(t-t_{\mathbf{b}}\right) \\
\left.-g\left(t-t_{\mathbf{b}}\right) \int_{t-t_{\mathbf{b}}}^{t}\left(\nabla_{x, v} X(\tau) \cdot \nabla_{x} \psi(\tau)+\nabla_{x, v} V(\tau) \cdot \nabla_{v} \psi(\tau)\right) \mathrm{d} \tau\right\} \\
+\int_{\max \left\{0, t-t_{\mathbf{b}}\right\}}^{t} e^{-\int_{s}^{t} \psi}\left\{\nabla_{x, v} X(s) \cdot \nabla_{x} H(s)+\nabla_{x, v} V(s) \cdot \nabla_{v} H(s)\right. \\
\left.-H(s) \int_{s}^{t}\left(\nabla_{x, v} X(\tau) \cdot \nabla_{x} \psi(\tau)+\nabla_{x, v} V(\tau) \cdot \nabla_{v} \psi(\tau)\right) \mathrm{d} \tau\right\} \mathrm{d} s,
\end{gathered}
$$

where $\nabla_{x, v} t_{\mathbf{b}}, \nabla_{x, v} x_{\mathbf{b}}, \nabla_{x, v} v_{\mathbf{b}}$ in (2.65).

From (1.22) with replacing $-\nabla_{x} \phi_{f}$ by $E$,

$$
\begin{aligned}
& \frac{d}{d s}\left[\begin{array}{cc}
\nabla_{x, v} X(s ; t, x, v) \\
\nabla_{x, v} V(s ; t, x, v)
\end{array}\right] \\
= & {\left[\begin{array}{cc}
0_{3 \times 3} & \operatorname{Id}_{3 \times 3} \\
\nabla_{x} E(s, X(s ; t, x, v)) & 0_{3 \times 3}
\end{array}\right]\left[\begin{array}{l}
\nabla_{x, v} X(s ; t, x, v) \\
\nabla_{x, v} V(s ; t, x, v)
\end{array}\right] . }
\end{aligned}
$$

Then easily we have for $C=C\left(\left\|\nabla_{x} E\right\|_{\infty}^{1 / 2}\right)$

$$
\left|\nabla_{x, v} X(s ; t, x, v)\right|+\left|\nabla_{x, v} V(s ; t, x, v)\right| \lesssim e^{C|t-s|} .
$$


By the change of variables in Lemma 3 and Lemma 5 , we have

$$
\begin{aligned}
& \left\|\nabla_{x, v} f(t) \mathbf{1}_{t \neq t_{\mathbf{b}}}\right\|_{p} \\
& \lesssim_{t}\left\|\nabla_{x, v} f_{0}\right\|_{p}+\left\|e^{\vartheta|v|^{2}} f_{0}\right\|_{\infty}\left\|e^{-\vartheta|v|^{2}} \nabla_{x, v} \psi\right\|_{p} \\
& +\left[\int_{0}^{t}\left\|\nabla_{x, v} t_{\mathbf{b}} \partial_{t} g\right\|_{p}^{p}+\left\|\nabla_{x, v} x_{\mathbf{b}} \partial_{x} g\right\|_{p}^{p}\right. \\
& \left.+\left\|\nabla_{x, v} v_{\mathbf{b}} \partial_{v} g\right\|_{p}^{p}+\left\|\nabla_{x, v} t_{\mathbf{b}} \psi g\right\|_{p}^{p}\right]^{1 / p} \\
& +\left[\int_{0}^{t}\left\|\nabla_{x, v} H\right\|_{p}^{p}+\left\|e^{\vartheta|v|^{2}} H\right\|_{\infty}\left\|e^{-\vartheta|v|^{2}} \nabla_{x, v} \psi\right\|_{p}^{p}\right]^{1 / p} .
\end{aligned}
$$

From our hypothesis (2.67), these terms are bounded and therefore,

$$
\nabla_{x} f \mathbf{1}_{\left\{t \neq t_{\mathbf{b}}\right\}}, \nabla_{v} f \mathbf{1}_{\left\{t \neq t_{\mathbf{b}}\right\}} \in L^{\infty}\left([0, T] ; L^{p}\left(\Omega \times \mathbb{R}^{3}\right)\right) .
$$

Now we prove that $\nabla_{x, v} f \mathbf{1}_{t \neq t_{\mathbf{b}}}$ is weak derivatives of $f$. We claim the following: Let $\Phi(t, x, v) \in C_{c}^{\infty}((0, T) \times$ $\Omega \times \mathbb{R}^{3}$ ) then we have

$$
\int_{0}^{T} \iint_{\Omega \times \mathbb{R}^{3}} f \nabla_{x, v} \Phi=-\int_{0}^{T} \iint_{\Omega \times \mathbb{R}^{3}} \nabla_{x, v} f \mathbf{1}_{\left\{t \neq t_{\mathbf{b}}\right\}} \Phi .
$$

Fix the test function $\Phi(t, x, v)$. From Lemma 2, we have (2.8) for all $(t, x, v) \in \mathbb{R}+\times \Omega \times \mathbb{R}^{3}$. Since supp $(\Phi)$ is compact and $n\left(x_{\mathbf{b}}(t, x, v)\right) \cdot v_{\mathbf{b}}(t, x, v)$ is continuous if (2.8) we have

$$
\sup _{(t, x, v) \in \operatorname{supp}(\Phi)}\left|n\left(x_{\mathbf{b}}(t, x, v)\right) \cdot v_{\mathbf{b}}(t, x, v)\right|>\delta_{\Phi}>0 .
$$

Therefore, from (2.65),$t_{\mathbf{b}}(t, x, v)$ is differentiable in $\operatorname{supp}(\Phi)$ and hence

$$
\mathcal{M}:=\left\{\left(t_{\mathbf{b}}(t, x, v), x, v\right):(t, x, v) \in \operatorname{supp}(\Phi)\right\}
$$

is a $C^{1}$-manifold in $\mathbb{R}_{+} \times \Omega \times \mathbb{R}^{3}$ with the normal direction

$$
n_{\mathcal{M}}:=\frac{1}{\sqrt{1+\left|\nabla_{x} t_{\mathbf{b}}\right|^{2}+\left|\nabla_{v} t_{\mathbf{b}}\right|^{2}}}\left(1,-\nabla_{x} t_{\mathbf{b}},-\nabla_{v} t_{\mathbf{b}}\right) \in \mathbb{R}^{7} .
$$

Now we take $C^{1}$-approximation $\left(f_{0}^{l}, H^{l}, g^{l}\right)$ of $\left(f_{0}, H, g\right)$ such that, as $l \uparrow \infty$

$$
\begin{aligned}
& \left\|f_{0}^{l}-f_{0}\right\|_{W^{1, p}\left(\Omega \times B_{R}(0)\right)}+\left\|g^{l}-g\right\|_{W^{1, p}\left([0, T] \times\left\{\gamma_{-} \cap \partial \Omega \times B_{R}(0)\right\}\right)} \\
& \quad+\left\|H^{l}-H\right\|_{W^{1, p}\left([0, T] \times \Omega \times B_{R}(0)\right)} \rightarrow 0,
\end{aligned}
$$

where $B_{R}(0)$ includes projection (onto velocity phase) of $\operatorname{supp}(\Phi)$. Then by the trace theorem, for $0<\delta \ll 1$

$$
f_{0}^{l}(x, v) \rightarrow f_{0}(x, v) \text { and } g^{l}(0, x, v) \rightarrow g(0, x, v) \text { in } L^{1}\left(\gamma_{-} \backslash \gamma_{-}^{\delta}\right)
$$

We define

$$
\begin{aligned}
f^{l}(t, x, v)= & \mathbf{1}_{t<t_{\mathbf{b}}} e^{-\int_{0}^{t} \psi} f_{0}^{l}(X(0), V(0)) \\
& +\mathbf{1}_{t>t_{\mathbf{b}}} e^{-\int_{t-t_{\mathbf{b}}}^{t} \psi} g^{l}\left(t-t_{\mathbf{b}}, x_{\mathbf{b}}, v_{\mathbf{b}}\right) \\
& +\int_{\max \left\{t-t_{\mathbf{b}}, 0\right\}}^{t} e^{-\int_{s}^{t} \psi} H^{l}(s, X(s), V(s)) \mathrm{d} s
\end{aligned}
$$

and $f_{+}^{l}(t, x, v):=\mathbf{1}_{t>t_{\mathbf{b}}} f^{l}(t, x, v)$ and $f_{-}^{l}(t, x, v):=\mathbf{1}_{t<t_{\mathbf{b}}} f^{l}(t, x, v)$. We can derive the same estimate as (2.70). This implies that up to subsequence

$$
\nabla f_{ \pm}^{l} \rightarrow \nabla f_{ \pm} \text {weakly in } L^{p} .
$$

By the Gauss theorem, for a standard basis $e_{i} \in \mathbb{R}_{t} \times \mathbb{R}_{x}^{3} \times \mathbb{R}_{v}^{3}$ with $i=2,3, \cdots, 7$,

$$
\begin{aligned}
& \iiint_{i} e_{i} \cdot \nabla_{x, v} \Phi f^{l} \mathrm{~d} x \mathrm{~d} v \mathrm{~d} t \\
= & \iint\left[f_{+}^{l}-f_{-}^{l}\right] \Phi \mathbf{e} \cdot n_{\mathcal{M}} \mathrm{d} \mathcal{M} \\
- & \left\{\iiint_{t>t_{\mathbf{b}}} \Phi e_{i} \cdot \nabla_{x, v} f_{+}^{l}+\iiint_{t<t_{\mathbf{b}}} \Phi e_{i} \cdot \nabla_{x, v} f_{-}^{l}\right\} .
\end{aligned}
$$


From (2.75),

$$
\lim _{t \downarrow t_{\mathbf{b}}} f_{+}^{l}(t, x, v)=e^{-\int_{0}^{t} \psi} g^{l}\left(0, x_{\mathbf{b}}, v_{\mathbf{b}}\right)+\int_{0}^{t} e^{-\int_{s}^{t} \psi} H^{l}(s, X(s), V(s)) \mathrm{d} s .
$$

Note that as $t \uparrow t_{\mathbf{b}},(X(0 ; t, x, v), V(0 ; t, x, v)) \rightarrow\left(x_{\mathbf{b}}, v_{\mathbf{b}}\right) \in \gamma_{-}$. Therefore

$$
\lim _{t \uparrow t_{\mathbf{b}}} f_{-}^{l}(t, x, v)=e^{-\int_{0}^{t} \psi} f_{0}^{l}\left(x_{\mathbf{b}}, v_{\mathbf{b}}\right)+\int_{0}^{t} e^{-\int_{s}^{t} \psi} H^{l}(s, X(s), V(s)) \mathrm{d} s .
$$

Finally (2.66), (2.74), and above two limits imply that, as $l \rightarrow \infty$,

$$
\begin{aligned}
& \left\|f_{+}^{l}(t, x, v)-f_{-}^{l}(t, x, v)\right\|_{L^{1}} \\
= & \left\|e^{-\int_{t-t_{\mathbf{b}}}^{t} \psi} g^{l}\left(t-t_{\mathbf{b}}, x_{\mathbf{b}}, v_{\mathbf{b}}\right)-e^{-\int_{0}^{t} \psi} f_{0}^{l}(X(0), V(0))\right\|_{L^{1}}+O\left(t-t_{\mathbf{b}}\right) \\
\lesssim & \left\|e^{-\int_{0}^{t} \psi}\left[g\left(t-t_{\mathbf{b}}, x_{\mathbf{b}}, v_{\mathbf{b}}\right)-f_{0}\left(x_{\mathbf{b}}, v_{\mathbf{b}}\right)\right]\right\|_{L^{1}}+O\left(t-t_{\mathbf{b}}\right) \\
\rightarrow & 0 .
\end{aligned}
$$

Therefore the first term of RHS in (2.77) converges to zero as $l \rightarrow \infty$. From (2.76) the second term of RHS in (2.77) converges to the RHS of (2.71). This proves (2.71).

\section{Desingularization via Mixing in the Velocity space}

The main purpose of this section is proving Proposition 3

Proposition 3. Assume $E(t, x) \in C_{x}^{1}$ is given, and both 2.7) and (2.50) hold, and

$$
\sup _{t \geq 0} e^{\Lambda_{2} t}\left\|\nabla_{x} E(t)\right\|_{\infty} \leq \delta_{2} \ll 1 \text {. }
$$

Then for all $0<\sigma<1$ and $N>1$ and for all $s \geq 0, x \in \bar{\Omega}$,

$$
\int_{|u| \leq N} \frac{\mathrm{d} u}{\alpha_{f, \varepsilon}(s, x, u)^{\sigma}} \lesssim \sigma, \Omega, \Lambda_{1}, \delta_{1}, \Lambda_{2}, \delta_{2}, N 1
$$

and, for any $0<\kappa \leq 2$,

$$
\int_{|u| \geq N} \frac{e^{-C|v-u|^{2}}}{|v-u|^{2-\kappa}} \frac{1}{\alpha_{f, \varepsilon}(s, x, u)^{\sigma}} \mathrm{d} u \lesssim_{\sigma, \Omega, \Lambda_{1}, \delta_{1}, \Lambda_{2}, \delta_{2}, N, \kappa} 1 .
$$

The key element of the proof is the next change of variable formula.

Lemma 9. Assume (2.50) and (3.1) and $\delta_{2}\left(\Lambda_{2}\right)^{-2} \ll 1$. For $x \in \bar{\Omega}$ and $t \geq 0$, we define a map

$$
u \in\left\{u \in \mathbb{R}^{3}: t_{\mathbf{b}}(t, x, u) \leq t+1\right\} \mapsto\left(t_{\mathbf{b}}(t, x, u), x_{\mathbf{b}}(t, x, u)\right) \in \mathbb{R} \times \partial \Omega,
$$

with $\operatorname{det}\left(\frac{\partial\left(t_{\mathbf{b}}(t, x, u), x_{\mathbf{b}}(t, x, u)\right)}{\partial u}\right) \gtrsim \frac{t_{\mathbf{b}}(t, x, u)^{3}}{\left|n\left(x_{\mathbf{b}}(t, x, u)\right) \cdot v_{\mathbf{b}}(t, x, u)\right|}$.

For $g \geq 0$ and all $x \in \bar{\Omega}$ and $t \geq 0$

$$
\begin{aligned}
& \int_{\mathbb{R}^{3}} \mathbf{1}_{t_{\mathbf{b}}(t, x, u)<t+1} g(t, x, u) \mathrm{d} u \\
\lesssim & \int_{\partial \Omega} \int \frac{\left|n\left(x_{\mathbf{b}}\right) \cdot v_{\mathbf{b}}(t, x, u)\right|}{\left|t_{\mathbf{b}}\right|^{3}} g\left(t, x, u\left(x_{\mathbf{b}}, t_{\mathbf{b}}\right)\right) \mathrm{d} t_{\mathbf{b}} \mathrm{d} S_{x_{\mathbf{b}}},
\end{aligned}
$$

where the lower end of $t_{\mathbf{b}}$-integration has a lower bound as

$$
t_{\mathbf{b}}(t, x, u) \geq \frac{\left|x_{\mathbf{b}}(t, x, u)-x\right|}{\max _{t-t_{\mathbf{b}}(t, x, u) \leq s \leq t}|V(s ; t, x, u)|} .
$$

We need the following lemma to prove Lemma 9 .

Lemma 10. Assume (3.1) with $\Lambda_{2}+\delta_{2}+\varepsilon \leq 1$. Then there exists $C>0$ such that

$$
\begin{aligned}
\left|\nabla_{v} X(s ; t, x, v)\right| \leq & C e^{C \delta_{2}\left(\Lambda_{2}\right)^{-2}}|t-s|, \\
& \quad \text { for all } \max \left(t-t_{\mathbf{b}}(t, x, v),-\varepsilon\right) \leq s \leq t .
\end{aligned}
$$


Proof. Recall that $\nabla_{v} X(t ; t, x, v)=\nabla_{v} x=0$ and $\nabla_{v} V(t ; t, x, v)=\nabla_{v} v=\operatorname{Id}_{3,3}$. From (2.69) we have

$$
\begin{aligned}
\frac{d}{d s}\left|\nabla_{v} X(s ; t, x, v)\right| & \lesssim\left|\nabla_{v} V(s ; t, x, v)\right| \\
\frac{d}{d s}\left|\nabla_{v} V(s ; t, x, v)\right| & \lesssim\left|\nabla_{x} E(s, X(s ; t, x, v))\right|\left|\nabla_{v} X(s ; t, x, v)\right| \\
& \lesssim \delta_{2} e^{-\Lambda_{2}|s|}\left|\nabla_{v} X(s ; t, x, v)\right| .
\end{aligned}
$$

Then

$$
\begin{aligned}
\left|\nabla_{v} X(s ; t, x, v)\right| & \lesssim \int_{s}^{t}\left|\nabla_{v} V\left(s^{\prime} ; t, x, v\right)\right| \mathrm{d} s^{\prime} \\
& \lesssim|t-s|+\int_{s}^{t} \int_{s^{\prime}}^{t} \delta_{2} e^{-\Lambda_{2}\left|s^{\prime \prime}\right|}\left|\nabla_{v} X\left(s^{\prime \prime} ; t, x, v\right)\right| \mathrm{d} s^{\prime \prime} \mathrm{d} s^{\prime} \\
& \lesssim|t-s|+\int_{s}^{t} \int_{s}^{s^{\prime \prime}} \delta_{2} e^{-\Lambda_{2}\left|s^{\prime \prime}\right|}\left|\nabla_{v} X\left(s^{\prime \prime} ; t, x, v\right)\right| \mathrm{d} s^{\prime} \mathrm{d} s^{\prime \prime} \\
& \lesssim|t-s|+\int_{s}^{t}\left|s^{\prime \prime}-s\right| \delta_{2} e^{-\Lambda_{2}\left|s^{\prime \prime}\right|}\left|\nabla_{v} X\left(s^{\prime \prime} ; t, x, v\right)\right| \mathrm{d} s^{\prime \prime}
\end{aligned}
$$

By the Gronwall's inequality (set $\tilde{s}:=t-s$ if necessary), there exists some $C \gg 1$ such that

$$
\begin{aligned}
\left|\nabla_{v} X(s ; t, x, v)\right| \lesssim|t-s| \exp ( & \int_{0}^{t}\left|s^{\prime \prime}-s\right| \delta_{2} e^{-\Lambda_{2}\left|s^{\prime \prime}\right|} \mathrm{d} s^{\prime \prime} \\
& \left.\quad+\int_{\min (s, 0)}^{0}\left|s^{\prime \prime}-s\right| \delta_{2} e^{-\Lambda_{2}\left|s^{\prime \prime}\right|} \mathrm{d} s^{\prime \prime}\right) \\
& \lesssim|t-s| \exp \left(\frac{\delta_{2}}{\left(\Lambda_{2}\right)^{2}} \int_{0}^{\infty}\left(\Lambda_{2} s^{\prime \prime}\right) e^{-\left(\Lambda_{2} s^{\prime \prime}\right)} \mathrm{d}\left(\Lambda_{2} s^{\prime \prime}\right)+(\varepsilon)^{2} \delta_{2}\right) \\
& \lesssim|t-s| e^{(\varepsilon)^{2} \delta_{2}} e^{C \delta_{2}\left(\Lambda_{2}\right)^{-2}} \\
& \lesssim|t-s| e^{C \delta_{2}\left(\Lambda_{2}\right)^{-2}}, \quad \text { for all } \max \left(t-t_{\mathbf{b}}(t, x, v),-\varepsilon\right) \leq s \leq t .
\end{aligned}
$$

By choosing $C \gg 1$ large enough we conclude (3.7).

Now we are ready to prove Lemma 9 .

Proof of Lemma 9, The lower bound (3.6) is a direct consequence of the identity

$$
x_{\mathbf{b}}(t, x, u)=x+\int_{t}^{t-t_{\mathbf{b}}(t, x, u)} V(s ; t, x, u) \mathrm{d} s .
$$

Now we prove the inequality of 3.5. Except measure zero set of $u$ (when $x \in \partial \Omega$ we only need to exclude $u \cdot n(x)=0)$ we have $n\left(x_{\mathbf{b}}(t, x, u)\right) \cdot v_{\mathbf{b}}(t, x, u) \neq 0$ from Lemma 2. Then by the implicit function theorem $t_{\mathbf{b}}$ and $x_{\mathbf{b}}$ are differentiable locally.

Let us choose $p \in \partial \Omega$ which is close to $x_{\mathbf{b}}(t, x, u)$. Recall (1.29) and the notations $v_{\|, i}$ and $v_{n}$ in (2.10) and (2.11). Here, we temporarily define three-dimensional local parametrization $\eta_{p}$ near $x_{\mathbf{b}}(t, x, u)$ so that it is consistent with boundary parametrization (1.29). We use $x_{\|, i}$ and $x_{n}$ for (2.9). Let us pick $p \in \partial \Omega$ very close to $x_{\mathbf{b}}(t, x, u) \in \partial \Omega$ and we define

$$
\begin{aligned}
\eta_{p}:\left\{\left(x_{\|, 1}, x_{\|, 2}, x_{n}\right)\right. & \left.\in \mathbb{R}^{3}: x_{n}>0\right\} \cap B\left(0 ; \delta_{1}^{\prime}\right) \rightarrow \Omega \cap B\left(p ; \delta_{2}^{\prime}\right), \\
& \left(x_{\|, 1}, x_{\|, 2}, x_{n}\right) \mapsto \eta_{p}\left(x_{\|, 1}, x_{\|, 2}, x_{n}\right), \\
\eta_{p}\left(x_{\|, 1}, x_{\|, 2}, x_{n}\right)= & \eta_{p}\left(x_{\|, 1}, x_{\|, 2}, 0\right)+x_{n}\left(-n\left(x_{\|, 1}, x_{\|, 2}\right)\right),
\end{aligned}
$$

for sufficiently small $\delta_{1}^{\prime}, \delta_{2}^{\prime} \ll 1$ and we assume $\left|p-x_{\mathbf{b}}(t, x, u)\right|<\delta_{2}^{\prime}$. We used notation $n\left(x_{\|, 1}, x_{\|, 2}\right)$ to denote $n\left(\eta_{p}\left(x_{\|, 1}, x_{\|, 2}, 0\right)\right)$. From the definition of (3.9), $\partial_{i} \eta_{p}:=\frac{\partial}{\partial x_{\|, i}} \eta_{p}$ belongs to tangential plane of the level set for fixed $x_{n} \geq 0$ and hence

$$
\left(\partial_{1} \eta_{p} \times \partial_{2} \eta_{p}\right) \| n\left(x_{\|, 1}, x_{\|, 2}\right) .
$$

To specify local parametrization of $x_{\mathbf{b}}(t, x, u)$, let us denote

$$
x_{\mathbf{b}}(t, x, u)=\eta_{p 2}\left(x_{\mathbf{b}, 1}, x_{\mathbf{b}, 2}, 0\right) .
$$


We compute that, with the standard notation $g_{p, i j}:=\partial_{i} \eta_{p} \cdot \partial_{j} \eta_{p}$,

$$
\begin{aligned}
& \operatorname{det}\left(\frac{\partial\left(x_{\mathbf{b}, 1}, x_{\mathbf{b}, 2}, t_{\mathbf{b}}\right)}{\partial u}\right) \\
& =\operatorname{det}\left[t_{\mathbf{b}} \operatorname{Id}_{3 \times 3}-\int_{t}^{t-t_{\mathbf{b}}} \int_{t}^{s} \nabla_{u}(E(\tau, X(\tau ; t, x, u))) \mathrm{d} \tau \mathrm{d} s\right] \\
& \times \operatorname{det}\left[\begin{array}{c}
\frac{-1}{\sqrt{g_{p, 11}\left(x_{\mathbf{b}}\right)}}\left[\frac{\left.\frac{\partial_{1} \eta_{p}\left(x_{\mathbf{b}}\right)}{\sqrt{g_{p, 11}\left(x_{\mathbf{b}}\right)}}+\frac{v_{\|, 1}\left(x_{\mathbf{b}}\right)}{v_{n}\left(x_{\mathbf{b}}\right)} \frac{\partial_{3} \eta_{p}\left(x_{\mathbf{b}}\right)}{\sqrt{g_{p}, 33}\left(x_{\mathbf{b}}\right)}\right]}{\sqrt{g_{p, 22}\left(x_{\mathbf{b}}\right)}}\left[\frac{\partial_{2} \eta_{p}\left(x_{\mathbf{b}}\right)}{\sqrt{g_{p, 22}\left(x_{\mathbf{b}}\right)}}+\frac{v_{\|, 2}\left(x_{\mathbf{b}}\right)}{v_{n}\left(x_{\mathbf{b}}\right)} \frac{\partial_{3} \eta_{p}\left(x_{\mathbf{b}}\right)}{\sqrt{g_{p, 33}\left(x_{\mathbf{b}}\right)}}\right]\right. \\
\frac{1}{v_{n}\left(x_{\mathbf{b}}\right)} \frac{\partial_{3} \eta_{p}\left(x_{\mathbf{b}}\right)}{\sqrt{g_{p, 33}\left(x_{\mathbf{b}}\right)}}
\end{array}\right],
\end{aligned}
$$

where we have used direct computations (See [15, 27, for details)

$$
\begin{gathered}
\frac{\partial x_{\mathbf{b}, i}}{\partial u_{j}}=-\left[t_{\mathbf{b}} e_{j}-\int_{t}^{t-t_{\mathbf{b}}} \int_{t}^{s} \partial_{u_{j}}(E(\tau, X(\tau ; t, x, u))) \mathrm{d} \tau \mathrm{d} s\right] \\
\frac{1}{\sqrt{g_{p, i i}\left(x_{\mathbf{b}}\right)}}\left[\frac{\partial_{i} \eta_{p}\left(x_{\mathbf{b}}\right)}{\sqrt{g_{p, i i}\left(x_{\mathbf{b}}\right)}}+\frac{v_{\|, i}\left(x_{\mathbf{b}}\right)}{v_{n}\left(x_{\mathbf{b}}\right)} \frac{\partial_{3} \eta_{p}\left(x_{\mathbf{b}}\right)}{\sqrt{g_{p, 33}\left(x_{\mathbf{b}}\right)}}\right] \\
\nabla_{u} t_{\mathbf{b}}=\frac{1}{v_{n}\left(x_{\mathbf{b}}\right)}\left[t_{\mathbf{b}} I_{3 \times 3}-\int_{t}^{t-t_{\mathbf{b}}} \int_{t}^{s} \nabla_{u}(E(\tau, X(\tau ; t, x, u))) \mathrm{d} \tau \mathrm{d} s\right] \\
\cdot \frac{\partial_{3} \eta_{p}}{\sqrt{g_{p, 33}}} .
\end{gathered}
$$

Note that we have (3.7) from (3.1) and Lemma 10, Then, from (3.7) and (3.1),

$$
\begin{aligned}
& \left|\int_{t}^{t-t_{\mathbf{b}}} \int_{t}^{s} \nabla_{u}(E(\tau, X(\tau ; t, x, u))) \mathrm{d} \tau \mathrm{d} s\right| \\
\leq & \int_{t-t_{\mathbf{b}}}^{t} \int_{s}^{t}\left|\nabla_{u} X(\tau ; t, x, u)\right|\left|\nabla_{x} E(\tau, X(\tau ; t, x, u))\right| \mathrm{d} \tau \mathrm{d} s \\
\leq & C e^{C \delta_{2}\left(\Lambda_{2}\right)^{-2}} \delta_{2} \int_{t-t_{\mathbf{b}}}^{t} \int_{s}^{t}|t-\tau| e^{-\Lambda_{2} \tau} \mathrm{d} \tau \mathrm{d} s \\
\leq & C e^{C \delta_{2}\left(\Lambda_{2}\right)^{-2}} \delta_{2} t_{\mathbf{b}} \int_{t-t_{\mathbf{b}}}^{t} \int_{s}^{t} e^{-\Lambda_{2} \tau} \mathrm{d} \tau \mathrm{d} s \\
\leq & C e^{C \delta_{2}\left(\Lambda_{2}\right)^{-2}} \delta_{2} t_{\mathbf{b}} \times \frac{1}{\left(\Lambda_{2}\right)^{2}},
\end{aligned}
$$

where we have used a direct computation

$$
\begin{aligned}
\int_{t-t_{\mathbf{b}}}^{t} \int_{s}^{t} e^{-\Lambda_{2} \tau} \mathrm{d} \tau \mathrm{d} s & =\int_{t-t_{\mathbf{b}}}^{t} \frac{e^{-\Lambda_{2} t}-e^{-\Lambda_{2} s}}{-\Lambda_{2}} \mathrm{~d} s \\
& =\frac{e^{-\Lambda_{2} t}}{-\Lambda_{2}} t_{\mathbf{b}}-\frac{e^{-\Lambda_{2} t}-e^{-\Lambda_{2}\left(t-t_{\mathbf{b}}\right)}}{\left(\Lambda_{2}\right)^{2}} \\
& =\frac{e^{-\Lambda_{2}\left(t-t_{\mathbf{b}}\right)}}{\left(-\Lambda_{2}\right)^{2}}\left\{1-e^{-\Lambda_{2} t_{\mathbf{b}}}\left(1+\Lambda_{2} t_{\mathbf{b}}\right)\right\} \\
& \leq \frac{1}{\left(\Lambda_{2}\right)^{2}} .
\end{aligned}
$$
by

Therefore we derive a lower bound of the first determinant of (3.10) for $\delta_{2}\left(\Lambda_{2}\right)^{-2} \ll 1$, which is guaranteed

$$
\left(t_{\mathbf{b}}\right)^{3} \operatorname{det}\left[I_{3 \times 3}+\frac{C \delta_{2}}{\left(\Lambda_{2}\right)^{2}} e^{C \delta_{2}\left(\Lambda_{2}\right)^{-2}}\right] \geq \frac{\left(t_{\mathbf{b}}\right)^{3}}{2},
$$

where we have used $\operatorname{det}\left[I_{n \times n}+O(\delta)\right]=1+O_{n}(\delta)$ for $0<\delta \ll 1$. 
Clearly the second determinant of (3.10) equals

$$
\begin{aligned}
& \mid \frac{1}{v_{n}\left(x_{\mathbf{b}}\right)} \frac{\partial_{3} \eta_{p}\left(x_{\mathbf{b}}\right)}{\sqrt{g_{p, 33}\left(x_{\mathbf{b}}\right)}} \\
& \cdot\left(\frac{-1}{\sqrt{g_{p, 11}\left(x_{\mathbf{b}}\right)}}\left[\frac{\partial_{1} \eta_{p}\left(x_{\mathbf{b}}\right)}{\sqrt{g_{p, 11}\left(x_{\mathbf{b}}\right)}}+\frac{v_{\|, 1}\left(x_{\mathbf{b}}\right)}{v_{n}\left(x_{\mathbf{b}}\right)} \frac{\partial_{3} \eta_{p}\left(x_{\mathbf{b}}\right)}{\sqrt{g_{p, 33}\left(x_{\mathbf{b}}\right)}}\right]\right. \\
& \left.\quad \times \frac{-1}{\sqrt{g_{p, 22}\left(x_{\mathbf{b}}\right)}}\left[\frac{\partial_{2} \eta_{p}\left(x_{\mathbf{b}}\right)}{\sqrt{g_{p, 22}\left(x_{\mathbf{b}}\right)}}+\frac{v_{\|, 2}\left(x_{\mathbf{b}}\right)}{v_{n}\left(x_{\mathbf{b}}\right)} \frac{\partial_{3} \eta_{p}\left(x_{\mathbf{b}}\right)}{\sqrt{g_{p, 33}\left(x_{\mathbf{b}}\right)}}\right]\right) \mid \\
& =\frac{1}{\sqrt{g_{p, 11}\left(x_{\mathbf{b}}\right)} \sqrt{g_{p, 22}\left(x_{\mathbf{b}}\right)}} \times \frac{1}{\left|v_{n}\left(x_{\mathbf{b}}\right)\right|} .
\end{aligned}
$$

Now we check whether the mapping $u \rightarrow\left(x_{\mathbf{b}}(t, x, u), t_{\mathbf{b}}(t, x, u)\right)$ is one-to-one for all $t \in \mathbb{R}, t_{\mathbf{b}}(t, x, u)<N$, and $n\left(x_{\mathbf{b}}(t, x, u)\right) \cdot v_{\mathbf{b}}(t, x, u)<-\frac{1}{N}$ with $x \in \Omega$ or $x \in \partial \Omega$ with $n(x) \cdot u>0$. Assume that there exist $u$ and $\tilde{u}$ with such conditions and satisfy $x_{\mathbf{b}}(t, x, u)=x_{\mathbf{b}}(t, x, \tilde{u})$ and $t_{\mathbf{b}}(t, x, u)=t_{\mathbf{b}}(t, x, \tilde{u})$. As (3.10) we choose $p \in \partial \Omega$ near $x_{\mathbf{b}}(t, x, u)$ and use the same parametrization. Then by an expansion, for some $\bar{u} \in \overline{\tilde{u} u}$,

$$
0=\left[\begin{array}{c}
x_{\mathbf{b}, 1}(t, x, \tilde{u}) \\
x_{\mathbf{b}, 2}(t, x, \tilde{u}) \\
t_{\mathbf{b}}(t, x, \tilde{u})
\end{array}\right]-\left[\begin{array}{c}
x_{\mathbf{b}, 1}(t, x, u) \\
x_{\mathbf{b}, 2}(t, x, u) \\
t_{\mathbf{b}}(t, x, u)
\end{array}\right]=\left[\begin{array}{c}
\nabla_{u} x_{\mathbf{b}, 1}(t, x, \bar{u}) \\
\nabla_{u} x_{\mathbf{b}, 2}(t, x, \bar{u}) \\
\nabla_{u} t_{\mathbf{b}}(t, x, \bar{u})
\end{array}\right](\tilde{u}-u)
$$

This equality can be true only if the determinant of the Jacobian matrix equals zero. And (3.13) and (3.14) imply that $t_{\mathbf{b}}(t, x, \bar{u})=0$. But this implies $x \in \partial \Omega$ and hence $v_{\mathbf{b}}(t, x, u)=u$ and $v_{\mathbf{b}}(t, x, \tilde{u})=\tilde{u}$. Then $n(x) \cdot u<0$ and $n(x) \cdot \tilde{u}<0$ which are out of our domain.

Now we apply the change of variables to conclude the proof of Lemma 9.

Finally we present the proof of the main result of this section.

Proof of Proposition [3. Step 1. We apply Lemma 9 and deduce that

$$
\int_{|u| \leq N} \frac{\mathbf{1}_{t_{\mathbf{b}}(s, x, u)<t+1}}{\alpha_{f, \varepsilon}(s, x, u)^{\sigma}} \mathrm{d} u \leq \int_{\partial \Omega} \int \frac{\left|n\left(x_{\mathbf{b}}(t, x, u)\right) \cdot v_{\mathbf{b}}(t, x, u)\right|^{1-\sigma}}{\left|t_{\mathbf{b}}(t, x, u)\right|^{3}} \mathrm{~d} t_{\mathbf{b}} \mathrm{d} x_{\mathbf{b}} .
$$

Here $t_{\mathbf{b}}$ has a lower bound from (3.6)

$$
t_{\mathbf{b}} \geq \frac{\left|x_{\mathbf{b}}-x\right|}{N+\delta_{1} / \Lambda_{1}}
$$

where we have used the fact, from $E$ in (2.50), for $|u| \leq N$ and $0 \leq s \leq t$,

$$
\begin{aligned}
|V(s ; t, x, u)| & \leq|u|+\int_{0}^{t}|E(s, X(s ; t, x, u))| \mathrm{d} s \\
& \leq N+\int_{0}^{t} \delta_{1} e^{-\Lambda_{1} s} \mathrm{~d} s \\
& \leq N+\delta_{1} / \Lambda_{1} .
\end{aligned}
$$

From $V(s ; t, x, u)=v_{\mathbf{b}}(t, x, u)+\int_{t-t_{\mathbf{b}}(t, x, u)}^{s} E(\tau, X(\tau ; t, x, u)) \mathrm{d} \tau$, we have

$$
\begin{aligned}
& x_{\mathbf{b}}(t, x, u)-x \\
= & \int_{t}^{t-t_{\mathbf{b}}(t, x, u)}\left\{v_{\mathbf{b}}(t, x, u)+\int_{t-t_{\mathbf{b}}(t, x, u)}^{s} E(\tau, X(\tau ; t, x, u)) \mathrm{d} \tau\right\} \mathrm{d} s \\
= & -t_{\mathbf{b}}(t, x, u) v_{\mathbf{b}}(t, x, u) \\
& +\int_{t}^{t-t_{\mathbf{b}}(t, x, u)} \int_{t-t_{\mathbf{b}}(t, x, u)}^{s} E(\tau, X(\tau ; t, x, u)) \mathrm{d} \tau \mathrm{d} s .
\end{aligned}
$$

Therefore we can conclude that

$$
\begin{aligned}
& v_{\mathbf{b}}(t, x, u) \\
= & \frac{x-x_{\mathbf{b}}(t, x, u)}{t_{\mathbf{b}}(t, x, u)} \\
& -\frac{1}{t_{\mathbf{b}}(t, x, u)} \int_{t-t_{\mathbf{b}}(t, x, u)}^{t} \int_{t-t_{\mathbf{b}}(t, x, u)}^{s} E(\tau, X(\tau ; t, x, u)) \mathrm{d} \tau \mathrm{d} s,
\end{aligned}
$$


and hence

$$
\begin{aligned}
& \left|n\left(x_{\mathbf{b}}(t, x, u)\right) \cdot v_{\mathbf{b}}(t, x, u)\right| \\
\leq & \frac{\left|\left(x-x_{\mathbf{b}}(t, x, u)\right) \cdot n\left(x_{\mathbf{b}}(t, x, u)\right)\right|}{t_{\mathbf{b}}(t, x, u)} \\
& +\frac{t_{\mathbf{b}}(t, x, u)}{2} \max _{t-t_{\mathbf{b}}(t, x, u) \leq \tau \leq t}\left|E(\tau, X(\tau ; t, x, u)) \cdot n\left(x_{\mathbf{b}}(t, x, u)\right)\right| .
\end{aligned}
$$

Using (3.18), we further bound (3.16) by

$$
\begin{aligned}
(3.16) \leq & \int_{\partial \Omega}\left|\left(x-x_{\mathbf{b}}(t, x, u)\right) \cdot n\left(x_{\mathbf{b}}(t, x, u)\right)\right|^{1-\sigma} \int \frac{1}{t_{\mathbf{b}}^{4-\sigma}} \mathrm{d} t_{\mathbf{b}} \mathrm{d} S_{x_{\mathbf{b}}} \\
& +\|E\|_{L^{\infty}} \int_{\partial \Omega} \int \frac{1}{t_{\mathbf{b}}^{2+\sigma}} \mathrm{d} t_{\mathbf{b}} \mathrm{d} S_{x_{\mathbf{b}}},
\end{aligned}
$$

where $t_{\mathbf{b}}$ has a lower bound of (3.17).

By integrating over $t_{\mathrm{b}}$ in (3.19) and using the lower bound (3.17), we deduce that

$$
(13.19) \lesssim \int_{\partial \Omega}\left|\left(x-x_{\mathbf{b}}\right) \cdot n\left(x_{\mathbf{b}}\right)\right|^{1-\sigma} \frac{\left(N+\delta_{1} / \Lambda_{1}\right)^{3-\sigma}}{\left|x-x_{\mathbf{b}}\right|^{3-\sigma}} \mathrm{d} S_{x_{\mathbf{b}}} .
$$

Recall (1.29) and choose $p \in \partial \Omega$ such that $x_{\mathbf{b}}$ is close to $p$ locally. Then locally (3.21) is bounded by

$$
\begin{aligned}
& \left(N+\delta_{1} / \Lambda_{1}\right)^{3-\sigma} \\
& \times \int_{\left|\left(y_{\|, 1}, y_{\|, 2}\right)\right| \ll 1} \frac{\left|\left(x-\eta_{p}\left(y_{\|, 1}, y_{\|, 2}, 0\right)\right) \cdot n\left(y_{\|, 1}, y_{\|, 2}\right)\right|^{1-\sigma}}{\left|x-\eta_{p}\left(y_{\|, 1}, y_{\|, 2}, 0\right)\right|^{3-\sigma}} \mathrm{d} y_{\|, 1} \mathrm{~d} y_{\|, 2} .
\end{aligned}
$$

Without loss of generality we may assume $\left|x-\eta_{p}\left(y_{\|, 1}, y_{\|, 2}, 0\right)\right| \ll 1$, otherwise the denominator of (3.22) has a lower bound. Hence $x$ is close to $p$ and we can parametrize $x=\eta_{p}\left(x_{\|, 1}, x_{\|, 2}, 0\right)-x_{n} n_{p}\left(x_{\|, 1}, x_{\|, 2}\right)$ as (2.9) or (3.9). By a re-parametrization we may assume that the coordinate of $p \in \partial \Omega$ is $\left(y_{\|}, y_{n}\right)=\left(y_{\|, 1}, y_{\|, 2}, y_{n}\right)=(0,0,0)$. Therefore we can assume $\left(x_{\|, 1}, x_{\|, 2}, x_{n}\right) \sim(0,0,0) \sim\left(y_{\|, 1}, y_{\|, 2}, 0\right)$.

We expand

$$
\begin{aligned}
& \left(x-\eta_{p}\left(y_{\|}, 0\right)\right) \cdot\left(-n_{p}\left(y_{\|}\right)\right) \\
= & \left(x_{n}\left(-n_{p}\left(x_{\|}\right)\right)+\left(\eta_{p}\left(x_{\|}, 0\right)-\eta_{p}\left(y_{\|}, 0\right)\right)\right) \cdot\left(-n_{p}\left(y_{\|}\right)\right) \\
= & \left(\left(-n_{p}\left(y_{\|}\right)\right)-\int_{0}^{1}\left(x_{\|}-y_{\|}\right) \cdot \nabla_{x_{\|}} n_{p}\left(s x_{\|}+(1-s) y_{\|}\right) \mathrm{d} s\right) \cdot\left(-n_{p}\left(y_{\|}\right)\right) \times x_{n} \\
& +\left(\int_{0}^{1}\left(x_{\|}-y_{\|}\right) \cdot \nabla_{x_{\|}} \eta_{p}\left(s x_{\|}+(1-s) y_{\|}, 0\right) \mathrm{d} s\right) \cdot\left(-n_{p}\left(y_{\|}\right)\right) \\
= & x_{n}+O_{\left\|n_{p}\right\|_{C^{1}}}\left(\left|x_{\|}-y_{\|} \| x_{n}\right|\right) \\
& +\left(x_{\|}-y_{\|}\right) \cdot \nabla_{x_{\|}} \eta_{p}\left(y_{\|}, 0\right) \cdot\left(-n_{p}\left(y_{\|}\right)\right) \\
& -n_{p}\left(y_{\|}\right) \cdot\left(\int_{0}^{1} \int_{0}^{s}\left(x_{\|}-y_{\|}\right) \cdot \nabla_{x_{\|}}^{2} \eta_{p}\left(\tau x_{\|}+(1-\tau) y_{\|}, 0\right) \cdot\left(x_{\|}-y_{\|}\right) \mathrm{d} \tau \mathrm{d} s\right) \\
= & x_{n}+O_{\left\|\eta_{p}\right\|_{C^{2}}}\left(\left|x_{\|}-y_{\|}\right|\right)\left|x_{n}\right|+O_{\left\|\eta_{p}\right\|_{C^{2}}}\left(\left|x_{\|}-y_{\|}\right|^{2}\right),
\end{aligned}
$$

where we have used the fact, at the cancellation above,

$$
\nabla_{x_{\|}} \eta_{p}\left(y_{\|}, 0\right) \perp n_{p}\left(y_{\|}\right) .
$$

Clearly, for $\left|x_{\|}-y_{\|}\right| \ll 1$,

$$
\begin{aligned}
& \frac{1}{2}\left(\left|x_{n}\right|-C_{\left\|\eta_{p}\right\|_{C^{2}}}\left|x_{\|}-y_{\|}\right|^{2}\right) \\
\leq & \left|\left(x-\eta_{p}\left(y_{\|}, 0\right)\right) \cdot\left(-n_{p}\left(y_{\|}\right)\right)\right| \leq 2\left(\left|x_{n}\right|+C_{\left\|\eta_{p}\right\|_{C^{2}}}\left|x_{\|}-y_{\|}\right|^{2}\right) .
\end{aligned}
$$


On the other hand, for $i=1,2$,

$$
\begin{aligned}
& \left(x-\eta_{p}\left(y_{\|}, 0\right)\right) \cdot \frac{\partial_{i} \eta_{p}\left(y_{\|}, 0\right)}{\sqrt{g_{p, i i}\left(y_{\|}, 0\right)}} \\
= & \left(x_{n}\left(-n_{p}\left(x_{\|}\right)\right)+\left(\eta_{p}\left(x_{\|}, 0\right)-\eta_{p}\left(y_{\|}, 0\right)\right)\right) \cdot \frac{\partial_{i} \eta_{p}\left(y_{\|}, 0\right)}{\sqrt{g_{p, i i}\left(y_{\|}, 0\right)}} \\
= & \left(\left(-n_{p}\left(y_{\|}\right)\right)-\int_{0}^{1}\left(x_{\|}-y_{\|}\right) \cdot \nabla_{x_{\|}} n_{p}\left(s x_{\|}+(1-s) y_{\|}\right) \mathrm{d} s\right) \cdot \frac{\partial_{i} \eta_{p}\left(y_{\|}, 0\right)}{\sqrt{g_{p, i i}\left(y_{\|}, 0\right)}} x_{n} \\
& +\left(\int_{0}^{1}\left(x_{\|}-y_{\|}\right) \cdot \nabla_{x_{\|}} \eta_{p}\left(s x_{\|}+(1-s) y_{\|}, 0\right) \mathrm{d} s\right) \cdot \frac{\partial_{i} \eta_{p}\left(y_{\|}, 0\right)}{\sqrt{g_{p, i i}\left(y_{\|}, 0\right)}} \\
= & \left.\left(-n_{p}\left(y_{\|}\right)\right) \cdot \frac{\partial_{i} \eta_{p}\left(y_{\|}, 0\right)}{\sqrt{g_{p, i i}\left(y_{\|}, 0\right)}} x_{n}+O_{\left\|\eta_{p}\right\|_{C^{2}}\left(\mid x_{\|}\right.}-y_{\|} \mid\right) x_{n}, \quad f r o m \underline{(3.23)} \\
& +\left(\left(x_{\|}-y_{\|}\right) \cdot \nabla_{x_{\|}} \eta_{p}\left(y_{\|}, 0\right)\right) \cdot \frac{\partial_{i} \eta_{p}\left(y_{\|}, 0\right)}{\sqrt{g_{p, i i}\left(y_{\|}, 0\right)}}+O_{\left\|p_{p}\right\|_{C^{2}}}\left(\left|x_{\|}-y_{\|}\right|^{2}\right) .
\end{aligned}
$$

Hence, for $\left|x_{n}\right|,\left|x_{\|}-y_{\|}\right| \ll 1$,

$$
\begin{aligned}
& \left(\sum_{i=1,2}\left|\left(x-\eta_{p}\left(y_{\|}, 0\right)\right) \cdot \frac{\partial_{i} \eta_{p}\left(y_{\|}, 0\right)}{\sqrt{g_{p, i i}\left(y_{\|}, 0\right)}}\right|^{2}\right)^{1 / 2} \\
= & \left|x_{\|}-y_{\|}\right|+O_{\left\|\eta_{p}\right\|_{C^{2}}}\left(\left|x_{\|}-y_{\|}\right|\right) x_{n}+O_{\left\|\eta_{p}\right\|_{C^{2}}}\left(\left|x_{\|}-y_{\|}\right|^{2}\right) \\
\geq & \frac{1}{2}\left|x_{\|}-y_{\|}\right| .
\end{aligned}
$$

From (3.24) and (3.25), we derive an upper bound of the integrand of (3.22) and then split it as

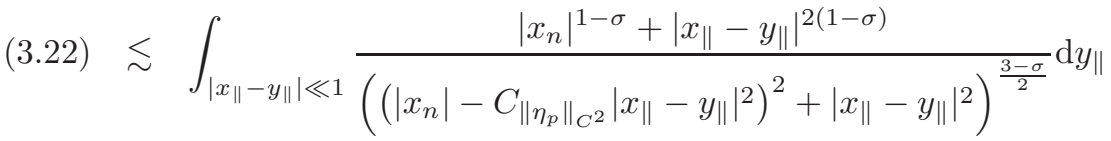

$$
\begin{aligned}
& =\int_{\left|x_{\|}-y_{\|}\right| \leq\left|x_{n}\right|}+\int_{\left|x_{n}\right| \leq\left|x_{\|}-y_{\|}\right|} .
\end{aligned}
$$

First we consider the case of $\left|x_{\|}-y_{\|}\right| \leq\left|x_{n}\right|$. For $\left|x_{\|}-y_{\|}\right| \ll 1$,

$$
\left|x_{n}\right|-C_{\left\|\eta_{p}\right\|_{C^{2}}}\left|x_{\|}-y_{\|}\right|^{2} \geq \frac{\left|x_{n}\right|}{2} .
$$

Hence, by a change of variables $R:=\left|x_{\|}-y_{\|}\right|^{2}$,

$$
\begin{aligned}
& \int_{\left|x_{\|}-y_{\|}\right| \leq\left|x_{n}\right|} \text { in (3.26) } \\
\lesssim & \int_{0}^{\left|x_{n}\right|^{2}} \frac{\left|x_{n}\right|^{1-\sigma}+R^{1-\sigma}}{\left[\left|x_{n}\right|^{2} / 4+R\right]^{\frac{3-\sigma}{2}}} \mathrm{~d} R \\
\lesssim & \int_{0}^{\left|x_{n}\right|^{2}} \frac{\left|x_{n}\right|^{1-\sigma}}{\left|x_{n}\right|^{3-\sigma}} \mathrm{d} R+\int_{0}^{\left|x_{n}\right|^{2}} R^{-\frac{1}{2}-\frac{\sigma}{2}} \mathrm{~d} R \\
\lesssim & 1+\left|x_{n}\right|^{1-\sigma} .
\end{aligned}
$$

On the other hand, from the same change of variables,

$$
\begin{aligned}
& \left.\int_{\left|x_{n}\right| \leq\left|x_{\|}-y_{\|}\right|} \text {in } \sqrt{3.26}\right) \\
\lesssim & \int_{\left|x_{n}\right|^{2}}^{1} \frac{\left|x_{n}\right|^{1-\sigma}}{R^{\frac{3-\sigma}{2}}} \mathrm{~d} R+\int_{\left|x_{n}\right|^{2}}^{1} R^{-\frac{1}{2}-\frac{\sigma}{2}} \mathrm{~d} R \\
\lesssim & 1+\left|x_{n}\right|^{1-\sigma} .
\end{aligned}
$$

From (3.27) to (3.28), and (3.21), (3.22), we conclude (3.19) $\lesssim 1$. 
In a similar way as deriving (3.21), using (3.17), we bound

$$
\text { (3.20) } \lesssim \frac{\|E\|_{L^{\infty}}\left(N+\delta_{1} / \Lambda_{1}\right)^{1+\sigma}}{1+\sigma} \int_{\partial \Omega} \frac{\mathrm{d} S_{x_{\mathbf{b}}}}{\left|x_{\mathbf{b}}-x\right|^{1+\sigma}}
$$

Since $x_{\mathbf{b}}$ varies in the 2 dimensional smooth hypersurface $\partial \Omega$, we can easily see that $\frac{1}{\left|x_{\mathbf{b}}-x\right|^{1+\sigma}}$ is integrable for $0<\sigma<1$ and hence (3.20) is bounded. This estimate together with (3.28) derives a bound of (3.16) and hence (3.2).

Step 2. Now we consider (3.3). From $|u| \geq N \gg 1$ and (2.50), we have

$$
\begin{aligned}
|X(s ; t, x, u)-x| & =\left|\int_{s}^{t} V\left(s^{\prime} ; t, x, u\right) \mathrm{d} s^{\prime}\right| \\
& \geq|u||t-s|-\int_{s}^{t} \int_{s^{\prime}}^{t} e^{-\Lambda_{1} s^{\prime \prime}} \delta_{1} \mathrm{~d} s^{\prime \prime} \mathrm{d} s^{\prime} \\
& \geq\left(|u|-\Lambda_{1}{ }^{-1} \delta_{1}\right)|t-s| .
\end{aligned}
$$

If $N>2 \Lambda_{1}^{-1} \delta_{1}$ then $|X(s ; t, x, u)-x| \geq \frac{|u|}{2}|t-s|$. Since the domain $\Omega$ is bounded, we have $\sup |X(s ; t, x, u)-x|<$ $\infty$. Finally we conclude that

$$
\begin{array}{lll}
|u| t_{\mathbf{b}}(t, x, u) \lesssim \Omega 1 & \text { for } & |u| \geq N \\
t_{\mathbf{b}}(t, x, u) \lesssim \Omega, N 1 & \text { for } & |u| \geq N .
\end{array}
$$

Furthermore from (3.29), for $|u| \geq N$

$$
\begin{aligned}
& \int_{s-t_{\mathbf{b}}(s, x, u)}^{s}\left|V\left(s^{\prime} ; s, x, u\right)\right| \mathrm{d} s^{\prime} \\
& \leq|u| t_{\mathbf{b}}(s, x, u)+\int_{s-t_{\mathbf{b}}(s, x, u)}^{s} \int_{s^{\prime}}^{s}\left|E\left(s^{\prime \prime}, X\left(s^{\prime \prime} ; s, x, u\right)\right)\right| \mathrm{d} s^{\prime \prime} \mathrm{d} s^{\prime} \\
& \int_{\Omega} 1+\int_{s-t_{\mathbf{b}}(s, x, u)}^{s} \int_{s^{\prime}}^{s} \delta_{1} e^{-\Lambda_{1} s^{\prime \prime}} \mathrm{d} s^{\prime \prime} \mathrm{d} s^{\prime}, \quad \text { from } \\
& \delta_{\Omega} 1+\frac{\delta_{1}}{\left(\Lambda_{1}\right)^{2}}, \quad \text { from } \\
& \lesssim_{\Omega, K, \Lambda_{1}, \delta_{1}} 1 .
\end{aligned}
$$

Step 3. Now we derive a version of velocity lemma (See several versions of velocity lemma in [19, 23, 17, [15]). Without loss of generality we assume that there exists $\xi: \mathbb{R}^{3} \rightarrow \mathbb{R}$ such that $\Omega=\left\{x \in \mathbb{R}^{3}: \xi(x)<0\right\}$ and $\nabla \xi(x) \neq 0$ when $\xi(x)=0$ (See the construction of such $\xi$ in (2.3) of [8]). We define

$$
\tilde{\alpha}(t, x, v):=\sqrt{\xi(x)^{2}+|\nabla \xi(x) \cdot u|^{2}-2\left(u \cdot \nabla_{x}^{2} \xi(x) \cdot u\right) \xi(x)} .
$$

For $|u| \geq N$ and $t-t_{\mathbf{b}}(t, x, u) \geq-\varepsilon / 2$,

$$
\alpha_{f, \varepsilon}(t, x, u)^{2} \lesssim_{\Omega, N, \delta_{1}, \Lambda_{1}} \tilde{\alpha}(t, x, u)^{2} \lesssim_{\Omega, N, \delta_{1}, \Lambda_{1}} \alpha_{f, \varepsilon}(t, x, u)^{2} .
$$

We remark that the equivalent relationship fails in general for small $u$ as $N \downarrow 0$.

The proof of (3.33) is now given here: Assume $|u| \geq N$. Then for $\left(\Lambda_{1}\right)^{-1} \delta_{1} \leq 1$ and $t-t_{\mathbf{b}}(t, x, u) \leq s \leq t$

$$
N / 2 \leq N-\Lambda_{1}^{-1} \delta_{1} \leq|V(s ; t, x, u)| \leq N+\Lambda_{1}^{-1} \delta_{1} \leq 2 N .
$$

From a direct computation,

$$
\begin{aligned}
& {\left[\partial_{t}+u \cdot \nabla_{x}+E(t, x) \cdot \nabla_{u}\right]\left\{\xi(x)^{2}+|\nabla \xi(x) \cdot u|^{2}-2\left(u \cdot \nabla_{x}^{2} \xi(x) \cdot u\right) \xi(x)\right\}} \\
& =2\{u \cdot \nabla \xi\} \xi+2\left\{u \cdot \nabla^{2} \xi-u\right\}\left\{u \cdot \nabla_{x} \xi\right\}-2 u \cdot\left(u \cdot \nabla \nabla^{2} \xi \cdot u\right) \xi \\
& \quad \frac{-2\left\{u \cdot \nabla^{2} \xi \cdot u\right\}\{u \cdot \nabla \xi\}}{2}+2\{E \cdot \nabla \xi\}\{\nabla \xi \cdot u\}-4\left\{E \cdot \nabla^{2} \xi \cdot u\right\} \xi \\
& \lesssim|u \cdot \nabla \xi|^{2}+|\xi|^{2}+\left\{|u|+\frac{1}{|u|}\right\}\left(-2\left(u \cdot \nabla_{x}^{2} \xi(x) \cdot u\right) \xi(x)\right) \\
& \quad+|E \cdot \nabla \xi||\nabla \xi \cdot u| .
\end{aligned}
$$


From the Neumann BC $(n(x) \cdot E(t, x)=0$ on $x \in \partial \Omega)$, we have

$$
\begin{aligned}
& |E(t, x) \cdot \nabla \xi(x)| \\
& \leq\left|E\left(t, x_{*}\right) \cdot \nabla \xi\left(x_{*}\right)\right|+\|E(t)\|_{C^{1}(\bar{\Omega})}\|\xi\|_{C^{2}(\bar{\Omega})}\left|x-x_{*}\right| \\
& \lesssim_{\Omega}\|E(t)\|_{C^{1}(\bar{\Omega})}|\xi(x)|,
\end{aligned}
$$

where $x_{*} \in \partial \Omega$ such that $\left|x-x_{*}\right|=\inf _{y \in \partial \Omega}|x-y|$.

By controlling the last term of (3.35) by (3.36) and using (3.34), we conclude that

$$
\begin{aligned}
& \frac{d}{d s} \tilde{\alpha}(s, X(s ; t, x, u), V(s ; t, x, u))^{2} \\
& \lesssim_{\Omega}\left(1+|V(s ; t, x, u)|+\frac{1}{|V(s ; t, x, u)|}\right) \tilde{\alpha}(s, X(s ; t, x, u), V(s ; t, x, u))^{2} \\
& \lesssim_{\Omega, R, N}(1+|V(s ; t, x, u)|) \tilde{\alpha}(s, X(s ; t, x, u), V(s ; t, x, u))^{2} .
\end{aligned}
$$

Then, using (3.29), (3.30), and (3.31), we derive

$$
\begin{aligned}
& \tilde{\alpha}\left(t-t_{\mathbf{b}}(t, x, u), X\left(t-t_{\mathbf{b}}, t, x, u\right), V\left(t-t_{\mathbf{b}}, t, x, u\right)\right)^{2} \\
& \lesssim_{\Omega, N, \delta_{1}, \Lambda_{1}} \tilde{\alpha}(t, x, u)^{2} \\
& \lesssim_{\Omega, N, \delta_{1}, \Lambda_{1}} \tilde{\alpha}\left(t-t_{\mathbf{b}}(t, x, u), X\left(t-t_{\mathbf{b}}, t, x, u\right), V\left(t-t_{\mathbf{b}}, t, x, u\right)\right)^{2} .
\end{aligned}
$$

Note that from $\left(X\left(t-t_{\mathbf{b}}, t, x, u\right), V\left(t-t_{\mathbf{b}}, t, x, u\right)\right) \in \gamma_{-}$, (1.25), (3.32), and the fact $\nabla \xi(x) \neq 0$ when $\xi(x)=0$ (i.e. $x \in \partial \Omega$ ), we have

$$
\begin{aligned}
& \tilde{\alpha}\left(t-t_{\mathbf{b}}(t, x, u), X\left(t-t_{\mathbf{b}}, t, x, u\right), V\left(t-t_{\mathbf{b}}, t, x, u\right)\right) \\
\equiv & \left|\nabla \xi\left(X\left(t-t_{\mathbf{b}}, t, x, u\right)\right) \cdot V\left(t-t_{\mathbf{b}}, t, x, u\right)\right| \\
\sim & \left|n\left(X\left(t-t_{\mathbf{b}}, t, x, u\right)\right) \cdot V\left(t-t_{\mathbf{b}}, t, x, u\right)\right| \\
\sim & \alpha_{f, \varepsilon}(t, x, u), \quad \text { for } t-t_{\mathbf{b}}(t, x, u) \geq-\varepsilon / 2 .
\end{aligned}
$$

Here the equivalent relation " $\sim$ " depends on $\Omega$.

Combining the above estimate with (3.37) we finish the proof of (3.33).

Step 4. From (3.33) and (1.25), we have, for $|u| \geq N$,

$$
\tilde{\alpha}(t, x, u) \gtrsim \mathbf{1}_{t-t_{\mathbf{b}}(t, x, u) \geq-\varepsilon / 2}|\nabla \xi(x) \cdot u|+\mathbf{1}_{t-t_{\mathbf{b}}(t, x, u) \leq-\varepsilon / 2} .
$$

Therefore we conclude that

$$
\begin{aligned}
\text { (3.3) } \lesssim & 1+\int_{0}^{\infty} \frac{e^{-C|(v-u) \cdot \nabla \xi|^{2}}}{|\nabla \xi(x) \cdot u|^{\sigma}} \mathrm{d}(\nabla \xi(x) \cdot u) \\
& \quad \times \iint_{\mathbb{R}^{2}} \frac{1}{\left|u_{\perp}-v_{\perp}\right|^{2-\kappa}} e^{-C\left|v_{\perp}-u_{\perp}\right|^{2}} \mathrm{~d} u_{\perp} \\
\lesssim & 1+\int_{0}^{\infty} \frac{e^{-C|(v-u) \cdot \nabla \xi|^{2}}}{|\nabla \xi(x) \cdot u|^{\sigma}} \mathrm{d}(\nabla \xi(x) \cdot u) \times \int_{0}^{\infty} \frac{e^{-C|y|^{2}}}{|y|^{1-\kappa}} \mathrm{d}|y| \\
\lesssim & 1,
\end{aligned}
$$

where $u_{\perp}=u-\left(u \cdot \frac{\nabla \xi(x)}{|\nabla \xi(x)|}\right) \frac{\nabla \xi(x)}{|\nabla \xi(x)|}$.

\section{Nonlinear-Normed Energy Estimates in Weighted $W^{1, p}$}

The main result of this section is the following a priori estimate.

Proposition 4. Let us choose $0<\tilde{\vartheta}<\vartheta \ll 1$ and

$$
\frac{p-2}{p}<\beta<\frac{2}{3}, \quad \text { for } 3<p<6 .
$$

Assume $f$ solves (1.15), (1.16), 1.17), and $f \in L^{\infty}\left((0, T) ; L^{p}\left(\Omega \times \mathbb{R}^{3}\right)\right) \cap L^{1}\left((0, T) ; L^{p}(\gamma)\right)$ and $w_{\tilde{\vartheta}} \alpha_{f, \varepsilon}^{\beta} \nabla_{x, v} f \in$ $L^{\infty}\left((0, T) ; L^{p}\left(\Omega \times \mathbb{R}^{3}\right)\right) \cap L^{1}\left((0, T) ; L^{p}(\gamma)\right)$ and

$$
\sup _{0 \leq t \leq \infty}\left\|w_{\vartheta} f(t)\right\|_{\infty} \ll 1
$$


and

$$
\sup _{0 \leq t \leq T} e^{\Lambda_{1} t}\left\|\nabla_{x} \phi_{f}(t)\right\|_{\infty}<\delta_{1}
$$

with

$$
0<\frac{\delta_{1}}{\Lambda_{1}} \ll \Omega 1 .
$$

Then there exists $C_{p}>0$ such that

$$
\begin{aligned}
& \left\|w_{\tilde{\vartheta}} f(t)\right\|_{p}^{p}+\left\|w_{\tilde{\vartheta}} \alpha_{f, \varepsilon}^{\beta} \partial f(t)\right\|_{p}^{p} \\
& \leq C_{p} e^{C_{p}\left(1+\sup _{0 \leq s \leq t}\left\|\nabla^{2} \phi_{f}(s)\right\|_{\infty}\right) t}\left\{\left\|\mid w_{\tilde{\vartheta}} f(0)\right\|_{p}^{p}+\left\|w_{\tilde{\vartheta}} \alpha_{f, \varepsilon}^{\beta} \partial f(0)\right\|_{p}^{p}\right\} .
\end{aligned}
$$

Lemma 11. For any $0<\delta<1$, we claim that if $\left(f, \phi_{f}\right)$ solves (1.16) then

$$
\left\|\phi_{f}(t)\right\|_{C^{1,1-\delta}(\bar{\Omega})} \leq C_{\Omega}\left\|w_{\vartheta} f(t)\right\|_{\infty} \text { for all } t \geq 0 .
$$

Proof. We have, for any $p>1$,

$$
\left\|\int_{\mathbb{R}^{3}} f(t, x, v) \sqrt{\mu(v)} \mathrm{d} v\right\|_{L^{p}(\Omega)} \leq|\Omega|^{1 / p}\left(\int_{\mathbb{R}^{3}} w_{\vartheta}(v)^{-1} \sqrt{\mu(v)} \mathrm{d} v\right)\left\|w_{\vartheta} f(t)\right\|_{\infty} .
$$

Then we apply the standard elliptic estimate to (1.16) and deduce that

$$
\left\|\phi_{f}(t)\right\|_{W^{2, p}(\Omega)} \lesssim\left\|w_{\vartheta} f(t)\right\|_{\infty}
$$

On the other hand, from the Morrey inequality, we have, for $p>3$ and $\Omega \subset \mathbb{R}^{3}$,

$$
\left\|\phi_{f}(t)\right\|_{C^{1,1-3 / p}(\Omega)} \lesssim_{p, \Omega}\left\|\phi_{f}(t)\right\|_{W^{2, p}(\Omega)} .
$$

Now we choose $p=3 / \delta$ for $0<\delta<1$. Then we can obtain (4.6). Note that the constant $C_{\Omega}$ can be chosen independent of $p$ and $\delta$.

To close the estimate, we use the following lemma crucially.

Lemma 12. Assume [4.1). If $\phi_{f}$ solves (1.16) then there exists $C_{1} \geq 0$ such that

$$
\left\|\phi_{f}(t)\right\|_{C^{2,1-\frac{3}{p}}(\bar{\Omega})} \leq\left(C_{1}\right)^{1 / p}\left\{\|f(t)\|_{p}+\left\|\alpha_{f, \varepsilon}^{\beta} \nabla_{x} f(t)\right\|_{p}\right\} .
$$

Proof. Applying the Schauder estimate to (1.16), we deduce

$$
\left\|\phi_{f}(t)\right\|_{C^{2,1-\frac{3}{p}}(\bar{\Omega})} \lesssim p, \Omega\left\|\int_{\mathbb{R}^{3}} f(t) \sqrt{\mu} \mathrm{d} v\right\|_{C^{0,1-\frac{3}{p}(\bar{\Omega})}} \text { for } p>3 .
$$

By the Morrey inequality, $W^{1, p} \subset C^{0,1-\frac{3}{p}}$ with $p>3$ for a domain $\Omega \subset \mathbb{R}^{3}$ with a smooth boundary $\partial \Omega$, we derive

$$
\begin{aligned}
& \left\|\int_{\mathbb{R}^{3}} f(t) \sqrt{\mu} \mathrm{d} v\right\|_{C^{0,1-\frac{n}{p}}(\bar{\Omega})} \\
\lesssim & \left\|\int_{\mathbb{R}^{3}} f(t) \sqrt{\mu} \mathrm{d} v\right\|_{W^{1, p}(\Omega)} \\
\lesssim & \left(\int_{\mathbb{R}^{3}} \mu^{q / 2} \mathrm{~d} v\right)^{1 / q}\|f(t)\|_{L^{p}\left(\Omega \times \mathbb{R}^{3}\right)}+\left\|\int_{\mathbb{R}^{3}} \nabla_{x} f(t) \sqrt{\mu} \mathrm{d} v\right\|_{L^{p}(\Omega)} .
\end{aligned}
$$

By the Hölder inequality

$$
\begin{aligned}
& \left|\int_{\mathbb{R}^{3}} \nabla_{x} f(t, x, v) \sqrt{\mu(v)} \mathrm{d} v\right| \\
& \leq\left\|\frac{\sqrt{\mu(\cdot)}}{\alpha_{f, \varepsilon}(t, x, \cdot)^{\beta}}\right\|_{L^{\frac{p}{p-1}\left(\mathbb{R}^{3}\right)}}\left\|\alpha_{f, \varepsilon}(t, x, \cdot)^{\beta} \nabla_{x} f(t, x, \cdot)\right\|_{L^{p}\left(\mathbb{R}^{3}\right)} \\
& =\underbrace{\left(\int_{\mathbb{R}_{1}} \frac{\mu(v)^{\frac{p}{2(p-1)}}}{\alpha_{f, \varepsilon}(t, x, v)^{\frac{\beta p}{p-1}}} \mathrm{~d} v\right)^{\frac{p-1}{p}}}_{\underline{\mathbb{R}}^{3} 10}\left\|\alpha_{f, \varepsilon}(t, x, \cdot)^{\beta} \nabla_{x} f(t, x, \cdot)\right\|_{L^{p}\left(\mathbb{R}^{3}\right)} .
\end{aligned}
$$

Note that $\frac{p-2}{p-1}<\frac{\beta p}{p-1}<\frac{2}{3} \frac{p}{p-1}<1$ from (4.1). We apply Proposition 3 and conclude that $4.101 \lesssim 1$. Taking $L^{p}(\Omega)$-norm on (4.10) and from (4.9), we conclude (4.7). 
We need some basic estimates to prove Proposition 4. Recall the decomposition of $L$ in (2.1). From (2.2)

$$
\left|\nabla_{v} \nu(v)\right| \leq \int_{\mathbb{R}^{3}} \int_{\mathbb{S}^{2}}|\omega| \mu(u) \mathrm{d} \omega \mathrm{d} u \lesssim 1
$$

We define a notation

$$
\mathbf{k}_{\varrho}(v, u):=\frac{1}{|v-u|} \exp \left\{-\varrho|v-u|^{2}-\varrho \frac{\left.|| v\right|^{2}-\left.|u|^{2}\right|^{2}}{|v-u|^{2}}\right\} .
$$

It is standard (see [17]) that for $0<\frac{\vartheta}{4}<\varrho$ and $0<\tilde{\varrho}<\varrho-\frac{\vartheta}{4}$,

$$
\mathbf{k}_{\varrho}(v, u) e^{\vartheta|v|^{2}} e^{-\vartheta|u|^{2}} \lesssim \mathbf{k}_{\tilde{\varrho}}(v, u)
$$

Moreover, for $0<\frac{\vartheta}{4}<\varrho$, (see the proof of Lemma 7 in [17])

$$
\int_{\mathbb{R}^{3}} \mathbf{k}_{\varrho}(v, u) e^{\vartheta|v|^{2}} e^{-\vartheta|u|^{2}} \mathrm{~d} u \lesssim\langle v\rangle^{-1}
$$

From (2.4) and a direct computation, for $0<\varrho<\frac{1}{8}$,

$$
\left|\partial_{v_{i}} \mathbf{k}_{1}(v, u+v)\right|=C_{\mathbf{k}_{1}} \partial_{v_{i}}\left(|u| e^{-\frac{|v|^{2}+|u+v|^{2}}{4}}\right) \lesssim \mathbf{k}_{\varrho}(v, u+v)
$$

and

$$
\begin{aligned}
\partial_{v_{i}} \mathbf{k}_{2}(v, u+v) & =C_{\mathbf{k}_{2}} \partial_{v_{i}}\left(\frac{1}{|u|} e^{-\frac{|u|^{2}}{8}} e^{-\frac{\left.|| v\right|^{2}-\left.|u+v|^{2}\right|^{2}}{8|u|^{2}}}\right) \\
& =-\frac{C_{\mathbf{k}_{2}}}{|u|} e^{-\frac{|u|^{2}}{8}} e^{-\frac{\left.|| v\right|^{2}-\left.|u+v|^{2}\right|^{2}}{8|u|^{2}}} \frac{\left.|| v\right|^{2}-|u+v|^{2} \mid}{|u|} \frac{u_{i}}{|u|} \\
& \lesssim|u|^{-1} e^{-\frac{|u|^{2}}{8}} e^{-\frac{\left.|| v\right|^{2}-\left.|u+v|^{2}\right|^{2}}{16|u|^{2}}} \\
& \lesssim \mathbf{k}_{\varrho}(v, u+v) .
\end{aligned}
$$

We define

$$
K_{v} g(v):=\int_{\mathbb{R}^{3}}\left\{\nabla_{v} \mathbf{k}_{2}(v, u+v)-\nabla_{v} \mathbf{k}_{1}(v, u+v)\right\} g(u+v) \mathrm{d} u .
$$

From (4.13), 4.15), and 4.16),

$$
\begin{aligned}
\left|w_{\tilde{\vartheta}} K \nabla_{v} g(v)\right| & \left.\leq \sum_{i} \int_{\mathbb{R}^{3}}\left|\mathbf{k}_{i}(v, u+v)\right| \frac{w_{\tilde{\vartheta}}(v)}{w_{\tilde{\vartheta}}(u+v)}\left|w_{\tilde{\vartheta}} \nabla_{v} g(u+v)\right|\right\} \mathrm{d} u \\
& \lesssim \int_{\mathbb{R}^{3}} \mathbf{k}_{\tilde{\varrho}}(v, u)\left|w_{\tilde{\vartheta}} \nabla_{v} g(u)\right| \mathrm{d} u \\
\left|w_{\tilde{\vartheta}} K_{v} g(v)\right| & \leq \sum_{i} \int_{\mathbb{R}^{3}}\left|\nabla_{v} \mathbf{k}_{i}(v, u+v)\right| \frac{w_{\tilde{\vartheta}}(v)}{w_{\vartheta}(u+v)}\left|w_{\vartheta} g(u+v)\right| \mathrm{d} u \\
& \lesssim \int_{\mathbb{R}^{3}} \mathbf{k}_{\varrho}(v, u) \frac{w_{\tilde{\vartheta}}(v)}{w_{\vartheta}(u)}\left|w_{\vartheta} g(u)\right| \mathrm{d} u \\
& \lesssim\left\|w_{\vartheta} g\right\|_{\infty} .
\end{aligned}
$$

The nonlinear Boltzmann operator (2.5) equals

$$
\begin{aligned}
& \int_{\mathbb{R}^{3}} \int_{\mathbb{S}^{2}}|u \cdot \omega| g_{1}\left(v+u_{\perp}\right) g_{2}\left(v+u_{\|}\right) \sqrt{\mu(v+u)} \mathrm{d} \omega \mathrm{d} u \\
& -\int_{\mathbb{R}^{3}} \int_{\mathbb{S}^{2}}|u \cdot \omega| g_{1}(v+u) g_{2}(v) \sqrt{\mu(v+u)} \mathrm{d} \omega \mathrm{d} u,
\end{aligned}
$$

where $u_{\|}=(u \cdot \omega) \omega$ and $u_{\perp}=u-u_{\|}$. Following the derivation of (2.4) in Chapter 3 of [20], by exchanging the role of $\sqrt{\mu}$ and $w^{-1}$, we have

$$
\left|w_{\vartheta} \Gamma(g, g)\right| \lesssim\left\|w_{\vartheta} g\right\|_{\infty} \int_{\mathbb{R}^{3}} \mathbf{k}_{\tilde{\varrho}}(v, u)\left|w_{\vartheta} g(u)\right| \mathrm{d} u
$$


By direct computations

$$
\begin{aligned}
& \nabla_{v} \Gamma(g, g)(v) \\
= & \nabla_{v} \Gamma_{\text {gain }}(g, g)-\nabla_{v} \Gamma_{\text {loss }}(g, g) \\
= & \Gamma_{\text {gain }}\left(\nabla_{v} g, g\right)+\Gamma_{\text {gain }}\left(g, \nabla_{v} g\right) \\
& -\Gamma_{\text {loss }}\left(\nabla_{v} g, g\right)-\Gamma_{\text {loss }}\left(g, \nabla_{v} g\right)+\Gamma_{v}(g, g) .
\end{aligned}
$$

Here we have defined

$$
\begin{aligned}
\Gamma_{v}\left(g_{1}, g_{2}\right)(v):= & \Gamma_{v, \text { gain }}-\Gamma_{v, \text { loss }} \\
:= & \int_{\mathbb{R}^{3}} \int_{\mathbb{S}^{2}}|u \cdot \omega| g_{1}\left(v+u_{\perp}\right) g_{2}\left(v+u_{\|}\right) \nabla_{v} \sqrt{\mu(v+u)} \mathrm{d} \omega \mathrm{d} u \\
& -\int_{\mathbb{R}^{3}} \int_{\mathbb{S}^{2}}|u \cdot \omega| g_{1}(v+u) g_{2}(v) \nabla_{v} \sqrt{\mu(v+u)} \mathrm{d} \omega \mathrm{d} u .
\end{aligned}
$$

Note that

$$
\begin{aligned}
& \left|w_{\tilde{\vartheta}} \Gamma_{\text {gain }}\left(\nabla_{v} g, g\right)\right|+\left|w_{\tilde{\vartheta}} \Gamma_{\text {gain }}\left(g, \nabla_{v} g\right)\right| \\
\lesssim & \left\|w_{\vartheta} g\right\|_{\infty}\left\{\left|w_{\tilde{\vartheta}} \Gamma_{\text {gain }}\left(\left|\nabla_{v} g\right|, w_{\vartheta}^{-1}\right)\right|+\left|w_{\tilde{\vartheta}} \Gamma_{\text {gain }}\left(w_{\vartheta}^{-1},\left|\nabla_{v} g\right|\right)\right|\right\} \\
\lesssim & \left\|w_{\vartheta} g\right\|_{\infty} \int_{\mathbb{R}^{3}} \int_{\mathbb{S}^{2}}|(v-u) \cdot \omega| \frac{w_{\tilde{\vartheta}}(v)}{w_{\vartheta}(u)}\left\{\frac{\left|\nabla_{v} g\left(u^{\prime}\right)\right|}{w_{\vartheta}\left(v^{\prime}\right)}+\frac{\left|\nabla_{v} g\left(v^{\prime}\right)\right|}{w_{\vartheta}\left(u^{\prime}\right)}\right\} \mathrm{d} \omega \mathrm{d} u .
\end{aligned}
$$

Then following the derivation of (2.4) in Chapter 3 of [20], by exchanging the role of $\sqrt{\mu}$ and $w_{\vartheta}^{-1}$, we can obtain a bound of

$$
\begin{aligned}
& \left|w_{\tilde{\vartheta}} \Gamma_{\text {gain }}\left(\nabla_{v} g, g\right)\right|+\left|w_{\tilde{\vartheta}} \Gamma_{\text {gain }}\left(g, \nabla_{v} g\right)\right| \\
\lesssim & \left\|w_{\vartheta} g\right\|_{\infty} \int_{\mathbb{R}^{3}} \mathbf{k}_{\varrho}(v, u) \frac{w_{\tilde{\vartheta}}(v)}{w_{\tilde{\vartheta}}(u)}\left|w_{\tilde{\vartheta}} \nabla_{v} g(u)\right| \mathrm{d} u \\
\lesssim & \left\|w_{\vartheta} g\right\|_{\infty} \int_{\mathbb{R}^{3}} \mathbf{k}_{\tilde{\varrho}}(v, u)\left|w_{\tilde{\vartheta}} \nabla_{v} g(u)\right| \mathrm{d} u .
\end{aligned}
$$

Clearly

$$
\begin{aligned}
\left|w_{\tilde{\vartheta}} \Gamma_{\text {loss }}\left(\nabla_{v} g, g\right)\right| & \lesssim\left\|w_{\vartheta} g\right\|_{\infty} \int_{\mathbb{R}^{3}} \frac{w_{\tilde{\vartheta}}(v)}{w_{\vartheta}(v) w_{\tilde{\vartheta}}(u)}\left|w_{\tilde{\vartheta}} \nabla_{v} g(u)\right| \mu(u)^{\frac{1}{2}} \mathrm{~d} u \\
& \lesssim\left\|w_{\vartheta} g\right\|_{\infty} \int_{\mathbb{R}^{3}} \mathbf{k}_{\tilde{\varrho}}(v, u)\left|w_{\tilde{\vartheta}} \nabla_{v} g(u)\right| \mathrm{d} u \\
\left|w_{\tilde{\vartheta}} \Gamma_{\text {loss }}\left(g, \nabla_{v} g\right)\right| & \lesssim\langle v\rangle\left\|w_{\vartheta} g\right\|_{\infty}\left|w_{\tilde{\vartheta}} \nabla_{v} g(v)\right| .
\end{aligned}
$$

For $\Gamma_{v, \operatorname{loss}}(g, g)$ defined in (4.22),

$$
\begin{aligned}
& \left|w_{\tilde{\vartheta}} \Gamma_{v, \text { loss }}(g, g)\right| \\
& \lesssim \frac{w_{\tilde{\vartheta}}(v)}{w_{\vartheta}(v)}\left\|w_{\vartheta} g\right\|_{\infty} \iint_{\mathbb{R}^{3} \times \mathbb{S}^{2}}|(u-v) \cdot \omega| \frac{1}{w_{\vartheta}(u)}\left|w_{\vartheta} g(u)\right| \nabla_{v} \sqrt{\mu(u)} \mathrm{d} u \mathrm{~d} \omega \\
& \lesssim\langle v\rangle\left\|w_{\vartheta} g\right\|_{\infty}^{2} .
\end{aligned}
$$

For $\Gamma_{v, \text { gain }}(g, g)$, following the derivation of (2.4) in Chapter 3 of [20], by exchanging the role of $\sqrt{\mu}$ and $w_{\vartheta}^{-1}$

$$
\begin{aligned}
& \left|w_{\tilde{\vartheta}} \Gamma_{v, \text { gain }}(g, g)\right| \\
\lesssim & \left\|w_{\vartheta} g\right\|_{\infty} \iint_{\mathbb{R}^{3} \times \mathbb{S}^{2}}|(u-v) \cdot \omega| \frac{w_{\tilde{\vartheta}}(v)}{w_{\vartheta}\left(v^{\prime}\right)} \frac{w_{\vartheta} g\left(v^{\prime}\right)}{w_{\vartheta}\left(u^{\prime}\right)} \nabla_{v} \sqrt{\mu(u)} \mathrm{d} u \mathrm{~d} \omega \\
\lesssim & \left\|w_{\vartheta} g\right\|_{\infty} \int_{\mathbb{R}^{3}} \mathbf{k}_{\tilde{\varrho}}(v, u)\left|w_{\vartheta} g(u)\right| \mathrm{d} u .
\end{aligned}
$$

The next result is about estimates of derivatives on the boundary. Assume (1.15) and (1.17). We claim that for $(x, v) \in \gamma_{-}$,

$$
\left|\nabla_{x, v} f(t, x, v)\right| \lesssim\langle v\rangle \sqrt{\mu(v)}\left(1+\frac{1}{|n(x) \cdot v|}\right) \times \text { (4.28) }
$$


with

$$
\begin{aligned}
\int_{n(x) \cdot u>0}\{ & \left(\langle u\rangle+\left|\nabla_{x} \phi_{f}\right|\right)\left|\nabla_{x, v} f(t, x, u)\right| \\
& +\langle u\rangle|f|+\left(1+\left\|w_{\vartheta} f\right\|_{\infty}\right)\left|\int_{\mathbb{R}^{3}} \mathbf{k}_{\varrho}\left(u, u^{\prime}\right)\right| f\left(u^{\prime}\right)\left|\mathrm{d} u^{\prime}\right| \\
& \left.+\left(\langle u\rangle|f|+\mu(u)^{\frac{1}{4}}\right)\left|\nabla_{x} \phi_{f}\right|\right\} \sqrt{\mu(u)}\{n(x) \cdot u\} \mathrm{d} u .
\end{aligned}
$$

From (1.15),

$$
\begin{aligned}
& \partial_{n} f(t, x, v) \\
& \begin{aligned}
\frac{-1}{n(x) \cdot v} & \left\{\partial_{t} f+\sum_{i=1}^{2}\left(v \cdot \tau_{i}\right) \partial_{\tau_{i}} f-\nabla_{x} \phi_{f} \cdot \nabla_{v} f\right. \\
& \left.\quad+\frac{v}{2} \cdot \nabla_{x} \phi_{f} f+L f-\Gamma(f, f)+v \cdot \nabla_{x} \phi_{f} \sqrt{\mu}\right\} .
\end{aligned}
\end{aligned}
$$

Let $\tau_{1}(x)$ and $\tau_{2}(x)$ be unit tangential vectors to $\partial \Omega$ satisfying $\tau_{1}(x) \cdot n(x)=0=\tau_{2}(x) \cdot n(x)$ and $\tau_{1}(x) \times$ $\tau_{2}(x)=n(x)$. Define the orthonormal transformation from $\left\{n, \tau_{1}, \tau_{2}\right\}$ to the standard basis $\left\{\mathbf{e}_{1}, \mathbf{e}_{2}, \mathbf{e}_{3}\right\}$, i.e. $\mathcal{T}(x) n(x)=\mathbf{e}_{1}, \mathcal{T}(x) \tau_{1}(x)=\mathbf{e}_{2}, \mathcal{T}(x) \tau_{2}(x)=\mathbf{e}_{3}$, and $\mathcal{T}^{-1}=\mathcal{T}^{T}$. Upon a change of variable: $u^{\prime}=\mathcal{T}(x) u$, we have

$$
n(x) \cdot u=n(x) \cdot \mathcal{T}^{t}(x) u^{\prime}=n(x)^{t} \mathcal{T}^{t}(x) u^{\prime}=[\mathcal{T}(x) n(x)]^{t} u^{\prime}=\mathbf{e}_{1} \cdot u^{\prime}=u_{1}^{\prime},
$$

then the RHS of the diffuse BC (1.17) equals

$$
c_{\mu} \sqrt{\mu(v)} \int_{u_{1}^{\prime}>0} f\left(t, x, \mathcal{T}^{t}(x) u^{\prime}\right) \sqrt{\mu\left(u^{\prime}\right)}\left\{u_{1}^{\prime}\right\} \mathrm{d} u^{\prime} .
$$

Then we can further take tangential derivatives $\partial_{\tau_{i}}$ as, for $(x, v) \in \gamma_{-}$,

$$
\begin{aligned}
& \partial_{\tau_{i}} f(t, x, v) \\
& =c_{\mu} \sqrt{\mu(v)} \int_{n(x) \cdot u>0} \partial_{\tau_{i}} f(t, x, u) \sqrt{\mu(u)}\{n(x) \cdot u\} \mathrm{d} u \\
& \quad+c_{\mu} \sqrt{\mu(v)} \int_{n(x) \cdot u>0} \nabla_{v} f(t, x, u) \frac{\partial \mathcal{T}^{t}(x)}{\partial \tau_{i}} \mathcal{T}(x) u \sqrt{\mu(u)}\{n(x) \cdot u\} \mathrm{d} u .
\end{aligned}
$$

We can take velocity derivatives directly to (1.17) and obtain that for $(x, v) \in \gamma_{-}$,

$$
\begin{aligned}
\nabla_{v} f(t, x, v) & =c_{\mu} \nabla_{v} \sqrt{\mu(v)} \int_{n(x) \cdot u>0} f(t, x, u) \sqrt{\mu(u)}\{n(x) \cdot u\} \mathrm{d} u, \\
\partial_{t} f(t, x, v) & =c_{\mu} \sqrt{\mu(v)} \int_{n(x) \cdot u>0} \partial_{t} f(t, x, u) \sqrt{\mu(u)}\{n(x) \cdot u\} \mathrm{d} u .
\end{aligned}
$$

For the temporal derivative, we use (1.15) again to deduce that

$$
\begin{aligned}
& \partial_{t} f(t, x, v) \\
=c_{\mu} \sqrt{\mu(v)} \int_{n(x) \cdot u>0}\{ & -u \cdot \nabla_{x} f+\nabla_{x} \phi \cdot \nabla_{v} f-\frac{u}{2} \cdot \nabla_{x} \phi f-L f \\
& \left.+\Gamma(f, f)-u \cdot \nabla_{x} \phi \sqrt{\mu}\right\} \sqrt{\mu(u)}\{n(x) \cdot u\} \mathrm{d} u .
\end{aligned}
$$

From (4.29)-(4.32), (2.4), and (4.20), we conclude (4.27).

Proof of Proposition 4. Step 1. Define

$$
\nu_{\phi_{f}}(t, x, v):=\nu(v)+\frac{v}{2} \cdot \nabla_{x} \phi_{f} .
$$

From the assumption (4.2), we have that $\nu_{\phi_{f}}(t, x, v) \gtrsim \frac{\nu(v)}{2}$. 
From (1.15), (2.4), and (4.20), we can easily obtain that, for $0<\varrho \ll 1$

$$
\begin{aligned}
& \left\|w_{\tilde{\vartheta}} f(t)\right\|_{p}^{p}+\int_{0}^{t}\left\|\nu_{\phi_{f}}^{1 / p} w_{\tilde{\vartheta}} f\right\|_{p}^{p}+\int_{0}^{t}\left|w_{\tilde{\vartheta}} f\right|_{p,+}^{p} \\
& \lesssim\left\|w_{\tilde{\vartheta}} f(0)\right\|_{p}^{p}+\left(1+\left\|w_{\vartheta} f\right\|_{\infty}\right) \int_{0}^{t} \iint_{\Omega \times \mathbb{R}^{3}}\left|w_{\tilde{\vartheta}} f(v)\right|^{p-1} \\
& \times \int_{\mathbb{R}^{3}} \mathbf{k}_{\varrho}(v, u) \frac{w_{\tilde{\vartheta}}(v)}{w_{\tilde{\vartheta}}(u)}\left|w_{\tilde{\vartheta}} f(u)\right| \mathrm{d} u \\
& +\int_{0}^{t}\left\|w_{\tilde{\vartheta}} f\right\|_{p}^{p}+\int_{0}^{t}\left\|w_{\tilde{\vartheta}} \nabla \phi_{f}\right\|_{p}^{p}+\int_{0}^{t}\left|w_{\tilde{\vartheta}} f\right|_{p,-}^{p} .
\end{aligned}
$$

Note that by the Hölder inequality, 4.14), and (4.13),

$$
\begin{aligned}
& \int_{\mathbb{R}^{3}}\left|w_{\tilde{\vartheta}} f(v)\right|^{p-1} \int_{\mathbb{R}^{3}} \mathbf{k}_{\tilde{\varrho}}(v, u)\left|w_{\tilde{\vartheta}} f(u)\right| \mathrm{d} u \mathrm{~d} v \\
\lesssim & \left\|w_{\tilde{\vartheta}} f\right\|_{L_{v}^{p}}^{\frac{1}{p-1}}\left\|\int_{\mathbb{R}^{3}} \mathbf{k}_{\tilde{\varrho}}(v, u)^{1 / q} \mathbf{k}_{\tilde{\varrho}}(v, u)^{1 / p}\left|w_{\tilde{\vartheta}} f(u)\right| \mathrm{d} u\right\|_{L_{v}^{p}} \\
\lesssim & \left\|w_{\tilde{\vartheta}} f\right\|_{L_{v}^{p}}^{\frac{1}{p-1}}\left(\int_{\mathbb{R}^{3}} \mathbf{k}_{\tilde{\varrho}}(v, u) \mathrm{d} u\right)^{1 / q}\left\|\left(\int_{\mathbb{R}^{3}} \mathbf{k}_{\tilde{\varrho}}(v, u)\left|w_{\tilde{\vartheta}} f(u)\right|^{p} \mathrm{~d} u\right)^{1 / p}\right\|_{L_{v}^{p}} \\
\lesssim & \left\|w_{\tilde{\vartheta}} f\right\|_{L_{v}^{p}}^{p}\left(\int_{\mathbb{R}^{3}} \mathbf{k}_{\tilde{\varrho}}(v, u) \mathrm{d} u\right)^{1 / q}\left(\int_{\mathbb{R}^{3}} \mathbf{k}_{\tilde{\varrho}}(v, u) \mathrm{d} v\right)^{1 / p} \\
\lesssim & \left\|w_{\tilde{\vartheta}} f\right\|_{L_{v}^{p}}^{p} .
\end{aligned}
$$

From a standard elliptic theorem and (1.16), we have

$$
\int_{0}^{t}\left\|\nabla \phi_{f}\right\|_{p}^{p} \lesssim \int_{0}^{t}\left\|w_{\tilde{\vartheta}} f\right\|_{p}^{p} .
$$

Now we focus on $\int_{0}^{t}\left|w_{\tilde{\vartheta}} f\right|_{p,-}^{p}$ in (4.34). We plug in (1.17) and then decompose $\gamma_{+}^{\varepsilon} \cup \gamma_{+} \backslash \gamma_{+}^{\varepsilon}$ where $\varepsilon$ is small but satisfies (2.51). This leads

$$
\begin{aligned}
& \int_{0}^{t}\left|w_{\tilde{\vartheta}} f\right|_{p,-}^{p} \\
\lesssim & \int_{0}^{t} \int_{\partial \Omega}\left(\int_{\gamma_{+}^{\varepsilon}(x)} w_{\tilde{\vartheta}} f \sqrt{\mu}\{n \cdot u\} \mathrm{d} u\right)^{p} \\
& +\int_{0}^{t} \int_{\partial \Omega}\left(\int_{\gamma_{+}(x) \backslash \gamma_{+}^{\varepsilon}(x)} w_{\tilde{\vartheta}} f \sqrt{\mu}\{n \cdot u\} \mathrm{d} u\right)^{p} \\
\lesssim & \left(\int_{\gamma_{+}^{\varepsilon}} \sqrt{\mu}\{n \cdot u\} \mathrm{d} u\right)^{p / q} \int_{0}^{t}\left|w_{\tilde{\vartheta}} f\right|_{p,+}^{p}+\int_{0}^{t} \int_{\gamma_{+} \backslash \gamma_{+}^{\varepsilon}}\left|w_{\tilde{\vartheta}} f \sqrt{\mu}\right|^{p} \\
\lesssim & o(1) \int_{0}^{t}\left|w_{\tilde{\vartheta}} f\right|_{p,+}^{p}+\int_{0}^{t} \int_{\gamma_{+} \backslash \gamma_{+}^{\varepsilon}}\left|w_{\tilde{\vartheta}} f \sqrt{\mu}\right|^{p} .
\end{aligned}
$$

From (1.15), Lemma 7. (2.4), 4.20), and 4.35)

$$
\begin{aligned}
\int_{0}^{t}\left|w_{\tilde{\vartheta}} f\right|_{p,-}^{p} \lesssim & \left\|w_{\tilde{\vartheta}} f(0)\right\|_{p}^{p}+o(1) \int_{0}^{t}\left|w_{\tilde{\vartheta}} f\right|_{p,+}^{p} \\
& +\left(1+\left\|w_{\vartheta} f\right\|_{\infty}\right) \int_{0}^{t}\left\|w_{\tilde{\vartheta}} f\right\|_{p}^{p} .
\end{aligned}
$$

Collecting terms from (4.34), 4.35), 4.36), and (4.37), we conclude that

$$
\begin{aligned}
& \left\|w_{\tilde{\vartheta}} f(t)\right\|_{p}^{p}+\int_{0}^{t}\left\|\nu_{\phi_{f}}^{1 / p} w_{\tilde{\vartheta}} f\right\|_{p}^{p}+\int_{0}^{t}\left|w_{\tilde{\vartheta}} f\right|_{p,+}^{p} \\
\lesssim & \left\|w_{\tilde{\vartheta}} f(0)\right\|_{p}^{p}+\left(1+\left\|w_{\vartheta} f\right\|_{\infty}\right) \int_{0}^{t}\left\|w_{\tilde{\vartheta}} f\right\|_{p}^{p} .
\end{aligned}
$$


Step 2. By taking derivatives $\partial \in\left\{\nabla_{x_{i}}, \nabla_{v_{i}}\right\}$ to (1.15),

$$
\left[\partial_{t}+v \cdot \nabla_{x}-\nabla_{x} \phi_{f} \cdot \nabla_{v}+\nu_{\phi_{f}, w_{\tilde{\vartheta}}}\right]\left(w_{\tilde{\vartheta}} \partial f\right)=w_{\tilde{\vartheta}} \mathcal{G}
$$

where

$$
\begin{aligned}
\mathcal{G}= & -\partial v \cdot \nabla_{x} f+\partial \nabla \phi_{f} \cdot \nabla_{v} f \\
& +\partial \Gamma(f, f)-\partial\left[\nu(v)+\frac{v}{2} \cdot \nabla \phi_{f}(t, x)\right] f-\partial K f-\partial\left(v \cdot \nabla_{x} \phi_{f} \sqrt{\mu}\right) .
\end{aligned}
$$

Here we have used

$$
\nu_{\phi_{f}, w_{\tilde{\vartheta}}}=\nu_{\phi_{f}, w_{\tilde{\vartheta}}}(t, x, v):=\nu(v)+\frac{v}{2} \cdot \nabla \phi_{f}(t, x)+\frac{\nabla_{x} \phi_{f} \cdot \nabla_{v} w_{\tilde{\vartheta}}}{w_{\tilde{\vartheta}}} .
$$

From (1.28) and (4.39),

$$
\begin{aligned}
& \frac{1}{p}\left|w_{\tilde{\vartheta}} \alpha_{f, \varepsilon}^{\beta} \partial f\right|^{p-1}\left[\partial_{t}+v \cdot \nabla_{x}-\nabla_{x} \phi_{f} \cdot \nabla_{v}+\nu_{\phi_{f}, w_{\tilde{\vartheta}}}\right]\left|w_{\tilde{\vartheta}} \alpha_{f, \varepsilon}^{\beta} \partial f\right| \\
& =\alpha_{f, \varepsilon}^{\beta p}\left|w_{\tilde{\vartheta}} \partial f\right|^{p-1}\left[\partial_{t}+v \cdot \nabla_{x}-\nabla_{x} \phi_{f} \cdot \nabla_{v}+\nu_{\phi_{f}, w_{\tilde{\vartheta}}}\right]\left|w_{\tilde{\vartheta}} \partial f\right| \\
& =w_{\tilde{\vartheta}}^{p} \alpha_{f, \varepsilon}^{\beta p}|\partial f|^{p-1} \mathcal{G} .
\end{aligned}
$$

From (4.11), 4.41), 4.17), and 4.22)

$$
\begin{aligned}
|\mathcal{G}| \lesssim & \left|\nabla_{x} f\right|+\left|\nabla^{2} \phi_{f}\right|\left|\nabla_{v} f\right|+|\Gamma(\partial f, f)|+|\Gamma(f, \partial f)|+|K \partial f| \\
& +|f|+\left|\Gamma_{v}(f, f)\right|+\left|K_{v} f\right| \\
& +w_{\vartheta}(v)^{-1 / 2}\left(\left|\nabla \phi_{f}\right|+\left|\nabla^{2} \phi_{f}\right|\right)\left(1+\left\|w_{\vartheta} f\right\|_{\infty}\right) .
\end{aligned}
$$

Now we apply Lemma 6 to (4.42) to obtain

$$
\begin{aligned}
& \left\|w_{\tilde{\vartheta}} \alpha_{f, \varepsilon}^{\beta} \partial f(t)\right\|_{p}^{p}+\int_{0}^{t}\left\|\nu_{\phi_{f}, w_{\tilde{\vartheta}}}^{1 / p} w_{\tilde{\vartheta}} \alpha_{f, \varepsilon}^{\beta} \partial f\right\|_{p}^{p}+\int_{0}^{t}\left|w_{\tilde{\vartheta}} \alpha_{f, \varepsilon}^{\beta} \partial f\right|_{p,+}^{p} \\
& \leq\left\|w_{\tilde{\vartheta}} \alpha_{f, \varepsilon}^{\beta} \partial f(0)\right\|_{p}^{p}+\underbrace{\int_{0}^{t}\left|w_{\tilde{\vartheta}} \alpha_{f, \varepsilon}^{\beta} \partial f\right|_{p,-}^{p}} \\
& 4.44 \gamma_{-} \\
& +\underbrace{\int_{0}^{t} \iint_{\Omega \times \mathbb{R}^{3}} p \alpha_{f, \varepsilon}^{\beta p} w_{\tilde{\vartheta}}^{p}|\partial f|^{p-1}|\mathcal{G}|}_{\text {4.44 }_{\mathcal{G}}} .
\end{aligned}
$$
by

First we consider (4.44) $\mathcal{G}$. Directly, the contribution of $\left|\nabla_{x} f\right|+\left|\nabla^{2} \phi_{f}\right|\left|\nabla_{v} f\right|$ of (4.43) in (4.44) $\mathcal{G}$ is bounded

$$
\left(1+\sup _{0 \leq s \leq t}\left\|\nabla^{2} \phi_{f}\right\|_{\infty}\right) \int_{0}^{t}\left\|w_{\tilde{\vartheta}} \alpha_{f, \varepsilon}^{\beta} \partial f\right\|_{p}^{p} .
$$

From (4.18), (4.23), and (4.24), the contribution of $|\Gamma(\partial f, f)|+\left|\Gamma_{\text {gain }}(f, \partial f)\right|+|K \partial f|$ of (4.43) in (4.44) $\mathcal{G}$ is bounded by

$$
\begin{aligned}
& \left(1+\sup _{0 \leq s \leq t}\left\|w_{\vartheta} f(s)\right\|_{\infty}\right) \\
& \times \int_{0}^{t} \iint_{\Omega \times \mathbb{R}^{3}}\left|\alpha_{f, \varepsilon}^{\beta} w_{\tilde{\vartheta}} \partial f(v)\right|^{p-1} \\
& \times \int_{\mathbb{R}^{3}} \alpha_{f, \varepsilon}(v)^{\beta} \mathbf{k}_{\varrho}(v, u) w_{\tilde{\vartheta}}(v)|\partial f(u)| \mathrm{d} u \mathrm{~d} v \mathrm{~d} x \mathrm{~d} s .
\end{aligned}
$$

The estimate of (4.46) is carried out in Step 3.

From (4.24), the contribution of $\left|\Gamma_{\text {loss }}(f, \partial f)\right|$ of (4.43) in (4.44) $\mathcal{G}$ is bounded by

$$
\sup _{0 \leq s \leq t}\left\|w_{\vartheta} f(s)\right\|_{\infty} \int_{0}^{t}\left\|\nu_{\phi_{f}, w_{\tilde{\vartheta}}}^{1 / p} w_{\tilde{\vartheta}} \alpha_{f, \varepsilon}^{\beta} \partial f\right\|_{p}^{p} .
$$


For the $|f|$ contribution of (4.43) in (4.44) $\mathcal{G}$, we bound

$$
\begin{aligned}
& \int_{0}^{t} \iint_{\Omega \times \mathbb{R}^{3}} p w_{\tilde{\vartheta}}^{p} \alpha_{f, \varepsilon}^{\beta p}|\partial f|^{p-1}|f| \mathrm{d} x \mathrm{~d} v \mathrm{~d} s \\
\lesssim & \int_{0}^{t} \iint_{\Omega \times \mathbb{R}^{3}}\left|\nu_{\phi_{f}, w_{\tilde{\vartheta}}}^{1 / p} w_{\tilde{\vartheta}} \alpha_{f, \varepsilon}^{\beta} \partial f\right|^{p-1}\left|w_{\tilde{\vartheta}} f\right| \frac{\alpha_{f, \varepsilon}(s, x, v)^{\beta}}{\nu_{\phi_{f}}(v)^{(p-1) / p}} \mathrm{~d} x \mathrm{~d} v \mathrm{~d} s \\
\lesssim & o(1) \int_{0}^{t} \iint_{\Omega \times \mathbb{R}^{3}}\left|\nu_{\phi_{f}, w_{\tilde{\vartheta}}}^{1 / p} w_{\tilde{\vartheta}} \alpha_{f, \varepsilon}^{\beta} \partial f\right|^{p}+\left(1+\delta_{1} / \Lambda_{1}\right) \int_{0}^{t} \iint_{\Omega \times \mathbb{R}^{3}}\left|w_{\tilde{\vartheta}} f\right|^{p} .
\end{aligned}
$$

Here we have used the fact that, from (1.31) and (4.3)

$$
\begin{aligned}
& \alpha_{f, \varepsilon}(s, x, v) \\
\leq & \mathbf{1}_{s+1 \geq t_{\mathbf{b}}(s, x, v)}\left|v_{\mathbf{b}}(s, x, v)\right|+\mathbf{1}_{s \leq t_{\mathbf{b}}(s, x, v)+1} \\
\lesssim & 1+|v|+\int_{-1}^{0}\left|\nabla \phi_{f}(\tau, X(\tau ; s, x, v))\right| \mathrm{d} \tau \\
& +\int_{0}^{s}\left|\nabla \phi_{f}(\tau, X(\tau ; s, x, v))\right| \mathrm{d} \tau \\
\lesssim & \left(1+\left\|w_{\vartheta} f_{0}\right\|_{\infty}+\delta_{1} / \Lambda_{1}\right)\langle v\rangle,
\end{aligned}
$$

and from (4.1), $\frac{\alpha_{f, \varepsilon}(s, x, v)^{\beta}}{\nu_{\phi_{f}}(v)^{(p-1) / p}} \lesssim \delta_{1} / \Lambda_{1} \times \frac{\langle v\rangle^{\beta}}{\langle v\rangle^{(p-1) / p}} \lesssim \delta_{1} / \Lambda_{1}$.

From (4.25), the contribution of $\left|\Gamma_{v, \text { loss }}(f, f)\right|$ of (4.43) in (4.44) $\mathcal{G}$ is bounded by

$$
\begin{aligned}
& \left\|w_{\vartheta} f\right\|_{\infty} \int_{0}^{t} \iint_{\Omega \times \mathbb{R}^{3}} p\left|\alpha_{f, \varepsilon}^{\beta} \partial f\right|^{p-1} \alpha_{f, \varepsilon}(v)^{\beta}\langle v\rangle w_{\vartheta}(v)^{-1}\|f(s, x, \cdot)\|_{L^{p}} \\
\lesssim & \left\|w_{\vartheta} f\right\|_{\infty}\left\{\int_{0}^{t} \iint_{\Omega \times \mathbb{R}^{3}}\left|\alpha_{f, \varepsilon}^{\beta} \partial f\right|^{p}+\int_{0}^{t} \iint_{\Omega \times \mathbb{R}^{3}}|f|^{p}\right\},
\end{aligned}
$$

where we have used, from (4.49), $\alpha_{f, \varepsilon}(v)^{\beta}\langle v\rangle w_{\vartheta}(v)^{-1} \lesssim w_{\vartheta}(v)^{-1 / 2}$.

From (4.18) and (4.26), the contribution of $\left|\Gamma_{v \text {,gain }}\right|$ and $\left|K_{v} f\right|$ in (4.44) $\mathcal{G}$ is bounded by

$$
\begin{aligned}
& \left(1+\sup _{0 \leq s \leq t}\left\|w_{\vartheta} f\right\|_{\infty}\right) \\
& \times \int_{0}^{t} \iint_{\Omega \times \mathbb{R}^{3}} \alpha_{f, \varepsilon}^{\beta p}\left|w_{\tilde{\vartheta}} \partial f(v)\right|^{p-1} \int_{\mathbb{R}^{3}} \mathbf{k}_{\varrho}(v, u) \frac{w_{\tilde{\vartheta}}(v)}{w_{\tilde{\vartheta}}(u)}|f(u)| \\
\lesssim & o(1) \int_{0}^{t} \iint_{\Omega \times \mathbb{R}^{3}}\left|\nu_{\phi_{f}}^{1 / p} \alpha_{f, \varepsilon}^{\beta} \partial f\right|^{p} \\
& +\left(1+\sup _{0 \leq s \leq t}\left\|w_{\vartheta} f(s)\right\|_{\infty}\right) \int_{0}^{t} \iint_{\Omega \times \mathbb{R}^{3}}|f|^{p},
\end{aligned}
$$

where we have used, for $1 / p+1 / p^{*}=1$ and $0<\tilde{\varrho} \ll \varrho$, from (4.13), (4.14),

$$
\begin{aligned}
& \int_{\mathbb{R}^{3}} \alpha_{f, \varepsilon}(v)^{\beta p}\left|w_{\tilde{\vartheta}} \partial f(v)\right|^{p-1} \int_{\mathbb{R}^{3}} \mathbf{k}_{\tilde{\varrho}}(v, u) w_{\tilde{\vartheta}}(u)|f(u)| \mathrm{d} u \mathrm{~d} v \\
\lesssim & \int_{\mathbb{R}^{3}} \alpha_{f, \varepsilon}(v)^{\beta p}\left|w_{\tilde{\vartheta}} \partial f(v)\right|^{p-1} \int_{\mathbb{R}^{3}} \mathbf{k}_{\tilde{\varrho}}(v, u)^{1 / p^{*}} \mathbf{k}_{\tilde{\varrho}}(v, u)^{1 / p}\left|w_{\tilde{\vartheta}} f(u)\right| \mathrm{d} u \mathrm{~d} v \\
\lesssim & \int_{\mathbb{R}^{3}} \frac{\alpha_{f, \varepsilon}(v)^{\beta}}{\langle v\rangle^{\frac{p-1}{p}}}\left|\langle v\rangle^{1 / p} w_{\tilde{\vartheta}} \alpha_{f, \varepsilon}^{\beta} \partial f(v)\right|^{p-1} \\
& \times\left(\int_{\mathbb{R}^{3}} \mathbf{k}_{\tilde{\varrho}}(v, u) \mathrm{d} u\right)^{1 / p^{*}}\left(\int_{\mathbb{R}^{3}} \mathbf{k}_{\tilde{\varrho}}(v, u)\left|w_{\tilde{\vartheta}} f(u)\right|^{p} \mathrm{~d} u\right)^{1 / p} \mathrm{~d} v \\
\lesssim & \left(\int_{\mathbb{R}^{3}}\left|\langle v\rangle^{1 / p} w_{\tilde{\vartheta}} \alpha_{f, \varepsilon}^{\beta} \partial f(v)\right|^{p} \mathrm{~d} v\right)^{\frac{p-1}{p}}\left(\int_{\mathbb{R}^{3}} \int_{\mathbb{R}^{3}} \mathbf{k}_{\tilde{\varrho}}(v, u)\left|w_{\tilde{\vartheta}} f(u)\right|^{p} \mathrm{~d} u \mathrm{~d} v\right)^{\frac{1}{p}} \\
\lesssim & \left(\int_{\mathbb{R}^{3}}\left|\nu_{\phi_{f}}^{1 / p} w_{\tilde{\vartheta}} \alpha_{f, \varepsilon}^{\beta} \partial f\right|^{p}\right)^{\frac{p-1}{p}}\left(\int_{\mathbb{R}^{3}}\left|w_{\tilde{\vartheta}} f\right|^{p}\right)^{\frac{1}{p}} \cdot
\end{aligned}
$$


Note that from the standard elliptic estimate and (1.16),

$$
\left\|\phi_{f}(t)\right\|_{W^{2, p}(\Omega)} \lesssim\left\|\int_{\mathbb{R}^{3}} f(t, x, v) \sqrt{\mu(v)} \mathrm{d} v\right\|_{L^{p}(\Omega)} \lesssim\|f(t)\|_{L^{p}\left(\Omega \times \mathbb{R}^{3}\right)} .
$$

Then from (4.52) we bound the contribution of $w_{\tilde{\vartheta}}(v)^{-1 / 2}\left(\left|\nabla \phi_{f}\right|+\left|\nabla^{2} \phi_{f}\right|\right)\left(1+\left\|w_{\vartheta} f\right\|_{\infty}\right)$ of (4.43) in (4.42) by

$$
\begin{aligned}
&\left(1+\left\|w_{\vartheta} f\right\|_{\infty}\right) \int_{0}^{t} \iint_{\Omega \times \mathbb{R}^{3}} p\left|w_{\tilde{\vartheta}} \alpha_{f, \varepsilon}^{\beta} \partial f\right|^{p-1} \frac{\alpha_{f, \varepsilon}(v)^{\beta}}{w_{\tilde{\vartheta}}(v)^{1 / 2}}\left(\left|\nabla \phi_{f}\right|+\left|\nabla^{2} \phi_{f}\right|\right) \\
& \lesssim\left(1+\left\|w_{\vartheta} f\right\|_{\infty}\right) \int_{0}^{t} \iint_{\Omega \times \mathbb{R}^{3}}\left|w_{\tilde{\vartheta}} \alpha_{f, \varepsilon}^{\beta} \partial f\right|^{p-1} w_{\tilde{\vartheta}}^{-1 / 4}\left(\left|\nabla \phi_{f}\right|+\left|\nabla^{2} \phi_{f}\right|\right) \\
& \lesssim\left(1+\left\|w_{\vartheta} f\right\|_{\infty}\right)\left\{\int_{0}^{t} \iint_{\Omega \times \mathbb{R}^{3}}\left|w_{\tilde{\vartheta}} \alpha_{f, \varepsilon}^{\beta} \partial f\right|^{p}\right. \\
&\left.+\int_{0}^{t} \int_{\Omega}\left\|\phi_{f}\right\|_{W^{2, p}}^{p} \int_{\mathbb{R}^{3}} w_{\tilde{\vartheta}}^{-p / 4}\right\} \\
& \lesssim\left(1+\left\|w_{\vartheta} f\right\|_{\infty}\right) \int_{0}^{t} \iint_{\Omega \times \mathbb{R}^{3}}\left|w_{\tilde{\vartheta}} \alpha_{f, \varepsilon}^{\beta} \partial f\right|^{p}+\int_{0}^{t} \iint_{\Omega \times \mathbb{R}^{3}}|f|^{p},
\end{aligned}
$$

where we have used, from (4.49), $\alpha_{f, \varepsilon}(v)^{\beta} w_{\vartheta}(v)^{-1 / 2} \lesssim w_{\vartheta}(v)^{-1 / 4}$.

Step 3. We focus on (4.46). With $N>0$, we split the $u$-integration of (4.46) into the integrations over $\{|u| \leq N\}$ and $\{|u| \geq N\}$.

For $\{|u| \geq N\}$ and $0<\tilde{\varrho} \ll \varrho$, by Holder inequality with $\frac{1}{p}+\frac{1}{p^{*}}=1$

$$
\begin{aligned}
& \int_{|u| \geq N} \alpha_{f, \varepsilon}^{\beta}(v) \mathbf{k}_{\tilde{\varrho}}(v, u)|\partial f(u)| \\
\leq & \alpha_{f, \varepsilon}^{\beta}(v)\left(\int_{|u| \geq N} \mathbf{k}_{\tilde{\varrho}}(v, u) \frac{1}{\alpha_{f, \varepsilon}(u)^{\beta p^{*}}}\right)^{1 / p^{*}} \\
& \times\left(\int_{|u| \geq N} \mathbf{k}_{\tilde{\varrho}}(v, u)\left|\alpha_{f, \varepsilon}^{\beta} \partial f(u)\right|^{p}\right)^{1 / p} \\
\lesssim & \alpha_{f, \varepsilon}^{\beta}(v)\left(\int_{|u| \geq N} \mathbf{k}_{\tilde{\varrho}}(v, u)\left|\alpha_{f, \varepsilon}^{\beta} \partial f(u)\right|^{p} \mathrm{~d} u\right)^{1 / p},
\end{aligned}
$$

where we have used Proposition 3 with $\beta q<\frac{p-1}{p} \frac{p}{p-1}=1$ from 4.1).

Then the contribution of $\{|u| \geq N\}$ in (4.46) is bounded by

$$
\begin{gathered}
\int_{0}^{t} \int_{\Omega} \int_{v \in \mathbb{R}^{3}}\left|\nu_{\phi_{f}}^{1 / p} w_{\tilde{\vartheta}} \alpha_{f, \varepsilon}^{\beta} \partial f(v)\right|^{p-1} \frac{\alpha_{f, \varepsilon}(v)^{\beta}}{\nu_{\phi_{f}}(v)^{\frac{p-1}{p}}} \\
\quad \times \int_{|u| \geq N} \mathbf{k}_{\varrho}(v, u) \frac{w_{\tilde{\vartheta}}(v)}{w_{\tilde{\vartheta}}(u)}\left|w_{\tilde{\vartheta}} \partial f(u)\right| \mathrm{d} u \mathrm{~d} v \mathrm{~d} x \mathrm{~d} s \\
\leq \int_{0}^{t} \int_{\Omega}\left(\int_{v}\left|\nu_{\phi_{f}}^{1 / p} w_{\tilde{\vartheta}} \alpha_{f, \varepsilon}^{\beta} \partial f(v)\right|^{p}\right)^{1 / q} \\
\times\left(\int_{|u| \geq N}\left|w_{\tilde{\vartheta}} \alpha_{f, \varepsilon}^{\beta} \partial f(u)\right|^{p} \int_{v} \mathbf{k}_{\tilde{\varrho}}(v, u)\right)^{1 / p} \\
\lesssim o(1) \int_{0}^{t}\left\|\nu_{\phi_{f}}^{1 / p} w_{\tilde{\vartheta}} \alpha_{f, \varepsilon}^{\beta} \partial f(s)\right\|_{p}^{p} \mathrm{~d} s+\int_{0}^{t}\left\|w_{\tilde{\vartheta}} \alpha_{f, \varepsilon}^{\beta} \partial f(s)\right\|_{p}^{p} \mathrm{~d} s
\end{gathered}
$$

where we have used, from (4.49), $\frac{\alpha_{f, \varepsilon}(v)^{\beta}}{\nu_{\phi}(v)^{\frac{p-1}{p}}} \lesssim 1$ for $\beta$ in (4.1), (4.13), and (4.14). 
The contribution of $\{|u| \leq N\}$ in (4.46) is bounded by, from the Hölder inequality,

$$
\begin{aligned}
& \int_{0}^{t} \int_{\Omega} \int_{\mathbb{R}^{3}}\left|\nu_{\phi_{f}}^{1 / p} w_{\tilde{\vartheta}} \alpha_{f, \varepsilon}^{\beta} \partial f(v)\right|^{p-1} \\
& \times \int_{|u| \leq N} \mathbf{k}_{\varrho}(v, u) \frac{w_{\tilde{\vartheta}}(v)}{w_{\tilde{\vartheta}}(u)} \frac{\alpha_{f, \varepsilon}(v)^{\beta}\left|w_{\tilde{\vartheta}} \alpha_{f, \varepsilon}^{\beta} \partial f(u)\right|}{\nu_{\phi_{f}}(v)^{(p-1) / p} \alpha_{f, \varepsilon}(u)^{\beta}} \mathrm{d} u \mathrm{~d} v \mathrm{~d} x \mathrm{~d} s \\
& \leq \int_{0}^{t}\left\|\nu_{\phi_{f}}^{1 / p} w_{\tilde{\vartheta}} \alpha_{f, \varepsilon}^{\beta} \partial f(s)\right\|_{p}^{p-1}
\end{aligned}
$$

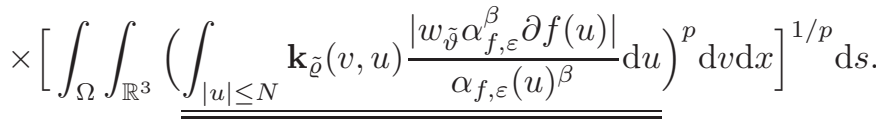

where we have used (4.13) and the fact $\alpha_{f, \varepsilon}^{\beta} / \nu_{\phi_{f}}^{\frac{p-1}{p}} \lesssim 1$ from (4.49) and (4.1).

By the Hölder inequality, we bound an underlined $u$-integration inside (4.56) as

$$
\left\|w_{\tilde{\vartheta}} \alpha_{f, \varepsilon}^{\beta} \partial f(\cdot)\right\|_{L^{p}\left(\mathbb{R}^{3}\right)} \times\left(\int_{\mathbb{R}^{3}} \frac{e^{-p^{*} \tilde{\varrho}|v-u|^{2}}}{|v-u|^{p^{*}}} \frac{\mathbf{1}_{|u| \leq N}}{\alpha_{f, \varepsilon}(u)^{\beta p^{*}}} \mathrm{~d} u\right)^{1 / q},
$$

where $1 / p+1 / p^{*}=1$.

It is important to note that

$$
\left(\int_{\mathbb{R}^{3}} \frac{e^{-p^{*} \tilde{\varrho}|v-u|^{2}}}{|v-u|^{p^{*}}} \frac{\mathbf{1}_{|u| \leq N}}{\alpha_{f, \varepsilon}(u)^{\beta p^{*}}} \mathrm{~d} u\right)^{1 / p^{*}} \leq\left|\frac{1}{|\cdot| p^{*}} * \frac{\mathbf{1}_{|\cdot| \leq N}}{\alpha_{f, \varepsilon}(\cdot)^{p^{*} \beta}}\right|^{1 / p^{*}} .
$$

By the Hardy-Littlewood-Sobolev inequality with

$$
1+\frac{1}{p / p^{*}}=\frac{1}{3 / p^{*}}+\frac{1}{\frac{3}{2} \frac{p-1}{p}}
$$

we have

$$
\begin{aligned}
& \left\|\left|\frac{1}{|\cdot| p^{*}} * \frac{\mathbf{1}_{|\cdot| \leq N}}{\alpha_{f, \varepsilon}(\cdot)^{p^{*} \beta}}\right|^{1 / p^{*}}\right\| \\
& =\left\|\frac{1}{|\cdot| p^{*}} * \frac{\mathbf{1}_{|\cdot| \leq N}}{\alpha_{f, \varepsilon}(\cdot)^{p^{*} \beta}}\right\|_{L^{p / p^{*}}\left(\mathbb{R}^{3}\right)}^{1 / p^{*}} \\
& \lesssim\left\|\frac{\mathbf{1}_{|\cdot| \leq N}}{\alpha_{f, \varepsilon}(\cdot)^{p^{*} \beta}}\right\|_{L^{\frac{3(p-1)}{2 p}}}^{1 / p^{*}}\left(\mathbb{R}^{3}\right) \\
& \lesssim\left(\int_{\mathbb{R}^{3}} \frac{\mathbf{1}_{|v| \leq N}}{\alpha_{f, \varepsilon}(v)^{\frac{p}{p-1} \beta \frac{3(p-1)}{2 p}}} \mathrm{~d} v\right)^{\frac{2 p}{3(p-1)} \frac{p-1}{p}} \\
& =\left(\int_{\mathbb{R}^{3}} \frac{\mathbf{1}_{|v| \leq N}}{\alpha_{f, \varepsilon}(v)^{3 \beta / 2}} \mathrm{~d} v\right)^{2 / 3} .
\end{aligned}
$$

For $3<p<6$, we have $\frac{3}{2} \frac{p-2}{p}<1$ and $\frac{2}{3}<\frac{p-1}{p}$. Importantly from (4.1) we have $\frac{3 \beta}{2}<1$. Now we apply (3.2) in Proposition 3 to conclude that

$$
\left(\int_{\mathbb{R}^{3}} \frac{\mathbf{1}_{|v| \leq M}}{\alpha_{f, \varepsilon}(v)^{3 \beta / 2}} \mathrm{~d} v\right)^{2 / 3} \lesssim_{p, \beta, M, \Omega} 1 .
$$

Finally from (4.56), (4.57), (4.58), (4.59), and (4.55) we bound

$$
\begin{aligned}
\text { (4.46) } \lesssim & o(1) \int_{0}^{t}\left\|\nu_{\phi}^{1 / p} w_{\tilde{\vartheta}} \alpha_{f, \varepsilon}^{\beta} \partial f\right\|_{p}^{p} \\
& +\left(1+\sup _{0 \leq s \leq t}\left\|w_{\vartheta} f(s)\right\|_{\infty}\right) \int_{0}^{t}\left\|w_{\tilde{\vartheta}} \alpha_{f, \varepsilon}^{\beta} \partial f\right\|_{p}^{p} .
\end{aligned}
$$


Collecting terms from (4.45), (4.46), (4.47), (4.48), (4.50), (4.51), (4.53), (4.55), and (4.60)

$$
\begin{aligned}
(14.44) \mathcal{G} \lesssim & \left(o(1)+\sup _{0 \leq s \leq t}\left\|w_{\vartheta} f(s)\right\|_{\infty}\right) \int_{0}^{t}\left\|\nu_{\phi_{f}}^{1 / p} w_{\tilde{\vartheta}} \alpha_{f, \varepsilon}^{\beta} \partial f\right\|_{p}^{p} \\
+ & \left(1+\sup _{0 \leq s \leq t}\left\|w_{\vartheta} f(s)\right\|_{\infty}+\sup _{0 \leq s \leq t}\left\|\nabla^{2} \phi_{f}(s)\right\|_{\infty}\right) \\
& \times \int_{0}^{t}\left\|w_{\tilde{\vartheta}} \alpha_{f, \varepsilon}^{\beta} \partial f\right\|_{p}^{p} \\
& +\left(1+\sup _{0 \leq s \leq t}\left\|w_{\vartheta} f(s)\right\|_{\infty}+\delta_{1} / \Lambda_{1}\right) \int_{0}^{t}\left\|w_{\tilde{\vartheta}} f\right\|_{p}^{p} .
\end{aligned}
$$

Step 4. We focus on (4.44) $\gamma_{-}$. From (4.27) and (4.28),

$$
\begin{aligned}
& \int_{n(x) \cdot v<0}|n(x) \cdot v|^{\beta p}\left|w_{\tilde{\vartheta}} \nabla_{x, v} f(t, x, v)\right|^{p}|n(x) \cdot v| \mathrm{d} v \\
& \lesssim \int_{n(x) \cdot v<0}\langle v\rangle^{p} \mu(v)^{\frac{p}{2}} w_{\tilde{\vartheta}}^{p} \\
& \quad \times\left.\left(|n(x) \cdot v|^{\beta p+1}+|n(x) \cdot v|^{(\beta-1) p+1}\right)|[4.28)|\right|^{p} \mathrm{~d} v .
\end{aligned}
$$

Note that for $0<\tilde{\vartheta} \ll_{p} 1$ we have $\mu(v)^{\frac{p}{2}} w_{\tilde{\vartheta}}^{p} \lesssim e^{C|v|^{2}}$ for some $C>0$ when $|v| \gg 1$.

On the other hand, from (4.1), we have

$$
(\beta-1) p+1>\frac{p-2}{p} p-p+1=-1, \quad|n(x) \cdot v|^{(\beta-1) p+1} \in L_{l o c}^{1}\left(\mathbb{R}^{3}\right) .
$$

Now we bound $|(4.28)|^{p}$. For the first line of (4.28), we split the $u$-integration into $\gamma_{+}^{\varepsilon}(x) \cup \gamma_{+}(x) \backslash \gamma_{+}^{\varepsilon}(x)$ where $\varepsilon$ is small but satisfies (2.51). By the Hölder inequality

$$
\begin{aligned}
& \left\{\int_{n(x) \cdot u>0}\left|w_{\tilde{\vartheta}} \alpha_{f, \varepsilon}^{\beta} \nabla_{x, v} f(s, x, u)\right|\left\{w_{\tilde{\vartheta}} \alpha_{f, \varepsilon}(u)\right\}^{-\beta}\right. \\
& \times\langle u\rangle \sqrt{\mu(u)}\{n(x) \cdot u\} \mathrm{d} u\}^{p} \\
& \lesssim\left\{\int_{\gamma_{+}^{\varepsilon}(x)}\left|w_{\tilde{\vartheta}} \alpha_{f, \varepsilon}^{\beta} \nabla_{x, v} f(s, x, u)\right|^{p}\{n(x) \cdot u\} \mathrm{d} u\right\} \\
& \quad \times\left\{\int_{\gamma_{+}^{\varepsilon}(x)}\left\{w_{\tilde{\vartheta}} \alpha_{f, \varepsilon}(u)\right\}^{-\beta p^{*}}|n(x) \cdot u| \mu^{\frac{q}{4}} \mathrm{~d} u\right\}^{p / p^{*}} \\
& +\left\{\int_{\gamma_{+}(x) \backslash \gamma_{+}^{\varepsilon}(x)}\left|w_{\tilde{\vartheta}} \alpha_{f, \varepsilon}^{\beta} \nabla_{x, v} f(s, x, u)\right|^{p} \mu^{\frac{p}{8}}\{n(x) \cdot u\} \mathrm{d} u\right\} \\
& \quad \times\left\{\int_{\gamma_{+}(x) \backslash \gamma_{+}^{\varepsilon}(x)}\left\{w_{\tilde{\vartheta}} \alpha_{f, \varepsilon}(s, x, u)\right\}^{-\beta p^{*}}|n(x) \cdot u| \mu^{\frac{p^{*}}{8}} \mathrm{~d} u\right\}^{p / p^{*}},
\end{aligned}
$$

for $p^{*}:=\frac{p}{p-1}$. Note that $\alpha_{f, \varepsilon}(s, x, u) \neq|n(x) \cdot u|$ for $(x, u) \in \gamma_{+}$in general. From (4.1), $\beta p^{*}<1$. From (3.2) and (3.3) with $v=0$, we have $\alpha_{f, \varepsilon}^{-\beta p^{*}}|n(x) \cdot u| \lesssim \alpha_{f, \varepsilon}^{-\beta p^{*}} \in L_{l o c}^{1}\left(\left\{u \in \mathbb{R}^{3}\right\}\right)$. Since $\mathbf{1}_{\gamma_{+}^{\varepsilon}(x)}(v) \downarrow 0$ almost everywhere in $\mathbb{R}^{3}$ as $\varepsilon \downarrow 0$, by the dominant convergence theorem, for (4.4), we choose $\varepsilon:=\frac{2 \delta_{1}}{\Lambda_{1}} \ll_{\Omega} 1$

$$
\begin{aligned}
(4.64) \lesssim & o(1) \int_{\gamma_{+}^{\varepsilon}(x)}\left|w_{\tilde{\vartheta}} \alpha_{f, \varepsilon}^{\beta} \nabla_{x, v} f(s, x, u)\right|^{p}\{n(x) \cdot u\} \mathrm{d} u \\
& +\int_{\gamma_{+}(x) \backslash \gamma_{+}^{\varepsilon}(x)}\left|w_{\tilde{\vartheta}} \alpha_{f, \varepsilon}^{\beta} \nabla_{x, v} f(s, x, u)\right|^{p} \mu(u)^{p / 8}\{n(x) \cdot u\} \mathrm{d} u .
\end{aligned}
$$


From Lemma 7 and (4.42), the last term of (4.65) has a bound as

$$
\begin{aligned}
& \int_{0}^{t} \int_{\partial \Omega} \int_{\gamma_{+}(x) \backslash \gamma_{+}^{\varepsilon}(x)}\left|w_{\tilde{\vartheta}} \alpha_{f, \varepsilon}^{\beta} \nabla_{x, v} f(s, x, u)\right|^{p} \mu(u)^{p / 8}\{n(x) \cdot u\} \mathrm{d} u \mathrm{~d} S_{x} \mathrm{~d} s \\
& \lesssim\left\|w_{\tilde{\vartheta}} \alpha_{f, \varepsilon}^{\beta} \nabla_{x, v} f(0) \mu^{1 / 8}\right\|_{p}^{p}+\int_{0}^{t}\left\|w_{\tilde{\vartheta}} \alpha_{f, \varepsilon}^{\beta} \nabla_{x, v} f\right\|_{p}^{p}+\text { (4.67), }
\end{aligned}
$$

where, from 4.39), 4.40,

$$
\begin{aligned}
& \int_{0}^{t} \iint_{\Omega \times \mathbb{R}^{3}}\left|\partial_{t}+v \cdot \nabla_{x}-\nabla_{x} \phi_{f} \cdot \nabla_{v}+\nu_{\phi_{f}, w_{\tilde{\vartheta}}}\right| \\
\times & \times w_{\tilde{\vartheta}} \mu^{1 / 8} \alpha_{f, \varepsilon}^{\beta}\left|\nabla_{x, v} f\right|^{p} \\
\leq & \int_{0}^{t} \iint_{\Omega \times \mathbb{R}^{3}} p \alpha_{f, \varepsilon}^{\beta p}\left|\nabla_{x, v} f\right|^{p-1}\left|w_{\tilde{\vartheta}} \mu^{1 / 8}\right|^{p}|\mathcal{G}| \\
\quad+ & \int_{0}^{t} \iint_{\Omega \times \mathbb{R}^{3}}\left|\nabla_{x} \phi_{f}\right| \mu^{0+}\left|\alpha_{f, \varepsilon}^{\beta} \nabla_{x, v} f\right|^{p} .
\end{aligned}
$$

Clearly (4.68) $\lesssim 4.61$. And, from (4.3),

$$
\text { (4.69) } \lesssim \delta_{1} \int_{0}^{t}\left\|w_{\tilde{\vartheta}} \alpha_{f, \varepsilon}^{\beta} \nabla_{x, v} f\right\|_{p}^{p}
$$

Now we consider the third term of (4.28). From the trace theorem $W^{1, p}(\Omega) \rightarrow W^{1-\frac{1}{p}, p}(\partial \Omega)$ and (4.52)

$$
\left\|\nabla \phi_{f}\right\|_{L^{p}(\partial \Omega)} \lesssim\left\|\nabla \phi_{f}\right\|_{W^{1-\frac{1}{p}, p}(\partial \Omega)} \lesssim\left\|\nabla \phi_{f}\right\|_{W^{1, p}(\Omega)} \lesssim\left\|w_{\tilde{\vartheta}} f\right\|_{L^{p}\left(\Omega \times \mathbb{R}^{3}\right)}
$$

Then

$$
\begin{aligned}
& \left\{\int_{\partial \Omega} \int_{n(x) \cdot u>0}\left(\langle u\rangle\left\|w_{\vartheta} f\right\|_{\infty}+\mu(u)^{\frac{1}{4}}\right)\left|\nabla \phi_{f}\right| \sqrt{\mu(u)}\{n(x) \cdot u\} \mathrm{d} u \mathrm{~d} S_{x}\right\}^{p} \\
& \lesssim\left(1+\left\|w_{\vartheta} f\right\|_{\infty}\right)\left\|\nabla \phi_{f}\right\|_{L^{p}(\partial \Omega)}^{p} \\
& \lesssim\left(1+\left\|w_{\vartheta} f\right\|_{\infty}\right)\left\|w_{\tilde{\vartheta}} f\right\|_{L^{p}\left(\Omega \times \mathbb{R}^{3}\right)}^{p} .
\end{aligned}
$$

For the second term of (4.28), by the Hölder inequality with $\frac{1}{p}+\frac{1}{q}=1$ for $3<p<6$

$$
\begin{aligned}
& \left\{\int_{n \cdot u>0}\langle u\rangle|f|+\left(1+\left\|w_{\vartheta} f\right\|_{\infty}\right) \int_{\mathbb{R}^{3}} \frac{\mathbf{k}_{\varrho}\left(u, u^{\prime}\right)^{1 / q}}{\left|n \cdot u^{\prime}\right|^{1 / p}} \mathbf{k}_{\varrho}\left(u, u^{\prime}\right)^{1 / p}\left|f\left(u^{\prime}\right)\right|\right. \\
& \left.\times\left|n \cdot u^{\prime}\right|^{1 / p} \mathrm{~d} u^{\prime} \sqrt{\mu}\{n \cdot u\} \mathrm{d} u\right\}^{p} \\
& \lesssim \int_{n \cdot u>0}|f|^{p}\{n \cdot u\} \mathrm{d} u \\
& +\left(1+\left\|w_{\vartheta} f\right\|_{\infty}\right)\left(\int_{\mathbb{R}^{3}} \mathbf{k}_{\varrho}\left(u, u^{\prime}\right)\left|n \cdot u^{\prime}\right|^{-q / p} \mathrm{~d} u^{\prime}\right)^{p / q} \\
& \times \int_{\mathbb{R}^{3}} \int_{\mathbb{R}^{3}} \mathbf{k}_{\varrho}\left(u, u^{\prime}\right)\left|f\left(u^{\prime}\right)\right|^{p}\left|n \cdot u^{\prime}\right| \mathrm{d} u^{\prime} \mathrm{d} u \\
& \lesssim\left(1+\left\|w_{\vartheta} f\right\|_{\infty}\right) \int_{n \cdot u>0}|f|^{p}\{n \cdot u\} \mathrm{d} u . \\
& 39
\end{aligned}
$$


Collecting terms from (4.62), (4.65), (4.67), (4.71), and (4.72) we derive that

$$
\begin{aligned}
& (4.44) \gamma_{-} \\
\lesssim & \left\|w_{\tilde{\vartheta}} \alpha_{f, \varepsilon}^{\beta} \nabla_{x, v} f(0) \mu(u)^{1 / 8}\right\|_{p}^{p} \\
& +o(1) \int_{0}^{t}\left|w_{\tilde{\vartheta}} \alpha_{f, \varepsilon}^{\beta} \partial f\right|_{p,+}^{p} \\
& +\left(o(1)+\sup _{0 \leq s \leq t}\left\|w_{\vartheta} f(s)\right\|_{\infty}\right) \int_{0}^{t}\left\|\nu_{\phi_{f}}^{1 / p} w_{\tilde{\vartheta}} \alpha_{f, \varepsilon}^{\beta} \partial f\right\|_{p}^{p} \\
& +\left(1+\sup _{0 \leq s \leq t}\left\|w_{\vartheta} f(s)\right\|_{\infty}+\sup _{0 \leq s \leq t}\left\|\nabla^{2} \phi_{f}(s)\right\|_{\infty}\right) \int_{0}^{t}\left\|\alpha_{f, \varepsilon}^{\beta} w_{\tilde{\vartheta}} \partial f\right\|_{p}^{p} \\
& +\left(1+\sup _{0 \leq s \leq t}\left\|w_{\vartheta} f(s)\right\|_{\infty}\right) \int_{0}^{t}\left(\left\|w_{\tilde{\vartheta}} f\right\|_{p}^{p}+\left|w_{\tilde{\vartheta}} f\right|_{p,+}^{p}\right) .
\end{aligned}
$$

Step 5. From (4.44), 4.61), 4.73) we have

$$
\begin{gathered}
\left\|w_{\tilde{\vartheta}} \alpha_{f, \varepsilon}^{\beta} \partial f(t)\right\|_{p}^{p}+\int_{0}^{t}\left\|\nu_{\phi_{f}, w_{\vartheta}}^{1 / p} w_{\tilde{\vartheta}} \alpha_{f, \varepsilon}^{\beta} \partial f(t)\right\|_{p,+}^{p}+\int_{0}^{t}\left|w_{\tilde{\vartheta}} \alpha_{f, \varepsilon}^{\beta} \partial f\right|_{p}^{p} \\
\lesssim\left\|w_{\tilde{\vartheta}} \alpha_{f, \varepsilon}^{\beta} \partial f(0)\right\|_{p}^{p}+\left(1+\sup _{0 \leq s \leq t}\|w f(s)\|_{\infty}\right) \int_{0}^{t}\left(\left\|w_{\tilde{\vartheta}} f\right\|_{p}^{p}+\left|w_{\tilde{\vartheta}} f\right|_{p,+}^{p}\right) \\
+\left(1+\sup _{0 \leq s \leq t}\|w f(s)\|_{\infty}+\sup _{0 \leq s \leq t}\left\|\nabla^{2} \phi_{f}(s)\right\|_{\infty}\right) \int_{0}^{t}\left\|w_{\tilde{\vartheta}} \alpha_{f, \varepsilon}^{\beta} \partial f(t)\right\|_{p}^{p} .
\end{gathered}
$$

Multiplying small number to (4.74) and adding to (4.38) we derive that

$$
\begin{aligned}
& \left\|w_{\tilde{\vartheta}} f(t)\right\|_{p}^{p}+\left\|w_{\tilde{\vartheta}} \alpha_{f, \varepsilon}^{\beta} \partial f(t)\right\|_{p}^{p} \\
& +\int_{0}^{t}\left(\left\|\nu_{\phi_{f}, w_{\tilde{\vartheta}}}^{1 / p} w_{\tilde{\vartheta}} f\right\|_{p}^{p}+\left\|\nu_{\phi_{f}, w_{\tilde{\vartheta}}}^{1 / p} w_{\tilde{\vartheta}} \alpha_{f, \varepsilon}^{\beta} \partial f\right\|_{p}^{p}\right) \\
& +\int_{0}^{t}\left|w_{\tilde{\vartheta}} f\right|_{p,+}^{p}+\int_{0}^{t}\left|w_{\tilde{\vartheta}} \alpha_{f, \varepsilon}^{\beta} \partial f\right|_{p,+}^{p} \\
& \lesssim\left\|w_{\tilde{\vartheta}} f(0)\right\|_{p}^{p}+\left\|w_{\tilde{\vartheta}} \alpha_{f, \varepsilon}^{\beta} \partial f(0)\right\|_{p}^{p}+\int_{0}^{t}\left\|w_{\tilde{\vartheta}} f\right\|_{p}^{p} \\
& +\left(1+\sup _{0 \leq s \leq t}\left\|\nabla^{2} \phi_{f}(s)\right\|_{\infty}\right) \int_{0}^{t}\left\|w_{\tilde{\vartheta}} \alpha_{f, \varepsilon}^{\beta} \partial f\right\|_{p}^{p},
\end{aligned}
$$

where we have used (4.2). Then by the Gronwall's inequality, we deduce (4.5).

\section{5. $L_{x}^{3} L_{v}^{1+}$-Estimate of $\nabla_{v} f$ AND $L^{1+}$-Stability}

Proposition 5. Assume $f$ and $\phi_{f}$ solve (1.15), (1.16), (1.17), and satisfy estimates (1.36), (1.37), 4.3) with the condition (4.4). We also assume extra initial condition

$$
\left\|w_{\tilde{\vartheta}} \nabla_{v} f_{0}\right\|_{L_{x, v}^{3}}<\infty .
$$

Then

$$
\left\|\nabla_{v} f(t)\right\|_{L_{x}^{3}(\Omega) L_{v}^{1+\delta}\left(\mathbb{R}^{3}\right)} \lesssim t 1 \text { for all } t \geq 0 .
$$

Once we have Proposition 5 , we can prove the following stability result.

Proposition 6. Suppose $f$ and $g$ solve (1.15), (1.16), (1.17), and satisfy (1.36). Also we assume $f, g$ satisfy (5.2). Then

$$
\begin{aligned}
& \|f(t)-g(t)\|_{L^{1+\delta}\left(\Omega \times \mathbb{R}^{3}\right)}^{1+\delta}+\int_{0}^{t}\|f(s)-g(s)\|_{L^{1+\delta}(\gamma)}^{1+\delta} \mathrm{d} s \\
& \lesssim_{t}\left\|f_{0}-g_{0}\right\|_{L^{1+\delta}\left(\Omega \times \mathbb{R}^{3}\right)}^{1+\delta}{ }_{40}
\end{aligned}
$$


Proof. Assume that $f$ and $g$ solve (4.39). Then

$$
\begin{aligned}
& \partial_{t}[f-g]+v \cdot \nabla_{x}[f-g]-\nabla_{x} \phi_{f} \cdot \nabla_{v}[f-g] \\
& +\frac{v}{2} \cdot \nabla_{x} \phi_{f}[f-g]+\nu[f-g] \\
= & \nabla_{x} \phi_{f-g} \cdot \nabla_{v} g \\
& +K[f-g]-\frac{v}{2} \cdot \nabla_{x} \phi_{f-g} g+\Gamma(f, f)-\Gamma(g, g)-v \cdot \nabla_{x} \phi_{f-g} \sqrt{\mu} .
\end{aligned}
$$

By Lemma 6 for $L^{1+\delta}$-space with $0<\delta \ll 1$, we obtain

$$
\begin{aligned}
& \|[f-g](t)\|_{1+\delta}^{1+\delta}+\int_{0}^{t}\left\|\nu_{\phi_{f}}^{1 / 1+\delta}[f-g]\right\|_{1+\delta}^{1+\delta}+\int_{0}^{t}|[f-g]|_{1+\delta,+}^{1+\delta} \\
\leq & \|[f-g](0)\|_{1+\delta}^{1+\delta}+\int_{0}^{t} \iint_{\Omega \times \mathbb{R}^{3}} \mid \text { RHS of (5.4) } \| f-\left.g\right|^{\delta} \\
& +\int_{0}^{t}|[f-g]|_{1+\delta,-}^{1+\delta} .
\end{aligned}
$$

Step 1. For $0<\delta \ll 1$, by the Hölder inequality with $1=\frac{1}{\frac{3(1+\delta)}{2-\delta}}+\frac{1}{3}+\frac{1}{\frac{1+\delta}{\delta}}$ and the Sobolev embedding $W^{1,1+\delta}(\Omega) \subset L^{\frac{3(1+\delta)}{2-\delta}}(\Omega)$ when $\Omega \subset \mathbb{R}^{3}$,

$$
\begin{aligned}
& \int_{0}^{t} \iint_{\Omega \times \mathbb{R}^{3}}\left|\nabla_{x} \phi_{f-g} \cdot \nabla_{v} g \| f-g\right|^{\delta} \\
& \lesssim \int_{0}^{t}\left\|\nabla_{x} \phi_{f-g}\right\|_{L_{x}^{\frac{3(1+\delta)}{2-\delta}}}\left\|\nabla_{v} g\right\|_{L_{x}^{3} L_{v}^{1+\delta}}\left\||f-g|^{\delta}\right\|_{L_{x, v}^{\frac{1+\delta}{\partial}}} \\
& \lesssim \sup _{0 \leq s \leq t}\left\|\nabla_{v} g(s)\right\|_{L_{x}^{3} L_{v}^{1+\delta}} \times \int_{0}^{t}\|[f-g](s)\|_{1+\delta}^{1+\delta} \mathrm{d} s .
\end{aligned}
$$

A simple modification of (4.35) and (4.36) as

$$
\begin{aligned}
& \int_{0}^{t} \int_{x} \int_{v} \int_{u} \mathbf{k}_{\varrho}(v, u)|f(u)-g(u)||f(v)-g(v)|^{\delta} \\
\lesssim & \int_{0}^{t} \int_{x} \int_{v} \int_{u} \mathbf{k}_{\varrho}(v, u)^{\frac{1}{1+\delta}}|f(u)-g(u)| \mathbf{k}_{\varrho}(v, u)^{\frac{\delta}{1+\delta}}|f(v)-g(v)|^{\delta} \\
\lesssim & \int_{0}^{t} \int_{x} \int_{v}|f(v)-g(v)|^{1+\delta} \int_{u} \mathbf{k}_{\varrho}(v, u) \\
\lesssim & \int_{0}^{t}\|f-g\|_{1+\delta}^{1+\delta}
\end{aligned}
$$

leads to

$$
\begin{aligned}
& \int_{0}^{t} \iint_{\Omega \times \mathbb{R}^{3}} \mid \text { the } 2^{\text {nd }} \text { line of RHS of (5.4) }|| f-\left.g\right|^{\delta} \\
\lesssim & \sup _{0 \leq s \leq t}\left\{1+\left\|w_{\vartheta} f(s)\right\|_{\infty}+\left\|w_{\vartheta} g(s)\right\|_{\infty}\right\} \int_{0}^{t}\|f-g\|_{1+\delta}^{1+\delta} .
\end{aligned}
$$

Then following the proof of (4.37) and applying (5.6) to (5.7), we can obtain

$$
\begin{aligned}
& \int_{0}^{t}|[f-g]|_{1+\delta,-}^{1+\delta} \\
& \lesssim o(1) \int_{0}^{t}|[f-g]|_{1+\delta,+}^{1+\delta}+\|[f-g](0)\|_{1+\delta}^{1+\delta} \\
& +\sup _{0 \leq s \leq t}\left\{1+\left\|\nabla_{v} g(s)\right\|_{L_{x}^{3} L_{v}^{1+\delta}}+\left\|w_{\vartheta} f(s)\right\|_{\infty}+\left\|w_{\vartheta} g(s)\right\|_{\infty}\right\} \\
& \quad \times \int_{0}^{t}\|f-g\|_{1+\delta}^{1+\delta} .
\end{aligned}
$$


Using (5.2), (5.5), (5.6), (5.7), (5.8) and applying the Gronwall inequality, we prove $L^{1+\delta}$-stability (5.3) for all time $t \geq 0$.

Proof of Proposition 5. Step 1. Note that from (1.15) and (4.31), we have

$$
\begin{aligned}
& {\left[\partial_{t}+v \cdot \nabla_{x}-\nabla_{x} \phi_{f} \cdot \nabla_{v}+\nu(v)+\frac{v}{2} \cdot \nabla_{x} \phi_{f}\right] \partial_{v} f } \\
= & -\partial_{x} f-\frac{1}{2} \partial_{x} \phi_{f} f-\partial_{v} \nu f+\partial_{v}(K f)+\partial_{v}(\Gamma(f, f))+\left|\nabla_{x} \phi_{f}\right|\langle v\rangle^{2} \sqrt{\mu},
\end{aligned}
$$

with the boundary bound for $(x, v) \in \gamma_{-}$

$$
\left|\partial_{v} f\right| \lesssim|v| \sqrt{\mu} \int_{n \cdot u>0}|f| \sqrt{\mu}\{n \cdot u\} \mathrm{d} u \text { on } \gamma_{-} .
$$

From (4.11), (4.18), (4.23), (4.24), (4.25), and (4.26), we obtain the following bound along the characteristics

$$
\begin{aligned}
& \left|\partial_{v} f(t, x, v)\right| \\
\leq & \mathbf{1}_{t_{\mathbf{b}}(t, x, v)>t}\left|\partial_{v} f(0, X(0 ; t, x, v), V(0 ; t, x, v))\right| \\
& +\mathbf{1}_{t_{\mathbf{b}}(t, x, v)<t} \mu\left(v_{\mathbf{b}}\right)^{\frac{1}{4}} \int_{n\left(x_{\mathbf{b}}\right) \cdot u>0}\left|f\left(t-t_{\mathbf{b}}, x_{\mathbf{b}}, u\right)\right| \sqrt{\mu}\left\{n\left(x_{\mathbf{b}}\right) \cdot u\right\} \mathrm{d} u \\
& +\int_{\max \left\{t-t_{\mathbf{b}}, 0\right\}}^{t}\left|\partial_{x} f(s, X(s ; t, x, v), V(s ; t, x, v))\right| \mathrm{d} s \\
& +\int_{\max \left\{t-t_{\mathbf{b}}, 0\right\}}^{t}\left(1+\left\|w_{\vartheta} f\right\|_{\infty}\right) \int_{\mathbb{R}^{3}} \mathbf{k}_{\varrho}(V(s), u)\left|\partial_{v} f(s, X(s), u)\right| \mathrm{d} u \mathrm{~d} s \\
& +\int_{\max \left\{t-t_{\mathbf{b}}, 0\right\}}^{t}\left|\nabla_{x} \phi_{f}(s, X(s ; t, x, v))\right| \mu^{1 / 4} \mathrm{~d} s \\
& +\int_{\max \left\{t-t_{\mathbf{b}}, 0\right\}}^{t}\left(1+\delta_{1}\right) \delta_{1}\left|w_{\tilde{\vartheta}}(V(s ; t, x, v))\right|^{-1} \mathrm{~d} s,
\end{aligned}
$$

where $\delta_{1}$ is in (4.3).

Note that if $|v|>2 \frac{\delta_{1}}{\Lambda_{1}}$, then from (4.3) and (4.4), for $0 \leq s \leq t$,

$$
\begin{aligned}
|V(s ; t, x, v)| & \geq|v|-\int_{0}^{t}\left|\nabla_{x} \phi(\tau ; t, x, v)\right| \mathrm{d} \tau \\
& \geq|v|-\delta_{1} / \Lambda_{1} \\
& \geq \frac{|v|}{2} .
\end{aligned}
$$

Therefore

We derive

$$
\sup _{s, t, x}\left\|\frac{1}{w_{\tilde{\vartheta}}(V(s ; t, x, v))}\right\|_{L_{v}^{r}} \lesssim 1 \text { for any } 1 \leq r \leq \infty .
$$

$$
\begin{aligned}
& \quad \|\left(\int _ { \Omega } \left(\int_{\mathbb{R}^{3}}\left|w_{\tilde{\vartheta}} \partial_{v} f(0, X(0), V(0))\right|_{L_{x}^{3} L_{v}^{1+\delta}}\right.\right. \\
& \left.\quad \times\left(\int_{\mathbb{R}^{3}} \frac{\mathrm{d} v}{\left|w_{\tilde{\vartheta}}(V(0))\right|^{(1+\delta) \frac{3}{2-\delta}}}\right)^{\frac{2-\delta}{1+\delta}}\right)^{1 / 3} \\
& \lesssim\left(\iint_{\Omega \times \mathbb{R}^{3}}\left|w_{\tilde{\vartheta}}(V(0)) \partial_{v} f(0, X(0), V(0))\right|^{3} \mathrm{~d} v \mathrm{~d} x\right)^{1 / 3} \\
& \lesssim\left\|w_{\tilde{\vartheta}} \partial_{v} f(0)\right\|_{L_{x, v}^{3}},
\end{aligned}
$$

where we have used a change of variables $(x, v) \mapsto(X(0 ; t, x, v), V(0 ; t, x, v))$ and (5.18).

Clearly

$$
\|(5.12)\|_{L_{x}^{3} L_{v}^{1+\delta}}+\|\left(\underline{5.16)}\left\|_{L_{x}^{3} L_{v}^{1+\delta}} \lesssim \sup _{0 \leq s \leq t}\right\| w_{\vartheta} f(s) \|_{\infty}\right.
$$


From $W^{1,2}(\Omega) \subset L^{6}(\Omega) \subset L^{2}(\Omega)$ for a bounded $\Omega \subset \mathbb{R}^{3}$, and the change of variables $(x, v) \mapsto(X(s ; t, x, v), V(s ; t, x, v))$ for fixed $s \in\left(\max \left\{t-t_{\mathbf{b}}, 0\right\}, t\right)$,

$$
\begin{aligned}
\|\left(\text { [5.15) } \|_{L_{x}^{3} L_{v}^{1+\delta}}\right. & \lesssim \int_{0}^{t}\left\|\mu^{1 / 8} \nabla_{x} \phi_{f}(s, X(s ; t, x, v))\right\|_{L_{x, v}^{3}}\left\|\mu^{1 / 8}\right\|_{L_{v}^{\frac{3(1+\delta)}{2-\delta}}} \\
& \lesssim \int_{0}^{t}\left\|\nabla_{x} \phi_{f}(s)\right\|_{L_{x}^{3}} \lesssim \int_{\max \left\{t-t_{\mathbf{b}}, 0\right\}}^{t}\left\|\phi_{f}(s)\right\|_{W_{x}^{2,2}} \\
& \lesssim \int_{0}^{t}\left\|w_{\tilde{\vartheta}} f(s)\right\|_{2} .
\end{aligned}
$$

Step 2. We claim

$$
\|\left(\underline{5.13)}\left\|_{L_{x}^{3} L_{v}^{1+\delta}} \lesssim \int_{0}^{t}\right\| w_{\tilde{\vartheta}} \alpha_{f, \varepsilon}^{\beta} \partial_{x} f(s) \|_{L_{x, v}^{p}}\right.
$$

Now we have for $3<p<6$, by the Hölder inequality $\frac{1}{1+\delta}=\frac{1}{\frac{p+p \delta}{p-1-\delta}}+\frac{1}{p}$,

$$
\begin{aligned}
& \|\| \int_{\max \left\{t-t_{\mathbf{b}}, 0\right\}}^{t} \partial_{x} f(s, X(s ; t, x, v), V(s ; t, x, v)) \mathrm{d} s\left\|_{L_{v}^{1+\delta}\left(\mathbb{R}^{3}\right)}\right\|_{L_{x}^{3}} \\
\lesssim & \|\| \int_{\max \left\{t-t_{\mathbf{b}}, 0\right\}}^{t} \frac{w_{\tilde{\vartheta}} \alpha_{f, \varepsilon}^{\beta} \partial_{x} f(s, X(s), V(s))}{w_{\tilde{\vartheta}} \alpha_{f, \varepsilon}(s, X(s), V(s))^{\beta}} \mathrm{d} s\left\|_{L_{v}^{1+\delta}\left(\mathbb{R}^{3}\right)}\right\|_{L_{x}^{3}} \\
\lesssim & \left\|\frac{w_{\tilde{\vartheta}}(v)^{-1}}{\alpha_{f, \varepsilon}(t, x, v)^{\beta}}\right\|_{L_{v}^{\frac{p+p \delta}{p-1-\delta}}\left(\mathbb{R}^{3}\right)} \\
& \times\|\| \int_{0}^{t} w_{\tilde{\vartheta}} \alpha_{f, \varepsilon}^{\beta} \partial_{x} f(s, X(s ; t, x, v), V(s ; t, x, v)) \mathrm{d} s\left\|_{L_{v}^{p}\left(\mathbb{R}^{3}\right)}\right\|_{L_{x}^{3}} \\
\lesssim & \left\|\frac{w_{\tilde{\vartheta}}(v)^{-1}}{\alpha_{f, \varepsilon}(t, x, v)^{\beta}}\right\|_{L_{v}^{\frac{p+p \delta}{p-1-\delta}}\left(\mathbb{R}^{3}\right)} \times \int_{0}^{t}\left\|w_{\tilde{\vartheta}} \alpha_{f, \varepsilon}^{\beta} \partial_{x} f(s)\right\|_{L_{x, v}^{p}} \mathrm{~d} s,
\end{aligned}
$$

where we have used $\alpha_{f, \varepsilon}(t, x, v)=\alpha_{f, \varepsilon}(s, X(s ; t, x, v), V(s ; t, x, v))$ for $t-t_{\mathbf{b}}(t, x, v) \leq s \leq t$ and the change of variables $(x, v) \mapsto(X(s ; t, x, v), V(s ; t, x, v))$ and the Minkowski inequality.

For $\beta$ in (1.34), we have $\beta \frac{p}{p-1}<1$ since $\frac{2}{3}<\frac{p-1}{p}$ for $3<p$. Therefore, we can choose $0<\delta \ll 1$ so that $\beta$ in (1.34) satisfies

$$
\beta \times \frac{p+p \delta}{p-1-\delta}<1
$$

We apply Proposition 3 to conclude that

$$
\sup _{t, x}\left\|\frac{w_{\tilde{\vartheta}}(v)^{-1}}{\alpha_{f, \varepsilon}(t, x, v)^{\beta}}\right\|_{L_{v}^{\frac{p+p \delta}{p-1-\delta}}}^{\frac{p+p \delta}{p-1-\delta}}=\sup _{t, x} \int_{\left.\mathbb{R}^{3}\right)} \frac{e^{-\tilde{\vartheta} \frac{p+p \delta}{p-1-\delta}|v|^{2}}}{\alpha_{f, \varepsilon}(t, x, v)^{\beta \frac{p+p \delta}{p-1-\delta}}} \mathrm{d} v \lesssim 1 .
$$

Finally, from (5.23), (5.25), and (1.37), we conclude the claim (5.22).

Step 3. We consider (5.14). We split the $u$-integration of (5.14) into two parts with $N \gg 1$ as

$$
\begin{aligned}
& \int_{|u| \leq N} \mathbf{k}_{\varrho}(V(s), u)\left|\nabla_{v} f(s, X(s), u)\right| \mathrm{d} u \\
+ & \int_{|u| \geq N} \mathbf{k}_{\varrho}(V(s), u)\left|\nabla_{v} f(s, X(s), u)\right| \mathrm{d} u .
\end{aligned}
$$


First we bound (5.26). From the change of variables $(x, v) \mapsto(X(s ; t, x, v), V(s ; t, x, v))$ for $t-t_{\mathbf{b}}(t, x, v) \leq$ $s \leq t$

$$
\begin{aligned}
& \left\|\int_{|u| \leq N} \mathbf{k}_{\varrho}(V(s ; t, x, v), u)\left|\nabla_{v} f(s, X(s ; t, x, v), u)\right| \mathrm{d} u\right\|_{L_{x}^{3} L_{v}^{3}} \\
= & \left\|\int_{|u| \leq N} \mathbf{k}_{\varrho}(v, u)\left|\nabla_{v} f(s, x, u)\right| \mathrm{d} u\right\|_{L_{x}^{3} L_{v}^{3}} .
\end{aligned}
$$

If $|v| \geq 2 N$ then $|v-u|^{2} \gtrsim|v|^{2}$ and $\mathbf{k}_{\varrho}(v, u) \lesssim \frac{e^{-C|v|^{2}}}{|v-u|^{2}}$ for $|v| \geq 2 N$ and $|u| \leq N$. For $0<\delta \ll 1$ with $\frac{3(1+\delta)}{1-2 \delta}>3$, 5.28

$$
\begin{aligned}
& \lesssim C_{N}\|\| \int_{|u| \leq N} \mathbf{k}_{\varrho}(v, u)\left|\nabla_{v} f(s, x, u)\right| \mathrm{d} u\left\|_{L_{v}^{\frac{3(1+\delta)}{1-2 \delta}}(\{|v| \leq 2 N\})}\right\|_{L_{x}^{3}} \\
& +\|\| e^{-C|v|^{2}}\left\|\left.\right|_{L_{v}^{3 / 2}}\right\| \int_{|u| \leq N} \frac{1}{|v-u|}\left|\nabla_{v} f(s, x, u)\right| \mathrm{d} u\left\|_{L_{v}^{\frac{3(1+\delta)}{1-2 \delta}}(\{|v| \geq 2 N\})}\right\|_{L_{x}^{3}} \\
& \lesssim\|\| \frac{1}{|v-\cdot|} *\left|\nabla_{v} f(s, x, \cdot)\right|\left\|_{L_{v}^{\frac{3(1+\delta)}{1-2 \delta}}}\right\|_{L_{x}^{3}} .
\end{aligned}
$$

Then by the Hardy-Littlewood-Sobolev inequality with $1+\frac{1}{\frac{3(1+\delta)}{1-2 \delta}}=\frac{1}{3}+\frac{1}{1+\delta}$, we derive that

$$
\left(\underline{5.29)} \lesssim\|\| \nabla_{v} f(s, x, v)\left\|_{L_{v}^{1+\delta}}\right\|_{L_{x}^{3}}=\left\|\nabla_{v} f(s)\right\|_{L_{x}^{3} L_{v}^{1+\delta}}\right.
$$

Combining the last estimate with (5.28), (5.29), we prove that

$$
\|\left(\underline{5.26)}\left\|_{L_{x}^{3} L_{v}^{1+\delta}} \lesssim\right\| \nabla_{v} f(s) \|_{L_{x}^{3} L_{v}^{1+\delta}} .\right.
$$

Now we consider (5.27). Choose $0<\iota \ll 1$. We write as

$$
\begin{aligned}
& =\int_{|u| \geq N} \frac{1}{w_{\tilde{\vartheta}}(V(s ; t, x, v))^{1-\iota}} \frac{w_{\tilde{\vartheta}}(V(s ; t, x, v))}{w_{\tilde{\vartheta}}(u)} \frac{\mathbf{k}_{\varrho}(V(s ; t, x, v), u)}{\alpha_{f, \varepsilon}(s, X(s ; t, x, v), u)^{\beta}} \\
& \quad \times \frac{w_{\tilde{\vartheta}}(u)}{w_{\tilde{\vartheta}}(V(s ; t, x, v))^{\iota}} \alpha_{f, \varepsilon}(s, X(s ; t, x, v), u)^{\beta}\left|\nabla_{v} f(s, X(s ; t, x, v), u)\right| \mathrm{d} u .
\end{aligned}
$$

By the Hölder inequality with $\frac{1}{p}+\frac{1}{p^{*}}=1$ with $3<p<6$

$$
\begin{aligned}
& \text { | (5.27) | } \\
& \lesssim \frac{1}{w_{\tilde{\vartheta}}(V(s ; t, x, v))^{1-\iota}} \\
& \times\left\|\frac{w_{\tilde{\vartheta}}(V(s ; t, x, v))}{w_{\tilde{\vartheta}}(u)} \frac{\mathbf{k}_{\varrho}(V(s ; t, x, v), u)}{\alpha_{f, \varepsilon}(s, X(s ; t, x, v), u)^{\beta}}\right\|_{L^{p^{*}}(\{|u| \geq N\})} \\
& \times \| \frac{w_{\tilde{\vartheta}}(u)}{w_{\tilde{\vartheta}}(V(s ; t, x, v))^{\iota}} \alpha_{f, \varepsilon}(s, X(s ; t, x, v), u)^{\beta} \\
& \times\left|\nabla_{v} f(s, X(s ; t, x, v), u)\right| \|_{L_{u}^{p}\left(\mathbb{R}^{3}\right)} .
\end{aligned}
$$


Then by the Hölder inequality with $\frac{1}{1+\delta}=\frac{1}{p}+\frac{1}{\frac{(1+\delta) p}{p-(1+\delta)}}$,

$$
\begin{aligned}
& \| \text { (5.27) } \|_{L_{v}^{1+\delta}} \\
& \lesssim\left\|\frac{1}{w_{\tilde{\vartheta}}(V(s ; t, x, v))^{1-\iota}}\right\|_{L_{v}^{p-(1+\delta)}} \\
& \times \sup _{v}\left\|\frac{w_{\tilde{\vartheta}}(V(s ; t, x, v))}{w_{\tilde{\vartheta}}(u)} \frac{\mathbf{k}_{\varrho}(V(s ; t, x, v), u)}{\alpha_{f, \varepsilon}(s, X(s ; t, x, v), u)^{\beta}}\right\|_{L^{p^{*}}(\{|u| \geq N\})} \\
& \times\|\| \frac{w_{\tilde{\vartheta}}(u)}{w_{\tilde{\vartheta}}(V(s ; t, x, v))^{\iota}} \alpha_{f, \varepsilon}(s, X(s ; t, x, v), u)^{\beta} \mid \nabla_{v} f(s, X(s ; t, x, v), u)\|\|_{L_{u}^{p}} \|_{L_{v}^{p}} .
\end{aligned}
$$

Note that, from (4.13), $\mathbf{k}_{\varrho}(v, u) \frac{e^{\tilde{v}|v|^{2}}}{e^{\hat{v}|u|^{2}}} \lesssim \mathbf{k}_{\tilde{\varrho}}(v, u)$ for some $0<\tilde{\varrho}<\varrho$. Hence we derive, using (‥18)

$$
\begin{aligned}
& \|\| \underline{(5.27)}\left\|_{L_{v}^{1+\delta}}\right\|_{L_{x}^{3}} \\
& \lesssim \Omega \sup _{X, V}\left\|\frac{e^{-\frac{\tilde{s}}{10}|V-u|^{2}}}{|V-u|} \frac{1}{\alpha_{f, \varepsilon}(s, X, u)^{\beta}}\right\|_{L^{p^{*}(\{|u| \geq N\})}} \\
& \quad \times\left\|\frac{\tilde{w}(u)}{\tilde{w}(V(s ; t, x, v))^{\iota}} \alpha_{f, \varepsilon}(s, X(s ; t, x, v), u)^{\beta}\left|\nabla_{v} f(s, X(s ; t, x, v), u)\right|\right\|_{L_{u, v, x}^{p}} .
\end{aligned}
$$

Finally using (3.3) in Proposition 3 with $\frac{p-2}{p-1}<\beta p^{*}<1$ from (1.34) and applying the change of variables $(x, v) \mapsto(X(s ; t, x, v), V(s ; t, x, v))$, we derive that

$$
\begin{aligned}
&\|\|\left(\underline{5.27)}\left\|_{L_{v}^{1+\delta}}\right\|_{L_{x}^{3}}\right. \\
& \lesssim \Omega\|\| \frac{1}{w_{\tilde{\vartheta}}(v)^{\iota}} w_{\tilde{\vartheta}}(u) \alpha_{f, \varepsilon}(s, x, u)^{\beta}\left|\nabla_{v} f(s, x, u)\right|\left\|_{L_{v}^{p}}\right\|_{L_{u, x}^{p}} \\
& \lesssim\left\|\frac{1}{w_{\tilde{\vartheta}}(v)^{\iota}}\right\|_{L_{v}^{p}}\left\|w_{\tilde{\vartheta}}(u) \alpha_{f, \varepsilon}(s, x, u)^{\beta} \mid \nabla_{v} f(s, x, u)\right\| \|_{L_{u, x}^{p}} \\
& \lesssim\left\|w_{\tilde{\vartheta}} \alpha_{f, \varepsilon}^{\beta} \mid \nabla_{v} f(s)\right\| \|_{L^{p}} .
\end{aligned}
$$

Combining (5.31) and (5.32) we conclude that

$$
\|\left(\text { (5.27) }\left\|_{L_{x}^{3} L_{v}^{1+\delta}} \lesssim\right\| w_{\tilde{\vartheta}} \alpha_{f, \varepsilon}^{\beta} \nabla_{v} f(s) \|_{L_{x, v}^{p}} .\right.
$$

Finally from (5.30) and (5.33), and using the Minkowski inequality, we conclude that

$$
\begin{aligned}
& \|\left(\text { (5.14) } \|_{L_{v}^{3} L_{x}^{1+\delta}}\right. \\
\lesssim & \left(1+\left\|w_{\vartheta} f\right\|_{\infty}\right) \int_{0}^{t}\left[\left\|\nabla_{v} f(s)\right\|_{L_{x}^{3} L_{v}^{1+\delta}}+\left\|w_{\tilde{\vartheta}} \alpha_{f, \varepsilon}^{\beta}\left|\nabla_{v} f(s)\right|\right\|_{L_{x, v}^{p}}\right] \mathrm{d} s .
\end{aligned}
$$

Collecting terms from (5.11)-(5.15), and (5.19), (5.12), (5.21), (5.22), (5.34), we derive

$$
\begin{aligned}
& \sup _{0 \leq s \leq t}\left\|\nabla_{v} f(s)\right\|_{L_{x}^{3} L_{v}^{1+\delta}} \\
& \lesssim 1+\left\|w_{\tilde{\vartheta}} \nabla_{v} f(0)\right\|_{L_{x, v}^{3}}+\sup _{0 \leq s \leq t}\left\|w_{\vartheta} f(s)\right\|_{\infty}+\int_{0}^{t}\left\|w_{\tilde{\vartheta}} f(s)\right\|_{2} \\
& \quad+t\left(1+\left\|w_{\vartheta} f\right\|_{\infty}\right) \sup _{0 \leq s \leq t}\left\|w_{\tilde{\vartheta}} \alpha_{f, \varepsilon}^{\beta} \nabla_{x, v} f(s)\right\|_{p} \\
& \quad+\left(1+\left\|w_{\vartheta} f\right\|_{\infty}\right) \int_{0}^{t}\left\|\nabla_{v} f(s)\right\|_{L_{x}^{3} L_{v}^{1+\delta}},
\end{aligned}
$$

where the first two lines of RHS is bounded due to the assumptions (1.36), (1.37). By the Gronwall inequality we prove (5.2). 


\section{Local Existence}

Theorem 7. Let $0<\tilde{\vartheta}<\vartheta \ll 1$. Assume that for sufficiently small $M>0, F_{0}=\mu+\sqrt{\mu} f_{0} \geq 0$, and

$$
\left\|w_{\vartheta} f_{0}\right\|_{\infty} \leq \frac{M}{2}
$$

and $\left\|w_{\tilde{\vartheta}} \alpha_{f_{0}, \varepsilon}^{\beta} \nabla_{x, v} f_{0}\right\|_{p}<\infty$ for $0<\varepsilon \ll 1$ and (1.32), where $(p, \beta)$ satisfies 4.1), and $\left\|w_{\tilde{\vartheta}} \nabla_{v} f_{0}\right\|_{L_{x, v}^{3}}<+\infty$.

Then there exists $T^{*}(M)>0$ and a unique solution $F(t, x, v)=\mu+\sqrt{\mu} f(t, x, v) \geq 0$ to (1.1), (1.5), (1.8), (1.9), and (1.10) in $\left[0, T^{*}(M)\right) \times \Omega \times \mathbb{R}^{3}$ such that

$$
\sup _{0 \leq t \leq T *}\left\|w_{\vartheta} f(t)\right\|_{\infty} \leq M
$$

Moreover

$$
\sup _{0 \leq t \leq T^{*}}\left\|\nabla_{v} f(t)\right\|_{L_{x}^{3} L_{v}^{1+\delta}}<\infty \text { for } 0<\delta \ll 1
$$

and

$$
\sup _{0 \leq t \leq T *}\left\{\left\|w_{\tilde{\vartheta}} \alpha_{f, \varepsilon}^{\beta} \nabla_{x, v} f(t)\right\|_{p}^{p}+\int_{0}^{t}\left|w_{\tilde{\vartheta}} \alpha_{f, \varepsilon}^{\beta} \nabla_{x, v} f(t)\right|_{p,+}^{p}\right\}<\infty .
$$

Furthermore, $\left\|w_{\vartheta} f(t)\right\|_{\infty},\left\|\nabla_{v} f(t)\right\|_{L_{x}^{3} L_{v}^{1+\delta}}$ and $\left\|w_{\tilde{\vartheta}} \alpha_{f, \varepsilon}^{\beta} \nabla_{x, v} f(t)\right\|_{p}^{p}$, and $\int_{0}^{t}\left|w_{\tilde{\vartheta}} \alpha_{f, \varepsilon}^{\beta} \nabla_{x, v} f(t)\right|_{p,+}^{p}$ are continuous in $t$.

Proof. Within the whole proof of Theorem 7 we consider a sequence for $\ell \geq 0$

$$
\begin{array}{r}
\partial_{t} F^{\ell+1}+v \cdot \nabla_{x} F^{\ell+1}-\nabla \phi^{\ell} \cdot \nabla_{v} F^{\ell+1} \\
=Q_{\text {gain }}\left(F^{\ell}, F^{\ell}\right)-Q_{\text {loss }}\left(F^{\ell}, F^{\ell+1}\right), \\
-\Delta \phi^{\ell}=\int_{\mathbb{R}^{3}} F^{\ell} \mathrm{d} v-\rho_{0}, \quad \int_{\Omega} \phi^{\ell} \mathrm{d} x=0,\left.\quad \frac{\partial \phi^{\ell}}{\partial n}\right|_{\partial \Omega}=0,
\end{array}
$$

and, on $(x, v) \in \gamma_{-}$,

$$
F^{\ell+1}(t, x, v)=c_{\mu} \mu \int_{n(x) \cdot v>0} F^{\ell}(t, x, u)\{n(x) \cdot u\} \mathrm{d} u,
$$

and $F^{\ell+1}(0, x, v)=F_{0}(x, v)$ for $\ell \geq 0$. We set $F^{0}(t, x, v) \equiv \mu$ and $\phi^{0} \equiv 0$.

Step 1. We claim that

We define

$$
\text { If } 0 \leq F^{\ell} \text {, then } 0 \leq F^{\ell+1} \text {. }
$$

$$
\nu(F):=\iint_{\mathbb{R}^{3} \times \mathbb{S}^{2}}|(v-u) \cdot \omega| F(u) \mathrm{d} \omega \mathrm{d} u .
$$

Clearly we have

$$
\nu\left(F^{\ell}\right) \geq 0 \quad \text { and } \quad Q_{\text {gain }}\left(F^{\ell}, F^{\ell}\right) \geq 0 \quad \text { and }\left.\quad F^{\ell+1}\right|_{\gamma_{-}} \geq 0 .
$$

Denote the characteristics $\left(X^{\ell}, V^{\ell}\right)$ which solves

$$
\begin{aligned}
& \frac{d}{d s} X^{\ell}(s ; t, x, v)=V^{\ell}(s ; t, x, v) \\
& \frac{d}{d s} V^{\ell}(s ; t, x, v)=-\nabla \phi^{\ell}\left(s, X^{\ell}(s ; t, x, v)\right) .
\end{aligned}
$$

Note that it is well-known that $\nabla \phi^{\ell}$ in (6.4) is quasi-Lipschitz continuous and (6.9) induces a Hölder continuous characteristics $\left(X^{\ell}(s ; t, x, v), V^{\ell}(s ; t, x, v)\right)$. (see Chapter 8 of [31] for example)

From (6.4), for $t-t_{\mathbf{b}}(t, x, v) \leq s \leq t$,

$$
\begin{aligned}
& \frac{d}{d s}\left\{e^{-\int_{s}^{t} \nu\left(F^{\ell}\right)\left(\tau, X^{\ell}(\tau), V^{\ell}(\tau)\right) \mathrm{d} \tau} F^{\ell+1}\left(s, X^{\ell}(s), V^{\ell}(s)\right)\right\} \\
= & e^{-\int_{s}^{t} \nu\left(F^{\ell}\right)\left(\tau, X^{\ell}(\tau), V^{\ell}(\tau)\right) \mathrm{d} \tau} Q_{\text {gain }}\left(F^{\ell}, F^{\ell}\right)\left(s, X^{\ell}(s), V^{\ell}(s)\right),
\end{aligned}
$$

where $\left(X^{\ell}(s), V^{\ell}(s)\right):=\left(X^{\ell}(s ; t, x, v), V^{\ell}(s ; t, x, v)\right)$. From the representation along the trajectory and (6.8), we prove that $F^{\ell+1} \geq 0$, and hence (6.6).

Step 2-1. We prove, from Step 2-1 to Step 2-3, that by choosing $M \ll 1$ and $T^{*}=T^{*}(M) \ll 1$ and

$$
\sup _{0 \leq t \leq T^{*}} \max _{\ell}\left\|w_{\vartheta} f^{\ell}(t)\right\|_{\infty} \leq M
$$


We define

$$
h^{\ell}(t, x, v):=w_{\vartheta}(v) f^{\ell}(t, x, v) .
$$

By an induction hypothesis we assume

$$
\sup _{0 \leq t \leq T^{*}}\left\|h^{\ell}(t)\right\|_{\infty} \leq M .
$$

Then $h^{\ell+1}$ solves

$$
\begin{aligned}
& {\left[\partial_{t}+v \cdot \nabla_{x}-\nabla_{x} \phi^{\ell} \cdot \nabla_{v}+\nu+\frac{v}{2} \cdot \nabla \phi^{\ell}-\frac{\nabla_{x} \phi^{\ell} \cdot \nabla_{v} w_{\vartheta}}{w_{\vartheta}}\right] h^{\ell+1} } \\
= & K_{w_{\vartheta}} h^{\ell}-v \cdot \nabla \phi^{\ell} w_{\vartheta} \sqrt{\mu}+w_{\vartheta} \Gamma_{\text {gain }}\left(\frac{h^{\ell}}{w_{\vartheta}}, \frac{h^{\ell}}{w_{\vartheta}}\right)-w_{\vartheta} \Gamma_{\text {loss }}\left(\frac{h^{\ell}}{w_{\vartheta}}, \frac{h^{\ell+1}}{w_{\vartheta}}\right), \\
& -\Delta \phi^{\ell}=\int_{\mathbb{R}^{3}} f^{\ell} \sqrt{\mu} \mathrm{d} v, \quad \int_{\Omega} \phi^{\ell} \mathrm{d} x=0,\left.\quad \frac{\partial \phi^{\ell}}{\partial n}\right|_{\partial \Omega}=0,
\end{aligned}
$$

where $K_{w_{\vartheta}}(\cdot)=w_{\vartheta} K\left(\frac{1}{w_{\vartheta}} \cdot\right)$. The boundary condition is

$$
\left.h^{\ell+1}\right|_{\gamma_{-}}=c_{\mu} w_{\vartheta} \sqrt{\mu} \int_{n \cdot u>0} h^{\ell} w_{\vartheta}^{-1} \sqrt{\mu}\{n \cdot u\} \mathrm{d} u .
$$

We define

$$
\nu^{\ell}(t, x, v):=\nu(v)+\frac{v}{2} \cdot \nabla \phi^{\ell}-\frac{\nabla_{x} \phi^{\ell} \cdot \nabla_{v} w_{\vartheta}}{w_{\vartheta}}+\nu\left(\sqrt{\mu} \frac{h^{\ell}}{w_{\vartheta}}\right) .
$$

From (6.13), for $M \ll 1,\left\|\nabla \phi^{\ell}\right\|_{\infty} \ll 1$ and hence

Let

$$
\nu^{\ell}(t, x, v) \geq \frac{\nu_{0}}{2}\langle v\rangle .
$$

Note that

$$
g^{\ell}:=-v \cdot \nabla \phi^{\ell} \sqrt{\mu}+\Gamma_{\text {gain }}\left(\frac{h^{\ell}}{w_{\vartheta}}, \frac{h^{\ell}}{w_{\vartheta}}\right) .
$$

where we have used

$$
\left|w_{\vartheta} g^{\ell}\right| \lesssim\left\|h^{\ell}\right\|_{\infty}+\langle v\rangle\left\|h^{\ell}\right\|_{\infty}^{2}
$$

Along the trajectory from (6.14)

$$
\left|w_{\vartheta} \Gamma\left(\frac{h}{w_{\vartheta}}, \frac{h}{w_{\vartheta}}\right)\right| \lesssim\langle v\rangle\|h\|_{\infty}^{2} .
$$

$$
\begin{aligned}
& \frac{d}{d s}\left\{e^{-\int_{s}^{t} \nu^{\ell}\left(\tau, X^{\ell}(\tau), V^{\ell}(\tau)\right) \mathrm{d} \tau} h^{\ell+1}\left(s, X^{\ell}(s ; t, x, v), V^{\ell}(s ; t, x, v)\right)\right\} \\
= & e^{-\int_{s}^{t} \nu^{\ell}\left(\tau, X^{\ell}(\tau), V^{\ell}(\tau)\right) \mathrm{d} \tau} \\
& \times\left\{K_{w_{\vartheta}} h^{\ell}\left(s, X^{\ell}(s), V^{\ell}(s)\right)+w_{\vartheta} g^{\ell}\left(s, X^{\ell}(s), V^{\ell}(s)\right)\right\} .
\end{aligned}
$$

By (6.15) and (6.19) we represent $h^{\ell+1}$ along the stochastic cycles:

$$
\begin{aligned}
& t_{1}^{\ell}(t, x, v):=\sup \left\{s<t: X^{\ell}(s ; t, x, v) \in \partial \Omega\right\} \\
& x_{1}^{\ell}(t, x, v):=X^{\ell}\left(t_{1}^{\ell}(t, x, v) ; t, x, v\right), \\
& t_{2}^{\ell-1}\left(t, x, v, v_{1}\right) \\
& \quad:=\sup \left\{s<t_{1}^{\ell}: X^{\ell-1}\left(s ; t_{1}^{\ell}(t, x, v), x_{1}^{\ell}(t, x, v), v_{1}\right) \in \partial \Omega\right\}, \\
& x_{2}^{\ell-1}\left(t, x, v, v_{1}\right):=X^{\ell-1}\left(t_{2}^{\ell-1}\left(t, x, v, v_{1}\right) ; t_{1}^{\ell}(t, x, v), x_{1}^{\ell}(t, x, v), v_{1}\right),
\end{aligned}
$$

and inductively

$$
\begin{aligned}
& t_{k}^{\ell-(k-1)}\left(t, x, v, v_{1}, \cdots, v_{k-1}\right) \\
& :=\sup \left\{s<t_{k-1}^{\ell-(k-2)}: X^{\ell-1}\left(s ; t_{k-1}^{\ell-(k-2)}, x_{k-1}^{\ell-(k-2)}, v_{k-1}\right) \in \partial \Omega\right\}, \\
& x_{k}^{\ell-(k-1)}\left(t, x, v, v_{1}, \cdots, v_{k-1}\right) \\
& :=X^{\ell-(k-2)}\left(t_{k}^{\ell-(k-1)} ; t_{k-1}^{\ell-(k-2)}, x_{k-1}^{\ell-(k-2)}, v_{k-1}\right) .
\end{aligned}
$$


Here,

$$
\begin{aligned}
t_{i}^{\ell-(i-1)} & :=t_{i}^{\ell-(i-1)}\left(t, x, v, v_{1}, \cdots, v_{i-1}\right), \\
x_{i}^{\ell-(i-1)} & :=x_{i}^{\ell-(i-1)}\left(t, x, v, v_{1}, \cdots, v_{i-1}\right) .
\end{aligned}
$$

From (6.21) and (6.15), we have

$$
\begin{aligned}
& h^{\ell+1}(t, x, v) \\
= & \mathbf{1}_{t_{1}^{\ell} \leq 0} e^{-\int_{0}^{t} \nu^{\ell}} h^{\ell+1}\left(0, X^{\ell}(0), V^{\ell}(0)\right) \\
& +\int_{\max \left\{t_{1}^{\ell}, 0\right\}}^{t} e^{-\int_{s}^{t} \nu^{\ell}}\left|\left[K_{w_{\vartheta}} h^{\ell}+w_{\vartheta} g^{\ell}\right]\left(s, X^{\ell}(s ; t, x, v), V^{\ell}(s ; t, x, v)\right)\right| \mathrm{d} s \\
& +\mathbf{1}_{t_{1}^{\ell} \geq 0} e^{-\int_{t_{1}^{\ell}}^{t} \nu^{\ell}} h^{\ell+1}\left(t_{1}^{\ell}, X^{\ell}\left(t_{1}^{\ell} ; t, x, v\right), V^{\ell}\left(t_{1}^{\ell} ; t, x, v\right)\right) .
\end{aligned}
$$

We define

$$
\tilde{w}_{\vartheta}(v) \equiv \frac{1}{w_{\vartheta}(v) \sqrt{\mu(v)}} .
$$

From (6.15),

$$
\begin{aligned}
& \text { the last line of (6.24) } \\
& =\mathbf{1}_{t_{1}^{\ell} \geq 0} e^{-\int_{t_{1}^{\ell}}^{t} \nu^{\ell}} \frac{1}{\tilde{w}_{\vartheta}\left(V^{\ell}\left(t_{1}^{\ell}\right)\right)} \int_{n\left(x_{1}^{\ell}\right) \cdot v_{1}>0} h^{\ell}\left(t_{1}^{\ell}, x_{1}^{\ell}, v_{1}\right) \tilde{w}_{\vartheta}\left(v_{1}\right) c_{\mu} \mu\left\{n\left(x_{1}^{\ell}\right) \cdot v_{1}\right\} \mathrm{d} v_{1} .
\end{aligned}
$$

We define $\mathcal{V}(x)=\left\{v \in \mathbb{R}^{3}: n(x) \cdot v>0\right\}$ with a probability measure $\mathrm{d} \sigma=\mathrm{d} \sigma(x)$ on $\mathcal{V}(x)$ which is given by

$$
\mathrm{d} \sigma \equiv c_{\mu} \mu(v)\{n(x) \cdot v\} \mathrm{d} v .
$$

Let

$$
\mathcal{V}_{j}:=\left\{v_{j} \in \mathbb{R}^{3}: n\left(x_{j}^{\ell-(j-1)}\right) \cdot v_{j}>0\right\} .
$$

Then inductively we obtain from (6.24), (6.21) and (6.15),

$$
\begin{aligned}
& \left|h^{\ell+1}(t, x, v)\right| \\
\leq & \mathbf{1}_{t_{1}^{\ell} \leq 0} e^{-\int_{0}^{t} \nu^{\ell}}\left|h^{\ell+1}\left(0, X^{\ell}(0), V^{\ell}(0)\right)\right| \\
& +\int_{\max \left\{t_{1}^{\ell}, 0\right\}}^{t} e^{-\int_{s}^{t} \nu^{\ell}}\left|\left[K_{w_{\vartheta}} h^{\ell}+w_{\vartheta} g^{\ell}\right]\left(s, X^{\ell}(s ; t, x, v), V^{\ell}(s ; t, x, v)\right)\right| \mathrm{d} s \\
& +\mathbf{1}_{t_{1}^{\ell}>0} \frac{e^{-\int_{t_{1}^{\ell}}^{t} \nu^{\ell}}}{\tilde{w}_{\vartheta}\left(V^{\ell}\left(t_{1}^{\ell}\right)\right)} \int_{\prod_{j=1}^{k-1} \mathcal{V}_{j}}|H|,
\end{aligned}
$$

where $|H|$ is bounded by

$$
\begin{aligned}
& \sum_{l=1}^{k-1} \mathbf{1}_{\left\{t_{l+1}^{\ell-l} \leq 0<t_{l}^{\ell-(l-1)}\right\}}\left|h^{\ell-l}\left(0, X^{\ell-l}\left(0 ; v_{l}\right), V^{\ell-l}\left(0 ; v_{l}\right)\right)\right| \mathrm{d} \Sigma_{l}(0) \\
& +\sum_{l=1}^{k-1} \int_{\max \left\{t_{l+1}^{\ell-l}, 0\right\}}^{t_{l}^{\ell-(l-1)}} \mathbf{1}_{\left\{t_{l+1}^{\ell-l} \leq 0<t_{l}^{\ell-(l-1)}\right\}} \\
& \quad \times \mid\left[K_{w_{\vartheta}} h^{\ell-l}+w_{\vartheta} g^{\ell-l}\right]\left(s, X^{\ell-l}\left(s ; v_{l}\right), V^{\ell-l}\left(s ; v_{l}\right) \mid \mathrm{d} \Sigma_{l}(s) \mathrm{d} s\right. \\
& +\mathbf{1}_{\left\{0<t_{k}^{\ell-(k-1)}\right\}}\left|h^{\ell-(k-1)}\left(t_{k}^{\ell-(k-1)}, x_{k}^{\ell-(k-1)}, v_{k-1}\right)\right| \mathrm{d} \Sigma_{k-1}\left(t_{k}^{\ell-(k-1)}\right),
\end{aligned}
$$

with

$$
\begin{aligned}
d \Sigma_{l}^{k-1}(s)=\left\{\Pi_{j=l+1}^{k-1} \mathrm{~d} \sigma_{j}\right\} & \times\left\{e^{-\int_{s}^{t_{l}} \nu_{\phi}} \tilde{w}_{\vartheta}\left(v_{l}\right) \mathrm{d} \sigma_{l}\right\} \\
& \times \Pi_{j=1}^{l-1}\left\{e^{-\int_{t_{j+1}}^{t_{j}} \nu_{\phi}} \mathrm{d} \sigma_{j}\right\},
\end{aligned}
$$

and

$$
\begin{aligned}
& X^{\ell-l}\left(s ; v_{l}\right):=X^{\ell-l}\left(s ; t_{l}^{\ell-(l-1)}, x_{l}^{\ell-(l-1)}, v_{l}\right), \\
& V^{\ell-l}\left(s ; v_{l}\right):=V^{\ell-l}\left(s ; t_{l}^{\ell-(l-1)}, x_{l}^{\ell-(l-1)}, v_{l}\right) .
\end{aligned}
$$


Step 2-2. We claim that there exists $T>0$ and $k_{0}>0$ such that for all $k \geq k_{0}$ and for all $(t, x, v) \in$ $[0, T] \times \bar{\Omega} \times \mathbb{R}^{3}$, we have

$$
\int_{\prod_{j=1}^{k-1} \mathcal{V}_{j}} \mathbf{1}_{\left\{t^{k}\left(t, x, v, v^{1}, \cdots, v^{k-1}\right)>0\right\}} \mathrm{d} \Sigma_{k-1}^{k-1} \lesssim \Omega\left\{\frac{1}{2}\right\}^{k / 5}
$$

The proof of the claim is a modification of a proof of Lemma 14 of [15].

For $0<\delta \ll 1$ we define

$$
\mathcal{V}_{j}^{\delta}:=\left\{v^{j} \in \mathcal{V}^{j}:\left|v^{j} \cdot n\left(x^{j}\right)\right|>\delta,\left|v^{j}\right| \leq \delta^{-1}\right\}
$$

Choose

$$
T=\frac{2}{\delta^{2 / 3}\left(1+\|\nabla \phi\|_{\infty}\right)^{2 / 3}} .
$$

We claim that

$$
\left|t^{j}-t^{j+1}\right| \gtrsim \delta^{3}, \text { for } v^{j} \in \mathcal{V}_{j}^{\delta}, 0 \leq t \leq T, 0 \leq t^{j}
$$

For $j \geq 1$

$$
\begin{aligned}
& \left|\int_{t^{j}}^{t^{j+1}} V\left(s ; t^{j}, x^{j}, v^{j}\right) \mathrm{d} s\right|^{2} \\
= & \left|x^{j+1}-x^{j}\right|^{2} \\
\gtrsim & \left|\left(x^{j+1}-x^{j}\right) \cdot n\left(x^{j}\right)\right| \\
= & \left|\int_{t^{j}}^{t^{j+1}} V\left(s ; t^{j}, x^{j}, v^{j}\right) \cdot n\left(x^{j}\right) \mathrm{d} s\right| \\
= & \left|\int_{t^{j}}^{t^{j+1}}\left(v^{j}-\int_{t^{j}}^{s} \nabla \phi\left(\tau, X\left(\tau ; t^{j}, x^{j}, v^{j}\right)\right) \mathrm{d} \tau\right) \cdot n\left(x^{j}\right) \mathrm{d} s\right| \\
\geq & \left|v^{j} \cdot n\left(x^{j}\right)\right|\left|t^{j}-t^{j+1}\right|-\left|\int_{t^{j}}^{t^{j+1}} \int_{t^{j}}^{s} \nabla \phi\left(\tau, X\left(\tau ; t^{j}, x^{j}, v^{j}\right)\right) \cdot n\left(x^{j}\right) \mathrm{d} \tau \mathrm{d} s\right| .
\end{aligned}
$$

Here we have used the fact if $x, y \in \partial \Omega$ and $\partial \Omega$ is $C^{2}$ and $\Omega$ is bounded then $|x-y|^{2} \gtrsim \Omega|(x-y) \cdot n(x)|$. Hence

$$
\begin{aligned}
& \left|v^{j} \cdot n\left(x^{j}\right)\right| \\
& \lesssim \frac{1}{\left|t^{j}-t^{j+1}\right|}\left|\int_{t^{j}}^{t^{j+1}} V\left(s ; t^{j}, x^{j}, v^{j}\right) \mathrm{d} s\right|^{2} \\
& \quad+\frac{1}{\left|t^{j}-t^{j+1}\right|}\left|\int_{t^{j}}^{t^{j+1}} \int_{t^{j}}^{s} \nabla \phi\left(\tau, X\left(\tau ; t^{j}, x^{j}, v^{j}\right)\right) \cdot n\left(x^{j}\right) \mathrm{d} \tau \mathrm{d} s\right| \\
& \lesssim\left|t^{j}-t^{j+1}\right|\left\{\left|v^{j}\right|^{2}+\left|t^{j}-t^{j+1}\right|^{3}\|\nabla \phi\|_{\infty}^{2}\right. \\
& \left.\quad+\frac{1}{2} \sup _{t^{j+1} \leq \tau \leq t^{j}}\left|\nabla \phi\left(\tau, X\left(\tau ; t^{j}, x^{j}, v^{j}\right)\right) \cdot n\left(x^{j}\right)\right|\right\} .
\end{aligned}
$$

For $v^{j} \in \mathcal{V}_{j}^{\delta}, 0 \leq t \leq T$, and $t^{j} \geq 0$

$$
\left|v^{j} \cdot n\left(x^{j}\right)\right| \lesssim\left|t^{j}-t^{j+1}\right|\left\{\delta^{-2}+T^{3}\|\nabla \phi\|_{\infty}^{2}+\|\nabla \phi\|_{\infty}\right\}
$$

We choose $T$ as (6.36) then prove (6.37).

Therefore if $t^{k} \geq 0$ then there can be at most $\left\{\left[\frac{C_{\Omega}}{\delta^{3}}\right]+1\right\}$ numbers of $v^{m} \in \mathcal{V}_{m}^{\delta}$ for $1 \leq m \leq k-1$. Equivalently there are at least $k-2-\left[\frac{C_{\Omega}}{\delta^{3}}\right]$ numbers of $v^{i} \in \mathcal{V}_{i} \backslash \mathcal{V}_{i}^{\delta}$ for $0 \leq i \leq m$. 
Let us choose $k=N \times\left(\left[\frac{C_{\Omega}}{\delta^{3}}\right]+1\right)$ and $N=\left(\left[\frac{C_{\Omega}}{\delta^{3}}\right]+1\right) \gg C>1$. Then we have

$$
\begin{aligned}
& \int_{\prod_{j=1}^{k-1} \mathcal{V}_{j}} 1_{\left\{t^{k}\left(t, x, v, v^{1}, \cdots, v^{k-1}\right)>0\right\}} d \Sigma_{k-1}^{k-1} \\
\leq & \sum_{m=1}^{\left[\frac{C_{\Omega}}{\delta^{3}}\right]+1} \int\left\{\begin{array}{c}
\text { there are exactly } m \text { of } v_{i} \in \mathcal{V}_{i}^{\delta} \\
\text { and } k-1-m \text { of } v_{i} \in \mathcal{V}_{i} \backslash \mathcal{V}_{i}^{\delta}
\end{array}\right\} \prod_{j=1}^{k-1} C_{0} \mu\left(v^{j}\right)^{1 / 4} \mathrm{~d} v^{j} \\
\leq & \sum_{m=1}^{\left[\frac{C_{\Omega}}{\delta^{3}}\right]+1}\left(\begin{array}{c}
k-1 \\
m
\end{array}\right)\left\{\int_{\mathcal{V}} C_{0} \mu(v)^{1 / 4} \mathrm{~d} v\right\}^{m}\left\{\int_{\mathcal{V} \backslash \mathcal{V}^{\delta}} C_{0} \mu(v)^{1 / 4} \mathrm{~d} v\right\}^{k-1-m} \\
\leq & \left(\left[\frac{C_{\Omega}}{\delta^{3}}\right]+1\right)\{k-1\}^{\left[\frac{C_{\Omega}}{\delta^{3}}\right]+1}\{\delta\}^{k-2-\left[\frac{C_{\Omega}}{\delta^{3}}\right]}\left\{\int_{\mathcal{V}} C_{0} \mu(v)^{1 / 4} d v\right\}^{\left[\frac{C_{\Omega}}{\delta^{3}}\right]+1} \\
\leq & \{C N\}^{\frac{k}{N}}\left\{\frac{k}{N}\right\}^{\frac{k}{N}}\left\{\frac{k}{N}\right\}^{-\frac{k}{N} \frac{N^{2}}{20}} \\
\leq & \left\{\frac{k}{N}\right\}^{\frac{k}{N}\left(-\frac{N^{2}}{20}+3\right)} \\
\leq & \left\{\frac{1}{2}\right\}^{k},
\end{aligned}
$$

where we have chosen $k=N \times\left(\left[\frac{C_{\Omega}}{\delta^{3}}\right]+1\right)$ and $N=\left(\left[\frac{C_{\Omega}}{\delta^{3}}\right]+1\right) \gg C>1$.

Step 2-3. From (6.19), (6.17), and (6.28)-(6.32), and (6.34), if we choose $\ell \geq k_{0}$ and $0 \leq t \leq T$ where $k_{0}$ and $T$ in 6.34

$$
\begin{aligned}
& \left|h^{\ell+1}(t, x, v)\right| \\
& \leq C_{k}\left\|e^{-\frac{\nu_{0}}{2} t} h_{0}\right\|_{\infty} \\
& +\int_{\max \left\{t_{1}^{\ell}, 0\right\}}^{t} e^{-\frac{\nu_{0}}{2}(t-s)} \int_{\mathbb{R}^{3}} \mathbf{k}_{\varrho}\left(V^{\ell}(s), u\right)\left|h^{\ell}\left(s, X^{\ell}(s), u\right)\right| \mathrm{d} u \mathrm{~d} s \\
& +C_{k} \sup _{l} \int_{\max \left\{t_{l+1}^{\ell-1}, 0\right\}}^{t_{l}^{\ell-(l-1)}} e^{-\frac{\nu_{0}}{2}(t-s)} \int_{\mathbb{R}^{3}} \int_{\mathbb{R}^{3}} \mathbf{k}_{\varrho}\left(V^{\ell-l}\left(s ; v_{l}\right), u\right) \\
& \times\left|h^{\ell-l}\left(s, X^{\ell-l}\left(s ; v_{l}\right), u\right)\right|\left\{n\left(x_{l}\right) \cdot v_{l}\right\} \frac{\sqrt{\mu\left(v_{l}\right)}}{w_{\vartheta}\left(v_{l}\right)} \mathrm{d} v_{l} \mathrm{~d} u \mathrm{~d} s \\
& +\int_{\max \left\{t_{1}^{\ell}, 0\right\}}^{t}\left\langle V^{\ell}(s ; t, x, v)\right\rangle e^{-\int_{s}^{t} \frac{\nu^{\ell}(\tau)}{2} \mathrm{~d} \tau}\left\|e^{-\frac{\nu_{0}}{2}(t-s)} h^{\ell}(s)\right\|_{\infty}^{2} \mathrm{~d} s \\
& +C_{k} \sup _{l} \int_{\max \left\{t_{l+1}^{\ell-l}, 0\right\}}^{t_{l}^{\ell-(l-1)}}\left\langle V^{\ell-l}\left(s ; v_{l}\right)\right\rangle
\end{aligned}
$$

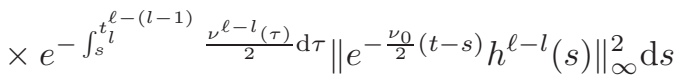

$$
\begin{aligned}
& +\int_{\max \left\{t_{1}^{\ell}, 0\right\}}^{t}\left\|e^{-\frac{\nu_{0}}{2}(t-s)} \nabla \phi^{\ell}(s)\right\|_{\infty} \mathrm{d} s \\
& +C_{k} \sup _{l} \int_{\max \left\{t_{l+1}^{\ell-l}, 0\right\}}^{t_{l}^{\ell-(l-1)}}\left\|e^{-\frac{\nu_{0}}{2}(t-s)} \nabla \phi^{\ell-l}(s)\right\|_{\infty} \mathrm{d} s \\
& +\left\{\frac{1}{2}\right\}^{k / 5}\left\|e^{-\frac{\nu_{0}}{2}\left(t-t_{k}^{\ell-(k-1)}\right)} h\left(t_{k}^{\ell-(k-1)}\right)\right\|_{\infty},
\end{aligned}
$$

where we used the abbreviation of (6.33). 
From $\int_{0}^{t}\left\langle V^{\ell-l}\left(s ; v_{l}\right)\right\rangle e^{-\int_{s}^{t_{l}^{\ell-(l-1)}} \frac{\nu^{\ell-l}(\tau)}{2} \mathrm{~d} \tau} \mathrm{d} s \lesssim 1$ and (4.14), we derive that

$$
\begin{aligned}
\left\|h^{\ell+1}(t)\right\|_{\infty} \lesssim_{k} & \|h(0)\|_{\infty}+o(1)\left\|h\left(t_{k}^{\ell-(k-1)}\right)\right\|_{\infty} \\
& +t \max _{l \geq 0} \sup _{0 \leq s \leq t}\left\|h^{\ell-l}(s)\right\|_{\infty}+\max _{l \geq 0} \sup _{0 \leq s \leq t}\left\|h^{\ell-l}(s)\right\|_{\infty}^{2} .
\end{aligned}
$$

By taking supremum in $\ell$ and choosing $M \ll 1$ and $0 \leq t \leq T^{*} \leq T$ with $T^{*} \ll 1$, we conclude (6.11).

Step 3. We claim that there exist $T^{* *} \ll 1$ (and $T^{* *}<T^{*}(M)$ ) and $C>0$ such that the sequence $F^{\ell}=\mu+\sqrt{\mu} f^{\ell}$ in (6.4) satisfies

$$
\max _{\ell \geq 0} \sup _{0 \leq t \leq T^{* *}} \mathcal{E}^{\ell}(t) \leq C\left\{\left\|w_{\tilde{\vartheta}} f_{0}\right\|_{p}^{p}+\left\|w_{\tilde{\vartheta}} \alpha_{f_{0}, \varepsilon}^{\beta} \nabla_{x, v} f_{0}\right\|_{p}^{p}\right\}<\infty .
$$

where we define, for $0<\varepsilon \ll 1$,

$$
\begin{aligned}
\mathcal{E}^{\ell+1}(t):= & \left\|w_{\tilde{\vartheta}} f^{\ell+1}(t)\right\|_{p}^{p}+\left\|w_{\tilde{\vartheta}} \alpha_{f^{\ell}, \varepsilon}^{\beta} \nabla_{x, v} f^{\ell+1}(t)\right\|_{p}^{p} \\
& +\int_{0}^{t}\left|w_{\tilde{\vartheta}} \alpha_{f^{\ell}, \varepsilon}^{\beta} \nabla_{x, v} f^{\ell+1}(t)\right|_{p,+}^{p} .
\end{aligned}
$$

It suffices to prove the following induction statement: there exist $T^{* *} \ll 1$ (and $\left.T^{* *}<T^{*}(M)\right)$ and $C>0$ such that

$$
\text { if } \begin{array}{r}
\max _{0 \leq m \leq \ell} \sup _{0 \leq t \leq T^{* *}} \mathcal{E}^{m}(t) \leq C\left\{\left\|w_{\tilde{\vartheta}} f_{0}\right\|_{p}^{p}+\left\|w_{\tilde{\vartheta}} \alpha_{f_{0}, \varepsilon}^{\beta} \nabla_{x, v} f_{0}\right\|_{p}^{p}\right\}<\infty \\
\text { then } \sup _{0 \leq t \leq T^{* *}} \mathcal{E}^{\ell+1}(t) \leq C\left\{\left\|w_{\tilde{\vartheta}} f_{0}\right\|_{p}^{p}+\left\|w_{\tilde{\vartheta}} \alpha_{f_{0}, \varepsilon}^{\beta} \nabla_{x, v} f_{0}\right\|_{p}^{p}\right\} .
\end{array}
$$

The proof is similar to the proof of Proposition 4. By taking derivatives $\partial \in\left\{\nabla_{x}, \nabla_{v}\right\}$ to $f^{\ell+1}=\left\{F^{\ell+1}-\right.$ $\mu\} / \sqrt{\mu}$ to 6.4

$$
\left[\partial_{t}+v \cdot \nabla_{x}-\nabla \phi^{\ell} \cdot \nabla_{v}+\nu+\frac{v}{2} \cdot \nabla \phi^{\ell}\right] \partial f^{\ell+1}=\mathcal{G}^{\ell+1}
$$

where $\mathcal{G}^{\ell+1}$ equals

$$
\begin{aligned}
\mathcal{G}^{\ell+1}= & -\partial v \cdot \nabla_{x} f^{\ell+1}+\partial \nabla \phi^{\ell} \cdot \nabla_{v} f^{\ell+1} \\
& +\partial\left\{\Gamma\left(f^{\ell}, f^{\ell}\right)+\Gamma_{\operatorname{loss}}\left(f^{\ell}, f^{\ell}\right)-\Gamma_{\operatorname{loss}}\left(f^{\ell}, f^{\ell+1}\right)\right\} \\
& -\partial\left[\nu(v)+\frac{v}{2} \cdot \nabla \phi^{\ell}(t, x)\right] f^{\ell+1}-\partial K f^{\ell}-\partial\left(v \cdot \nabla_{x} \phi^{\ell} \sqrt{\mu}\right) .
\end{aligned}
$$

The boundary condition is given by (1.17) but replacing $f$ in the LHS by $f^{\ell+1}$ and $f$ in the RHS by $f^{\ell}$.

Recall (4.41). From (6.11) we have $\nu_{\phi^{\ell}} \geq \frac{\nu(v)}{2}$. We can follow the proof of (4.44), (4.48), (4.53) from Lemma 6. and (4.26), (4.25), (4.23), (4.24) to obtain

$$
\begin{aligned}
& \mathcal{E}^{\ell+1}(s) \lesssim\left\|w_{\tilde{\vartheta}} \alpha_{f^{\ell}, \varepsilon}^{\beta} \nabla_{x, v} f_{0}\right\|_{p}^{p}+\underbrace{\int_{\text {boundary }}^{t}\left|w_{\tilde{\vartheta}} \alpha_{f^{\ell}, \varepsilon}^{\beta} \nabla_{x, v} f^{\ell+1}\right|_{p,-}^{p}}_{6.46} \\
& +t\left(1+\left\|w_{\vartheta} f^{\ell}\right\|_{\infty}+\left\|w_{\vartheta} f^{\ell+1}\right\|_{\infty}+\left\|\nabla^{2} \phi^{\ell}\right\|_{\infty}\right) \\
& \times \max _{m=\ell, \ell+1} \sup _{0 \leq s \leq t} \mathcal{E}^{m}(s) .
\end{aligned}
$$

There is only one extra term to (4.40) which is $\partial\left\{\Gamma_{\operatorname{loss}}\left(f^{\ell}, f^{\ell}\right)-\Gamma_{\text {loss }}\left(f^{\ell}, f^{\ell+1}\right)\right\}$ in (6.45). Its contribution in $(4.44) \mathcal{G}$ can be controlled by

$$
\begin{aligned}
& \left(1+\sup _{0 \leq s \leq t}\left\|w_{\vartheta} f^{\ell}(s)\right\|_{\infty}+\sup _{0 \leq s \leq t}\left\|w_{\vartheta} f^{\ell+1}(s)\right\|_{\infty}\right) \\
& \times \int_{0}^{t} \iint_{\Omega \times \mathbb{R}^{3}}\left|\alpha_{f^{\ell}, \varepsilon}^{\beta} w_{\tilde{\vartheta}} \partial f^{\ell+1}(v)\right|^{p-1} \\
& \times \int_{\mathbb{R}^{3}} \alpha_{f^{\ell}, \varepsilon}(v)^{\beta} \mathbf{k}_{\varrho}(v, u) w_{\tilde{\vartheta}}(v)\left\{\left|\partial f^{\ell}(u)\right|+\left|\partial f^{\ell+1}(u)\right|\right\} \mathrm{d} u \mathrm{~d} v \mathrm{~d} x \mathrm{~d} s
\end{aligned}
$$


and then further bounded as, by the way to treat (4.46) and (4.47),

$$
\sup _{0 \leq s \leq t} \max _{m=\ell, \ell+1}\left\|w_{\vartheta} f^{m}(s)\right\|_{\infty} \int_{0}^{t} \max _{m=\ell, \ell+1}\left\|\nu_{\phi_{f} \ell, w_{\tilde{\vartheta}}}^{1 / p} w_{\tilde{\vartheta}} \alpha_{f^{\ell}, \varepsilon}^{\beta} \partial f^{\ell+1}\right\|_{p}^{p} .
$$

Clearly it is bounded the RHS of (6.46).

Now we focus on (6.46) boundary. Note that $\alpha_{f^{\ell}, \varepsilon}=|n \cdot v|$ on $\gamma_{-}$. On $\gamma_{-},\left|\partial f^{\ell+1}\right|$ is bounded by (4.27) replacing $|f|,\left|\nabla_{x, v} f\right|,\left|\nabla \phi_{f}\right|$ by $\left|f^{\ell}\right|+\left|f^{\ell+1}\right|,\left|\nabla_{x, v} f^{\ell}\right|+\left|\nabla_{x, v} f^{\ell+1}\right|,\left|\nabla \phi^{\ell}\right|+\left|\nabla \phi^{\ell-1}\right|$. Now we follow Step 4 in the proof of Proposition 4. We have the same decomposition (4.64) replacing all $\nabla_{x, v} f$ by $\nabla_{x, v} f^{\ell}$. Then we obtain (4.65) but replacing all $\nabla_{x, v} f$ by $\nabla_{x, v} f^{\ell}$. Then we apply (2.54) of Lemma 7 to $\gamma_{+}(x) \backslash \gamma_{+}^{\varepsilon}(x)$ contribution in (4.64). And this leads (4.66) but replacing all $\nabla_{x, v} f, f, \phi_{f}$ by $\nabla_{x, v} f^{\ell}, f^{\ell}, \phi^{\ell-1}$. Finally we end up with

$$
\begin{aligned}
& \text { (6.46) } \text { boundary }_{\text {b }} \lesssim\left\|w_{\tilde{\vartheta}} \alpha_{f_{0}, \varepsilon}^{\beta} \nabla_{x, v} f_{0}\right\|_{p}^{p}+o(1) \max _{0 \leq m \leq \ell} \sup _{0 \leq s \leq t} \mathcal{E}^{m}(s) \\
& +t\left(1+\left\|w_{\vartheta} f^{\ell}\right\|_{\infty}+\left\|\nabla^{2} \phi^{\ell-1}\right\|_{\infty}\right) \max _{0 \leq m \leq \ell} \sup _{0 \leq s \leq t} \mathcal{E}^{m}(s) .
\end{aligned}
$$

On the other hand, from Lemma 12 ,

$$
\left\|\nabla^{2} \phi^{\ell}(t)\right\|_{\infty}+\left\|\nabla^{2} \phi^{\ell-1}(t)\right\|_{\infty} \lesssim\left[\mathcal{E}^{\ell}(t)+\mathcal{E}^{\ell-1}(t)\right]^{1 / p}
$$

From (6.46), (6.47), (6.48), and the induction hypothesis in (6.43), we derive

$$
\sup _{m \leq \ell+1} \sup _{0 \leq s \leq t} \mathcal{E}^{m}(s) \lesssim\left\|w_{\tilde{\vartheta}} \alpha_{f_{0}, \varepsilon}^{\beta} \nabla_{x, v} f_{0}\right\|_{p}^{p}+t \sup _{0 \leq s \leq t} \sup _{m \leq \ell} \mathcal{E}^{m}(s) .
$$

Finally we choose small $T^{* *}>0$ and conclude (6.43).

Step 4. Now we claim that the sequence $F^{\ell}=\mu+\sqrt{\mu} f^{\ell}$ in (6.4) satisfies

$$
\sup _{\ell} \sup _{0 \leq t \leq T^{* *}}\left\|\nabla_{v} f^{\ell}(t)\right\|_{L_{x}^{3} L_{v}^{1+\delta}} \lesssim 1
$$

To prove this, similar as above step, we claim that

$$
\begin{aligned}
& \text { if } \sup _{m \leq \ell} \sup _{m \leq t \leq T^{* *}}\left\|\nabla_{v} f^{m}(t)\right\|_{L_{x}^{3} L_{v}^{1+\delta}} \lesssim 1, \\
& \text { then } \sup _{0 \leq t \leq T^{* *}}\left\|\nabla_{v} f^{\ell+1}(t)\right\|_{L_{x}^{3} L_{v}^{1+\delta}} \lesssim 1 .
\end{aligned}
$$

Recall that $\nabla_{v} f^{\ell+1}$ solves (6.44) and (6.45) with $\partial=\nabla_{v}$. Then we follow exactly the same proof of (5.18)-(5.34) and conclude that

$$
\begin{aligned}
& \left\|\nabla_{v} f^{\ell+1}(t)\right\|_{L_{x}^{3} L_{v}^{1+\delta}} \\
& \lesssim\left\|w_{\tilde{\vartheta}} \partial_{v} f^{\ell+1}(0)\right\|_{L_{x, v}^{3}}+\sup _{0 \leq s \leq t}\left\{\left\|w_{\vartheta} f^{\ell}(s)\right\|_{\infty}+\left\|w_{\vartheta} f^{\ell+1}(s)\right\|_{\infty}\right\} \\
& +t \sup _{0 \leq s \leq t}\left\{\left\|f^{\ell}(s)\right\|_{2}+\left\|w_{\tilde{\vartheta}} \alpha_{f^{\ell-1}, \varepsilon}^{\beta} \nabla_{x, v} f^{\ell}\right\|_{p}+\left\|w_{\tilde{\vartheta}} \alpha_{f^{\ell}, \varepsilon}^{\beta} \nabla_{x, v} f^{\ell+1}\right\|_{p}\right. \\
& \left.\quad+\left\|\nabla_{v} f^{\ell}(s)\right\|_{L_{x}^{3} L_{v}^{1+\delta}}+\left\|\nabla_{v} f^{\ell+1}(s)\right\|_{L_{x}^{3} L_{v}^{1+\delta}}\right\} .
\end{aligned}
$$

From (6.43) and (6.11), we can choose $T^{* *} \ll 1$ and conclude (6.50) and hence (6.49).

Step 5. Now we claim that the convergence of whole sequence

$$
f^{\ell} \rightarrow f \text { strongly in } L^{1+}\left(\Omega \times \mathbb{R}^{3}\right) \cap L^{1+}(\gamma)
$$

Note that $f^{\ell+1}-f^{\ell}$ satisfies $\left.\left(f^{\ell+1}-f^{\ell}\right)\right|_{t=0} \equiv 0$ and (5.4) with $f=f^{\ell+1}$ and $g=f^{\ell}$ to get

$$
\begin{aligned}
& \partial_{t}\left[f^{\ell+1}-f^{\ell}\right]+v \cdot \nabla_{x}\left[f^{\ell+1}-f^{\ell}\right]-\nabla_{x} \phi_{f^{\ell}} \cdot \nabla_{v}\left[f^{\ell+1}-f^{\ell}\right] \\
& +\frac{v}{2} \cdot \nabla_{x} \phi_{f^{\ell}}\left[f^{\ell+1}-f^{\ell}\right]+\nu\left[f^{\ell+1}-f^{\ell}\right] \\
& =\nabla_{x} \phi_{f^{\ell}-f^{\ell-1}} \cdot \nabla_{v} f^{\ell-1}+K\left[f^{\ell}-f^{\ell-1}\right]-\frac{v}{2} \cdot \nabla_{x} \phi_{f^{\ell}-f^{\ell-1}} f^{\ell-1} \\
& -v \cdot \nabla_{x} \phi_{f^{\ell}-f^{\ell-1}} \sqrt{\mu}+\left\{\Gamma\left(f^{\ell}, f^{\ell}\right)-\Gamma\left(f^{\ell-1}, f^{\ell-1}\right)\right\} \\
& +\left\{\Gamma_{\text {loss }}\left(f^{\ell}, f^{\ell}\right)-\Gamma_{\text {loss }}\left(f^{\ell-1}, f^{\ell-1}\right)\right\}-\left\{\Gamma_{\text {loss }}\left(f^{\ell}, f^{\ell+1}\right)-\Gamma_{\text {loss }}\left(f^{\ell-1}, f^{\ell}\right)\right\} .
\end{aligned}
$$


With help of uniform estimatjes (6.11) and (6.49), we can modify RHSs of (5.5), (5.6), (5.7), and (5.8) in the proof of Proposition 6 to get

$$
\begin{aligned}
& \sup _{0 \leq s \leq t}\left\|f^{\ell+1}(s)-f^{\ell}(s)\right\|_{L^{1+\delta}\left(\Omega \times \mathbb{R}^{3}\right)}+\left(\int_{0}^{t}\left|f^{\ell+1}(s)-f^{\ell}(s)\right|_{L^{1+\delta}(\gamma)}\right)^{\frac{1}{1+\delta}} \\
& \lesssim t\left(1+\sup _{0 \leq s \leq t} \sup _{m}\left\|h^{m}(s)\right\|_{\infty}+\sup _{0 \leq s \leq t} \sup _{m}\left\|\nabla_{v} f^{m}(t)\right\|_{\left.L_{x}^{3} L_{v}^{1+\delta}\right)}\right. \\
& \quad \times \sup _{0 \leq s \leq t}\left\|f^{\ell}(s)-f^{\ell-1}(s)\right\|_{L^{1+\delta}\left(\Omega \times \mathbb{R}^{3}\right)} \\
& \lesssim O(t) \sup _{0 \leq s \leq t}\left\|f^{\ell}(s)-f^{\ell-1}(s)\right\|_{L^{1+\delta}\left(\Omega \times \mathbb{R}^{3}\right)}^{1+\delta} .
\end{aligned}
$$

Inductively we derive stability

$$
\begin{aligned}
& \quad \sup _{0 \leq s \leq t}\left\|f^{\ell}(s)-f^{m}(s)\right\|_{L^{1+\delta}\left(\Omega \times \mathbb{R}^{3}\right)}^{1+\delta}+\int_{0}^{t}\left|f^{\ell}(s)-f^{m}(s)\right|_{L^{1+\delta}(\gamma)} \\
& \leq O\left(t^{1+\delta}\right)^{\min \{\ell, m\}},
\end{aligned}
$$

and hence 6.51).

Step 6. We combine (6.11) and (6.51) to get unique weak-* convergence (up to subsequence if necessary), $\left(w_{\vartheta} f^{\ell}, w_{\vartheta} f^{\ell+1}\right) \stackrel{*}{\rightarrow}\left(w_{\vartheta} f, w_{\vartheta} f\right)$ weakly-* in $L^{\infty}\left(\mathbb{R} \times \Omega \times \mathbb{R}^{3}\right) \cap L^{\infty}(\mathbb{R} \times \gamma)$. For $\varphi \in C_{c}^{\infty}\left(\mathbb{R} \times \bar{\Omega} \times \mathbb{R}^{3}\right)$,

$$
\begin{aligned}
& \int_{0}^{T} \iint_{\Omega \times \mathbb{R}^{3}} f^{\ell+1}\left[-\partial_{t}-v \cdot \nabla_{x}+\nu\right] \varphi \\
& +\underbrace{f^{\ell+1}\left\{\nabla_{x} \phi^{\ell} \cdot \nabla_{v} \varphi+\frac{v}{2} \cdot \nabla_{x} \phi^{\ell} \varphi\right\}}_{[6.53]_{\phi}} \\
& =\int_{0}^{T} \iint_{\Omega \times \mathbb{R}^{3}} K f^{\ell} \varphi-v \cdot \nabla_{x} \phi^{\ell} \sqrt{\mu} \varphi \\
& +\underbrace{\Gamma_{\text {gain }}\left(f^{\ell}, f^{\ell}\right) \varphi}_{[6.53]_{\text {gain }}}-\underbrace{\Gamma_{\operatorname{loss}}\left(f^{\ell}, f^{\ell+1}\right) \varphi}_{[6.53]_{\text {loss }}} \\
& +\int_{0}^{T} \int_{\gamma_{+}} f^{\ell+1} \varphi-\int_{0}^{T} \int_{\gamma_{-}} c_{\mu} \sqrt{\mu} \int_{n \cdot u>0} f^{\ell} \sqrt{\mu}\{n \cdot u\} \mathrm{d} u \varphi .
\end{aligned}
$$

Except the underbraced terms in $(6.53)$ all terms converges to limits with $f$ instead of $f^{\ell+1}$ or $f^{\ell}$.

We define, for $(t, x, v) \in \mathbb{R} \times \bar{\Omega} \times \mathbb{R}^{3}$ and for $0<\delta \ll 1$,

$$
\begin{aligned}
f_{\delta}^{\ell}(t, x, v) & :=\kappa_{\delta}(x, v) f^{\ell}(t, x, v) \\
& :=\chi\left(\frac{|n(x) \cdot v|}{\delta}-1\right)[1-\chi(\delta|v|)] \chi\left(\frac{|v|}{\delta}-1\right) f^{\ell}(t, x, v) .
\end{aligned}
$$

Note that $f_{\delta}(t, x, v)=0$ if either $|n(x) \cdot v| \leq \delta,|v| \geq \frac{1}{\delta}$, or $|v| \leq \delta$.

From (6.11)

$$
\begin{aligned}
& \left|\int_{0}^{T} \iint_{(6.53)^{\text {loss }}}-\int_{0}^{T} \iint \Gamma_{\text {loss }}(f, f) \varphi\right| \\
\leq & \left|\int_{0}^{T} \iint_{\Omega \times \mathbb{R}^{3}} \int_{\mathbb{R}^{3}}\right| v-u\left|\left\{f^{\ell}(u)-f(u)\right\} \sqrt{\mu(u)} \mathrm{d} u f^{\ell+1}(v) \varphi(t, x, v) \mathrm{d} v \mathrm{~d} x \mathrm{~d} t\right| \\
+ & \left|\int_{0}^{T} \iint_{\Omega \times \mathbb{R}^{3}} \int_{\mathbb{R}^{3}}\right| v-u\left|f(u) \sqrt{\mu(u)} \mathrm{d} u\left\{f^{\ell+1}(v)-f(v)\right\} \varphi(t, x, v) \mathrm{d} v \mathrm{~d} x \mathrm{~d} t\right| .
\end{aligned}
$$


The second term converges to zero from the weak-* convergence in $L^{\infty}$ and (6.11). The first term is bounded by, from (6.11),

$$
\begin{aligned}
& {\left[\int_{0}^{T}\left\|\int_{\mathbb{R}^{3}} \kappa_{\delta}(x, u)\left(f^{\ell}(t, x, u)-f(t, x, u)\right)\langle u\rangle \sqrt{\mu(u)} \mathrm{d} u\right\|_{L^{2}\left(\Omega \times \mathbb{R}^{3}\right)}^{2}\right]^{1 / 2}} \\
& \times \sup _{0 \leq t \leq T}\left\|w_{\vartheta} f^{\ell+1}(t)\right\|_{\infty}+O(\delta) .
\end{aligned}
$$

On the other hand, from Lemma 14, we have an extension $\bar{f}^{\ell}(t, x, v)$ of $\kappa_{\delta}(x, u) f^{\ell}(t, x, u)$. We apply the average lemma (see Theorem 7.2.1 in page 187 of [20, for example) to $\bar{f}^{\ell}(t, x, v)$. From (6.14) and (6.11)

$$
\sup _{\ell}\left\|\int_{\mathbb{R}^{3}} \bar{f}^{\ell}(t, x, u)\langle u\rangle \sqrt{\mu(u)} \mathrm{d} u\right\|_{H_{t, x}^{1 / 4}\left(\mathbb{R} \times \mathbb{R}^{3}\right)}<\infty .
$$

Then by $H^{1 / 4} \subset \subset L^{2}$, up to subsequence, we conclude that

$$
\begin{aligned}
& \int_{\mathbb{R}^{3}} \kappa_{\delta}(x, u) f^{\ell}(t, x, u)\langle u\rangle \sqrt{\mu(u)} \mathrm{d} u \\
\rightarrow & \int_{\mathbb{R}^{3}} \kappa_{\delta}(x, u) f(t, x, u)\langle u\rangle \sqrt{\mu(u)} \mathrm{d} u \text { strongly in } L_{t, x}^{2} .
\end{aligned}
$$

So we conclude that 6.55 as $\ell \rightarrow \infty$.

For (6.53) gain let us use a test function $\varphi_{1}(v) \varphi_{2}(t, x)$. From the density argument, it suffices to prove a limit by testing with $\varphi(t, x, v)$.

We use a standard change of variables $(v, u) \mapsto\left(v^{\prime}, u^{\prime}\right)$ and $(v, u) \mapsto\left(u^{\prime}, v^{\prime}\right)$ (for example see page 10 of [20]) to get

$$
\begin{aligned}
& \left.\int_{0}^{T} \iint \sqrt{(6.53)}\right)_{\text {gain }}-\int_{0}^{T} \iint \Gamma_{\text {gain }}(f, f) \varphi \\
= & \int_{0}^{T} \iint_{\text {gain }}\left(f^{\ell}-f, f^{\ell}\right) \varphi+\int_{0}^{T} \iint \Gamma_{\text {gain }}\left(f, f^{\ell}-f\right) \varphi \\
= & \int_{0}^{T} \iint_{\Omega \times \mathbb{R}^{3}} \\
& \times\left(\int_{\mathbb{R}^{3}} \int_{\mathbb{S}^{2}}\left(f^{\ell}(t, x, u)-f(t, x, u)\right) \sqrt{\mu\left(u^{\prime}\right)}|(v-u) \cdot \omega| \varphi_{1}\left(v^{\prime}\right) \mathrm{d} \omega \mathrm{d} u\right) \\
& \times f^{\ell}(t, x, v) \varphi_{2}(t, x) \mathrm{d} v \mathrm{~d} x \mathrm{~d} t \\
+ & \int_{0}^{T} \iint_{\Omega \times \mathbb{R}^{3}}\left(\int_{\mathbb{R}^{3}} \int_{\mathbb{S}^{2}}\right. \\
& \left.\times\left(f^{\ell}(t, x, u)-f(t, x, u)\right) \sqrt{\mu\left(v^{\prime}\right)}|(v-u) \cdot \omega| \varphi_{1}\left(u^{\prime}\right) \mathrm{d} \omega \mathrm{d} u\right) \\
& \times f(t, x, v) \varphi_{2}(t, x) \mathrm{d} v \mathrm{~d} x \mathrm{~d} t .
\end{aligned}
$$

For $N \gg 1$ we decompose the integration of (6.58) and (6.59) using

$$
\begin{aligned}
1= & \{1-\chi(|u|-N)\}\{1-\chi(|v|-N)\} \\
& +\chi(|u|-N)+\chi(|v|-N)-\chi(|u|-N) \chi(|v|-N) .
\end{aligned}
$$

Note that $\{1-\chi(|u|-N)\}\{1-\chi(|v|-N)\} \neq 0$ if $|v| \leq N+1$ and $|u| \leq N+1$, and if $\chi(|u|-N)+\chi(|v|-N)-$ $\chi(|u|-N) \chi(|v|-N) \neq 0$ then either $|v| \geq N$ or $|u| \geq N$. From (6.11), the second part of (6.58) and (6.59) from (6.60) are bounded by

$$
\begin{aligned}
& \int_{0}^{T} \iint_{\Omega \times \mathbb{R}^{3}} \int_{\mathbb{R}^{3}} \int_{\mathbb{S}^{2}}[\cdots] \times\{\chi(|u|-N)+\chi(|v|-N)-\chi(|u|-N) \chi(|v|-N)\} \\
\leq & \sup _{\ell}\left\|w_{\vartheta} f^{\ell}\right\|_{\infty}\left\|w_{\vartheta} f\right\|_{\infty} \times\left\{e^{-\frac{\vartheta}{2}|v|^{2}} e^{-\frac{\vartheta}{2}|u|^{2}}\right\}\left\{\mathbf{1}_{|v| \geq N}+\mathbf{1}_{|u| \geq N}\right\} \\
\leq & O\left(\frac{1}{N}\right) .
\end{aligned}
$$


Now we only need to consider the parts with $\{1-\chi(|u|-N)\}\{1-\chi(|v|-N)\}$. Then

$$
\begin{aligned}
=\int_{0}^{T} \iint_{\Omega \times \mathbb{R}^{3}} & \int_{\mathbb{R}^{3}}\left(f^{\ell}(t, x, u)-f(t, x, u)\right) \\
\times & \{1-\chi(|u|-N)\}\left(\int_{\mathbb{S}^{2}} \sqrt{\mu\left(u^{\prime}\right)}|(v-u) \cdot \omega| \varphi_{1}\left(v^{\prime}\right) \mathrm{d} \omega\right) \mathrm{d} u \\
\times & \{1-\chi(|v|-N)\} f^{\ell}(t, x, v) \varphi_{2}(t, x) \mathrm{d} v \mathrm{~d} x \mathrm{~d} t .
\end{aligned}
$$

Let us define

$$
\Phi_{v}(u):=\{1-\chi(|u|-N)\} \int_{\mathbb{S}^{2}} \sqrt{\mu\left(u^{\prime}\right)}|(v-u) \cdot \omega| \varphi_{1}\left(v^{\prime}\right) \mathrm{d} \omega \text { for }|v| \leq N+1 .
$$

For $0<\delta \ll 1$ we have $O\left(\frac{N^{3}}{\delta^{3}}\right)$ number of $v_{i} \in \mathbb{R}^{3}$ such that $\left\{v \in \mathbb{R}^{3}:|v| \leq N+1\right\} \subset \bigcup_{i=1}^{O\left(\frac{N^{3}}{\delta^{3}}\right)} B\left(v_{i}, \delta\right)$. Since (6.62) is smooth in $u$ and $v$ and compactly supported, for $0<\varepsilon \ll 1$ we can always choose $\delta>0$ such that

$$
\left|\Phi_{v}(u)-\Phi_{v_{i}}(u)\right|<\varepsilon \text { if } v \in B\left(v_{i}, \delta\right) .
$$

Now we replace $\Phi_{v}(u)$ in the second line of (6.61) by $\Phi_{v_{i}}(u)$ whenever $v \in B\left(v_{i}, \delta\right)$. Moreover we use $\kappa_{\delta^{-}}$cut off in (6.54). If $v$ is included in several balls then we choose the smallest $i$. From (6.63) and (6.11) the difference of (6.61) and the one with $\Phi_{v_{i}}(u)$ can be controlled and we conclude that

$$
\begin{aligned}
\text { (6.61) }=\{O(\varepsilon) & +O(\delta)\} \sup _{\ell}\left\|w_{\vartheta} f^{\ell}\right\|_{\infty}^{2} \\
+ & \int_{0}^{T} \int_{\Omega} \sum_{i} \int_{\mathbb{R}^{3}} \mathbf{1}_{v \in B\left(v_{i}, \delta\right)} \\
& \times \int_{\mathbb{R}^{3}} \kappa_{\delta}(x, u)\left(f^{\ell}(t, x, u)-f(t, x, u)\right) \Phi_{v_{i}}(u) \mathrm{d} u \\
& \times\{1-\chi(|v|-N)\} f^{\ell}(t, x, v) \varphi_{2}(t, x) \mathrm{d} v \mathrm{~d} x \mathrm{~d} t .
\end{aligned}
$$

From Lemma 14 and the average lemma

$$
\max _{1 \leq i \leq O\left(\frac{N^{3}}{\delta^{3}}\right)} \sup _{\ell}\left\|\int_{\mathbb{R}^{3}} \kappa_{\delta}(x, u) f^{\ell}(t, x, u) \Phi_{v_{i}}(u) \mathrm{d} u\right\|_{H_{t, x}^{1 / 4}\left(\mathbb{R} \times \mathbb{R}^{3}\right)}<\infty .
$$

For $i=1$ we extract a subsequence $\ell_{1} \subset \mathcal{I}_{1}$ such that

$$
\begin{aligned}
& \int_{\mathbb{R}^{3}} \kappa_{\delta}(x, u) f^{\ell_{1}}(t, x, u) \Phi_{v_{i}}(u) \mathrm{d} u \\
\rightarrow & \int_{\mathbb{R}^{3}} \kappa_{\delta}(x, u) f(t, x, u) \Phi_{v_{i}}(u) \mathrm{d} u \text { strongly in } L_{t, x}^{2} .
\end{aligned}
$$

Successively we extract subsequences $\mathcal{I}_{O\left(\frac{N^{3}}{\delta^{3}}\right)} \subset \cdots \subset \mathcal{I}_{2} \subset \mathcal{I}_{1}$. Now we use the last subsequence $\ell \in \mathcal{I}_{O\left(\frac{N^{3}}{\delta^{3}}\right)}$ and redefine $f^{\ell}$ with it. Clearly we have (6.66) for all $i$. Finally we bound the last term of (6.64) by

$$
\begin{aligned}
& C_{\varphi_{2}, N} \max _{i} \int_{0}^{T}\left\|\int_{\mathbb{R}^{3}} \kappa_{\delta}(x, u)\left(f^{\ell}(t, x, u)-f(t, x, u)\right) \Phi_{v_{i}}(u) \mathrm{d} u\right\|_{L_{t, x}^{2}} \\
& \times \sup _{\ell}\left\|w_{\vartheta} f^{\ell}\right\|_{\infty} \\
& \rightarrow 0 \text { as } \ell \rightarrow \infty .
\end{aligned}
$$

Together with (6.64) we prove 6.58) $\rightarrow 0$. Similarly we can prove 6.59) $\rightarrow 0$.

Now we consider 6.53 . From

$$
-\left(\Delta \phi^{\ell}-\Delta \phi\right)=\int \kappa_{\delta}\left(f^{\ell}-f\right) \sqrt{\mu}+\int\left(1-\kappa_{\delta}\right)\left(f^{\ell}-f\right) \sqrt{\mu}
$$

we have

$$
\left\|\nabla_{x} \phi^{\ell}-\nabla_{x} \phi\right\|_{L_{t, x}^{2}} \leq\left\|\int \kappa_{\delta}\left(f^{\ell}-f\right) \sqrt{\mu}\right\|_{L_{t, x}^{2}}+O(\delta) \sup _{\ell}\left\|w_{\vartheta} f^{\ell}\right\|_{\infty} .
$$


Then following the previous argument, we prove $\nabla_{x} \phi^{\ell} \rightarrow \nabla_{x} \phi$ strongly in $L_{t, x}^{2}$ as $\ell \rightarrow \infty$. Combining with $w_{\vartheta} f^{\ell} \stackrel{*}{\rightarrow} w_{\vartheta} f$ in $L^{\infty}$, we prove $\int_{0}^{T} \iint_{\Omega \times \mathbb{R}^{3}}(6.53) \phi$ converges to $\int_{0}^{T} \iint_{\Omega \times \mathbb{R}^{3}} f\left\{\nabla_{x} \phi \cdot \nabla_{v} \varphi+\frac{v}{2} \cdot \nabla_{x} \phi \varphi\right\}$. This proves the existence of a (weak) solution $f \in L^{\infty}$.

Step \%. We claim (6.3). By the weak lower-semicontinuity of $L^{p}$ we know that (if necessary we further extract a subsequence out of the subsequence of Step 6)

$$
w_{\tilde{\vartheta}} \alpha_{f^{\ell, \varepsilon}}^{\beta} \nabla_{x, v} f^{\ell+1} \rightarrow \mathcal{F},
$$

and

and

We need to prove that

$$
\sup _{0 \leq t \leq T^{* *}}\|\mathcal{F}(t)\|_{p}^{p} \leq \liminf \sup _{0 \leq t \leq T^{* *}}\left\|w_{\tilde{\vartheta}} \alpha_{f^{\ell}, \varepsilon}^{\beta} \nabla_{x, v} f^{\ell+1}(t)\right\|_{p}^{p},
$$

$$
\int_{0}^{T^{* *}}|\mathcal{F}|_{p,+}^{p} \leq \liminf \int_{0}^{T^{* *}}\left|w_{\tilde{\vartheta}} \alpha_{f^{\ell}, \varepsilon}^{\beta} \nabla_{x, v} f^{\ell+1}(t)\right|_{p,+}^{p}
$$

$$
\mathcal{F}=w_{\tilde{\vartheta}} \alpha_{f, \varepsilon}^{\beta} \nabla_{x, v} f \text { almost everywhere except } \gamma_{0} .
$$

We claim that, up to some subsequence, for any given smooth test function $\psi \in C_{c}^{\infty}\left(\bar{\Omega} \times \mathbb{R}^{3} \backslash \gamma_{0}\right)$

$$
\begin{aligned}
& \lim _{\ell \rightarrow \infty} \int_{0}^{t} \iint_{\Omega \times \mathbb{R}^{3}} w_{\tilde{\vartheta}} \alpha_{f^{\ell}, \varepsilon}^{\beta} \nabla_{x, v} f^{\ell+1} \psi \mathrm{d} x \mathrm{~d} v \\
= & \int_{0}^{t} \iint_{\Omega \times \mathbb{R}^{3}} w_{\tilde{\vartheta}} \alpha_{f, \varepsilon}^{\beta} \nabla_{x, v} f \psi \mathrm{d} x \mathrm{~d} v .
\end{aligned}
$$

We note that we need to extract a single subsequence, let say $\left\{\ell_{*}\right\} \subset\{\ell\}$, satisfying (6.69) for all test functions in $C_{c}^{\infty}\left(\bar{\Omega} \times \mathbb{R}^{3} \backslash \gamma_{0}\right)$. Of course the convergent rate needs not to be uniform and it could vary with test functions.

For each $N \in \mathbb{N}$ we define a set

$$
\begin{aligned}
\mathcal{S}_{N}:= & \left\{(x, v) \in \bar{\Omega} \times \mathbb{R}^{3}: \operatorname{dist}(x, \partial \Omega) \leq \frac{1}{N} \text { and }|n(x) \cdot v| \leq \frac{1}{N}\right\} \\
& \cup\{|v|>N\} .
\end{aligned}
$$

For a given test function we can always find $N \gg 1$ such that

$$
\operatorname{supp}(\psi) \subset\left(\mathcal{S}_{N}\right)^{c}:=\bar{\Omega} \times \mathbb{R}^{3} \backslash \mathcal{S}_{N} .
$$

We will exam (6.69) by the identity obtained from the integration by parts

$$
\begin{aligned}
& \int_{0}^{t} \iint_{\Omega \times \mathbb{R}^{3}} w_{\tilde{\vartheta}} \alpha_{f^{\ell}, \varepsilon}^{\beta} \nabla_{x, v} f^{\ell+1} \psi \mathrm{d} x \mathrm{~d} v \\
= & -\int_{0}^{t} \iint_{\Omega \times \mathbb{R}^{3}} \alpha_{f^{\ell, \varepsilon}}^{\beta} f^{\ell+1} \nabla_{x, v}\left(w_{\tilde{\vartheta}} \psi\right) \mathrm{d} x \mathrm{~d} v \\
& +\int_{0}^{t} \iint_{\gamma} n \alpha_{f^{\ell, \varepsilon}}^{\beta} f^{\ell+1}\left(w_{\tilde{\vartheta}} \psi\right) \\
& -\int_{0}^{t} \iint_{\Omega \times \mathbb{R}^{3}} \nabla_{x, v} \alpha_{f^{\ell}, \varepsilon}^{\beta} f^{\ell+1}\left(w_{\tilde{\vartheta}} \psi\right) \mathrm{d} x \mathrm{~d} v .
\end{aligned}
$$

We finish this step by proving the convergence of (6.72) and (6.73). From (1.25) and (6.11), if $(x, v) \in\left(\mathcal{S}_{N}\right)^{c}$ then

$$
\begin{aligned}
& \sup _{\ell \geq 0}\left|\alpha_{f^{\ell}, \varepsilon}^{\beta}(t, x, v)\right| \\
& \lesssim|v|^{\beta}+(t+\varepsilon)^{\beta} \sup _{\ell \geq 0}\left\|\nabla \phi_{f^{\ell}}\right\|_{\infty}^{\beta} \\
& \lesssim N^{\beta}+\left(T^{* *}+\varepsilon\right)^{\beta} \sup _{\ell \geq 0}\left\|w_{\vartheta} f^{\ell}\right\|_{\infty}^{\beta} \\
& \leq C_{N}<+\infty .
\end{aligned}
$$

Hence we extract a subsequence (let say $\left.\left\{\ell_{N}\right\}\right)$ out of subsequence in Step 6 such that $\alpha_{f^{\ell_{N}, \varepsilon}}^{\beta} \stackrel{*}{\rightarrow} A \in L^{\infty}$ weakly* in $L^{\infty}\left(\left(0, T^{* *}\right) \times\left(\mathcal{S}_{N}\right)^{c}\right) \cap L^{\infty}\left(\left(0, T^{* *}\right) \times\left(\gamma \cap\left(\mathcal{S}_{N}\right)^{c}\right)\right)$. Note that $\alpha_{f^{\ell_{N}, \varepsilon}}^{\beta}$ satisfies $\left[\partial_{t}+v \cdot \nabla_{x}-\nabla_{x} \phi^{\ell_{N}} \cdot \nabla_{v}\right] \alpha_{f \ell_{N}, \varepsilon}^{\beta}=0$ and $\left.\alpha_{f^{\ell_{N}, \varepsilon}}^{\beta}\right|_{\gamma_{-}}=|n \cdot v|^{\beta}$. By passing a limit in the weak formulation we conclude that $\left[\partial_{t}+v \cdot \nabla_{x}-\nabla_{x} \phi_{f} \cdot \nabla_{v}\right] A=0$ 
and $\left.A\right|_{\gamma_{-}}=|n \cdot v|^{\beta}$. By the uniqueness of the Vlasov equation $\left(\nabla \phi_{f} \in W^{1, p}\right.$ for any $\left.p<\infty\right)$ we derive $A=\alpha_{f, \varepsilon}^{\beta}$ almost everywhere and hence conclude that

$$
\begin{aligned}
& \alpha_{f^{\ell_{N}, \varepsilon}}^{\beta} \stackrel{*}{\rightarrow} \alpha_{f, \varepsilon}^{\beta} \text { weakly -* } \\
& \text { in } L^{\infty}\left(\left(0, T^{* *}\right) \times\left(\mathcal{S}_{N}\right)^{c}\right) \cap L^{\infty}\left(\left(0, T^{* *}\right) \times\left(\gamma \cap\left(\mathcal{S}_{N}\right)^{c}\right)\right) .
\end{aligned}
$$

Now the convergence of (6.72) and (6.73) is a direct consequence of strong convergence of (6.51) and the weak-* convergence of (6.75).

Step 8. We devote the entire Step 8 to prove the convergence of (6.74).

Step 8-a. Let us choose $(x, v) \in\left(\mathcal{S}_{N}\right)^{c}$. From (1.31)

$$
\text { If } t_{\mathbf{b}}^{f^{\ell}} \geq t+\varepsilon \text { then } \alpha_{f^{\ell}, \varepsilon}(t, x, v)=1 .
$$

From now we only consider that case

$$
t_{\mathbf{b}}^{f^{\ell}}(t, x, v) \leq \varepsilon+t
$$

If $|v| \geq 2\left(\varepsilon+T^{* *}\right) \sup _{\ell}\left\|\nabla \phi^{\ell}\right\|_{\infty}$ then

$$
\begin{aligned}
\left|V^{f^{\ell}}(s ; t, x, v)\right| & \geq|v|-\int_{s}^{t}\left\|\nabla \phi^{\ell}(\tau)\right\|_{\infty} \mathrm{d} \tau \\
& \geq\left(\varepsilon+T^{* *}\right) \sup _{\ell}\left\|\nabla \phi^{\ell}\right\|_{\infty} \quad \text { for all } \ell \text { and } s \in\left[-\varepsilon, T^{* *}\right] .
\end{aligned}
$$

Then following the argument to get (3.33), which are based on the estimates (3.35) and (3.36), we derive

$$
\begin{aligned}
& \left|\tilde{\alpha}\left(s, X^{f^{\ell}}(s ; t, x, v), V^{f^{\ell}}(s ; t, x, v)\right)\right| \\
\geq & \frac{1}{C_{\Omega}} \tilde{\alpha}(t, x, v) e^{-C_{\Omega} \frac{|t-s|}{\left(\varepsilon+T^{* *}\right) \sup _{\ell}\left\|\nabla \phi^{\ell}\right\|_{\infty}}} \\
\geq & \frac{e^{-\frac{C_{\Omega}}{\sup _{\ell}\left\|\nabla \phi^{\ell}\right\|_{\infty}}}}{C_{\Omega}} \times \frac{1}{N} \quad \text { for all } \ell \text { and } s \in\left[-\varepsilon, T^{* *}\right] .
\end{aligned}
$$

Especially at $s=t-t_{\mathbf{b}}^{f^{\ell}}(t, x, v)$, from (3.32),

$$
\left|n\left(x_{\mathbf{b}}^{f^{\ell}}\right) \cdot v_{\mathbf{b}}^{f^{\ell}}\right| \geq \frac{e^{-\frac{C_{\Omega}}{\sup \ell_{\ell}\left\|\phi^{\ell}\right\| \infty}}}{C_{\Omega}} \times \frac{1}{N} \quad \text { for all } \ell .
$$

Step 8-b. From now on we assume (6.77). From $|v| \leq 2\left(\varepsilon+T^{* *}\right) \sup _{\ell}\left\|\nabla \phi^{\ell}\right\|_{\infty}$ and (1.22),

$$
\left|V^{f^{\ell}}(s ; t, x, v)\right| \leq 3\left(\varepsilon+T^{* *}\right) \sup _{\ell}\left\|\nabla \phi^{\ell}\right\|_{\infty} \text { for } s \in\left[-\varepsilon, T^{* *}\right] .
$$

Let $\left(X_{n}^{f^{\ell}}, X_{\|}^{f^{\ell}}, V_{n}^{f^{\ell}}, V_{\|}^{f^{\ell}}\right)$ satisfy (2.13), (2.11), and (2.15) with $E=-\nabla \phi^{\ell}$.

Let us define

$$
\tau_{1}:=\sup \left\{\tau \geq 0: V_{n}^{f^{\ell}}(s ; t, x, v) \geq 0 \text { for all } s \in\left[t-t_{\mathbf{b}}^{f^{\ell}}(t, x, v), \tau\right]\right\} .
$$

Since $\left(X^{f^{\ell}}(s ; t, x, v), V^{f^{\ell}}(s ; t, x, v)\right)$ is $C^{1}$ (note that $\left.\nabla \phi^{\ell} \in C_{t, x}^{1}\right)$ in $s$ we have $V_{n}^{f^{\ell}}\left(\tau_{1} ; t, x, v\right)=0$.

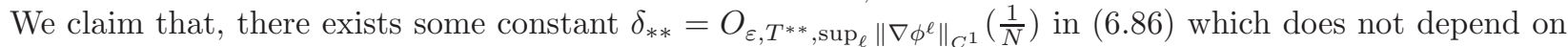
$\ell$ such that

$$
\begin{aligned}
& \text { If } 0 \leq V_{n}^{f^{\ell}}\left(t-t_{\mathbf{b}}^{f^{\ell}}(t, x, v) ; t, x, v\right)<\delta_{* *} \text { and } \underline{(6.79)}, \\
& \text { then } V_{n}^{f^{\ell}}(s ; t, x, v) \leq e^{C\left|s-\left(t-t_{\mathbf{b}}^{f^{\ell}}(t, x, v)\right)\right|^{2}} V_{n}^{f^{\ell}}\left(t-t_{\mathbf{b}}^{f^{\ell}}(t, x, v) ; t, x, v\right) \\
& \text { for } s \in\left[t-t_{\mathbf{b}}^{f^{\ell}}, \tau_{1}\right] .
\end{aligned}
$$

For the proof we regard the equations (2.13), (2.11), and (2.15) as the forward-in-time problem with an initial datum at $s=t-t_{\mathbf{b}}^{f^{\ell}}(t, x, v)$. Clearly we have $X_{n}^{f^{\ell}}\left(t-t_{\mathbf{b}}^{f^{\ell}}(t, x, v) ; t, x, v\right)=0$ and $V_{n}^{f^{\ell}}\left(t-t_{\mathbf{b}}^{f^{\ell}}(t, x, v) ; t, x, v\right) \geq 0$ from Lemma 2]. Again from Lemma 2] if $V_{n}^{f^{\ell}}\left(t-t_{\mathrm{b}}^{f^{\ell}}(t, x, v) ; t, x, v\right)=0$ then $X_{n}^{f^{\ell}}(s ; t, x, v)=0$ for all $s \geq$ $t-t_{\mathbf{b}}^{f^{\ell}}(t, x, v)$. From now on we assume $\left.V_{n}^{f^{\ell}}\left(t-t_{\mathbf{b}}^{f^{\ell}}(t, x, v) ; t, x, v\right)\right]>0$. From (2.15), as long as $t-t_{\mathbf{b}}^{f^{\ell}}(t, x, v) \leq$ $s \leq T^{* *}$ and

$$
V_{n}^{f^{\ell}}(s ; t, x, v) \geq 0 \text { and } X_{\perp}^{f^{\ell}}(s ; t, x, v) \leq \frac{1}{N} \ll 1,
$$


then we have

$$
\begin{aligned}
\dot{V}_{n}^{f^{\ell}}(s) & =\underbrace{\left[V_{\|}^{f^{\ell}}(s) \cdot \nabla^{2} \eta\left(X_{\|}^{f^{\ell}}(s)\right) \cdot V_{\|}^{f^{\ell}}(s)\right] \cdot n\left(X_{\|}^{f^{\ell}}(s)\right)}_{\leq 0 \text { from }[1.30]} \\
- & \underbrace{\nabla \phi^{\ell}\left(s, X^{f^{\ell}}(s)\right) \cdot\left[-n\left(X_{\|}^{f^{\ell}}(s)\right)\right]}_{=O(1)\left\{3\left(\varepsilon+T^{* *}\right) \sup _{\ell}\left\|\nabla \phi^{\ell}\right\|_{\infty}\right\}^{2} \times X_{n}^{f^{\ell}}(s) \text { from }[6.79} \\
& =\underbrace{O(1) \sup _{\ell}\left\|\nabla \phi^{\ell}\right\|_{C^{1}} \times X_{n}^{f^{\ell}}(s) \text { from }[2.18}_{\ell} \\
\leq & \left.C\left(1+\varepsilon+T^{* *}\right)^{2}\left(\sup _{\ell}^{f^{\ell}}(s)\left[V_{\|}^{f^{\ell}}(s) \cdot \nabla^{2}\left\|_{C^{1}} \sup _{\ell}\right\| \nabla \phi^{\ell} \|_{\infty}\right) \times X_{n}^{f^{\ell}}(s)\right) \cdot V_{\|}^{f^{\ell}}(s)\right] \cdot n\left(X_{\|}^{f^{\ell}}(s)\right)
\end{aligned}
$$

Let us consider (6.83) together with $\dot{X}_{n}^{f^{\ell}}(s ; t, x, v)=V_{n}^{f^{\ell}}(s ; t, x, v)$. Then, as long as satisfies (6.82),

$$
\begin{aligned}
& V_{n}^{f^{\ell}}(s)=V_{n}^{f^{\ell}}\left(t-t_{\mathbf{b}}^{f^{\ell}}\right)+\int_{t-t_{\mathbf{b}}^{f^{\ell}}}^{s} \dot{V}_{n}^{f^{\ell}}(\tau) \mathrm{d} \tau \\
& \leq V_{n}^{f^{\ell}}\left(t-t_{\mathbf{b}}^{f^{\ell}}\right) \\
& +\int_{t-t_{\mathrm{b}}^{f^{\ell}}}^{s} C\left(1+\varepsilon+T^{* *}\right)^{2}\left(\sup _{\ell}\left\|\nabla \phi^{\ell}\right\|_{C^{1}} \sup _{\ell}\left\|\nabla \phi^{\ell}\right\|_{\infty}\right) \times X_{n}^{f^{\ell}}(\tau) \mathrm{d} \tau \\
& =V_{n}^{f^{\ell}}\left(t-t_{\mathbf{b}}^{f^{\ell}}\right) \\
& +C\left(1+\varepsilon+T^{* *}\right)^{2}\left(\sup _{\ell}\left\|\nabla \phi^{\ell}\right\|_{C^{1}} \sup _{\ell}\left\|\nabla \phi^{\ell}\right\|_{\infty}\right) \\
& \times \int_{t-t_{\mathrm{b}}^{f \ell}}^{s} \int_{t-t_{\mathrm{b}}^{f \ell}}^{\tau} V_{n}^{f^{\ell}}\left(\tau^{\prime}\right) \mathrm{d} \tau^{\prime} \mathrm{d} \tau .
\end{aligned}
$$

Following the same argument of the proof of Lemma 10, we derive that

$$
\begin{aligned}
V_{n}^{f^{\ell}}(s) \leq & V_{n}^{f^{\ell}}\left(t-t_{\mathbf{b}}^{f^{\ell}}\right) \\
+ & C\left(1+\varepsilon+T^{* *}\right)^{2}\left(\sup _{\ell}\left\|\nabla \phi^{\ell}\right\|_{C^{1}} \sup _{\ell}\left\|\nabla \phi^{\ell}\right\|_{\infty}\right) \\
& \times \int_{t-t_{\mathbf{b}}^{f^{\ell}}}^{s}\left|s-\left(t-t_{\mathbf{b}}^{f^{\ell}}\right)\right| V_{n}^{f^{\ell}}\left(\tau^{\prime}\right) \mathrm{d} \tau^{\prime} .
\end{aligned}
$$

From the Gronwall's inequality, we derive that, as long as (6.82) holds,

$$
\begin{aligned}
& V_{n}^{f^{\ell}}(s ; t, x, v) \\
\leq & V_{n}^{f^{\ell}}\left(t-t_{\mathbf{b}}^{f^{\ell}}(t, x, v)\right) \\
& \times e^{C\left(1+\varepsilon+T^{* *}\right)^{2}\left(\sup _{\ell}\left\|\nabla \phi^{\ell}\right\|_{C^{1}} \sup _{\ell}\left\|\nabla \phi^{\ell}\right\|_{\infty}\right) \times\left|s-\left(t-t_{\mathbf{b}}^{\ell}(t, x, v)\right)\right|^{2} .}
\end{aligned}
$$

Now we verify the conditions of (6.82) for all $-\varepsilon \leq t-t_{\mathbf{b}}^{f^{\ell}}(t, x, v) \leq s \leq T^{* *}$. Note that we are only interested in the case of $V_{n}^{f^{\ell}}\left(t-t_{\mathbf{b}}^{f^{\ell}}(t, x, v) ; t, x, v\right)<\delta_{* *}$. From the argument of (6.83), ignoring negative curvature term,

$$
\begin{aligned}
& \left|X_{n}^{f^{\ell}}(s ; t, x, v)\right| \\
& \leq\left(\varepsilon+T^{* *}\right)\left|V_{n}^{f^{\ell}}\left(t_{\mathbf{b}}^{f^{\ell}} ; t, x, v\right)\right| \\
& +C\left[1+\left(\varepsilon+T^{* *}\right)^{2} \sup _{\ell}\left\|\nabla \phi^{\ell}\right\|_{\infty}\right] \sup _{\ell}\left\|\nabla \phi^{\ell}\right\|_{C^{1}} \\
& \quad \times \int_{t-t_{\mathbf{b}}^{f^{\ell}}}^{s} \int_{t-t_{\mathbf{b}}^{f^{\ell}}}^{\tau}\left|X_{n}^{f^{\ell}}(\tau ; t, x, v)\right| \mathrm{d} \tau \mathrm{d} s \\
& \leq\left(\varepsilon+T^{* *}\right)\left|V_{n}^{f^{\ell}}\left(t_{\mathbf{b}}^{f^{\ell}} ; t, x, v\right)\right| \\
& +C \int_{t-t_{\mathbf{b}}^{f^{\ell}}}^{s}\left|\tau-\left(t-t_{\mathbf{b}}^{f^{\ell}}\right)\right|\left|X_{n}^{f^{\ell}}(\tau ; t, x, v)\right| \mathrm{d} \tau . \\
& 58
\end{aligned}
$$


Then by the Gronwall's inequality we derive that, in case of (6.77),

$$
\begin{aligned}
& \left|X_{n}^{f^{\ell}}(s ; t, x, v)\right| \\
\leq & C_{\varepsilon+T^{* *}}\left|V_{n}^{f^{\ell}}\left(t-t_{\mathbf{b}}^{f^{\ell}} ; t, x, v\right)\right| \text { for all }-\varepsilon \leq t-t_{\mathbf{b}}^{f^{\ell}} \leq s \leq t \leq T^{* *} .
\end{aligned}
$$

If we choose

$$
\delta_{* *}=\frac{o(1)}{\left|T^{* *}+\varepsilon\right|} \times \frac{1}{N},
$$

then (6.84) holds for $-\varepsilon \leq t-t_{\mathrm{b}}^{f^{\ell}}(t, x, v) \leq s \leq T^{* *}$. Hence we complete the proof of (6.81).

Step 8-c. Suppose that (6.79) holds and $0 \leq V_{n}^{f^{\ell}}\left(t-t_{\mathbf{b}}^{f^{\ell}}(t, x, v) ; t, x, v\right)<\delta_{* *}$ with $\delta_{* *}$ of (6.86). Recall the definition of $\tau_{1}$ in (6.80). Inductively we define $\tau_{2}:=\sup \left\{\tau \geq 0: V_{n}^{f^{\ell}}(s ; t, x, v) \leq 0\right.$ for all $\left.s \in\left[\tau_{1}, \tau\right]\right\}$ and $\tau_{3}, \tau_{4}, \cdots$. Clearly such points can be countably many at most in an interval of $\left[t-t_{\mathbf{b}}^{f^{\ell}}, t\right]$. Suppose $\lim _{k \rightarrow \infty} \tau_{k}=t$. Then choose $k_{0} \gg 1$ such that $\left|\tau_{k_{0}}-t\right| \ll\left|V_{n}^{f^{\ell}}\left(t-t_{\mathrm{b}}^{f^{\ell}} ; t, x, v\right)\right|$. Then, for $s \in\left[\tau_{k_{0}}, t\right]$, from (6.83) and (6.79),

$$
\left|V_{n}^{f^{\ell}}(t ; t, x, v)\right| \lesssim\left|V_{n}^{f^{\ell}}\left(t-t_{\mathbf{b}}^{f^{\ell}} ; t, x, v\right)\right| .
$$

Now we assume that $\tau_{k_{0}}<t \leq \tau_{k_{0}+1}$. From the definition of $\tau_{i}$ in (6.80) we split the case in two.

Case 1: Suppose $V_{n}^{f^{\ell}}(s ; t, x, v)>0$ for $s \in\left(\tau_{k_{0}}, t\right)$.

From (6.83) and 6.85)

$$
V_{n}^{f^{\ell}}(t ; t, x, v) \lesssim \int_{\tau_{k_{0}}}^{T^{* *}} X_{n}^{f^{\ell}}(s) \lesssim\left|V_{n}^{f^{\ell}}\left(t-t_{\mathbf{b}}^{f^{\ell}} ; t, x, v\right)\right| .
$$

Case 2: Suppose $V_{n}^{f^{\ell}}(s ; t, x, v)<0$ for $s \in\left(\tau_{k_{0}}, t\right)$.

Suppose

$$
\begin{aligned}
-V_{n}^{f^{\ell}}(t ; t, x, v) & =\left|V_{n}^{f^{\ell}}(t ; t, x, v)\right| \\
& \geq\left|V_{n}^{f^{\ell}}\left(t-t_{\mathbf{b}}^{f^{\ell}} ; t, x, v\right)\right|^{A} \quad \text { for any } 0<A<\frac{1}{2} .
\end{aligned}
$$

From (6.83), now taking account of the curvature term this time, we derive that

$$
\begin{aligned}
-V_{n}^{f^{\ell}}(t ; t, x, v) \leq & \int_{\tau_{k_{0}}}^{t}(-1)\left[V_{\|}^{f^{\ell}}(s) \cdot \nabla^{2} \eta\left(X_{\|}^{f^{\ell}}(s)\right) \cdot V_{\|}^{f^{\ell}}(s)\right] \cdot n\left(X_{\|}^{f^{\ell}}(s)\right) \mathrm{d} s \\
& +C\left|V_{n}^{f^{\ell}}\left(t-t_{\mathbf{b}}^{f^{\ell}}(t, x, v) ; t, x, v\right)\right|,
\end{aligned}
$$

where we have used (6.79) and (6.85). From (6.89) the above inequality implies that, for $\left|V_{n}^{f^{\ell}}\left(t-t_{\mathbf{b}}^{f^{\ell}}(t, x, v) ; t, x, v\right)\right| \ll$ 1 ,

$$
\left|V_{n}^{f^{\ell}}\left(t-t_{\mathbf{b}}^{f^{\ell}} ; t, x, v\right)\right|^{A} \lesssim \int_{\tau_{k_{0}}}^{t}(-1)\left[V_{\|}^{f^{\ell}}(s) \cdot \nabla^{2} \eta\left(X_{\|}^{f^{\ell}}(s)\right) \cdot V_{\|}^{f^{\ell}}(s)\right] \cdot n\left(X_{\|}^{f^{\ell}}(s)\right) \mathrm{d} s .
$$

Note that $\left|\frac{d}{d s} V_{\|}^{f^{\ell}}(s)\right|$ and $\left|\frac{d}{d s} X_{\|}^{f^{\ell}}(s)\right|$ are all bound from $\nabla \phi^{\ell} \in C^{1}$, (6.79), and (6.85). Hence we have

$$
\begin{aligned}
& \frac{1}{2}\left|V_{n}^{f^{\ell}}\left(t-t_{\mathbf{b}}^{f^{\ell}} ; t, x, v\right)\right|^{A} \\
\lesssim & \int_{\tau_{k_{0}}}^{t-\left|V_{n}^{f^{\ell}}\left(t-t_{\mathrm{b}}^{f^{\ell}} ; t, x, v\right)\right|^{A}}(-1)\left[V_{\|}^{f^{\ell}}(s) \cdot \nabla^{2} \eta\left(X_{\|}^{f^{\ell}}(s)\right) \cdot V_{\|}^{f^{\ell}}(s)\right] \\
& \cdot n\left(X_{\|}^{f^{\ell}}(s)\right) \mathrm{d} s .
\end{aligned}
$$

On the other hand, if $t-\left|V_{n}^{f^{\ell}}\left(t-t_{\mathrm{b}}^{f^{\ell}} ; t, x, v\right)\right|^{A} \leq \tau_{k_{0}}$ then $\left|t-\tau_{k_{0}}\right| \leq\left|V_{n}^{f^{\ell}}\left(t-t_{\mathrm{b}}^{f^{\ell}} ; t, x, v\right)\right|^{A}$, which implies that, from 6.83), (6.79), and 6.85),

$$
\left|V_{n}^{f^{\ell}}(t ; t, x, v)\right| \lesssim\left|V_{n}^{f^{\ell}}\left(t-t_{\mathbf{b}}^{f^{\ell}} ; t, x, v\right)\right|^{A} \quad \text { for any } 0<A<\frac{1}{2} .
$$


Now we consider $X_{n}^{f^{\ell}}(t ; t, x, v)$. From (6.83) and $\dot{X}_{n}^{f^{\ell}}(s ; t, x, v)=V_{n}^{f^{\ell}}(s ; t, x, v)$ together with (6.85) and (6.79)

$$
\begin{aligned}
& X_{n}^{f^{\ell}}(t ; t, x, v) \\
\lesssim & \left|V_{n}^{f^{\ell}}\left(t-t_{\mathbf{b}}^{f^{\ell}} ; t, x, v\right)\right| \\
& +\int_{\tau_{k_{0}}}^{t} \int_{\tau_{k_{0}}}^{\tau} \underbrace{\left[V_{\|}^{f^{\ell}}(s) \cdot \nabla^{2} \eta\left(X_{\|}^{f^{\ell}}(s)\right) \cdot V_{\|}^{f^{\ell}}(s)\right] \cdot n\left(X_{\|}^{f^{\ell}}(s)\right)}_{\leq 0} \mathrm{~d} s \mathrm{~d} \tau \\
\lesssim & \left|V_{n}^{f^{\ell}}\left(t-t_{\mathbf{b}}^{f^{\ell}} ; t, x, v\right)\right| \\
& +\left|V_{n}^{f^{\ell}}\left(t-t_{\mathbf{b}}^{f^{\ell}} ; t, x, v\right)\right|^{A} \\
\times & \int_{\tau_{k_{0}}}^{t-\left|V_{n}^{f^{\ell}}\left(t-t_{\mathbf{b}}^{f^{\ell}} ; t, x, v\right)\right|^{A}}\left[V_{\|}^{f^{\ell}}(s) \cdot \nabla^{2} \eta\left(X_{\|}^{f^{\ell}}(s)\right) \cdot V_{\|}^{f^{\ell}}(s)\right] \cdot n\left(X_{\|}^{f^{\ell}}(s)\right) \mathrm{d} s \\
\lesssim & \left|V_{n}^{f^{\ell}}\left(t-t_{\mathbf{b}}^{f^{\ell}} ; t, x, v\right)\right|-\left|V_{n}^{f^{\ell}}\left(t-t_{\mathbf{b}}^{f^{\ell}} ; t, x, v\right)\right|^{2 A} \quad f r o m \underline{(6.90)} \\
\lesssim & \left|V_{n}^{f^{\ell}}\left(t-t_{\mathbf{b}}^{f^{\ell}} ; t, x, v\right)\right|-\left|V_{n}^{f^{\ell}}\left(t-t_{\mathbf{b}}^{f^{\ell}} ; t, x, v\right)\right|^{1-} \\
< & 0,
\end{aligned}
$$

for $\left|V_{n}^{f^{\ell}}\left(t-t_{\mathrm{b}}^{f^{\ell}} ; t, x, v\right)\right| \ll 1$. Clearly this cannot happen since $x \in \bar{\Omega}$ and $x_{n} \geq 0$. Therefore our assumption (6.89) was wrong and we conclude (6.91).

Step 8-d. From (6.81), 6.87), (6.88), and (6.91) in Step 8- $a$ and Step 8-b, we conclude that the same estimate (6.91) for $\left|V_{n}^{f^{\ell}}\left(t-t_{\mathrm{b}}^{f^{\ell}} ; t, x, v\right)\right| \ll 1$ in the case of (6.77) and (6.79). Finally from (6.76), (6.78), (6.81), and (6.91) Therefore we conclude that

$$
\left|V_{n}^{f^{\ell}}\left(t-t_{\mathbf{b}}^{f^{\ell}}(t, x, v) ; t, x, v\right)\right| \geq\left(\frac{1}{N}\right)^{1 / A}(t, x, v) \in\left[0, T^{* *}\right] \times\left(\mathcal{S}_{N}\right)^{c} .
$$

From (2.36), (2.37), (2.40), and (2.41) in Lemma 2.4 in [27],

$$
\begin{aligned}
& \sup _{\substack{\ell \in \mathbb{N},(x, v) \in\left(\mathcal{S}_{N}\right)^{c},-\varepsilon \leq t-t_{\mathbf{b}}^{f^{\ell}}(t, x, v) \leq t \leq T^{* *}}}\left|\nabla_{x, v} \alpha_{f^{\ell}, \varepsilon}^{\beta}(t, x, v)\right| \\
\lesssim & \frac{1}{\left|V_{n}^{f^{\ell}}\left(t-t_{\mathbf{b}}^{f^{\ell}} ; t, x, v\right)\right|^{2-\beta}} \lesssim_{\varepsilon, N, T^{* *}} 1 .
\end{aligned}
$$

Hence we extract another subsequence out of all previous steps (and redefine this as $\left\{\ell_{N}\right\}$ ) such that

$$
\nabla_{x, v} \alpha_{f^{\ell_{N}, \varepsilon}}^{\beta} \stackrel{*}{\rightarrow} \nabla_{x, v} \alpha_{f, \varepsilon}^{\beta} \text { weakly }-* \text { in } L^{\infty}\left(\left(-\varepsilon, T^{* *}\right) \times\left(\mathcal{S}_{N}\right)^{c}\right) .
$$

Note that the limiting function is identified from (6.75). Clearly the convergence of (6.74) is an easy consequence of strong convergence of (6.51) and the weak $-*$ convergence of (6.94).

Now we extract the final subsequence $\left\{\ell_{*}\right\}$ from the previous subsequence: By the Cantor's diagonal argument we define

$$
\ell_{*}=\ell_{\ell}
$$

Then clearly (6.69) holds with this subsequence for any test function $\psi$. For any $\psi \in C_{c}^{\infty}\left(\bar{\Omega} \times \mathbb{R}^{3} \backslash \gamma_{0}\right)$ there exists $N_{\psi} \in \mathbb{N}$ such that $\operatorname{supp}(\psi) \subset\left(\mathcal{S}_{N_{\psi}}\right)^{c}$. Then all the proofs work.

This implies (6.68) from (6.72), (6.73), (6.74). Positivity $F=\mu+\sqrt{\mu} f \geq 0$ comes from Step 1 and Step 6 .

Step 9. Choose $t>t^{\prime} \geq 0$. Instead of expanding $h(t, x, v)$ at $t=0$ as (6.24), we expand at $t^{\prime}$. Then by the iteration we have (6.28) replacing 0 by $t^{\prime}$. Collecting all terms at time $t^{\prime}$, we have

$$
\begin{aligned}
& \|h(t)\|_{\infty} \\
& \leq\left\|h\left(t^{\prime}\right)\right\|_{\infty}\left\{\mathbf{1}_{t_{1} \leq t^{\prime}} e^{-\int_{t^{\prime}}^{t} \nu}\right. \\
& \left.\quad+\mathbf{1}_{t_{1} \geq t^{\prime}} \frac{e^{-\int_{t_{1}}^{t} \nu}}{\widetilde{w}_{\vartheta}\left(V\left(t_{1}\right)\right)} \int_{\prod_{j=1}^{k-1} \mathcal{V}_{j}} \sum_{l=1}^{k-1} \mathbf{1}_{\left\{t_{l+1}^{\ell-l} \leq t^{\prime}<t_{l}^{\ell-(l-1)}\right\}} \mathrm{d} \Sigma_{l}\left(t^{\prime}\right)\right\} .
\end{aligned}
$$


Since (6.26) is a probability measure and $\left|e^{-\int_{t^{\prime}}^{t} \nu}-1\right| \ll\left|t-t^{\prime}\right|$ for $\left|t-t^{\prime}\right| \ll 1$,

$$
\left|\underline{(6.96)}-\left\|h\left(t^{\prime}\right)\right\|_{\infty}\right| \leq O\left(\left|t-t^{\prime}\right|\right)+\int_{\prod_{j=1}^{k-1} \mathcal{V}_{j}} \mathbf{1}_{\left\{t^{k}\left(t, x, v, u^{1}, \cdots, u^{k-1}\right)>0\right\}} \mathrm{d} \Sigma_{k-1}^{k-1} .
$$

Then by (6.28) we have $\|h(t)\|_{\infty}-\left\|h\left(t^{\prime}\right)\right\|_{\infty}<\frac{1}{2^{k}}+O_{k}\left(\left|t-t^{\prime}\right|\right)$. For large $k$, choosing $\left|t-t^{\prime}\right| \ll 1$, we can prove $\|h(t)\|_{\infty}-\left\|h\left(t^{\prime}\right)\right\|_{\infty} \ll 1$ as $\left|t-t^{\prime}\right| \ll 1$.

Now we can expand $h\left(t^{\prime}, x, v\right)$ at $t$ by (6.24). Following the same argument we have $\left\|h\left(t^{\prime}\right)\right\|_{\infty}-\|h(t)\|_{\infty} \ll 1$ as $\left|t-t^{\prime}\right| \ll 1$. Hence $\left\|w_{\vartheta} f(t)\right\|_{\infty}$ is continuous in $t$.

The continuity of $\left\|\nabla_{v} f(t)\right\|_{L_{x}^{3} L_{v}^{1+\delta}}$ and $\left\|w_{\tilde{\vartheta}} \alpha_{f, \varepsilon}^{\beta} \nabla_{x, v} f(t)\right\|_{p}^{p}+\int_{0}^{t}\left|w_{\tilde{\vartheta}} \alpha_{f, \varepsilon}^{\beta} \nabla_{x, v} f(t)\right|_{p,+}^{p}$ is an easy consequence of (5.11)-(5.16), and (4.74) as well.

\section{7. $L^{2}$ COERCIVITY}

The main purpose of this section is to prove the following:

Proposition 8. Suppose $(f, \phi)$ solves (1.15), (1.16), and (1.17). Then there is $0<\lambda_{2} \ll 1$ such that for $0 \leq s \leq t$,

$$
\begin{aligned}
& \left\|e^{\lambda_{2} t} f(t)\right\|_{2}^{2}+\left\|e^{\lambda_{2} t} \nabla \phi(t)\right\|_{2}^{2} \\
& +\int_{s}^{t}\left\|e^{\lambda_{2} \tau} f(\tau)\right\|_{\nu}^{2}+\left\|e^{\lambda_{2} \tau} \nabla \phi_{f}(\tau)\right\|_{2}^{2} \mathrm{~d} \tau+\int_{s}^{t}\left|e^{\lambda_{2} \tau} f\right|_{2,+}^{2} \\
\lesssim & \left\|e^{\lambda_{2} s} f(s)\right\|_{2}^{2}+\left\|e^{\lambda_{2} s} \nabla \phi_{f}(s)\right\|_{2}^{2} \\
& +\sup _{s \leq \tau \leq t}\left\|w_{\vartheta} f(\tau)\right\|_{\infty} \int_{s}^{t}\left\|e^{\lambda_{2} \tau} f(\tau)\right\|_{\nu}^{2} .
\end{aligned}
$$

The null space of linear operator $L$ is a five-dimensional subspace of $L_{v}^{2}\left(\mathbb{R}^{3}\right)$ spanned by orthonormal vectors $\left\{\sqrt{\mu}, v \sqrt{\mu}, \frac{|v|^{2}-3}{2} \sqrt{\mu}\right\}$ and the projection of $f$ onto the null space $N(L)$ is denoted by

$$
\mathbf{P} f(t, x, v):=\left\{a(t, x)+v \cdot b(t, x)+\frac{|v|^{2}-3}{2} c(t, x)\right\} \sqrt{\mu} .
$$

In order to prove the proposition we need the following:

Lemma 13. There exists a function $G(t)$ such that, for all $0 \leq s \leq t, G(s) \lesssim\|f(s)\|_{2}^{2}$ and

$$
\begin{aligned}
& \int_{s}^{t}\|\mathbf{P} f(\tau)\|_{\nu}^{2}+\int_{s}^{t}\left\|\nabla \phi_{f}\right\|_{2}^{2} \\
\lesssim & G(t)-G(s)+\int_{s}^{t}\|(\mathbf{I}-\mathbf{P}) f(\tau)\|_{\nu}^{2}+\int_{s}^{t}\left|\left(1-P_{\gamma}\right) f(\tau)\right|_{2,+}^{2} \\
& +\int_{s}^{t}\left\|\nu^{-1 / 2} \Gamma(f, f)\right\|_{2}^{2}+\int_{s}^{t}\left\|w_{\vartheta} f(\tau)\right\|_{\infty}^{2}\|\mathbf{P} f(\tau)\|_{2}^{2} .
\end{aligned}
$$

Proof of Proposition 8. Step 1. Without loss of generality we prove the result with $s=0$. We have an $L^{2}$-estimate from $e^{\lambda_{2} t} \times(1.15)$

$$
\begin{aligned}
& \left\|e^{\lambda_{2} t} f(t)\right\|_{2}^{2}-\|f(0)\|_{2}^{2}+\int_{0}^{t}\left|e^{\lambda_{2} \tau}\left(1-P_{\gamma}\right) f^{j}\right|_{2,+}^{2} \\
& +\int_{0}^{t} \iint_{\Omega \times \mathbb{R}^{3}} v \cdot \nabla \phi_{f} e^{2 \lambda_{2} \tau}|f|^{2}+2 \int_{0}^{t} \iint_{\Omega \times \mathbb{R}^{3}} e^{\lambda_{2} \tau} 2 f L f \\
& =2 \int_{0}^{t} \iint_{\Omega \times \mathbb{R}^{3}} e^{2 \lambda_{2} \tau} f \Gamma(f, f)-2 \int_{0}^{t} e^{2 \lambda_{2} \tau} \int_{\Omega} \nabla \phi_{f} \cdot \int_{\mathbb{R}^{3}} v \sqrt{\mu} f \\
& +2 \lambda_{2} \int_{0}^{t}\left\|e^{\lambda_{2} \tau} f(\tau)\right\|_{2}^{2}
\end{aligned}
$$

where

$$
P_{\gamma} f:=c_{\mu} \sqrt{\mu(v)} \int_{n(x) \cdot u>0} f(u) \sqrt{\mu(u)}\{n(x) \cdot u\} \mathrm{d} u .
$$


On the other hand multiplying $\sqrt{\mu(v)} \phi_{f}(t, x)$ with a test function $\psi(t, x)$ to (1.15) and applying the Green's identity, we obtain

$$
\begin{aligned}
& \int_{\Omega} \nabla \phi_{f}(t, x) \cdot \int_{\mathbb{R}^{3}} v \sqrt{\mu} f \mathrm{~d} v \mathrm{~d} x \\
= & \int_{\Omega} \phi_{f}(t, x) \partial_{\tau}\left(\int_{\mathbb{R}^{3}} f(\tau) \sqrt{\mu} \mathrm{d} v\right) \mathrm{d} x+\iint_{\partial \Omega \times \mathbb{R}^{3}} \phi_{f}(t, x) f \sqrt{\mu}\{n \cdot v\} \mathrm{d} v \mathrm{~d} S_{x} .
\end{aligned}
$$

From (1.7), the last boundary contribution equals zero. Now we use (1.16) and deduce that

$$
\begin{aligned}
& \int_{0}^{t} e^{2 \lambda_{2} \tau} \int_{\Omega} \phi_{f}(t, x) \partial_{\tau}\left(\int_{\mathbb{R}^{3}} f(\tau) \sqrt{\mu} \mathrm{d} v\right) \mathrm{d} x \mathrm{~d} \tau \\
= & -\int_{0}^{t} e^{2 \lambda_{2} \tau} \int_{\Omega} \phi_{f}(t, x) \partial_{\tau} \Delta_{x} \phi_{f}(\tau, x) \mathrm{d} x \mathrm{~d} \tau \\
= & \frac{1}{2} \int_{0}^{t} e^{2 \lambda_{2} \tau} \int_{\Omega} \partial_{\tau}\left|\nabla_{x} \phi_{f}(\tau, x)\right|^{2} \mathrm{~d} x \mathrm{~d} \tau \\
= & \frac{1}{2}\left(\int_{\Omega} e^{2 \lambda_{2} t}\left|\nabla_{x} \phi_{f}(t, x)\right|^{2} \mathrm{~d} x\right)-\frac{1}{2}\left(\int_{\Omega}\left|\nabla_{x} \phi_{f}(0, x)\right|^{2} \mathrm{~d} x\right) \\
& -\lambda_{2} \int_{0}^{t} e^{2 \lambda_{2} \tau} \int_{\Omega}\left|\nabla_{x} \phi_{f}(\tau, x)\right|^{2} \mathrm{~d} x \mathrm{~d} \tau .
\end{aligned}
$$

Hence we derive

$$
\begin{aligned}
& \left\|e^{\lambda_{2} t} f(t)\right\|_{2}^{2}+\left\|e^{\lambda_{2} t} \nabla \phi_{f}(t)\right\|_{2}^{2}+\int_{0}^{t} \iint_{\Omega \times \mathbb{R}^{3}} e^{2 \lambda_{2} \tau} v \cdot \nabla \phi_{f}|f|^{2} \\
& +2 C \int_{0}^{t} \iint_{\Omega \times \mathbb{R}^{3}}\left\|e^{\lambda_{2} \tau}(\mathbf{I}-\mathbf{P}) f\right\|_{\nu}^{2}+\int_{0}^{t}\left|e^{\lambda_{2} \tau}\left(1-P_{\gamma}\right) f^{j}\right|_{2,+}^{2} \\
& \lesssim\|f(0)\|_{2}^{2}+\left\|\phi_{f}(0)\right\|_{2}^{2}+\int_{0}^{t}\left\|e^{\lambda_{2} \tau} \nu^{-1 / 2} \Gamma(f, f)\right\|_{2}^{2} \\
& \quad+\left\{\lambda_{2}+o(1)\right\} \int_{0}^{t}\left\|e^{\lambda_{2} \tau} f\right\|_{\nu}^{2}+\lambda_{2} \int_{0}^{t}\left\|e^{\lambda_{2} \tau} \nabla_{x} \phi_{f}\right\|_{2}^{2} .
\end{aligned}
$$

Now we apply Lemma 13 and add $o(1) \times(7.3)$ to the above inequality and choose $0<\lambda_{2} \ll 1$ to conclude (7.1) except the full boundary control.

Step 2. Note that from (7.4), $P_{\gamma} f=z(t, x) \sqrt{\mu(v)}$ for a suitable function $z(t, x)$ on the boundary. Then for $0<\varepsilon \ll 1$

$$
\begin{aligned}
\left|P_{\gamma} f\right|_{\gamma, 2}^{2} & =\int_{\partial \Omega}|z(t, x)|^{2} \mathrm{~d} x \times \int_{\mathbb{R}^{3}} \mu(v)|n(x) \cdot v| \mathrm{d} v \\
& \lesssim \int_{\partial \Omega}|z(t, x)|^{2} \mathrm{~d} x \times \int_{\gamma_{+}(x) \backslash \gamma_{+}^{\varepsilon}(x)} \mu(v)^{3 / 2}|n(x) \cdot v| \mathrm{d} v \\
& =\left|\mathbf{1}_{\gamma_{+} \backslash \gamma_{+}^{\varepsilon}} \mu^{1 / 4} P_{\gamma} f\right|_{2,+}^{2} .
\end{aligned}
$$

Since $P_{\gamma} f=f-\left(1-P_{\gamma}\right) f$ on $\gamma_{+}$we have

$$
\left|\mathbf{1}_{\gamma_{+} \backslash \gamma_{+}^{\varepsilon}} \mu^{1 / 4} P_{\gamma} f\right|_{2,+}^{2} \lesssim\left|\mathbf{1}_{\gamma_{+} \backslash \gamma_{+}^{\varepsilon}} \mu^{1 / 4} f\right|_{2,+}^{2}+\left|\left(1-P_{\gamma}\right) f\right|_{2,+}^{2} .
$$

Therefore

$$
\int_{0}^{t}\left|P_{\gamma} f\right|_{\gamma, 2}^{2} \lesssim \int_{0}^{t}\left|\mathbf{1}_{\gamma_{+} \backslash \gamma_{+}^{\S}} \mu^{1 / 4} f\right|_{2,+}^{2}+\int_{0}^{t}\left|\left(1-P_{\gamma}\right) f\right|_{2,+}^{2}
$$

Note that

$$
\begin{aligned}
& \left|\left[\partial_{t}+v \cdot \nabla_{x}-\nabla \phi \cdot \nabla_{v}\right]\left(\mu^{1 / 4} f\right)\right| \\
\lesssim & \mu^{1 / 4}\left\{|v|\left|\nabla_{x} \phi\right| f+|v|\left|\nabla_{x} \phi\right|+|L f|+|\Gamma(f, f)|\right\} .
\end{aligned}
$$


By the trace theorem Lemma 7

$$
\begin{aligned}
& \int_{0}^{t}\left|\mathbf{1}_{\gamma_{+} \backslash \gamma_{+}^{\varepsilon}} \mu^{1 / 4} f\right|_{2,+}^{2} \\
\lesssim & \left\|f_{0}\right\|_{2}+\left(1+\|w f\|_{\infty}\right) \int_{0}^{t}\|f\|_{2}^{2}+\int_{0}^{t}\|\nabla \phi\|_{2}^{2} .
\end{aligned}
$$

Adding $o(1) \times(7.5)$ to the result of Step 1 and using (7.6) we conclude (7.1).

Proof of Lemma 13. From the Green's identity, a solution $\left(f, \phi_{f}\right)$ satisfies

$$
\begin{aligned}
& \iint_{\Omega \times \mathbb{R}^{3}} f(t) \psi(t)-\iint_{\Omega \times \mathbb{R}^{3}} f(s) \psi(s) \\
& -\underbrace{\int_{s}^{t} \iint_{\Omega \times \mathbb{R}^{3}} f \partial_{t} \psi}_{[7.7]_{T}}+\underbrace{\int_{s}^{t} \int_{\gamma} \psi f(v \cdot n(x))}_{\left[7.7 \mathrm{~T}_{B}\right.} \\
& -\underbrace{\int_{s}^{t} \iint_{\Omega \times \mathbb{R}^{3}} \mathbf{P} f v \cdot \nabla_{x} \psi}_{[7.7]_{C}}-\int_{s}^{t} \iint_{\Omega \times \mathbb{R}^{3}}(\mathbf{I}-\mathbf{P}) f v \cdot \nabla_{x} \psi \\
& +\underbrace{\int_{s}^{t} \iint_{\Omega \times \mathbb{R}^{3}} \sqrt{\mu} f \nabla_{x} \phi_{f} \cdot \nabla_{v}\left[\frac{1}{\sqrt{\mu}} \psi\right]}_{\text {7.7 } P_{P}} \\
& =\int_{s}^{t} \iint_{\Omega \times \mathbb{R}^{3}} \psi\{-L(\mathbf{I}-\mathbf{P}) f+\Gamma(f, f)\} \\
& -\underbrace{\int_{s}^{t} \iint_{\Omega \times \mathbb{R}^{3}} \psi v \cdot \nabla_{x} \phi_{f} \sqrt{\mu}}_{[7.7]_{\phi_{f}}} .
\end{aligned}
$$

As [9, 8, we use a set of test functions:

$$
\begin{aligned}
\psi_{a} & \equiv\left(|v|^{2}-\beta_{a}\right) \sqrt{\mu} v \cdot \nabla_{x} \varphi_{a}, \\
\psi_{b, 1}^{i, j} & \equiv\left(v_{i}^{2}-\beta_{b}\right) \sqrt{\mu} \partial_{j} \varphi_{b}^{j}, \quad i, j=1,2,3, \\
\psi_{b, 2}^{i, j} & \equiv|v|^{2} v_{i} v_{j} \sqrt{\mu} \partial_{j} \varphi_{b}^{i}(x), \quad i \neq j, \\
\psi_{c} & \equiv\left(|v|^{2}-\beta_{c}\right) \sqrt{\mu} v \cdot \nabla_{x} \varphi_{c},
\end{aligned}
$$

where $\varphi_{a}(t, x), \varphi_{b}(t, x)$, and $\varphi_{c}(t, x)$ solve

$$
\begin{aligned}
& -\Delta \varphi_{a}=a(t, x),\left.\quad \partial_{n} \varphi_{a}\right|_{\partial \Omega}=0, \\
& -\Delta \varphi_{b}^{j}=b_{j}(t, x),\left.\quad \varphi_{b}^{j}\right|_{\partial \Omega}=0, \text { and }-\Delta \varphi_{c}=c(t, x),\left.\quad \varphi_{c}\right|_{\partial \Omega}=0,
\end{aligned}
$$

and $\beta_{a}=10, \beta_{b}=1$, and $\beta_{c}=5$ such that for all $i=1,2,3$,

$$
\begin{aligned}
& \int_{\mathbb{R}^{3}}\left(|v|^{2}-\beta_{a}\right)\left(\frac{|v|^{2}}{2}-\frac{3}{2}\right) v_{i}^{2} \mu(v) \mathrm{d} v=0, \\
& \int_{\mathbb{R}^{3}}\left(v_{i}^{2}-\beta_{b}\right) \mu(v) \mathrm{d} v=0, \\
& \int_{\mathbb{R}^{3}}\left(|v|^{2}-\beta_{c}\right) v_{i}^{2} \mu(v) \mathrm{d} v=0 .
\end{aligned}
$$


Step 1. Estimate of (7.7) $\phi_{f}$ : From (7.8) and (17.10), we have (7.7) $\phi_{f} \equiv 0$ for $\psi_{b, 1}^{i, j}, \psi_{b, 2}^{i, j}$, and $\psi_{c}$. For $\psi=\psi_{a}$, (7.7) $\phi_{f}$ equals

$$
\begin{aligned}
\left.(17.7)_{\phi_{f}}\right|_{\psi=\psi_{a}} & =\int_{\mathbb{R}^{3}}\left(|v|^{2}-\beta_{a}\right)\left(v_{1}\right)^{2} \mu \mathrm{d} v \int_{s}^{t} \int_{\Omega} \nabla \varphi_{a} \cdot \nabla \phi_{f} \\
& =C \int_{s}^{t}\left\|\nabla \phi_{f}\right\|_{2}^{2}, \\
& \left.(17.7)_{\phi_{f}}\right|_{\psi=\psi_{b}, \psi_{c}} \\
= & 0,
\end{aligned}
$$

because $\varphi_{a}=\phi_{f}$ from the definitions of (1.16) and (7.9).

Estimate of (7.7) $P$ : From (7.8),

$$
\begin{aligned}
\left(\sqrt{7.7)_{P}} P\right. & =\int_{s}^{t} \iint_{\Omega \times \mathbb{R}^{3}} \sqrt{\mu} f \nabla_{x} \phi_{f} \cdot \nabla_{v}\left[\frac{1}{\sqrt{\mu}} \psi\right], \quad \psi=\psi_{a, b, c}, \\
& \lesssim \int_{s}^{t}\left\|w_{\vartheta} f\right\|_{\infty} \int_{\Omega} \nabla_{x} \phi_{f} \cdot \nabla_{x} \varphi_{a, b, c} \lesssim \int_{s}^{t}\left\|w_{\vartheta} f(\tau)\right\|_{\infty}\|\mathbf{P} f(\tau)\|_{2}^{2},
\end{aligned}
$$

by elliptic estimate $\left\|\nabla_{x} \varphi_{a, b, c}\right\|_{2} \lesssim\left\|\varphi_{a, b, c}\right\|_{H^{2}} \lesssim\|\mathbf{P} f\|_{2}$.

Step 2. Estimate of $c$ : We apply $\varphi_{c}$ to (7.7). From oddness in velocity integration and (7.10), (7.7) $C$ becomes,

$$
\int_{s}^{t} \iint_{\Omega \times \mathbb{R}^{3}} \mathbf{P} f v \cdot \nabla_{x} \psi_{c}=C_{1} \int_{s}^{t}\|c(\tau)\|_{2}^{2}
$$

where $\int_{\mathbf{R}^{3}}\left(|v|^{2}-\beta_{c}\right) v_{i}^{2}\left(\frac{|v|^{2}}{2}-\frac{3}{2}\right) \mu(v) d v=10 \pi \sqrt{2 \pi}$. For boundary integral (7.7) $B$, we decompose $f_{\gamma}=P_{\gamma} f+$ $\mathbf{1}_{\gamma_{+}}\left(1-P_{\gamma}\right) f$. Then from (17.10) and trace theorem $\left|\nabla \varphi_{c}\right|_{2} \lesssim\left\|\varphi_{c}\right\|_{H^{2}} \lesssim\|c\|_{2}$,

$$
\begin{aligned}
& \int_{s}^{t} \int_{\gamma} \psi_{c} f(v \cdot n(x))=\int_{s}^{t} \int_{\gamma} \psi_{c} \mathbf{1}_{\gamma_{+}}\left(1-P_{\gamma}\right) f d \gamma \\
& \lesssim \varepsilon \int_{s}^{t}\|c(\tau)\|_{2}^{2}+C_{\varepsilon} \int_{s}^{t}\left|\left(1-P_{\gamma}\right) f(\tau)\right|_{2,+}^{2}, \quad \varepsilon \ll 1 .
\end{aligned}
$$

If we define

$$
\begin{aligned}
R e:= & (\sqrt{7.7})_{P}+(\mathbf{7 . 7})_{\phi_{f}}+\int_{s}^{t} \iint_{\Omega \times \mathbb{R}^{3}} \psi\{L(\mathbf{I}-\mathbf{P}) f-\Gamma(f, f)\} \\
& -\int_{s}^{t} \iint_{\Omega \times \mathbb{R}^{3}}(\mathbf{I}-\mathbf{P}) f v \cdot \nabla_{x} \psi,
\end{aligned}
$$

then

$$
\begin{aligned}
& \left.\int_{s}^{t} \iint_{\Omega \times \mathbb{R}^{3}} \psi_{c} R e\right|_{\psi_{c}} \\
\lesssim & \varepsilon \int_{s}^{t}\|c\|_{2}^{2}+\int_{s}^{t}\|(\mathbf{I}-\mathbf{P}) f(\tau)\|_{\nu}^{2}+\int_{s}^{t}\left\|\nu^{-1 / 2} \Gamma(f, f)(\tau)\right\|_{2}^{2},
\end{aligned}
$$

from elliptic estimate and Young's inequality. We also use even/oddness in velocity integration, (7.10), and Young's inequality to estimate,

$$
\begin{aligned}
\left(\text { (7.7) }\left._{T}\right|_{\psi_{c}}\right. & =\int_{s}^{t} \iint_{\Omega \times \mathbb{R}^{3}} f \partial_{t} \psi_{c}=\int_{s}^{t} \iint_{\Omega \times \mathbb{R}^{3}}(\mathbf{I}-\mathbf{P}) f \partial_{t} \psi_{c} \\
& \lesssim \varepsilon \int_{s}^{t}\left\|\nabla \Delta^{-1} \partial_{t} c(\tau)\right\|_{2}^{2}+\int_{s}^{t}\|(\mathbf{I}-\mathbf{P}) f(\tau)\|_{\nu}^{2}
\end{aligned}
$$

Now, we choose a new test function $\psi_{c}^{t}:=\left(\frac{|v|^{2}}{2}-\frac{3}{2}\right) \sqrt{\mu} \partial_{t} \varphi_{c}(t, x)$. Note that $\partial_{t} \varphi_{c}$ solves $-\Delta \partial_{t} \varphi_{c}=\partial_{t} c(t, x)$ with $\left.\partial_{t} \varphi_{c}(t, x)\right|_{\partial \Omega}=0$. We taking difference quotient for $\partial_{t} f$ in (1.15) and it replace first three terms in the 
LHS of (17.7). With help of Poincaré inequality $\left\|\partial_{t} \varphi_{c}\right\|_{2} \lesssim\left\|\nabla \partial_{t} \varphi_{c}\right\|_{2}$, we can also compute

$$
\begin{aligned}
\left.(17.7)_{\phi_{f}}\right|_{\psi=\psi_{c}^{t}} & =\int_{s}^{t} \iint_{\Omega \times \mathbb{R}^{3}}\left(\frac{|v|^{2}}{2}-\frac{3}{2}\right) \sqrt{\mu} \partial_{t} \varphi_{c} v \cdot \nabla_{x} \phi_{f} \sqrt{\mu} \\
& \lesssim \varepsilon \int_{s}^{t}\left\|\nabla \Delta^{-1} \partial_{t} c(\tau)\right\|_{2}^{2}+\int_{s}^{t}\|a(\tau)\|_{2}^{2}, \\
\left.(17.7)_{P}\right|_{\psi=\psi_{c}^{t}} & =\int_{s}^{t} \iint_{\Omega \times \mathbb{R}^{3}} \sqrt{\mu} f \nabla_{x} \phi_{f} \cdot 2 v \partial_{t} \varphi_{c} \\
\lesssim & \int_{s}^{t}\left\|\nabla \Delta^{-1} \partial_{t} c(\tau)\right\|_{2}^{2}+\int_{s}^{t}\left(\|a(\tau)\|_{2}^{2}+\|b(\tau)\|_{2}^{2}\right) \\
& +\int_{s}^{t}\|(\mathbf{I}-\mathbf{P}) f\|_{\nu}^{2} .
\end{aligned}
$$

Since $\psi_{c}^{t}$ vanishes when it acts with $L f$ and $\Gamma(f, f)$, and boundary integral (7.7) $B$ vanishes by Dirichlet boundary condition of $\varphi_{c}$, from (7.18) and (7.7), we obtain

$$
\begin{aligned}
& \int_{s}^{t} \int_{\Omega} \partial_{t} \varphi_{c}(\tau, x) \partial_{t} c(\tau, x) d x=\int_{s}^{t}\left\|\nabla \Delta^{-1} \partial_{t} c(\tau)\right\|_{2}^{2} \\
& \lesssim \varepsilon \int_{s}^{t}\left\|\nabla \Delta^{-1} \partial_{t} c(\tau)\right\|_{2}^{2}+\int_{s}^{t}\left(\|a(\tau)\|_{2}^{2}+\|b(\tau)\|_{2}^{2}\right) \\
& \quad+\int_{s}^{t}\|(\mathbf{I}-\mathbf{P}) f(\tau)\|_{\nu}^{2} .
\end{aligned}
$$

We combine (7.7), (7.11), (7.12), (7.14), (7.16), (7.17), and (7.19) with $\varepsilon \ll 1$ to obtain

$$
\begin{aligned}
& \int_{s}^{t}\|c(\tau)\|_{2}^{2} \\
& \lesssim G_{c}(t)-G_{c}(s)+\int_{s}^{t}\|(\mathbf{I}-\mathbf{P}) f(\tau)\|_{\nu}^{2}+\int_{s}^{t}\left|\left(1-P_{\gamma}\right) f(\tau)\right|_{2,+}^{2} \\
& \quad+\int_{s}^{t}\left\|\nu^{-1 / 2} \Gamma(f, f)(\tau)\right\|_{2}^{2}+\int_{s}^{t}\left\|w_{\vartheta} f(\tau)\right\|_{\infty}^{2}\|\mathbf{P} f(\tau)\|_{2}^{2} \\
& \quad+\varepsilon \int_{s}^{t}\left(\|a(\tau)\|_{2}^{2}+\|b(\tau)\|_{2}^{2}\right),
\end{aligned}
$$

for $\varepsilon \ll 1$ where $G_{c}(t):=\iint_{\Omega \times \mathbb{R}^{3}} f(t) \psi_{c}(t) \lesssim\|f(t)\|_{2}^{2}$.

Step 3. Estimate of $a$ : From mass conservation $\int_{\Omega} a(t, x) d v=0, \varphi_{a}$ in (7.9) is well-defined. Moreover, we choose $\varphi_{a}$ so that has mean zero, $\int_{\Omega} \varphi_{a}(t, x) d x=0$. Therefore, Poincaré inequality $\left\|\varphi_{a}\right\|_{2} \lesssim\left\|\nabla \varphi_{a}\right\|_{2}$ holds and these are also true for $\partial_{t} \varphi_{a}$ which solves same elliptic equation with Neumann boundary condition.

By even/oddness in velocity integral and $\beta_{a}$ defined in (7.10), we can replace $c$ into $a$ in estimates (7.13) and (7.16). For boundary integral (7.7) ${ }_{B}$, we decompose $f_{\gamma}=P_{\gamma} f+\mathbf{1}_{\gamma_{+}}\left(1-P_{\gamma}\right) f$. From Neumann boundary condition $\partial_{n} \varphi_{a}=0$ and oddness in velocity integral, $\int_{\gamma} \psi_{a} P_{\gamma} f(v \cdot n(s))=0$ and we obtain similar esimate as (7.14),

$$
\begin{aligned}
& \int_{s}^{t} \int_{\gamma} \psi_{a} f(v \cdot n(x))=\int_{s}^{t} \int_{\gamma} \psi_{a} \mathbf{1}_{\gamma_{+}}\left(1-P_{\gamma}\right) f d \gamma \\
& \lesssim \varepsilon \int_{s}^{t}\|a(\tau)\|_{2}^{2}+C_{\varepsilon} \int_{s}^{t}\left|\left(1-P_{\gamma}\right) f(\tau)\right|_{2,+}^{2}, \quad \varepsilon \ll 1 .
\end{aligned}
$$

For $(7.7)_{T}$, from oddness,

$$
\begin{aligned}
\left.\left({ }^{(7.7)}\right)_{T}\right|_{\psi_{a}} & =\int_{s}^{t} \iint_{\Omega \times \mathbb{R}^{3}} f \partial_{t} \psi_{a}=\int_{s}^{t} \iint_{\Omega \times \mathbb{R}^{3}}[\sqrt{\mu}(b \cdot v)+(\mathbf{I}-\mathbf{P}) f] \partial_{t} \psi_{a} \\
& \lesssim \varepsilon \int_{s}^{t}\left\|\nabla \Delta^{-1} \partial_{t} a(\tau)\right\|_{2}^{2}+\int_{65}^{t}\|b(\tau)\|_{2}^{2}+\int_{s}^{t}\|(\mathbf{I}-\mathbf{P}) f(\tau)\|_{\nu}^{2} .
\end{aligned}
$$


Now let us estimate $\int_{s}^{t}\left\|\nabla \Delta^{-1} \partial_{t} a(\tau)\right\|_{2}^{2}$ which appear in (17.17) type estimate. We use new test function $\psi_{a}^{t}=$ $\bar{\varphi}(x) \sqrt{\mu}$. It easy to check

$$
\begin{aligned}
& \text { (17.7) }\left.\phi_{f}\right|_{\psi=\sqrt{\mu}}=\int_{s}^{t} \iint_{\Omega \times \mathbb{R}^{3}} \sqrt{\mu} v \cdot \nabla_{x} \phi_{f} \bar{\varphi}(x) \sqrt{\mu}=0, \\
& \left.(17.7)_{P}\right|_{\psi=\sqrt{\mu}}=\int_{s}^{t} \iint_{\Omega \times \mathbb{R}^{3}} \sqrt{\mu} f \nabla_{x} \phi_{f} \cdot \nabla_{v} \bar{\varphi}(x)=0,
\end{aligned}
$$

and from taking difference quotient and null condition (1.7), we obtain, for almost $t$,

$$
\int_{\Omega} \bar{\varphi} \partial_{t} a=\sqrt{2 \pi} \int_{\Omega}\left(b \cdot \nabla_{x}\right) \bar{\varphi} .
$$

In particular, if we choose $\bar{\varphi}=1$, we directly get $\int_{\Omega} \partial_{t} a=0$, so Neumann problem

$$
-\Delta \Phi_{a}=\partial_{t} a(t),\left.\quad \frac{\partial \Phi_{a}}{\partial n}\right|_{\partial \Omega}=0
$$

is well-defined. For dual pair $\left(H^{1}\right)^{*} \equiv\left(H^{1}(\Omega)\right)^{*}$ with respect to $\langle A, B\rangle=\int_{\Omega} A \cdot B d x$ for $A \in H^{1}$ and $B \in\left(H^{1}\right)^{*}$,

$$
\left\|\nabla_{x} \partial_{t} \varphi_{a}\right\|_{2}=\left\|\Delta^{-1} \partial_{t} a(t)\right\|_{H^{1}}=\left\|\Phi_{a}\right\|_{H^{1}} \lesssim\left\|\partial_{t} a(t)\right\|_{\left(H^{1}\right)^{*}} \lesssim\|b(t)\|_{2} .
$$

We change $c$ into $a$ in (7.13) and (17.16) and combine with (7.7), (77.11), (7.12), (7.21), (7.22), and (7.24) with $\varepsilon \ll 1$ to obtain

$$
\begin{aligned}
& \int_{s}^{t}\|a(\tau)\|_{2}^{2}+\int_{s}^{t}\left\|\nabla \phi_{f}(\tau)\right\|_{2}^{2} \\
& \lesssim G_{a}(t)-G_{a}(s)+\int_{s}^{t}\|(\mathbf{I}-\mathbf{P}) f(\tau)\|_{\nu}^{2}+\int_{s}^{t}\left|\left(1-P_{\gamma}\right) f(\tau)\right|_{2,+}^{2} \\
& \quad+\int_{s}^{t}\left\|\nu^{-1 / 2} \Gamma(f, f)(\tau)\right\|_{2}^{2}+\int_{s}^{t}\|w f(\tau)\|_{\infty}^{2}\|\mathbf{P} f(\tau)\|_{2}^{2}+\int_{s}^{t}\|b(\tau)\|_{2}^{2},
\end{aligned}
$$

for $\varepsilon \ll 1$ where $G_{a}(t):=\iint_{\Omega \times \mathbb{R}^{3}} f(t) \psi_{c}(t) \lesssim\|f(t)\|_{2}^{2}$.

Step 4. Estimate of $b:$ For fixed $i, j$, we choose test function $\psi=\psi_{b, 1}^{i, j}$ in (7.8) where $\beta_{b}$ and $\varphi_{b}$ are defined in (7.10) and (7.9). From oddness in velocity integration and definition of $\beta_{b},(7.7)_{C}$ in (7.7) yields

$$
\text { (7.7) }\left.C\right|_{\psi_{b, 1}^{i, j}}:=\int_{s}^{t} \iint_{\Omega \times \mathbb{R}^{3}} \mathbf{P} f v \cdot \nabla \psi_{b, 1}^{i, j}=C_{3} \int_{s}^{t} \int_{\Omega} b_{i}\left(\partial_{i j} \Delta^{-1} b_{j}\right),
$$

where $C_{3}:=\int_{\mathbb{R}^{3}}\left(v_{i}^{2}-\beta_{b}\right) v_{i}^{2} \mu d v=2 \sqrt{2 \pi}$. For boundary integration, contribution of $P_{\gamma} f$ vanishes by oddness.

$$
\begin{aligned}
\left.(\sqrt{7.77})_{B}\right|_{\psi_{b, 1}^{i, j}} & :=\int_{s}^{t} \int_{\gamma} \psi_{b, 1}^{i, j} \mathbf{1}_{\gamma_{+}}\left(1-P_{\gamma}\right) f(v \cdot n(x)) \\
& \lesssim \varepsilon \int_{s}^{t}\|b(\tau)\|_{2}^{2}+\int_{s}^{t}\left|\left(1-P_{\gamma}\right) f\right|_{2,+}^{2},
\end{aligned}
$$

and similar as (7.17) and (7.16), we use oddness and definition of $\beta_{b}$ to vanish contribution of $a$ and $b$. We obtain

$$
\begin{aligned}
& \text { (17.7) }\left.\right|_{\psi_{b, 1}^{i, j}} \\
& \lesssim \varepsilon \int_{s}^{t}\left\|\nabla \Delta^{-1} \partial_{t} b_{j}(\tau)\right\|_{2}^{2}+\int_{s}^{t}\|c(\tau)\|_{2}^{2}+\int_{s}^{t}\|(\mathbf{I}-\mathbf{P}) f(\tau)\|_{\nu}^{2}, \\
& \left.\int_{s}^{t} \iint_{\Omega \times \mathbb{R}^{3}} \psi_{b, 1}^{i, j} R e\right|_{\psi_{b, 1}^{i, j}} \lesssim \varepsilon \int_{s}^{t}\|b(\tau)\|_{2}^{2}+\int_{s}^{t}\|(\mathbf{I}-\mathbf{P}) f(\tau)\|_{\nu}^{2} \\
& +\int_{s}^{t}\left\|\nu^{-1 / 2} \Gamma(f, f)(\tau)\right\|_{2}^{2} .
\end{aligned}
$$


Next, we try test function $\psi_{b, 2}^{i, j}$ with $i \neq j$ to obtain

$$
\begin{aligned}
& \left.(\sqrt{7.7}) C\right|_{\psi_{b, 2}^{i, j}}:=\int_{s}^{t} \iint_{\Omega \times \mathbb{R}^{3}}(b \cdot v) \sqrt{\mu} v \cdot \nabla \psi_{b, 2}^{i, j} \\
& =C_{4} \int_{s}^{t} \int_{\Omega}\left(b_{j}\left(\partial_{i j} \Delta^{-1} b_{i}\right)+b_{i}\left(\partial_{j j} \Delta^{-1} b_{i}\right)\right) .
\end{aligned}
$$

by oddness in velocity integral where $C_{4}:=7 \sqrt{2 \pi}$. We also have the following three estimates using oddness of velocity integral,

$$
\begin{aligned}
\left.(7.7)_{B}\right|_{\psi_{b, 2}^{i, j}} & :=\int_{s}^{t} \int_{\gamma} \psi_{b, 1}^{i, j} \mathbf{1}_{\gamma_{+}}\left(1-P_{\gamma}\right) f(v \cdot n(x)) \\
& \lesssim \varepsilon \int_{s}^{t}\|b(\tau)\|_{2}^{2}+\int_{s}^{t}\left|\left(1-P_{\gamma}\right) f\right|_{2,+}^{2}, \\
\left.\int_{s}^{t} \iint_{\Omega \times \mathbb{R}^{3}} \psi_{b, 2}^{i, j} R e\right|_{\psi_{b, 2}^{i, j}} & \lesssim \varepsilon \int_{s}^{t}\|b(\tau)\|_{2}^{2}+\int_{s}^{t}\|(\mathbf{I}-\mathbf{P}) f(\tau)\|_{\nu}^{2} \\
& +\int_{s}^{t}\left\|\int_{s}^{t}\right\| \nabla \nu^{-1} \partial_{t} b_{i}(\tau)\left\|_{2}^{2}+\int_{s}^{t}\right\|(\mathbf{I}-\mathbf{P}) f(\tau) \|_{\nu}^{2},
\end{aligned}
$$

To obtain estimate for $\left\|\nabla \Delta^{-1} \partial_{t} b_{j}\right\|_{2}$, we use a test function $\psi_{b, j}^{t}:=v_{j} \sqrt{\mu} \partial_{t} \varphi_{b}^{j}(t, x)$. Note that $\partial_{t} \varphi_{b}^{j}$ solves $-\Delta \partial_{t} \varphi_{b}^{j}=\partial_{t} b_{j}(t, x)$ with $\left.\partial_{t} \varphi_{b}^{j}(t, x)\right|_{\partial \Omega}=0$. We taking difference quotient for $\partial_{t} f$ in (1.15) and with help of Poincaré inequality, we get

$$
\begin{aligned}
\text { (17.7) }\left.\phi_{f}\right|_{\psi=\psi_{b, j}^{t}} & =\int_{s}^{t} \iint_{\Omega \times \mathbb{R}^{3}} v_{j} \sqrt{\mu} \partial_{t} \varphi_{b}^{j} v \cdot \nabla_{x} \phi_{f} \sqrt{\mu} \\
\lesssim & \int_{s}^{t}\left\|\nabla \Delta^{-1} \partial_{t} b_{j}(\tau)\right\|_{2}^{2}+\int_{s}^{t}\|a(\tau)\|_{2}^{2}, \\
\left.(\mathbf{7 . 7}) P\right|_{\psi=\psi_{b, j}^{t}} & \int_{s}^{t} \iint_{\Omega \times \mathbb{R}^{3}} \sqrt{\mu} f \partial_{j} \phi_{f} \cdot \partial_{t} \varphi_{b}^{j} \\
\lesssim & \int_{s}^{t}\left\|\nabla \Delta^{-1} \partial_{t} b_{j}(\tau)\right\|_{2}^{2}+\int_{s}^{t}\|a(\tau)\|_{2}^{2} \\
& +\int_{s}^{t}\|(\mathbf{I}-\mathbf{P}) f\|_{\nu}^{2} .
\end{aligned}
$$

Since $\psi_{b, j}^{t}$ vanishes when it acts with $L f$ and $\Gamma(f, f)$, and boundary integral (7.7) $B$ vanishes by Dirichlet boundary condition of $\varphi_{c}$, from (7.32) and (7.7), we obtain

$$
\begin{aligned}
& \int_{s}^{t} \int_{\Omega} \partial_{t} \varphi_{b}^{j}(\tau, x) \partial_{t} b_{j}(\tau, x) d x=\int_{s}^{t}\left\|\nabla \Delta^{-1} \partial_{t} b_{j}(\tau)\right\|_{2}^{2} \\
& \lesssim \varepsilon \int_{s}^{t}\left\|\nabla \Delta^{-1} \partial_{t} b_{j}(\tau)\right\|_{2}^{2}+\int_{s}^{t}\|a(\tau)\|_{2}^{2}+\int_{s}^{t}\|(\mathbf{I}-\mathbf{P}) f(\tau)\|_{\nu}^{2} .
\end{aligned}
$$

Now we combine (7.7), (7.11), (7.12), (7.26), (77.27), (7.28), (7.29), (77.30), and (7.31) for all $i, j$ with proper constant weights. In particular, we note that RHS of (7.26) is cancelled by the first term on the RHS of (7.30). 
Therefore,

$$
\begin{aligned}
& \int_{s}^{t}\|b(\tau)\|_{2}^{2}=-\sum_{i, j} \int_{s}^{t} \int_{\Omega} b_{i}\left(\partial_{j j} \Delta^{-1} b_{i}\right) \\
& \lesssim G_{b}(t)-G_{b}(s)+\int_{s}^{t}\|(\mathbf{I}-\mathbf{P}) f(\tau)\|_{\nu}^{2}+\int_{s}^{t}\left|\left(1-P_{\gamma}\right) f(\tau)\right|_{2,+}^{2} \\
& \quad+\int_{s}^{t}\left\|\nu^{-1 / 2} \Gamma(f, f)(\tau)\right\|_{2}^{2}+\int_{s}^{t}\left\|w_{\vartheta} f(\tau)\right\|_{\infty}^{2}\|\mathbf{P} f(\tau)\|_{2}^{2} \\
& \quad+\int_{s}^{t}\|c(\tau)\|_{2}^{2}+\varepsilon \int_{s}^{t}\|a(\tau)\|_{2}^{2}, \quad G_{b}(t) \lesssim\|f(t)\|_{2}^{2}, \quad \varepsilon \ll 1 .
\end{aligned}
$$

Finally we combine (7.20), (7.25), and (7.34) with $\varepsilon \ll 1$ to conclude (7.3).

\section{Global Existence and Exponential Decay}

We start the section by proving a crucial interpolation in Hölder spaces.

Proof of Lemma 1. Let $\Omega_{1}$ be an open bounded subset of $\mathbb{R}^{3}$ containing the closure $\bar{\Omega}$. Suppose $\phi(t) \in$ $C^{2, D_{2}}(\Omega)$. From a standard extension theorem (e.g. see Lemma 6.37 of 11 in page 136) there exists a function $\bar{\phi}(t) \in C^{2, D_{2}}\left(\Omega_{1}\right)$ and $\bar{\phi}(t) \equiv 0$ in $\mathbb{R}^{3} \backslash \Omega_{1}$ such that $\phi(t) \equiv \bar{\phi}(t)$ in $\Omega$ and

$$
\begin{aligned}
&\|\bar{\phi}(t)\|_{C^{1,1-D_{1}\left(\Omega_{1}\right)}} \leq C_{\Omega, \Omega_{1}, D_{1}, D_{2}}\|\phi(t)\|_{C^{1,1-D_{1}}(\Omega)}, \\
& \text { and }\|\bar{\phi}(t)\|_{C^{2, D_{2}\left(\Omega_{1}\right)}} \leq C_{\Omega, \Omega_{1}, D_{1}, D_{2}}\|\phi(t)\|_{C^{2, D_{2}(\Omega)}},
\end{aligned}
$$

where $C_{\Omega, \Omega_{1}, D_{1}, D_{2}}$ does not depend on $\phi(t)$ and $t$.

Choose arbitrary points $x, y$ in $\mathbb{R}^{3}$. For $0 \leq s \leq 1$, we have $(1-s) x+s y \in \overline{x y}$. Then we derive that

$$
\begin{aligned}
& {[(y-x) \cdot \nabla] \nabla \bar{\phi}(t,(1-s) x+s y) } \\
= & \frac{[(y-x) \cdot \nabla] \nabla \bar{\phi}(t,(1-s) x+s y)-[(y-x) \cdot \nabla] \nabla \bar{\phi}(t, x)}{|(1-s) x+s y-x|^{D_{2}}} \\
& \times|(1-s) x+s y-x|^{D_{2}} \\
+ & \left(\frac{y-x}{|y-x|} \cdot \nabla\right) \nabla \bar{\phi}(t, x)|y-x| \\
= & O\left(|x-y|^{1+D_{2}}\right) s^{D_{2}}\left[\nabla^{2} \bar{\phi}(t)\right]_{C^{0, D_{2}}}+\left(\frac{y-x}{|y-x|} \cdot \nabla\right) \nabla \bar{\phi}(t, x)|y-x| .
\end{aligned}
$$

Taking an integration on $s \in[0,1]$, we obtain that

$$
\begin{aligned}
& \left|\left(\frac{y-x}{|y-x|} \cdot \nabla\right) \nabla \bar{\phi}(t, x)\right| \\
\leq & \frac{1}{|y-x|}\left|\int_{0}^{1}[(y-x) \cdot \nabla] \nabla \bar{\phi}(t,(1-s) x+s y) \mathrm{d} s\right| \\
& +\frac{1}{1+D_{2}}|x-y|^{D_{2}}\left[\nabla^{2} \bar{\phi}(t)\right]_{C^{0, D_{2}}} .
\end{aligned}
$$

On the other hand, from an expansion along $s$,

$$
\nabla \bar{\phi}(t, y)-\nabla \bar{\phi}(t, x)=\int_{0}^{1}[(y-x) \cdot \nabla] \nabla \bar{\phi}(t,(1-s) x+s y) \mathrm{d} s .
$$

We plug this identity into (8.2) and deduce that for $0<D_{1}<1$

$$
\begin{aligned}
& \left|\left(\frac{x-y}{|x-y|} \cdot \nabla\right) \nabla \bar{\phi}(t, x)\right| \\
\leq & \frac{|\nabla \bar{\phi}(t, x)-\nabla \bar{\phi}(t, y)|}{|x-y|}+\frac{1}{1+D_{2}}|x-y|^{D_{2}}\left[\nabla^{2} \bar{\phi}(t)\right]_{C_{x}^{0, D_{2}}} \\
\leq & \frac{1}{|x-y|^{D_{1}}}[\nabla \bar{\phi}(t)]_{C^{0,1-D_{1}}}+\frac{1}{1+D_{2}}|x-y|^{D_{2}}\left[\nabla^{2} \bar{\phi}(t)\right]_{C_{x}^{0, D_{2}}} .
\end{aligned}
$$


Now let us choose

$$
|x-y|=e^{-\Lambda_{0} t}, \quad \hat{\omega}:=\frac{x-y}{|x-y|} \in \mathbb{S}^{2} .
$$

From 8.3

$$
|(\hat{\omega} \cdot \nabla) \nabla \bar{\phi}(t, x)| \leq e^{D_{1} \Lambda_{0} t}[\nabla \bar{\phi}(t)]_{C^{0,1-D_{1}}}+\frac{1}{1+D_{2}} e^{-D_{2} \Lambda_{0} t}\left[\nabla^{2} \bar{\phi}(t)\right]_{C_{x}^{0, D_{2}}} .
$$

Taking supremum in $x$ and $\hat{\omega}$ to the above inequality and using

$$
\left\|\nabla_{x}^{2} \bar{\phi}(t)\right\|_{L_{x}^{\infty}}=\sup _{x} \sup _{\hat{\omega} \in \mathbb{S}^{2}}|(\hat{\omega} \cdot \nabla) \nabla \bar{\phi}(t, x)|,
$$

we get

$$
\begin{aligned}
\left\|\nabla_{x}^{2} \bar{\phi}(t)\right\|_{L^{\infty}\left(\Omega_{1}\right)} \leq & e^{D_{1} \Lambda_{0} t}\left[\nabla_{x} \bar{\phi}(t)\right]_{C^{0,1-D_{1}}\left(\Omega_{1}\right)} \\
& +e^{-D_{2} \Lambda_{0} t}\left[\nabla^{2} \bar{\phi}(t)\right]_{C^{0, D_{2}}\left(\Omega_{1}\right)} .
\end{aligned}
$$

Finally from (8.1) and the above estimate we conclude (1.46).

Now we are ready to prove the global-in-time result.

Proof of Theorem 1. Step 1. For $0<M \ll 1$ and $0<\delta_{*} \ll 1$, we first assume that an initial datum satisfies

$$
\begin{array}{r}
\left\|w_{\vartheta} f_{0}\right\|_{\infty}+\left\|w_{\tilde{\vartheta}} f_{0}\right\|_{p}+\left\|w_{\tilde{\vartheta}} \alpha_{f_{0}, \varepsilon}^{\beta} \nabla_{x, v} f_{0}\right\|_{p} \leq \delta_{*} M, \\
\left\|w_{\tilde{\vartheta}} \nabla_{v} f_{0}\right\|_{L^{3}\left(\Omega \times \mathbb{R}^{3}\right)}+\left\|\nabla_{x}^{2} \phi_{f}(0)\right\|_{\infty}<\infty .
\end{array}
$$

We will choose $M, \delta_{*}$ later. For the sake of convenience we choose a large constant $L \gg \max \left(M,\left\|\nabla_{x}^{2} \phi_{f}(0)\right\|_{\infty}\right)$. In order to use the continuation argument along the lines of the local existence theorem, Theorem 7 we set

$$
\begin{aligned}
& T=\sup _{t}\left\{t \geq 0:\left\|e^{\lambda_{\infty} s} w_{\vartheta} f(t)\right\|_{\infty}+\left\|w_{\tilde{\vartheta}} f(t)\right\|_{p} \leq M,\right. \\
& \quad \text { and }\left\|w_{\tilde{\vartheta}} \alpha_{f, \varepsilon}^{\beta} \nabla_{x, v} f(t)\right\|_{p}^{p}+\int_{0}^{t}\left|w_{\tilde{\vartheta}} \alpha_{f, \varepsilon}^{\beta} \nabla_{x, v} f(t)\right|_{p,+}^{p}<\infty, \\
& \quad \text { and }\left\|\nabla_{v} f(t)\right\|_{L_{x}^{3}(\Omega) L_{v}^{1+\delta}\left(\mathbb{R}^{3}\right)}<\infty, \\
& \text { and } \left.\left\|\nabla_{x}^{2} \phi_{f}(t)\right\|_{\infty} \leq L\right\} .
\end{aligned}
$$

Here for fixed $\delta \ll 1$, we choose $\lambda_{\infty}$ such that

$$
20 \sqrt{C C_{2} M} \leq \lambda_{\infty} \leq \min \left(\frac{\lambda_{2}}{2}, \frac{\nu_{0}}{4}\right), \text { for } M \ll 1,
$$

where $\lambda_{2}$ is obtained in Proposition 8, Note that from (4.6) the condition (4.4) holds for $M \ll 1$.

Step 2. We claim that

$$
\sup _{0 \leq t \leq T} e^{\frac{\lambda \infty}{2} t}\left\|\nabla_{x}^{2} \phi_{f}(t)\right\|_{\infty} \leq C_{2} M, \text { with } C_{2}:=C_{\Omega}+\left(C_{1} C_{p}\right)^{1 / p} \delta_{*} .
$$

Here $C_{\Omega}$ appears in (4.6), and $C_{1}$ in (4.7), and $C_{p}$ in Proposition 4 ,

From (4.6) and (8.5), for $0 \leq t \leq T$, for all $D_{1}>0$

$$
\left\|\phi_{f}(t)\right\|_{C^{1,1-D_{1}}(\bar{\Omega})} \leq C_{\Omega}\left\|w_{\vartheta} f(t)\right\|_{\infty} \leq C_{\Omega} M e^{-\lambda_{\infty} t} .
$$

On the other hand, from Proposition 4. (4.5), and (8.4), we derive that for $0 \leq t \leq T$

$$
\begin{aligned}
& \|f(t)\|_{p}^{p}+\left\|w_{\tilde{\vartheta}} \alpha_{f, \varepsilon}^{\beta} \nabla_{x, v} f(t)\right\|_{p}^{p}+\int_{0}^{t}\left|w_{\tilde{\vartheta}} \alpha_{f, \varepsilon}^{\beta} \nabla_{x, v} f(s)\right|_{p,+}^{p} \\
\leq & C_{p} e^{C_{p}(1+L) t} \times\left(\delta_{*} M\right)^{p} .
\end{aligned}
$$

Now we use Lemma 12, from (4.7), for $p>3$ and $0 \leq t \leq T$,

$$
\left\|\phi_{f}(t)\right\|_{C^{2,1-\frac{3}{p}}(\bar{\Omega})} \leq\left(C_{1} C_{p}\right)^{1 / p} e^{\frac{1}{p} C_{p}(1+L) t} \times \delta_{*} M .
$$


Finally we use an interpolation between $C^{1,1-D_{1}}(\bar{\Omega})$ and $C^{2,1-\frac{3}{p}}(\bar{\Omega})$ and derive an estimate of $C^{2}(\bar{\Omega})$ : Applying Lemma 1 and (1.46) with $D_{2}=1-\frac{3}{p}$, from (8.9) and (8.8), we derive that for all $0<D_{1}<1,3<p<6$, $\Lambda_{0}>0$, and $0 \leq t \leq T$,

$$
\begin{aligned}
\left\|\nabla_{x}^{2} \phi_{f}(t)\right\|_{\infty} \leq & e^{-\left[\lambda_{\infty}-D_{1} \Lambda_{0}\right] t} C_{\Omega} M \\
& +e^{-\left[\left(1-\frac{3}{p}\right) \Lambda_{0}-\frac{1}{p} C_{p}(1+L)\right] t}\left(C_{1} C_{p}\right)^{1 / p} \delta_{*} M .
\end{aligned}
$$

Then we choose

$$
\Lambda_{0}=\frac{\frac{\lambda_{\infty}}{2}+\frac{C_{p}}{p}(1+L)}{1-\frac{3}{p}} \text { and then } D_{1}=\frac{\lambda_{\infty}}{2 \Lambda_{0}} .
$$

In conclusion we have, for all $0 \leq t \leq T$,

$$
\left\|\nabla_{x}^{2} \phi_{f}(t)\right\|_{\infty} \leq e^{-\frac{\lambda_{\infty}}{2} t}\left[C_{\Omega}+\left(C_{1} C_{p}\right)^{1 / p} \delta_{*}\right] M .
$$

As long as $M \ll L$ then $\left\|\nabla_{x}^{2} \phi_{f}(t)\right\|_{\infty} \leq L$ for all $0 \leq t \leq T$ and hence the claim (8.7) holds.

Step 3. We claim that there exists $T_{\infty} \gg 1$ such that, for $N \in \mathbb{N}, t \in\left[N T_{\infty},(N+1) T_{\infty}\right]$, and $(N+1) T_{\infty} \leq T$,

$$
\begin{aligned}
& \quad\left\|w_{\vartheta} f(t)\right\|_{\infty} \\
& \leq e^{-\frac{\nu_{0}}{2}(t-N T)}\left\|w_{\vartheta} f\left(N T_{\infty}\right)\right\|_{\infty} \\
& \quad+o(1) \sup _{N T_{\infty} \leq s \leq t} e^{-\frac{\nu_{0}}{2}(t-s)}\left\|w_{\vartheta} f(s)\right\|_{\infty} \\
& \quad+C_{T_{\infty}} \int_{N T_{\infty}}^{t} e^{-\frac{\nu_{0}}{2}(t-s)}\|f(s)\|_{L_{x, v}^{2}} \mathrm{~d} s \\
& \quad+C_{T_{\infty}} \int_{N T_{\infty}}^{t} e^{-\frac{\nu_{0}}{2}(t-s)}\left\|\nabla \phi_{f}(s)\right\|_{\infty} \mathrm{d} s .
\end{aligned}
$$

For the sake of simplicity we present a proof of (8.13) for $N=0$. The proof for $N>0$ can be easily obtained by considering $f\left(N T_{\infty}\right)$ as an initial datum.

As (6.12) we define $h(t, x, v):=w_{\vartheta} f(t, x, v)$. Then $h$ solves (6.14) and (6.15) with exchanging all $\left(h^{\ell}, h^{\ell+1}, \phi^{\ell}\right)$ to $\left(h, h, \phi_{f}\right)$. We define

$$
\nu_{\phi_{f}, w_{\vartheta}}:=\nu(v)+\frac{v}{2} \cdot \nabla_{x} \phi_{f}-\frac{\nabla_{x} \phi_{f} \cdot \nabla_{\vartheta} w_{\vartheta}}{w_{\vartheta}} .
$$

From (8.5) and (4.6), for $0 \leq t \leq T$

$$
\begin{aligned}
\nu_{\phi_{f}, w_{\vartheta}} & \geq\left\{\nu_{0}-\frac{\left\|\nabla \phi_{f}\right\|_{\infty}}{2}-2 \vartheta\left\|\nabla \phi_{f}\right\|_{\infty}\right\}\langle v\rangle \\
& \geq\left\{\nu_{0}-\left(\frac{1}{2}-2 \vartheta\right) M\right\}\langle v\rangle \\
& \geq \frac{\nu_{0}}{2}\langle v\rangle .
\end{aligned}
$$

Then $h$ solves (6.21) along the trajectory with deleting all superscriptions of $\ell$ and $\ell+1$ and exchanging $\nu^{\ell}$ to $\nu_{\phi_{f}, w_{\vartheta}}$ and with new $g$

We define a stochastic cycles

$$
g:=-v \cdot \nabla \phi_{f} \sqrt{\mu}+\Gamma\left(\frac{h}{w_{\vartheta}}, \frac{h}{w_{\vartheta}}\right) .
$$

$$
\left(t_{l}\left(t, x, v, v_{1}, \cdots, v_{l-1}\right), x_{l}\left(t, x, v, v_{1}, \cdots, v_{l-1}\right)\right),
$$

by deleting all superscriptions in (6.22) and (6.23). Then $h$ has a bound (6.39) with deleting all superscriptions of $\ell$ and $\ell+1$.

For any large $m \gg 1$ we define

$$
\mathbf{k}_{\varrho, m}(v, u)=\mathbf{1}_{|v-u| \geq \frac{1}{m},|v| \leq m} \mathbf{k}_{\varrho}(v, u),
$$

such that $\sup _{v} \int_{\mathbb{R}^{3}}\left|\mathbf{k}_{\varrho, m}(v, u)-\mathbf{k}_{\varrho}(v, u)\right| \mathrm{d} u \lesssim \frac{1}{m}$, and $\left|\mathbf{k}_{\varrho, m}(v, u)\right| \lesssim_{m} 1$.

Furthermore we split the time interval as, for each $\ell, l$

$$
\begin{aligned}
&\left\{\max \left\{t_{l+1}, 0\right\} \leq s \leq t_{l}\right\} \\
&=\left\{\max \left\{t_{l+1}, 0\right\} \leq s \leq t_{l}-\delta\right\} \cup\left\{t_{l}-\delta \leq s \leq t_{l}\right\}, \\
& 70
\end{aligned}
$$


where we choose a small constant $0<\delta \ll_{k} 1$ later in (8.22).

Following (6.39) with an extra decomposition of (8.17), we derive that

$$
\begin{aligned}
&|h(t, x, v)| \\
& \leq O(k)\left\|e^{-\frac{\nu_{0}}{2} t} h_{0}\right\|_{\infty} \\
&+ O(k) \sup _{0 \leq s \leq t}\left\|e^{-\frac{\nu_{0}}{2}(t-s)} h(s)\right\|_{\infty}^{2} \\
&+ O(k) \int_{0}^{t}\left\|e^{-\frac{\nu_{0}}{2}(t-s)} \nabla \phi_{\frac{h}{w_{\vartheta}}}(s)\right\|_{\infty} \mathrm{d} s \\
&+\left\{O(k)\left(\delta+\frac{1}{m}\right)+\left\{\frac{1}{2}\right\}^{k / 5}\right\} \sup _{0 \leq s \leq t}\left\|e^{-\frac{\nu_{0}}{2}(t-s)} h(s)\right\|_{\infty} \\
&+ \int_{\max \left\{t_{1}, 0\right\}}^{t-\delta} e^{-\frac{\nu_{0}}{2}(t-s)} \\
& \quad \times \int_{\mathbb{R}^{3}} \mathbf{k}_{\varrho, m}(V(s ; t, x, v), u)|h(s, X(s ; t, x, v), u)| \mathrm{d} u \mathrm{~d} s \\
&+O(k) \sup _{l}^{t_{l}-\delta} \int_{\max \left\{t_{l+1}, 0\right\}} e^{-\frac{\nu_{0}}{2}(t-s)} \\
& \quad \times \int_{|u| \leq m} \int_{\left|v_{l}\right| \leq m}\left|h\left(s, X\left(s ; t_{l}, x_{l}, v_{l}\right), u\right)\right| \mathrm{d} v_{l} \mathrm{~d} u \mathrm{~d} s .
\end{aligned}
$$

Note that $|h(s, X(s ; t, x, v), u)|$ has the similar bound as

$$
\begin{aligned}
& \quad|h(s, X(s ; t, x, v), u)| \\
& \leq O(k)\left\|e^{-\frac{\nu_{0}}{2} s} h_{0}\right\|_{\infty} \\
& +O(k) \sup _{0 \leq s^{\prime} \leq s}\left\|e^{-\frac{\nu_{0}}{2}\left(s-s^{\prime}\right)} h\left(s^{\prime}\right)\right\|_{\infty}^{2} \\
& +O(k) \int_{0}^{s}\left\|e^{-\frac{\nu_{0}}{2}\left(s-s^{\prime}\right)} \nabla \phi_{\frac{h}{w_{\vartheta}}}\left(s^{\prime}\right)\right\|_{\infty} \mathrm{d} s^{\prime} \\
& +\left\{O(k)\left(\delta+\frac{1}{m}\right)+\left\{\frac{1}{2}\right\}^{k / 5}\right\} \sup _{0 \leq s^{\prime} \leq s}\left\|e^{-\frac{\nu_{0}}{2}\left(s-s^{\prime}\right)} h\left(s^{\prime}\right)\right\|_{\infty} \\
& +\int_{\max \left\{t_{1}^{\prime}, 0\right\}}^{s-\delta} e^{-\frac{\nu_{0}}{2}\left(s-s^{\prime}\right)} \\
& \quad \times \int_{\left|u^{\prime}\right| \leq m}\left|h\left(s^{\prime}, X\left(s^{\prime} ; s, X(s ; t, x, v), u\right), u^{\prime}\right)\right| \mathrm{d} u^{\prime} \mathrm{d} s^{\prime} \\
& +O(k) \sup _{l, l^{\prime}} \int_{\max \left\{t_{l^{\prime}+1}^{\prime}, 0\right\}}^{t_{l^{\prime}}^{\prime}-\delta} e^{-\frac{\nu_{0}}{2}\left(s-s^{\prime}\right)} \\
& \quad \times \int_{\left|u^{\prime}\right| \leq m} \int_{\left|v_{l^{\prime}}^{\prime}\right| \leq m}\left|h\left(s^{\prime}, X\left(s^{\prime} ; t_{l^{\prime}}^{\prime}, x_{l^{\prime}}^{\prime}, v_{l^{\prime}}^{\prime}\right), u^{\prime}\right)\right| \mathrm{d} v_{l^{\prime}}^{\prime} \mathrm{d} u^{\prime} \mathrm{d} s^{\prime},
\end{aligned}
$$

where

$$
\begin{aligned}
t_{l^{\prime}}^{\prime} & =t_{l^{\prime}}\left(s, X(s ; t, x, v), u, v_{1}^{\prime}, \cdots, v_{l^{\prime}-1}^{\prime}\right), \\
x_{l^{\prime}}^{\prime} & =x_{l^{\prime}}\left(s, X(s ; t, x, v), u, v_{1}^{\prime}, \cdots, v_{l^{\prime}-1}^{\prime}\right) .
\end{aligned}
$$


Plugging (8.20) into (8.19) we conclude that

$$
\begin{aligned}
& |h(t, x, v)| \\
\leq & O_{m}(k)\left\{\left\|e^{-\frac{\nu_{0}}{2} t} h_{0}\right\|_{\infty}+\sup _{0 \leq s \leq t}\left\|e^{-\frac{\nu_{0}}{2}(t-s)} h(s)\right\|_{\infty}^{2}\right\} \\
& +O_{m}(k) \int_{0}^{t}\left\|e^{-\frac{\nu_{0}}{2}(t-s)} \nabla \phi_{\frac{h}{w_{\vartheta}}}(s)\right\|_{\infty} \mathrm{d} s \\
& +\left\{O_{m}(k) \delta+O(k) \frac{1}{m}+O_{m}(1)\left\{\frac{1}{2}\right\}^{k / 5}\right\} \sup _{0 \leq s \leq t}\left\|e^{-\frac{\nu_{0}}{2}(t-s)} h(s)\right\|_{\infty} \\
+ & \int_{0}^{t} \int_{\left|u^{\prime}\right| \leq m} \int_{0}^{s-\delta} e^{-\frac{\nu_{0}}{2}\left(t-s^{\prime}\right)} \int_{|u| \leq m} \\
& \times\left|h\left(s^{\prime}, X\left(s^{\prime} ; s, X(s ; t, x, v), u\right), u^{\prime}\right)\right| \mathrm{d} u \mathrm{~d} s^{\prime} \mathrm{d} u^{\prime} \mathrm{d} s \\
+ & O(k) \sup _{l} \int_{0}^{t_{l}-\delta} e^{-\frac{\nu_{0}}{2}(t-s)} \\
& \times \int_{|u| \leq m} \int_{\left|v_{l}\right| \leq m}\left|h\left(s, X\left(s ; t_{l}, x_{l}, v_{l}\right), u\right)\right| \mathrm{d} v_{l} \mathrm{~d} u \mathrm{~d} s \\
+ & O(k) \sup _{l, l^{\prime}} \int_{0}^{t} \int_{|u| \leq m} \int_{0}^{t_{l^{\prime}}^{\prime}-\delta} e^{-\frac{\nu_{0}}{2}\left(t-s^{\prime}\right)} \\
& \times \int_{\left|u^{\prime}\right| \leq m} \int_{\left|v_{l^{\prime}}^{\prime}\right| \leq m}\left|h\left(s^{\prime}, X\left(s^{\prime} ; t_{l^{\prime}}^{\prime}, x_{l^{\prime}}^{\prime}, v_{l^{\prime}}^{\prime}\right), u^{\prime}\right)\right| \mathrm{d} v_{l^{\prime}}^{\prime} \mathrm{d} u^{\prime} \mathrm{d} s^{\prime} .
\end{aligned}
$$

Choose $T \gg 1$ and $k \gg 1$ in (6.34) and (6.36). Then we choose

$$
m=k^{2} \text { and } \delta=\frac{1}{m^{3} k},
$$

so that $O_{m}(k) \delta+O(k) \frac{1}{m}+O_{m}(1)\left\{\frac{1}{2}\right\}^{k / 5} \ll 1$.

Note that

$$
\begin{aligned}
& \frac{\partial X\left(s ; t_{l}, x_{l}, v_{l}\right)}{\partial v_{l}} \\
= & -\left(t_{l}-s\right) \operatorname{Id}_{3 \times 3} \\
& -\int_{t_{l}}^{s} \int_{t_{l}}^{\tau} \frac{\partial X\left(\tau^{\prime} ; t_{l}, x_{l}, v_{l}\right)}{\partial v_{l}} \cdot \nabla_{x}\left(\nabla_{x} \phi_{\frac{h}{w_{\vartheta}}}\left(\tau^{\prime}, X\left(\tau^{\prime} ; t_{l}, x_{l}, v_{l}\right)\right)\right) \mathrm{d} \tau^{\prime} \mathrm{d} \tau .
\end{aligned}
$$

Now we use Lemma 10. Note that from (8.7), the condition (3.1) of Lemma 10 is satisfied with $\Lambda_{2}=\frac{\lambda_{\infty}}{2}$ and $\delta_{2}=C_{2} M$. From Lemma 10 and (3.7) we have

$$
\left|\frac{\partial X\left(\tau^{\prime} ; t_{l}, x_{l}, v_{l}\right)}{\partial v_{l}}\right| \leq C e^{\frac{4 C C_{2} M}{\left(\lambda_{\infty}\right)^{2}}}\left|t_{l}-\tau^{\prime}\right|
$$

From (8.24) and (8.7), the second term of RHS in (8.23) is bounded by

$$
\begin{aligned}
& C C_{2} M e^{\frac{4 C C_{2} M}{\left(\lambda_{\infty}\right)^{2}}} \int_{s}^{t_{l}} \int_{\tau}^{t_{l}}\left(t_{l}-\tau^{\prime}\right) e^{-\frac{\lambda \infty}{2} \tau^{\prime}} \mathrm{d} \tau^{\prime} \mathrm{d} \tau \\
\leq & \frac{4 C C_{2} M}{\left(\lambda_{\infty}\right)^{2}} e^{\frac{4 C C_{2} M}{\left(\lambda_{\infty}\right)^{2}}}\left|t_{l}-s\right|,
\end{aligned}
$$

where we have used (3.12). From our choice of $\lambda_{\infty}$ in (8.6), we have

$$
\frac{4 C C_{2} M}{\left(\lambda_{\infty}\right)^{2}} e^{\frac{4 C C_{2} M}{\left(\lambda_{\infty}\right)^{2}}}<\frac{1}{10}
$$


Therefore from (8.23), for $0 \leq s \leq t_{l}-\delta$

$$
\begin{aligned}
& \operatorname{det}\left(\frac{\partial X\left(s ; t_{l}, x_{l}, v_{l}\right)}{\partial v_{l}}\right) \\
= & \operatorname{det}\left(-\left(t_{l}-s\right) \operatorname{Id}_{3 \times 3}+o(1)\right) \\
\gtrsim & \left|t_{l}-s\right|^{3} \\
\gtrsim & \delta^{3} .
\end{aligned}
$$

We can obtain the exactly same lower bound for both

$$
\begin{aligned}
& \operatorname{det}\left(\frac{\partial X\left(s ; t_{l^{\prime}}^{\prime}, x_{l^{\prime}}^{\prime}, v_{l^{\prime}}^{\prime}\right)}{\partial v_{l^{\prime}}^{\prime}}\right) \text { for } 0 \leq s^{\prime} \leq s-\delta \\
& \operatorname{det}\left(\frac{\partial X\left(s^{\prime} ; s, X(s ; t, x, v), u\right)}{\partial u}\right) \text { for } 0 \leq s^{\prime} \leq t_{l^{\prime}}^{\prime}-\delta .
\end{aligned}
$$

Now we apply the change of variables

$$
\begin{aligned}
v_{l} & \mapsto X\left(s ; t_{l}, x_{l}, v_{l}\right), \\
v_{l^{\prime}}^{\prime} & \mapsto X\left(s ; t_{l^{\prime}}^{\prime}, x_{l^{\prime}}^{\prime}, v_{l^{\prime}}^{\prime}\right), \\
u & \mapsto X\left(s^{\prime} ; s, X(s ; t, x, v), u\right),
\end{aligned}
$$

and conclude (8.13) from (8.21) and (8.22).

Applying (8.13) successively, we achieve that

$$
\begin{aligned}
& \left\|w_{\vartheta} f(t)\right\|_{\infty} \\
\leq & e^{\frac{\nu_{0}}{2}} e^{-\frac{\nu_{0}}{2} t}\left\|w_{\vartheta} f(0)\right\|_{\infty}+o(1) \frac{e^{\nu_{0} T_{\infty}}}{1-e^{-\nu_{0} T_{\infty}}} \sup _{0 \leq s \leq t} e^{-\nu_{0}(t-s)}\left\|w_{\vartheta} f(s)\right\|_{\infty} \\
+ & \underbrace{\text { 8.27 }_{L^{2}}}_{\underbrace{}_{T_{\infty}} e^{\frac{\nu_{0}}{2}} \int_{0}^{t} e^{-\frac{\nu_{0}}{2}(t-s)}\|f(s)\|_{2} \mathrm{~d} s} \\
+ & \underbrace{C_{T_{\infty}} e^{\frac{\nu_{0}}{2}} \int_{0}^{t} e^{-\frac{\nu_{0}}{2}(t-s)}\left\|\nabla \phi_{f}(s)\right\|_{\infty} \mathrm{d} s}_{T_{\phi_{f}}},
\end{aligned}
$$

where we have used

$$
e^{\nu_{0} T_{\infty}}\left\{1+e^{-\nu_{0} T_{\infty}}+\cdots+e^{-\nu_{0} N T_{\infty}}\right\}=\frac{e^{\nu_{0} T_{\infty}}}{1-e^{-\nu_{0} T_{\infty}}} .
$$

Step 4. From Proposition 8 and (7.1) we have

$$
\begin{aligned}
& \left\|e^{\lambda_{2} t} f(t)\right\|_{2}^{2}+\left\|e^{\lambda_{2} t} \nabla \phi(t)\right\|_{2}^{2} \\
& +\int_{0}^{t}\left\|e^{\lambda_{2} \tau} f(\tau)\right\|_{\nu}^{2}+\left\|e^{\lambda_{2} \tau} \nabla \phi_{f}(\tau)\right\|_{2}^{2} \mathrm{~d} \tau+\int_{0}^{t}\left|e^{\lambda_{2} \tau} f\right|_{2,+}^{2} \\
\lesssim & \left\|f_{0}\right\|_{2}^{2}+\left\|\nabla \phi_{f_{0}}\right\|_{2}^{2} .
\end{aligned}
$$

Hence

$$
\text { (8.27) } \begin{aligned}
L^{2} & \lesssim t e^{-\min \left(\frac{\nu_{0}}{2}, \lambda_{2}\right) \times t}\left\{\left\|f_{0}\right\|_{2}+\left\|\nabla \phi_{f_{0}}\right\|_{2}\right\} \\
& \lesssim e^{-\min \left(\frac{\nu_{0}}{4}, \frac{\lambda_{2}}{2}\right) \times t}\left\{\left\|f_{0}\right\|_{2}+\left\|\nabla \phi_{f_{0}}\right\|_{2}\right\} .
\end{aligned}
$$

Now we consider $(8.27) \phi_{f}$. In order to close the estimate in (8.27) we need to improve the decay rate of $\left\|\nabla \phi_{f}(s)\right\|_{\infty}$. We claim that, for $\theta_{2, r, p}>0$ (which is specified in (8.34)),

$$
\left\|\nabla_{x} \phi_{f}(s)\right\|_{\infty} \lesssim e^{-\left(1+\theta_{2, r, p}\right) \lambda_{\infty} s}\left\{\sup _{t \geq 0}\left\|e^{\lambda_{2} t} f(s)\right\|_{2}+\sup _{t \geq 0}\left\|e^{\lambda_{\infty} t} f(s)\right\|_{\infty}\right\} .
$$

By Morrey's inequality for $\Omega \subset \mathbb{R}^{3}$ and $r>3$

$$
\left\|\nabla_{x} \phi_{f}\right\|_{\infty} \lesssim\left\|\nabla_{x} \phi_{f}\right\|_{C^{0,1-3 / r}(\Omega)} \lesssim\left\|\nabla_{x} \phi_{f}\right\|_{W^{1, r}(\Omega)} .
$$


Then applying the standard elliptic estimate to (1.16), we get

$$
\begin{gathered}
\left\|\nabla_{x} \phi_{f}(t)\right\|_{W^{1,2}(\Omega)} \lesssim\left\|\int_{\mathbb{R}^{3}} f(t) \sqrt{\mu} \mathrm{d} v\right\|_{L^{2}(\Omega)} \lesssim e^{-\lambda_{2} t} \sup _{t \geq 0}\left\|e^{\lambda_{2} t} f(t)\right\|_{2}, \\
\left\|\nabla_{x} \phi_{f}(t)\right\|_{W^{1, p}(\Omega)} \lesssim\left\|\int_{\mathbb{R}^{3}} f(t) \sqrt{\mu} \mathrm{d} v\right\|_{L^{p}(\Omega)} \lesssim e^{-\lambda_{\infty} t} \sup _{t \geq 0}\left\|e^{\lambda_{\infty} t} f(t)\right\|_{\infty} .
\end{gathered}
$$

Now we use the standard interpolation: For $p>r>3$,

$$
\left\|\nabla_{x} \phi_{f}\right\|_{W^{1, r}(\Omega)} \lesssim\left\|\nabla_{x} \phi_{f}(t)\right\|_{W^{1,2}(\Omega)}^{\theta_{2, r, p}}\left\|\nabla_{x} \phi_{f}(t)\right\|_{W^{1, p}(\Omega)}^{1-\theta_{2, r, p}}
$$

for

$$
\theta_{2, r, p}:=\frac{\frac{1}{r}-\frac{1}{p}}{\frac{1}{2}-\frac{1}{p}}>\frac{2}{3} \cdot \frac{p-3}{p-2}
$$

Then we derive

$$
\begin{aligned}
& \sup _{t \geq 0}\left\|e^{\left[\theta_{2, r, p} \lambda_{2}+\left(1-\theta_{2, r, p}\right) \lambda_{\infty}\right] t} \nabla_{x} \phi_{f}(t)\right\|_{\infty} \\
\lesssim & \left(\sup _{t \geq 0}\left\|e^{\lambda_{2} t} f(t)\right\|_{2}\right)^{\theta_{2, r, p}}\left(\sup _{t \geq 0}\left\|e^{\lambda_{\infty} t} f(t)\right\|_{\infty}\right)^{1-\theta_{2, r, p}} \\
\lesssim & \sup _{t \geq 0}\left\|e^{\lambda_{2} t} f(t)\right\|_{2}+o(1) \sup _{t \geq 0}\left\|e^{\lambda_{\infty} t} f(t)\right\|_{\infty} .
\end{aligned}
$$

From our choice (8.6) and $0<p-3 \ll 1$,

$$
\theta_{2, r, p} \lambda_{2}+\left(1-\theta_{2, r, p}\right) \lambda_{\infty} \geq\left(1+\theta_{2, r, p}\right) \lambda_{\infty}
$$

From (8.35)

$$
\begin{aligned}
\text { (8.27) } \phi_{f} \lesssim & \int_{0}^{t} e^{-\frac{\nu_{0}}{2}(t-s)} e^{-\left(1+\theta_{2, r, p}\right) \lambda_{\infty} s}\left\|e^{\lambda_{2} s} f(s)\right\|_{2} \mathrm{~d} s \\
& +o(1) \int_{0}^{t} e^{-\frac{\nu_{0}}{2}(t-s)} e^{-\left(1+\theta_{2, r, p}\right) \lambda_{\infty} s}\left\|e^{\lambda_{\infty} s} w_{\vartheta} f(s)\right\|_{\infty} \mathrm{d} s \\
\lesssim & e^{-\min \left(\frac{\nu_{0}}{4}, \lambda_{\infty}\right) \times t}\left\{\left\|f_{0}\right\|_{2}+\left\|\nabla \phi_{f_{0}}\right\|_{2}\right\} \quad \text { from (8.28) } \\
& +o(1) e^{-\min \left(\frac{\nu_{0}}{4}, \lambda_{\infty}\right) \times t} \sup _{0 \leq s \leq t}\left\|e^{\lambda_{\infty} s} w_{\vartheta} f(s)\right\|_{\infty} .
\end{aligned}
$$
that

Multiplying $e^{\lambda_{\infty} t}$ and taking $\sup _{t \geq 0}$ to (8.27) with $\lambda_{\infty} \leq \min \left(\frac{\nu_{0}}{4}, \frac{\lambda_{2}}{2}\right)$, and from (8.29) and (8.37), we obtain

$$
\begin{aligned}
\sup _{t \geq 0} e^{\lambda_{\infty} t}\left\|w_{\vartheta} f(t)\right\|_{\infty} \lesssim & \left\|w_{\vartheta} f(0)\right\|_{\infty}+\left\|f_{0}\right\|_{2}+\left\|\nabla \phi_{f_{0}}\right\|_{2} \\
& +o(1) \sup _{0 \leq s \leq t} e^{\lambda_{\infty} s}\left\|w_{\vartheta} f(s)\right\|_{\infty} .
\end{aligned}
$$

By absorbing the last (small) term, we conclude that

$$
\sup _{0 \leq t \leq T} e^{\lambda_{\infty} t}\left\|w_{\vartheta} f(t)\right\|_{\infty} \leq \mathfrak{C} \delta_{*} M
$$

If we choose $\delta_{*} \ll 1 / \mathfrak{C}$ then by the local existence theorem (Theorem 7) and continuity of $\left\|w_{\vartheta} f(t)\right\|_{\infty}$, $\left\|w_{\tilde{\vartheta}} f(t)\right\|_{p}^{p}+\left\|w_{\tilde{\vartheta}} \alpha_{f, \varepsilon}^{\beta} \nabla_{x, v} f(s)\right\|_{p}^{p}+\int_{0}^{t}\left|w_{\tilde{\vartheta}} \alpha_{f, \varepsilon}^{\beta} \nabla_{x, v} f(s)\right|_{p,+}^{p}$, and $\left\|\nabla_{v} f(t)\right\|_{L_{x}^{3}(\Omega) L_{v}^{1+\delta}\left(\mathbb{R}^{3}\right)}$, we conclude that $T=\infty$.

Then the estimates of (1.37), (1.38), and (1.39) are direct consequence of Proposition 4, Lemma 12, Proposition 5 and Proposition 6

\section{Appendix A. Auxiliary Results and Proofs}

Proof of (1.28). From (1.22), for $t-t_{\mathbf{b}}(t, x, v)<s \leq t$,

$$
\begin{aligned}
& x_{\mathbf{b}}(s, X(s ; t, x, v), V(s ; t, x, v))=x_{\mathbf{b}}(t, x, v), \\
& v_{\mathbf{b}}(s, X(s ; t, x, v), V(s ; t, x, v))=v_{\mathbf{b}}(t, x, v) .
\end{aligned}
$$


Therefore

$$
\begin{aligned}
& {\left[\partial_{t}+v \cdot \nabla_{x}-\nabla_{x} \phi_{f} \cdot \nabla_{v}\right] \alpha_{f, \varepsilon}(t, x, v) } \\
= & \left.\frac{d}{d s} \alpha_{f, \varepsilon}(s, X(s ; t, x, v), V(s ; t, x, v))\right|_{s=t} \\
= & \frac{d}{d s} \alpha_{f, \varepsilon}(t, x, v) \\
= & 0 .
\end{aligned}
$$

From (1.23) and (1.22),

$$
t_{\mathbf{b}}(s, X(s ; t, x, v), V(s ; t, x, v))=t_{\mathbf{b}}(t, x, v)-(t-s) .
$$

Therefore

$$
\begin{aligned}
& {\left[\partial_{t}+v \cdot \nabla_{x}-\nabla_{x} \phi_{f} \cdot \nabla_{v}\right]\left(t-t_{\mathbf{b}}(t, x, v)\right) } \\
= & \left.\frac{d}{d s}\left[s-t_{\mathbf{b}}(s, X(s ; t, x, v), V(s ; t, x, v))\right]\right|_{s=t} \\
= & \frac{d}{d s}\left[t-t_{\mathbf{b}}(t, x, v)\right]=0 .
\end{aligned}
$$

These prove (1.28).

Proof of 4.13). The proof follows the argument of Lemma 7 in [17. Note

$$
\mathbf{k}_{\varrho}(v, u) \frac{e^{\vartheta|v|^{2}}}{e^{\vartheta|u|^{2}}}=\frac{1}{|v-u|} \exp \left\{-\varrho|v-u|^{2}-\varrho \frac{\left.|| v\right|^{2}-\left.|u|^{2}\right|^{2}}{|v-u|^{2}}+\vartheta|v|^{2}-\vartheta|u|^{2}\right\} .
$$

Let $v-u=\eta$ and $u=v-\eta$. Then the exponent equals

$$
\begin{aligned}
& -\varrho|\eta|^{2}-\varrho \frac{\left.|| \eta\right|^{2}-\left.2 v \cdot \eta\right|^{2}}{|\eta|^{2}}-\vartheta\left\{|v-\eta|^{2}-|v|^{2}\right\} \\
= & -2 \varrho|\eta|^{2}+4 \varrho v \cdot \eta-4 \varrho \frac{|v \cdot \eta|^{2}}{|\eta|^{2}}-\vartheta\left\{|\eta|^{2}-2 v \cdot \eta\right\} \\
= & (-2 \varrho-\vartheta)|\eta|^{2}+(4 \varrho+2 \vartheta) v \cdot \eta-4 \varrho \frac{\{v \cdot \eta\}^{2}}{|\eta|^{2}} .
\end{aligned}
$$

If $0<\vartheta<4 \varrho$ then the discriminant of the above quadratic form of $|\eta|$ and $\frac{v \cdot \eta}{|\eta|}$ is

$$
(4 \varrho+2 \vartheta)^{2}-4(-2 \varrho-\vartheta)(-4 \varrho)=4 \vartheta^{2}-16 \varrho \vartheta<0 .
$$

Hence, the quadratic form is negative definite. We thus have, for $0<\tilde{\varrho}<\varrho-\frac{\vartheta}{4}$, the following perturbed quadratic form is still negative definite

$$
-(\varrho-\tilde{\varrho})|\eta|^{2}-(\varrho-\tilde{\varrho}) \frac{\left.|| \eta\right|^{2}-\left.2 v \cdot \eta\right|^{2}}{|\eta|^{2}}-\vartheta\left\{|\eta|^{2}-2 v \cdot \eta\right\} \leq 0 .
$$

Therefore we conclude (4.13).

Recall $\kappa_{\delta}(x, v)$ in (6.54). Let us denote $f_{\delta}(t, x, v):=\kappa_{\delta}(x, v) f(t, x, v)$. We assume that $f(s, x, v)=e^{s} f_{0}(x, v)$ for $s<0$. Then

$$
\begin{aligned}
\left\|f_{\delta}\right\|_{L^{2}\left(\mathbb{R} \times \Omega \times \mathbb{R}^{3}\right)} & \lesssim\|f\|_{L^{2}\left(\mathbb{R}_{+} \times \Omega \times \mathbb{R}^{3}\right)}+\left\|f_{0}\right\|_{L^{2}\left(\Omega \times \mathbb{R}^{3}\right)}, \\
\left\|f_{\delta}\right\|_{L^{2}(\mathbb{R} \times \gamma)} & \lesssim\left\|f_{\gamma}\right\|_{L^{2}\left(\mathbb{R}_{+} \times \gamma\right)}+\left\|f_{0}\right\|_{L^{2}(\gamma)} .
\end{aligned}
$$

Lemma 14. Assume $\Omega$ is convex in (1.30) and $\sup _{0 \leq t \leq T}\|E(t)\|_{L^{\infty}(\Omega)}<\infty$. Let $\bar{E}(t, x)=1_{\Omega}(x) E(t, x)$ for $x \in \mathbb{R}^{3}$. There exists $\bar{f}(t, x, v) \in L^{2}\left(\mathbb{R} \times \mathbb{R}^{3} \times \mathbb{R}^{3}\right)$, an extension of $f_{\delta}$, such that

$$
\left.\bar{f}\right|_{\Omega \times \mathbb{R}^{3}} \equiv f_{\delta} \text { and }\left.\left.\bar{f}\right|_{\gamma} \equiv f_{\delta}\right|_{\gamma} \text { and }\left.\left.\bar{f}\right|_{t=0} \equiv f_{\delta}\right|_{t=0} .
$$

Moreover, in the sense of distributions on $\mathbb{R} \times \mathbb{R}^{3} \times \mathbb{R}^{3}$,

$$
\left[\partial_{t}+v \cdot \nabla_{x}+\bar{E} \cdot \nabla_{v}\right] \bar{f}=h_{1}+h_{2},
$$


where

$$
\begin{aligned}
h_{1}(t, x, v)= & \kappa_{\delta}(x, v) \mathbf{1}_{t \in[0, \infty)}\left[\partial_{t}+v \cdot \nabla_{x}+E \cdot \nabla_{v}\right] f \\
& +\kappa_{\delta}(x, v) \mathbf{1}_{t \in(-\infty, 0]} e^{t}\left[1+v \cdot \nabla_{x}+E \cdot \nabla_{v}\right] f_{0} \kappa_{\delta}(x, v) \\
& +f(t, x, v)\left[v \cdot \nabla_{x}+E \cdot \nabla_{v}\right] \kappa_{\delta}(x, v), \\
h_{2}(t, x, v)= & \mathbf{1}_{t-t_{\mathbf{b}}^{E X}(t, x, v) \leq 0} \mathbf{1}_{x_{\mathbf{b}}^{E X}(t, x, v) \in \partial \Omega} e^{t-t_{\mathbf{b}}^{E X}(x, v)} f_{0}\left(x_{\mathbf{b}}^{E X}(x, v), v\right) \\
& +\mathbf{1}_{t-t_{\mathbf{b}}^{E X}(t, x, v) \leq 0} \mathbf{1}_{x_{\mathbf{f}}^{E X}(t, x, v) \in \partial \Omega} e^{t-t_{\mathbf{f}}^{E X}(x, v)} f_{0}\left(x_{\mathbf{f}}^{E X}(x, v), v\right),
\end{aligned}
$$

where $t_{\mathbf{b}}^{E X}, x_{\mathbf{b}}^{E X}, t_{\mathbf{f}}^{E X}, x_{\mathbf{f}}^{E X}$ are defined in A.5.

Moreover,

$$
\begin{aligned}
\left\|h_{1}\right\|_{L^{2}\left(\mathbb{R} \times \mathbb{R}^{3} \times \mathbb{R}^{3}\right)} \lesssim & \left\|\left[\partial_{t}+v \cdot \nabla_{x}+E \cdot \nabla_{v}\right] f\right\|_{L^{2}\left(\mathbb{R}_{+} \times \Omega \times \mathbb{R}^{3}\right)} \\
& +\|f\|_{L^{2}\left(\mathbb{R} \times \Omega \times \mathbb{R}^{3}\right)}+\left\|\left[v \cdot \nabla_{x}+E \cdot \nabla_{v}\right] f_{0}\right\|_{L^{2}\left(\Omega \times \mathbb{R}^{3}\right)} \\
\left\|h_{2}\right\|_{L^{2}\left(\mathbb{R} \times \mathbb{R}^{3} \times \mathbb{R}^{3}\right)} \lesssim & \left\|f_{0}\right\|_{L^{2}(\gamma)} .
\end{aligned}
$$

Proof. In the sense of distributions

$$
\partial_{t} f_{\delta}+v \cdot \nabla_{x} f_{\delta}+E \cdot \nabla_{v} f_{\delta}=h_{1} \text { in } A .2 \text {. }
$$

Clearly $\left|\left[v \cdot \nabla_{x}+E \cdot \nabla_{v}\right] \kappa_{\delta}(x, v)\right| \lesssim_{\delta} 1$.

For $x \in \mathbb{R}^{3} \backslash \bar{\Omega}$ we define

$$
\begin{aligned}
& t_{\mathbf{b}}^{E X}(t, x, v):=\sup \left\{s \geq 0: x-(t-\tau) v \in \mathbb{R}^{3} \backslash \bar{\Omega} \text { for all } \tau \in(t-s, t)\right\}, \\
& t_{\mathbf{f}}^{E X}(t, x, v):=\sup \left\{s \geq 0: x-(t-\tau) v \in \mathbb{R}^{3} \backslash \bar{\Omega} \text { for all } \tau \in(t, t+s)\right\},
\end{aligned}
$$

and $x_{\mathbf{b}}^{E X}(t, x, v)=x-\left(t-t_{\mathbf{b}}^{E X}(t, x, v)\right) v, x_{\mathbf{f}}^{E X}(t, x, v)=x-\left(t-t_{\mathbf{f}}^{E X}(t, x, v)\right) v$.

We define, for $x \in \mathbb{R}^{3} \backslash \bar{\Omega}$,

$$
\begin{aligned}
f_{E}(t, x, v) & =\mathbf{1}_{x_{\mathbf{b}}^{E X}(t, x, v) \in \partial \Omega} f_{\delta}\left(t-t_{\mathbf{b}}^{E X}(t, x, v), x_{\mathbf{b}}^{E X}(t, x, v), v\right) \\
& +\mathbf{1}_{x_{\mathbf{f}}^{E X}(t, x, v) \in \partial \Omega} f_{\delta}\left(t-t_{\mathbf{f}}^{E X}(t, x, v), x_{\mathbf{f}}^{E X}(t, x, v), v\right) .
\end{aligned}
$$

Recall that, from (6.54), $f_{\delta} \equiv 0$ when $v=0$ and hence $f_{E} \equiv 0$ for $v=0$. Since $\Omega$ is convex if $v \neq 0$ then $\left\{x_{\mathbf{b}}^{E X}(t, x, v) \in \partial \Omega\right\} \cap\left\{x_{\mathbf{f}}^{E X}(t, x, v) \in \partial \Omega\right\}=\emptyset$. Note that

$$
f_{E}(t, x, v)=f_{\gamma}(t, x, v)=f_{\delta}(t, x, v) \quad \text { for } x \in \partial \Omega .
$$

In the sense of distribution, in $\mathbb{R} \times\left[\mathbb{R}^{3} \backslash \bar{\Omega}\right] \times \mathbb{R}^{3}$

$$
\partial_{t} f_{E}+v \cdot \nabla_{x} f_{E}=h_{2} \text { in } A .2 \text {. }
$$

We define

$$
\bar{f}(t, x, v):=\mathbf{1}_{\Omega}(x) f_{\delta}(t, x, v)+\mathbf{1}_{\mathbb{R}^{3} \backslash \bar{\Omega}}(x) f_{E}(t, x, v) .
$$

From (A.4), A.7), and (A.8) we prove (A.1). The estimates of (A.3) are direct consequence of Lemma 3 and Lemma 4

Acknowledgements. The authors thank Hongxu Chen to find errors in the original manuscript and fix them. They also thank referee(s) for useful comments which help us to improve the clarity of presentation. The authors thank Yan Guo for his interest and discussions. They also thank Clément Mouhot, Lello Esposito, Rossana Marra, Misha Feldman, Hyung Ju Hwang, and Stéphane Mischler for their interest to this project. C.K. especially thanks James Callen(Center for Plasma Theory and Computation) for discussions on the several relevant kinetic models. The authors also thank kind hospitality of MFO at Oberwolfach, ICERM, KAIST-CMC, math/applied math departments of Brown, Cambridge, Princeton, USC(during a summer school organized by Juhi Jang), UMN, UIC, UT-Austin, POSTECH, NTU, Lyon 1, and Paris-Dauphine during this research. The research is supported in part by National Science Foundation under Grant No.1501031, Wisconsin Alumni Research Foundation, and the Herchel Smith foundation of the University of Cambridge. 


\section{REFERENCES}

[1] Arsenio, D.; Saint-Raymond, L.: From the Vlasov-Maxwell-Boltzmann system to incompressible viscous electro-magnetohydrodynamics, arXiv:1604.01547

[2] Cao, Y.: Regularity of Boltzmann equation with external fields in convex domains of diffuse reflection, submitted, arXiv: 1812.09388

[3] Cao, Y.: A note on two species collisional plasma in bounded domains, preprint

[4] Cercignani, C.; Illner, R.,; Pulvirenti, M.: The mathematical theory of dilute gases. Applied Mathematical Sciences, 106. Springer-Verlag, New York, 1994.

[5] Chen, H.; Kim, C.; Li, Q.: Local Well-posedness of Vlasov-Possion-Boltzmann system with Generalized Diffuse Boundary Condition, preprint

[6] Desvillettes, L.; Dolbeault, J.: On long time asymptotics of the Vlasov-Poisson-Boltzmann equation. Comm. Partial Differential Equations 16 (1991), no. 2-3, 451-489.

[7] DiPerna R.J.; Lions P.L.: On the Cauchy problem for Boltzmann equation: global existence and weak stability. Ann. Math. 130, (1989) 321-366

[8] Esposito, R.; Guo, Y.; Kim, C.,; Marra, R.: Stationary solutions to the Boltzmann equation in the Hydrodynamic limit. Ann. PDE 4 (2018), no. 1, Art. 1, 119 pp.

[9] Esposito, R.; Guo, Y.; Kim, C.; Marra, R.: Non-Isothermal Boundary in the Boltzmann Theory and Fourier Law. Comm. Math. Phys. 323 (2003), no. 1, 177-239.

[10] Devillettes, L.; Villani, C.: On the trend to global equilibrium for spatially inhomogeneous kinetic systems: the Boltzmann equation. Invent. Math. 159 (2005), no. 2, 245-316.

[11] Gilbarg, D.; Trudinger, N.: Elliptic partial differential equations of second order, Reprint of the 1998 edition, Classics in Mathematics, Springer-Verlag, Berlin, 2001.

[12] Gualdani, M.P.; Mischler, S.; Mouhot, C.: Factorization for non-symmetric operators and exponential H-theorem. Mem. Soc. Math. Fr. (N.S.) No. 153 (2017), 137 pp.

[13] Guo, Y.: The Vlasov-Poisson-Boltzmann system near Maxwellians. Comm. Pure Appl. Math. 55 (2002), no. 9, $1104-1135$.

[14] Guo, Y.: The Vlasov-Maxwell-Boltzmann system near Maxwellians. Invent. Math. September 2003, Volume 153, Issue 3, 593-630

[15] Guo, Y.; Kim, C.; Tonon, D.; Trescases, A.: Regularity of the Boltzmann Equation in Convex Domains. Invent. Math. January 2017, Volume 207, Issue 1, 115-290

[16] Guo, Y.; Kim, C.; Tonon, D.; Trescases, A.: BV-Regularity of the Boltzmann Equation in Non-convex Domains. Arch. Rational Mech. Anal. 220 (2016), no. 3, 1045-1093.

[17] Guo, Y.: Decay and Continuity of Boltzmann Equation in Bounded Domains. Arch. Rational Mech. Anal. 197 (2010), no. 3, 713-809.

[18] Guo, Y.; Jang, J.: Global Hilbert Expansion for the Vlasov-Poisson-Boltzmann System, Comm. Math. Phys. Volume 299, Issue 2 (2010), 469-501

[19] Guo, Y.: Regularity of the Vlasov equations in a half space. Indiana. Math. J. 43, 255-320 (1994)

[20] Glassey, R.: The Cauchy Problems in Kinetic Theory. Philadelphia: SIAM, 1996

[21] Glassey, R.; Strauss W.: Decay of the linearized Boltzmann-Vlasov system. Transport Theor Stat,Volume 28, Issue 2 (1999), $135-156$

[22] Glassey, R.; Strauss W.: Decay of the linearized Boltzmann-Vlasov system. Transport Theor Stat,Volume 28, Issue 2 (1999), $135-156$

[23] Hwang H.-J.; Velazquez J.: Global existence for the Vlasov-Poisson system in bounded domains. Arch. Rat. Mech. Anal. 195(3), 763-796 (2010)

[24] Jang J.; Masmoudi N.: Derivation of Ohm's law from the kinetic equations. SIAM J. Math. Anal. 44 (2012), no. 5, 3649-3669

[25] Kim, C.: Formation and propagation of discontinuity for Boltzmann equation in non-convex domains. Comm. Math. Phys. 308 (2011), no. 3, 641-701.

[26] Kim, C.: Boltzmann equation with a large external field. Comm. PDE. 39 (2014), no. 8, 1393-1423.

[27] Kim, C. and Lee, D.: The Boltzmann equation with specular boundary condition in convex domains. Comm. Pure Appl. Math. 71 (2018), no. 3, 411-504.

[28] Kim, C. and Lee, D.: Decay of the Boltzmann equation with the specular boundary condition in non-convex cylindrical domains. Arch. Ration. Mech. Anal. 230 (2018), no. 1, 49-123.

[29] Kim, C. and Lee, D.: Hölder Regularity Propagation for the Boltzmann equation Past an Obstacle, in preparation

[30] Kim, C.; Yun, S.: The boltzmann equation near a rotational local maxwellian. SIAM J. Math. Anal. 44 (2012), no. 4, $2560-2598$.

[31] Majda, A.J.; Bertozzi, A.L.: Vorticity and Incompressible Flow, Cambridge University Press, Cambridge, 2001.

[32] Maxwell, J. C.: On the Dynamical Theory of gases, Phil. Trans. Roy. Soc. London, 157, 49-88, (1866).

[33] Mischler, S.: On the initial boundary value problem for the Vlasov-Poisson-Boltzmann system. Commun. Math. Phys. 210, 447-466 (2000)

[34] Saint-Raymond, L.: Hydrodynamic Limits of the Boltzmann Equation. Springer, Berlin, Heidelberg, 2009.

[35] Strain, R.: Asymptotic Stability of the Relativistic Boltzmann Equation for the Soft Potentials. Comm. Math. Phys. 300 (2010), no. 2, 529-597.

E-mail address: chanwoo.kim@wisc.edu, ckim.pde@gmail.com

Department of Mathematics, University of Wisconsin, Madison, Wi 53706 USA 